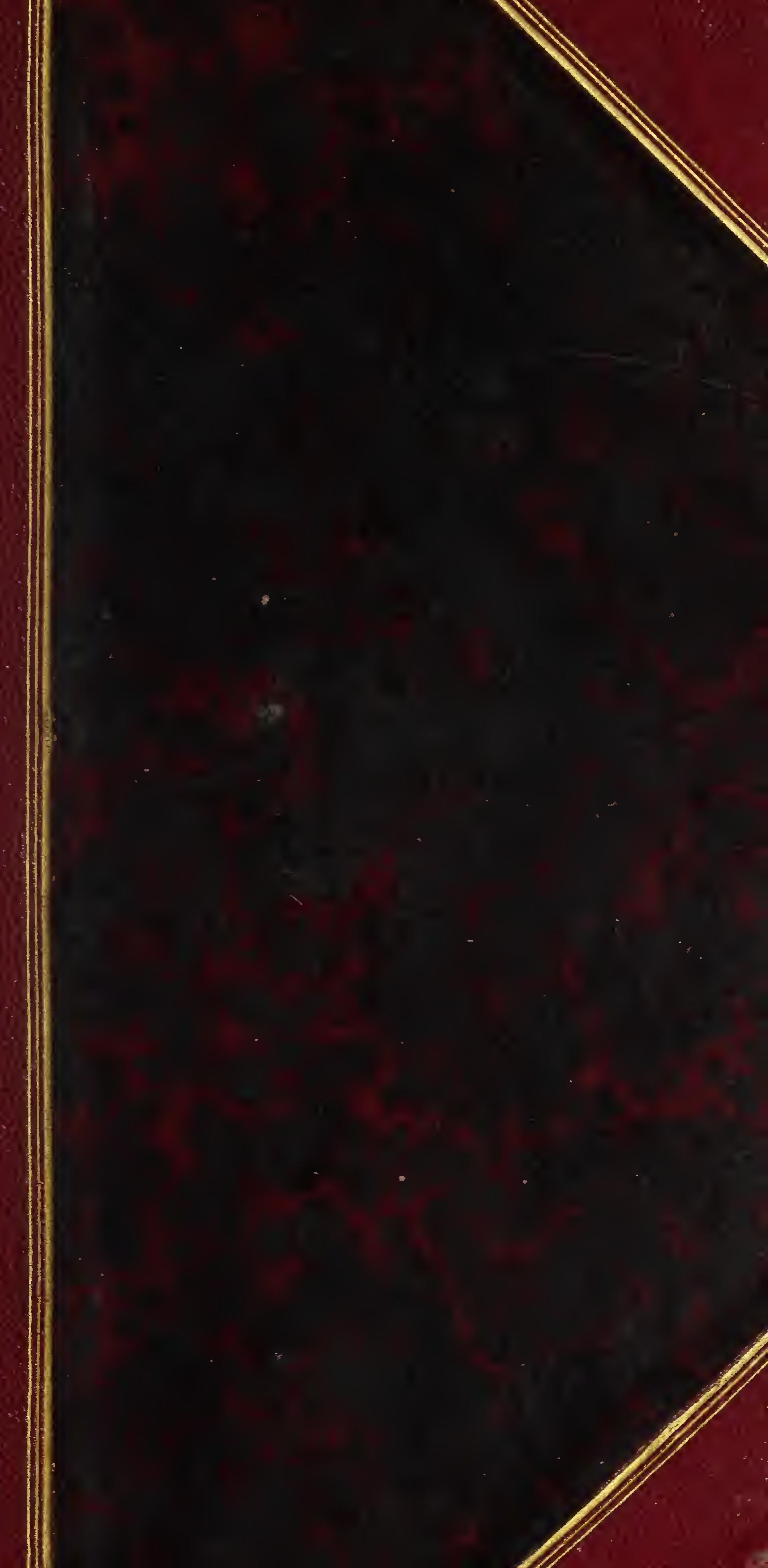


(3)

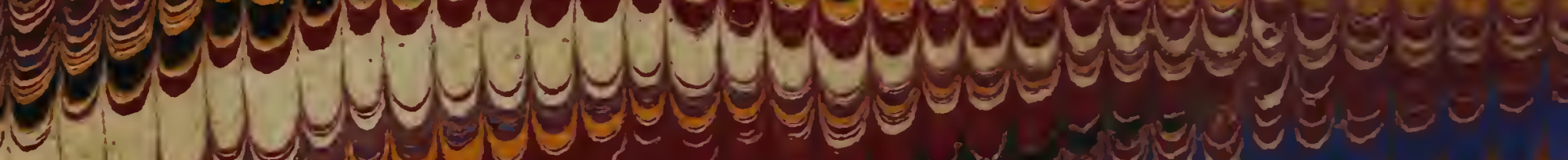
1.

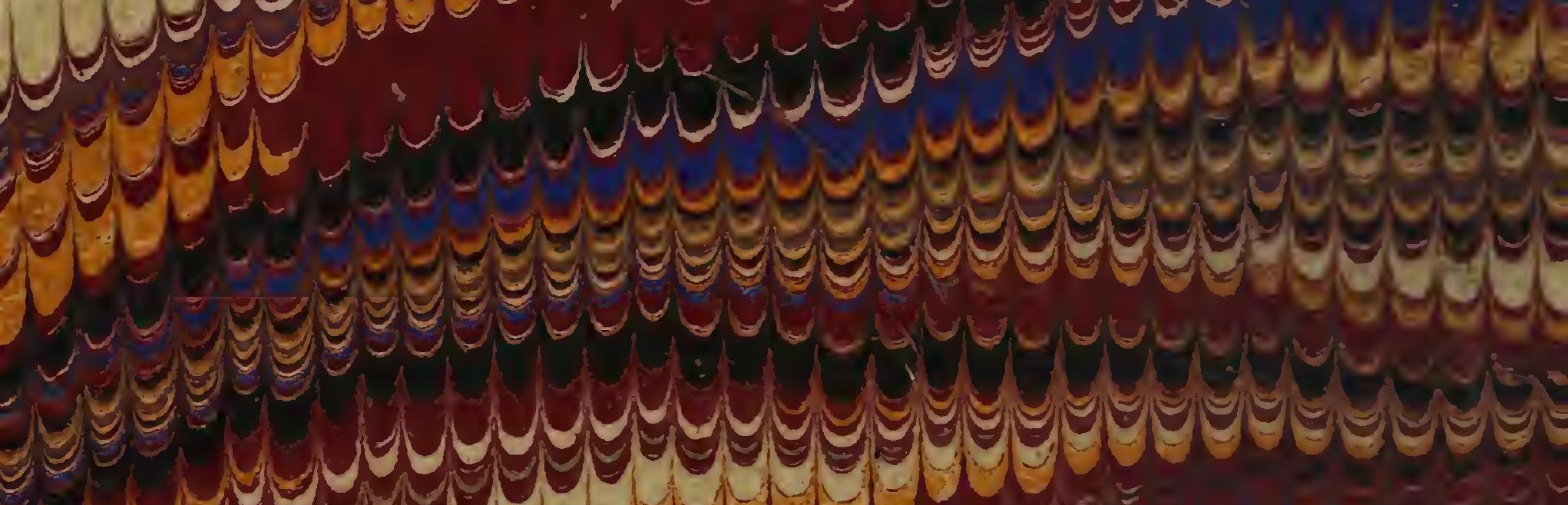

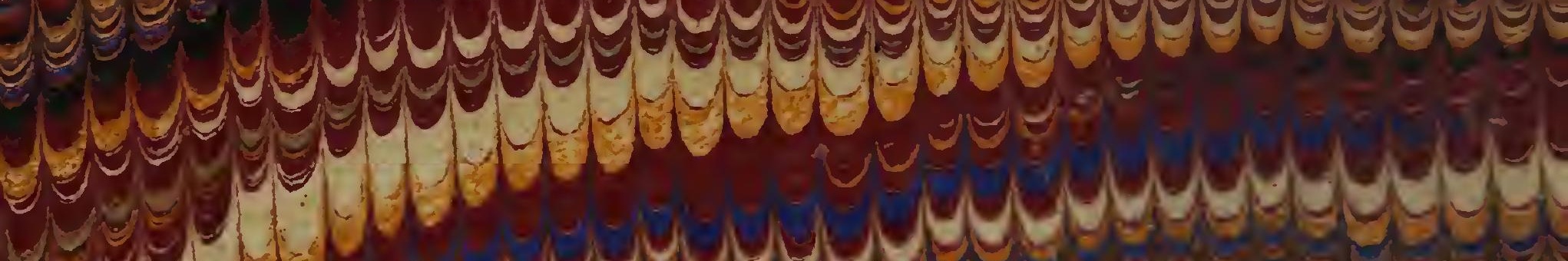
Co

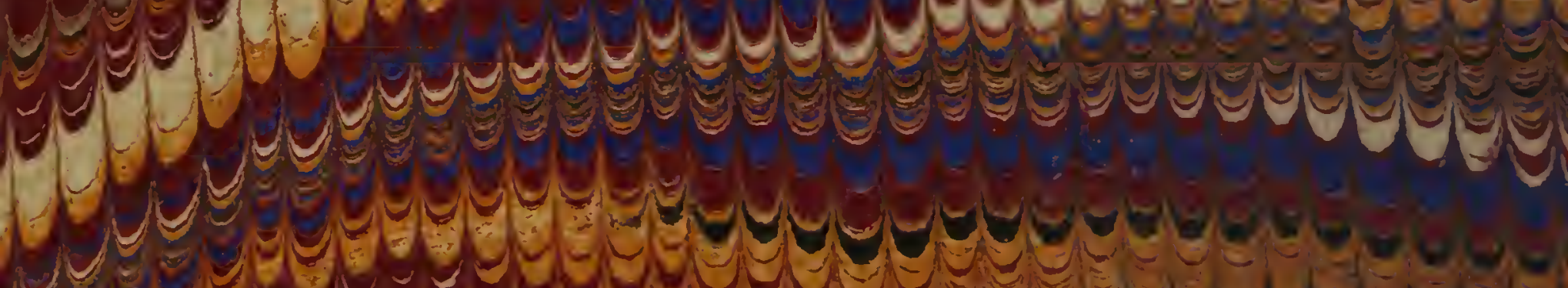
Y 930 1990

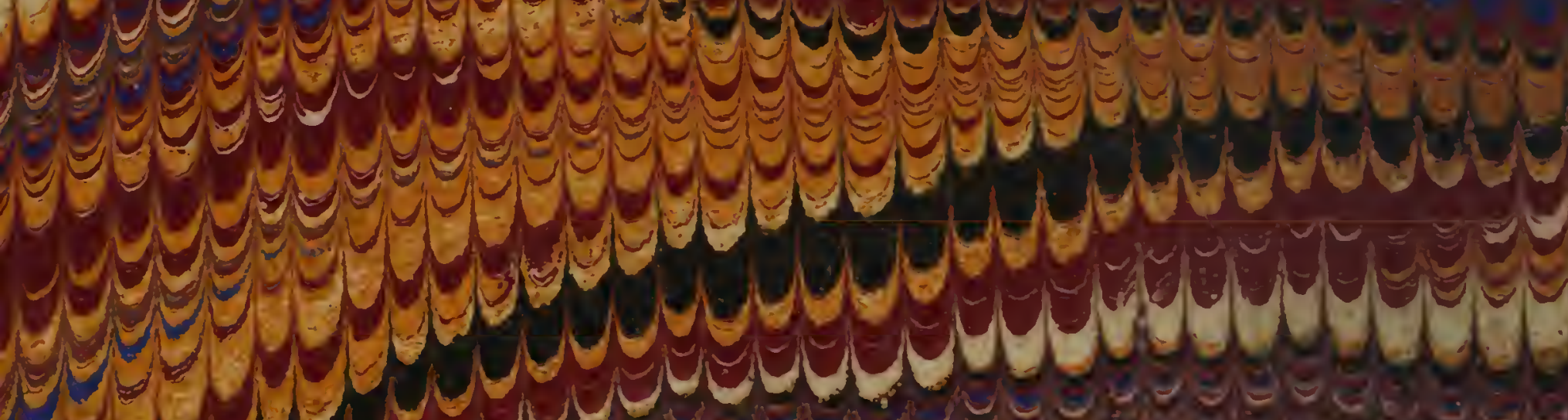

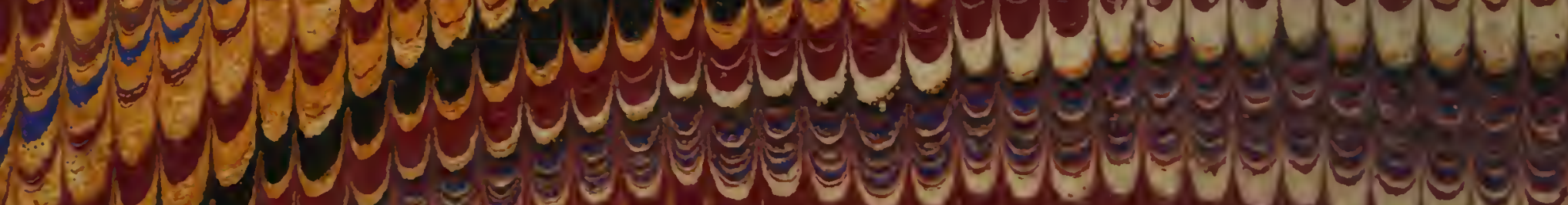

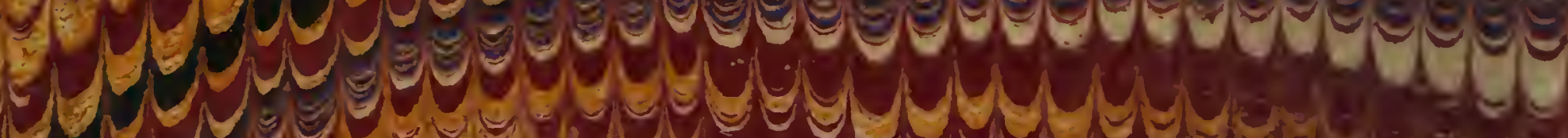

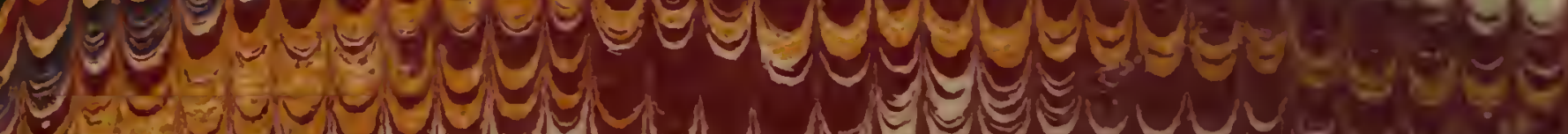

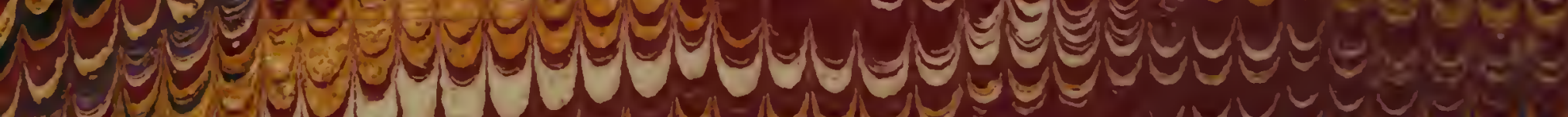
Q

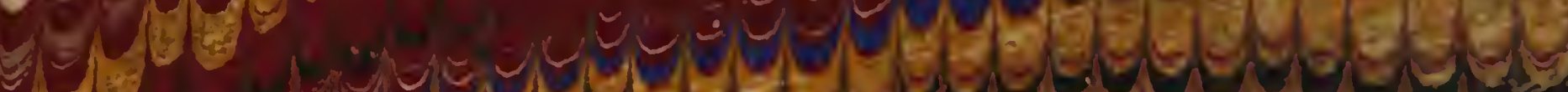

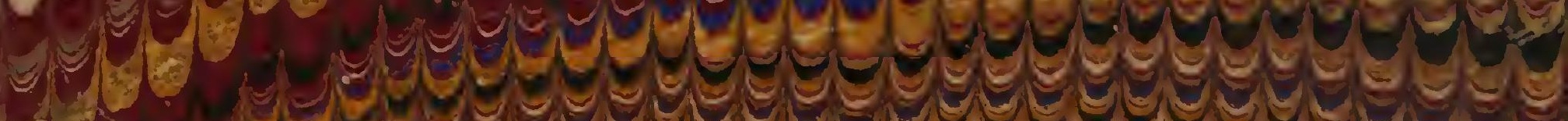

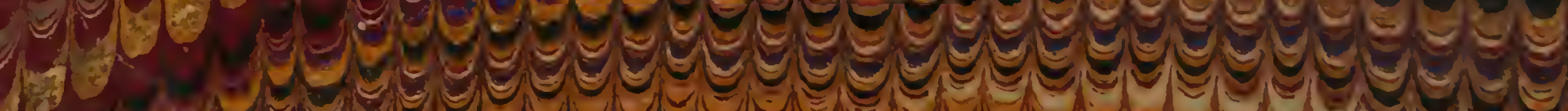




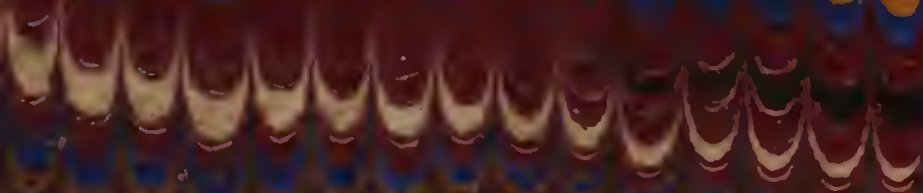

arb

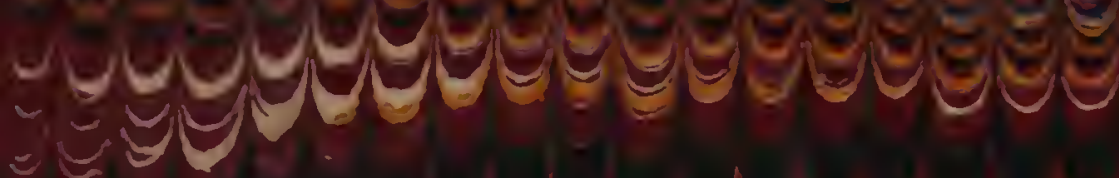

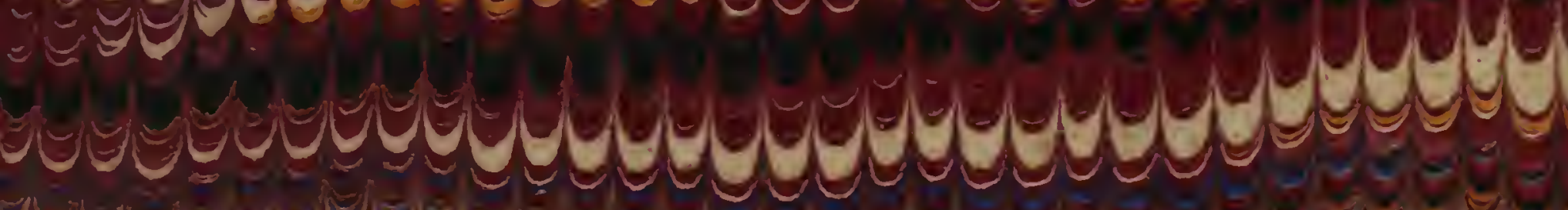
ur boy Haw 4 T J 00100 C To y z s

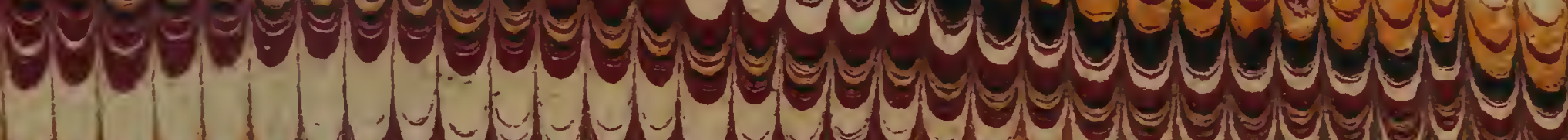

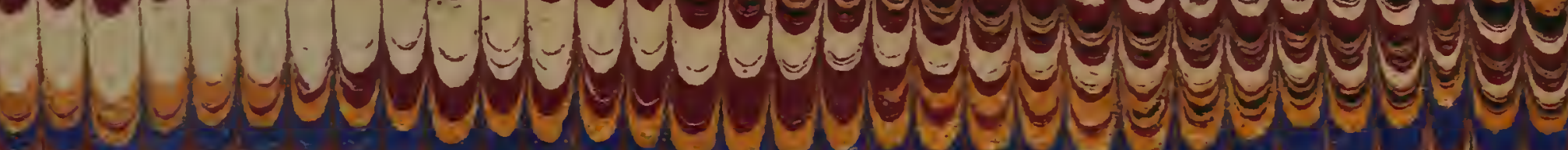
作 S 政 ach toun ,

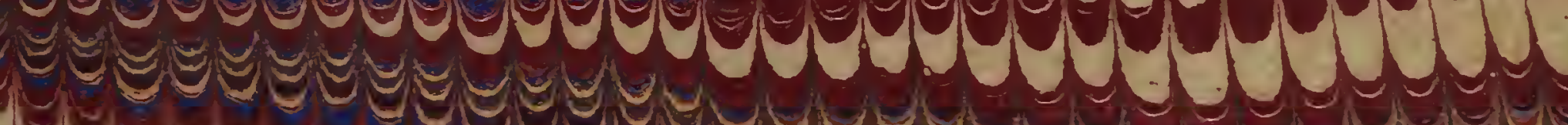

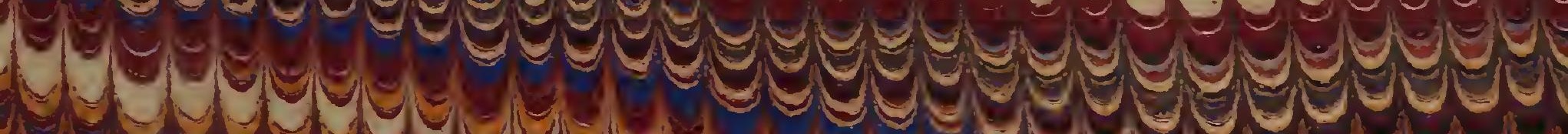

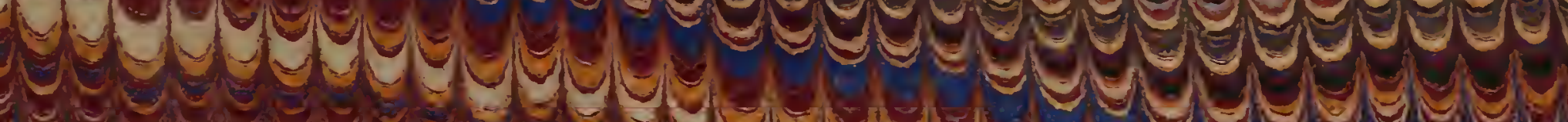
C

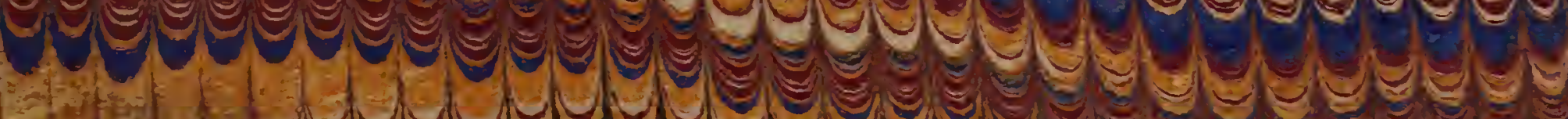

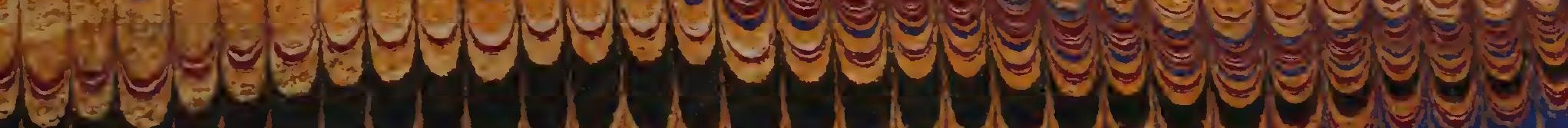
y you w y y y y y y -

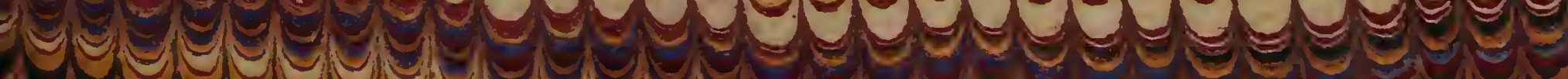




$$
\begin{gathered}
5.8 \\
4.0 \\
7 .
\end{gathered}
$$


L.ztenisherbon

bonpos at the sake of

$$
\text { Juppoute Crosse }
$$

hor. 21. 1899 



\section{MUSEUM BOLTENIANUM.}

\section{Yxicitants doc}

von dem verstorbenen

\section{Herrn Joachim Friedrich Bolten
M. D. und Physicus in Hamburg hinterlassenen}

bortefflichen sammlung

\section{Conchylien, Mineralien} u $\mathrm{d}$.

\section{Kunstsachen}

di e

am 26. A pril d. J., Morgens um $10 \mathrm{Uhr}$ öffentlich verkauft werden sollen

durch den Makler

\section{$\begin{array}{llllllll} & \boldsymbol{o} & \boldsymbol{h}^{s} & \boldsymbol{N} & \boldsymbol{o} & \boldsymbol{o} & \boldsymbol{d} & \boldsymbol{t}\end{array}$}

Hïxter No. 69.

Cat. XXXIII.

Mit vier auf Stein gezeichneten Platten seltener Conchylien Hamburgischen Steindrucks.

H amburg 1819.

Gedruckt bei Conrud MYiller, Bohnenstrasse No. 151. 


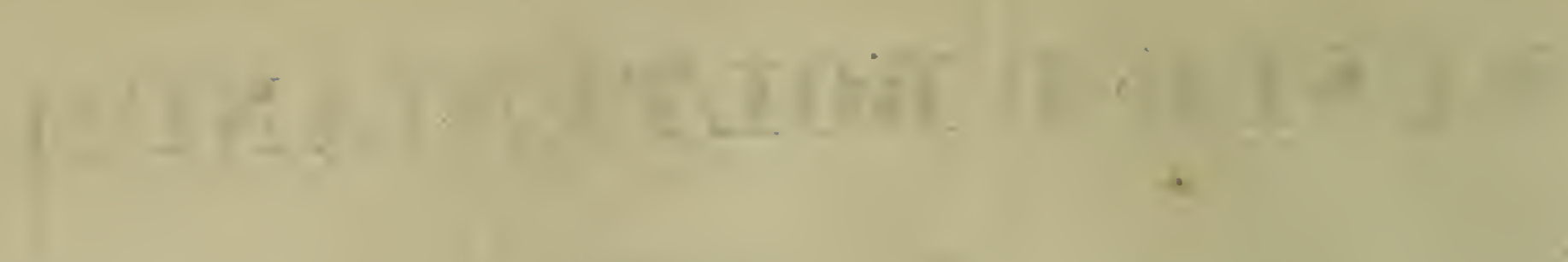

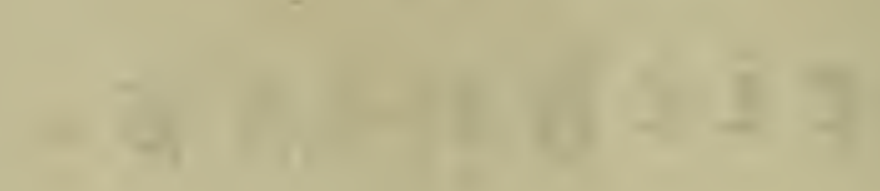

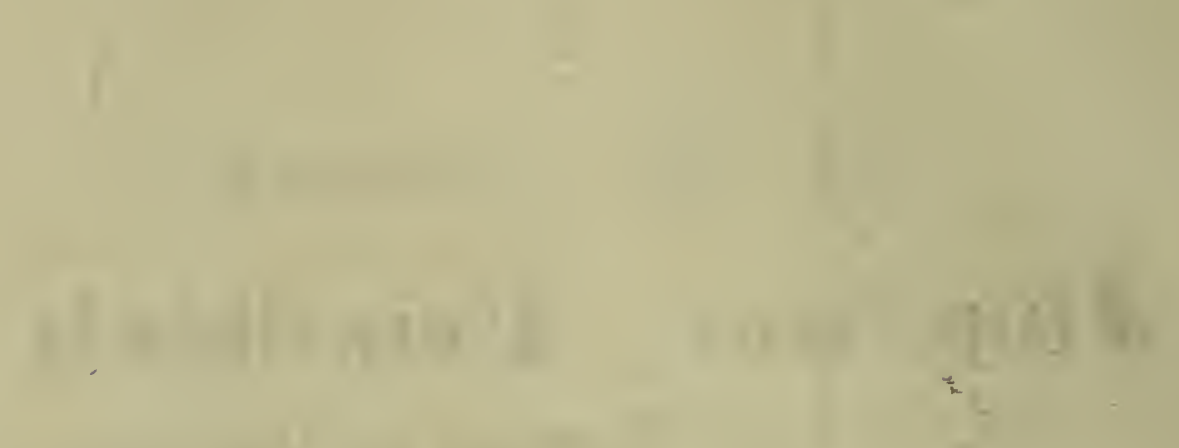

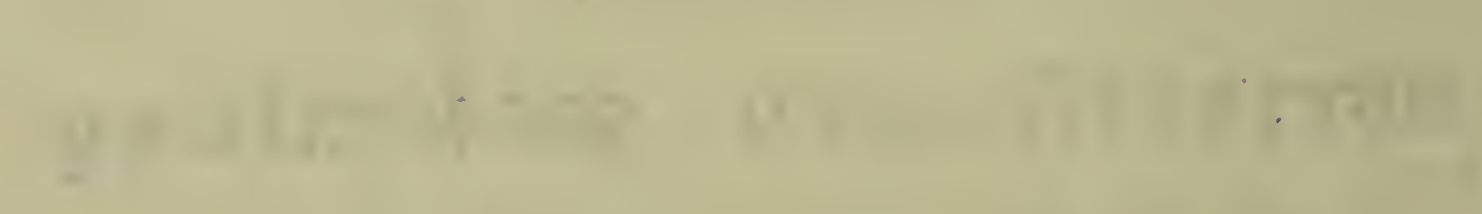

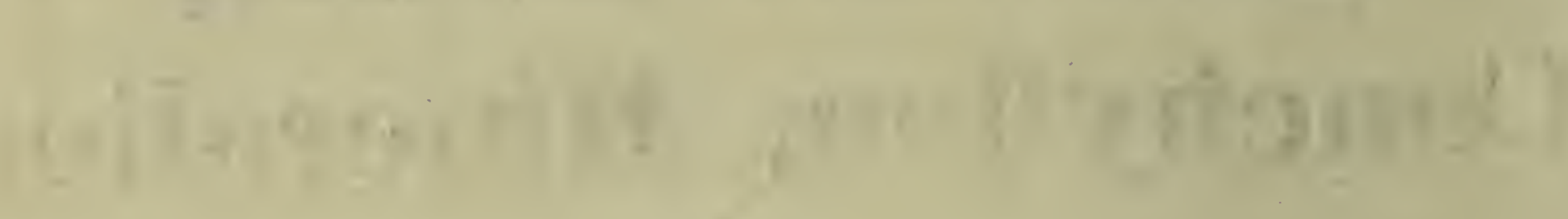
, heu

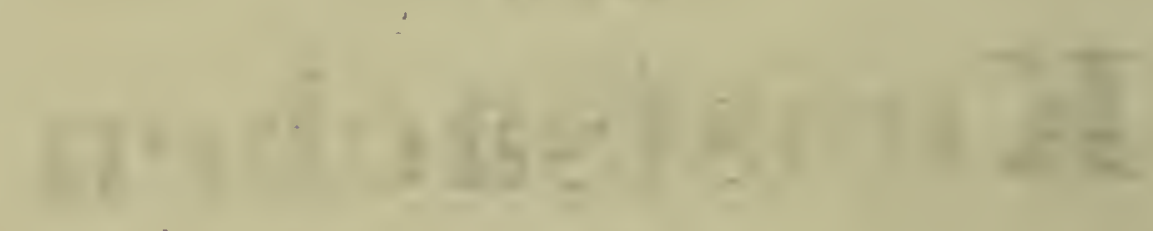

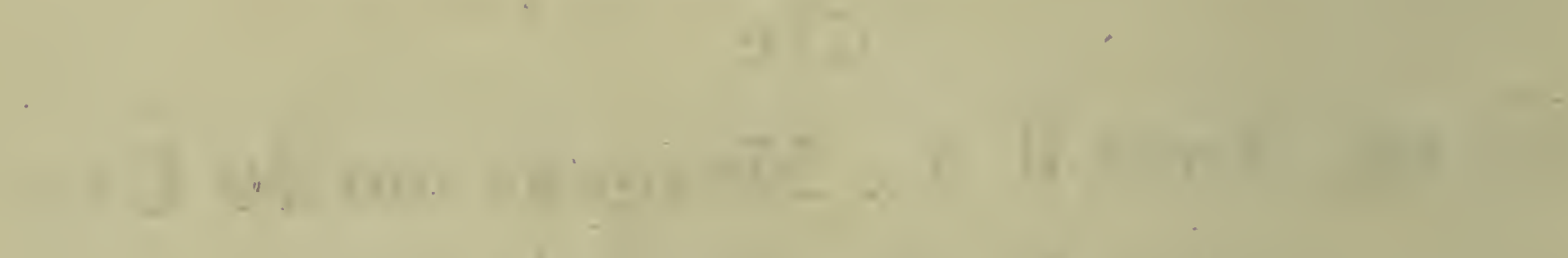
1 in $^{-1}+2+1=+1$ 
HAHBURG, Jamiar 1819.

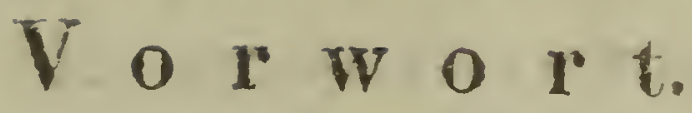

Freunden des Wissens wird der Nahme des Herm Doctors Bolten ein bleibendes Denkmal seyn. Mit Nennung desselben. wird sich, seinen Bekannten, die Rückerinnerung des Genusses erneueren, die ein stets bereitwilliges Zeigen der treflichen Kunst- und NaturSchätze Ihnen schaffte, die durch Kenntnisse ausgebreitete Bekanntschaft und Aufopferung der Besityer durch 40jährige Auswahl zusammen zu bringen das Glück hatte. Denen, die den achtbaren Verstorbenen nicht persönlich kannten, wird es angenehm seyn, diese vorziigliche Sammlung jetzt sehen, und durch Ibnen angebotenen Verkauf dersclben, sich in Besitz von werthvollen $\mathrm{Sa}$ chen, die selten vorkommen, setzen zu können.

Nur kann ich das allbekannte verdiente Lob dieser Sammlung wiederholen, die wohl unbezweifelt - zumal was den Conchyologischen Theil betrifft - durch Schönheit und Seltenheit der darin enthaltenen Exemplare, zu den ersten Sammlungen zu zählen. Was dez Liebhaber zu finden, sagt nachfolgender Catalog, der im Jahre 1798 schon von Herrn P. F. Röding nach Gmelins 13ter Ausgabe des Linnéischen Systems mit vieler Surgfalt aus bemerkten Schriftstellern zusammengetragen und so gut wie möglich, mit Nachwcisungen derselben versehen ward. Da die Sammlung manches sehr Seltene, wenig Bekanute, noch nicht Beschriebene cnthielt, habe ich in der neuen, von Druckfehlern möglichst freyen, mit der Sammlung von mir nochmals verglichenen Auflage des gegenwärtigen Catalogss einige Zeichnun- 
gen dieser, im Steindruck beygefügt, die ich Liebhabern zur nachsichtlichen Aufnahme zu empfehlen wage.

Der verstorbene Herr Mag. und Abt A. A. H. Lichtenstein hatte die Güte, dem ersten Katalog eine lateinische Vorrede vorzusetzen, die, weil dadurch das Andenken zweyer schätzbarer Männer geehrt wird, wegzulassen ein Vergehen gewesen seyn würde.

Ein im Catalog den Nummern vorgedruckter Stern, gibt dem Leser Gelegenheit zu leichterer Auffindung des Trefflichen oder vorzuiglich Seltenen. Der Werth der kostbaren Stücke der Sarnmlung wird noch durch treffliche Erhaltung erhöht; es darf der auswärtige Besteller versichert seyn, dass kein defectes, werthloses, von zweyschaaligten nicht passendes Stuick ihm zu Theil werden wird. Wo eine Beschädigung sich fand, habe ich sie zu bemerken gesucht.

Mit sorgsamer Genauigkeit und Gewissenhaftigkeit werde ich, die mir von fremden Sammlern, in portofreyen Briefen, gefälligst zu gebenden Aufträge besorgen. Ich wage mich dazu zu empfehlen und darf fest versichern, dass die erstandenen Exemplare Ihnen unvertauscht zukommen werden. Um eine genaue Bemerkung der Catalogs - Paginas und der Doppelt - Nummer bey den Aufträgen, bitte ich, um Versehen zu vermeiden.

Der Verkauf geschieht gegen baare Bezahlung in hiesig grob Courantem Gelde bey Lieferung. Auswärtige wollen indess nur gefälligst Thre Aufträge in der Landesmünze geben, ich werde sie nach unserm CourantGelde genau berechnen.

Indem ich dicse treffliche Sammlung, deren Theilung sehr zu bedauren, nochmals bestens empfehle, erbiete ich mich zugleich zu schriftlicher und mündlicher noch näheren Auskunft auf zu machende Anfragen.

Johannes Noodt. 


\section{B. S.}

Praeter innumeros alios thesauros rerum naturalium, quas Vir Experientissimus atque doctissimus Boltenius, Medic. Doct. et Poliater primarius, de republica litteraria pariter atque civili universa, in primis vero de Hamburgensi aeternum promeritus, per quadraginta annos collegerat, immensam reliquit conchyliorum, magna ex parte rarissimorum eorumque splendidissimorum et optime conservatorum supellectilem.

Cuius indicem systematicum ab ipso beato possessore quam diligentissime curatissimeque elaboratum edituri heredes id mihi negotii dederunt, ut a Viro universae historiae naturalis praesertim vero conchylio= logiae amantissimo, peritissimoque $\boldsymbol{P}$ etro Friederico $\boldsymbol{R} \ddot{o}$ ding magna sollertia revisum et synonymia locupletiore adauctum, brevi praefatione Latina instruerem. Quod quidem, utpote innumeris benevolentiae monumentis ab ornatissima familia Bolteniana devinctus, licet minime pro conchyliologo me venditare ausim, attamen 
recusare nolui. Offendentur enim forsan primo obtutu conchyliologiae arbitri pariter atque amantes tot novis inauditisque nominibus, praesertim genericis in hoc catalogo obviis. Qua de re paucis monendi sunt, haec singularis publicaque auctoritate carens nomenclatura unde originem traxerit. Elaboraverat nempe Praeclarissimus Boltenius novum et proprium Systema naturale conchylyologiae, ab omnibus aliis priorum auctorum systematibus multune diversum, idque adeo subtiliter adornatum in artis certae sive disciplinae formam, secundum peculiaris philosophiae conchyliologicae regulas exaedificatam constructanque, redegerat, ut olim edita hac methodo sua naturali studendi historiae conchyliorum, propemodum instar dictatoris vel alterius Linnaei in hac parte naturae regnaturus videretur. Prodierunt re ipsa loco speciminis aliquot plagulae a Viro experientissimo doctissimoque Dominico Schultze, M. D. acuto naturae verique indagatore, sub ipsis beati Boltenii auspiciis adumbratae. Sed ipsum opus integrum proh dolor! acerba Schulaii morte interruptum, quum laboribus occupata distractaque senectus Boltenio impedimento esset, quo minus solus limaret cumque orbe litterato communicaret, sollertem atque incredibili sagacitate elucubratam methoduin conchyliologicam. Cuius quidem fundamentum solidum nitebatur superstructum ideae sim- 
plici, eique egregiae; naturam nempe ossea testaccorum tegumenta varia condituram initium fecisse a simplici et plano tegmine atque inde magis magisque torto volumine ad reliquas sensim elegantiores intricatioresque formas univalvium, bivalvium et multivalvium conchyliorum perexisse. Ingeniosum sane, auctoreque tum naturae ipsius tum systematis condignum commentum! Utinam beatus Boltenius reliquisset aliquam ordinum et generum naturalium, in quos testacea ex hoc principio distinguendi dilaberentur sciagraphiam, quam praefationi nostrae inserere potuissemus in magnum usum eorum, qui catalogum sequentem cum intelligentia perlegere dignati forent. Nunc vero proh dolor nil nisi nova testaceorum gencra, nisi omnia, plurima certe ex profunda solidaque scientia conchyliologica constituta, tamquam disiecti membra systematici apparent. In quo, etsi lector, in deliciis modo habens testas ob formarum et colorum varietatem, hallucinari facile poterit, praesertim ubi a synonymia desertus, erat circa species determinandas, multum tamen proficiet gnarus et vere systematicus arbiter, quando Boltenii genera cum Linnaei aliorumque auctorum classificationibus comparabit. Nihil certe magis optandum foret, nisi, ut integer hic testaceorum thesaurus neutiquam auctione distractus, in manus talis viri veniret, qui incredibiles labores quos Boltenius noster in comparando et adornando lioc immenso museo 
conchyliologico exantlavit, in honorem summi numinis, et emolumentum historiae naturalis convertere possit, ac velit. Cetera, lectori, conchyliorum amanti scitu necessaria, patebunt ex insequenti praefatione vernacula.

Dabam Hamburgi d. X. mens. Septembr. 1798.

M. Ant. Aug. Henr. Lichtenstein. 


\section{Lade 1. \\ Patella. Schüssel-Muschel.}

1. Perforato. Durchbohrte.

* Foramine centrali. Die Oefnung in Mitielpunkt.

1. Sulcatce. Gefurchte.

1...1 P. Mitella. Die Haube. Gmel. P. nimbosa. sp. 197. Mart. 1 Theil fig. 91 \& 92.1 Stück.

$2 . . . \quad *-2$ st.

3...2 P. Grisea. Die aschgraue Patelle. Gm. P. Caffra; sp. 201. Mart. 1 Th. tab. 11. fig. 95. 1 St.

4...3 P. Infundibulum. Der Trichter. Gm. sp.65. P. infundibulum; Schröt no Iitt. 3 p. 72 n. 135. 1 St.

5...4 P. Umbrosa. Die dunkle Patella, da Costa. tab. 7. fig. A. 1 St.

6...5 P. Nimbosa. Die schwarze Schein-Patelle. Gm. sp. 196 P. nimbosa. Knorr Vergn. d. Augen. IV. tab. 29 fig. A. Mart. I. tab. 11 fig. 90. S St.

7,6 P. Radiata. Die Strahl-Patelle. Gm. sp. 14t Patella radiata. Ch. C. 10 p. 329 t. 168 fig. 1618. 1 St.

\section{Costatce. Geribte.}

8...7 P. Limbata. Die gesäunte Patelle. Pat. porphyrezonias, sp. 203. Mart. 1 Th. tab. 12 fig. 102, 3, 4. 17 St.

9...8 P. Rubeola. Die röthliche Patelle. Patella rosea, sp. 204. Mart. 1 tab. 12 fig. 105. 4 St.

$10 \ldots 9$ P. Variegata. Die gerippte Patelle. 1 St.

11...10 P. Viridana. Die grüne Patelle. 3 St.

12...11 P. Viridis. Die grünliche Patelle. 1 St.

3. Clathraíce. Gegitterte.

13...12 P. Rudis. Die plumpe Patelle. Gm. Pat. jamaicensis, sp. 200. Mart. 1 tab. 11 fig. 94. Lister Conch, 1 tab. 528 fig. 6. 3 St.

14...15 P. Cancellata. Die gegitterte Patelle. Gm. P. graeca, sp. 195. Mart. 1. tab. 11 fig. 98. 2 S..

$15 \ldots \approx-3$ st.

$16 . . \beta-2 S_{t_{1}}$ 
17...14 P. Cingulata. Die gegürtelte Patelte. Gm. 190. Gm. Pat. Scapha, sp. 191. Naturforscher 18 p. 10 t. 2 f. 8.

4. Levigule. Glatte.

18...15 P. Ferula. Die Schiene. da Costa t. 7 fig. 7. 2 St. 19...15 * P. Purulenta. Die bestaubte Patelle. 2 St.

\section{Patella. Schüssel-Muschel.}

1. Perforalce. Durchbohrte.

** Foramine excentrico. Die Oefnung seitwirts.

1. Elevata. Erhöhte.:

20...16 P. Clavus. Das Hünerauge. 2 St.

21...17 P. Lacrymalis. Die Thränen-Patelle. 1 St.

22...18 P. Graeca. Die griechische Patelle. Gmel. sp. 195. Mart. 1 tab. 11 fig. 98, 101, 104. Fnorr Vergn. 1 tab. 30 fig. 3. 3 St.

\section{Depressa. Gedrückle.}

23...19 P. Nummularis. Die Groschen - oder weisse Schlïisselloch-Patelle. Gm. Pat. Pustula, sp. 194. Chemn. 10 t. 168 f: $1632 ; 33.3 \mathrm{St}$.

24...20 P. Craniolaris. Die rothe Schlüsselloch-Patelle. Gm. P. pustula, sp. 194. $\beta$ Mart. Beschr. Berl. Naturf. 2 t. 12 fig. 4, 5. Patella unguis. Petiv. Gaz. tab. 3 fig. 12. 1 St.

25..21 P. Pustula. Die Bंlatter. Gm. sp. 191. Ch. C. 10 p. 338 tab. 168 fig. 1632, 33. Schrüt. Einl. in die Conch. 2 t. 5 fig. 8. Iister 523 fig. 3. 1 St.

\section{Patella. Schüssel-Muschel.}

2. Tmperforatoe. Undurchbohrte.

* Míargine edentulo. Mit ungezähnten Rand.

1. Vertice rostrato. Mit geschnörkeltem Wirbel. ๙. Uncinatac. Hakenfürmige.

26...22 P. Hungarica. Die Zoots-Kappe. Gm. sp. 89. Pat, ung arica. Lister Conch. tab. 544 f. 32 . Knorr Vergn. 6 tab. 16 fig. 3. Mart. 1 tab. 12 fig. 107, 108. 6 St., wovon 2 St. mit der Schleimhaut.

27...25 P. Pannonica. Die Narren.Kappe. Lister tab. 541 fig. 37. 2 St. 
28...25* P. Fissura. Die eingeschnittene Patelle. Gm. 192. Lister C. t. 543 f. 28 . Mart. 1 t. 12 f. 109, 110. 1 St. 29...23** P. Tricarinata. Die Grenadicr-Mütze. Gm. sp. 92. Schröt. Einl. in die Conch. t. 5 fig. 2. da Costa tab. 4 fig. 9. 4 St.

30...24 P. Cornu Copiae. Das Füllhorn, da Costa tab. 5 fig. 7. 4 St.

31...25 P. Rugosa. Die runzliche Patelle. Gm. Pat. mittula, sp. 82. Lister t. 544 f. 31. Níart. 1 tab. 12 fig. 111 , 112. 2 St.

92..26 P. Lactea, Die Nachtiappe. Gm. sp. 224. Pat. Iactea. Schröt. n. Litter. 3 p. 152 n. 294. Lisier tab. 544 fig. 33. Favanne tab. 4 fig. B. 1 St.

\section{ß. Labiatae. Gezüngelte.}

33...27 P. Equestris. Die Fischweiber-Haube. Gm. sp. 1. P. equestris. d'Augenv. Conch. tab. 2 f. Th. Cabochon. Mart. 1 tab. 13 fig. 117, 118.1 St.

34...28 P. Undulata. Die Matrosen-Mintze. P. equestris $\mathrm{Gm}$. sp. 1 (mit'stumpfer Spitze) Mart. 1 tab. 13 fig. 119, 120. 3 St.

35...29 P. Crenulata. Die Glocke. Gialthi. t. 9 f. X. 2 St. 36...30 P. Contorta. Die Zickzacli-Patelle. Gm. P. contorta, sp. 11. Naturf. 9 p. 199 t. 3 f. 3, 4. Külmmerer Mu. seum P. Rudolst. p. 23 tab. 2 fig. 8. 2 St.

37...31 P. Spiralis. Die Schnecken-Patelle. Gm. Pat. contorta, sp. 11. da Costa t. 6 f. 2. 1 St.

38...32 P. Dispar. Die gezüngelte Tappe. Gm, P. contorta, sp. 11. INaturs. 18 St. tab. 2 fig. 13. St.

39...33 P. Turcicu. Der Turban. Gm. Pat. sinensis, sp. 3. Mart. 1 tab. 13 fig. 123, 124. 3 St.

40...34 P. Electrica. Die Bernstein-Patelle. Gm, Pat. sinensis, sp. 3. 2 St.

41...35 P. Tectun-Chinense. Das chinesische Dach. Grn. sp. 1. Equestris. Knom Vergn. 6 t. 35 f. 4. Mart. 1 tab. 13 fig. 125, 126. Cliemn. 10 t. 168, 1630, 31: 2 St.

42...36 P. Chinensis. Der Regen-Sclirm. Gm. Pat. sinensis, sp. 3. Argenv. tab. 2 fig. F. Mart. 1 tab. 13 fig. 121, 122. A St.

43...37 P. Mammnla. Die Brust-Warze. Gm.P.mammillaris, sp. 91. Lister t. 537 f. 17 . Favanne t. 6 f. $63.8 \mathrm{St}$. 
44...38 P. Plicata. Der Kragen. Gm. sp. 13. Patella plicata. Natuj'f. 18 t. 2 f. 12,12 a. Fav. t. 4 f. CC. 2, 2.1 St. *45...39! P. Hispida. Dic Stachel-Patelle. Gm. P. aculeata, sp. 6. Favanne tab. 4 fig. B. 3. 1 St.

$\dot{\gamma}$ Neritoideae. Kammerförmige.

45.०440 P. Fornicata. Die Pantoffel-Patelle. Gm. Pat. For. nicata, sp. 5. Knorr 6 t. 21 f. 3. Mart. 1 tab. 13 fig. $129,130.5$ St.

47...41 P. Porcellana. Die Porcellan-Patelle. Gm. Pat. porcellana, sp. 4. Mart. 1 tab. 13 fig. 127, 128. Ch. C. 9 tab. $124 \mathrm{f} .1032 .5 \mathrm{St}$.

48...42 P. Nucleus. Dit Kern-Patelle. Gm. Pat. porcellana, sp. 4. Favanne tab. 4 fig. E. 2. 3 St.

49...43 P. Crepida. Die durchsichtige Patelle. Gm. sp. 17. Crepidula. Adans. Seneg. 1 tab. 2 fig. 9. Garnot. Faa vanne tab. 4 fig. G. $22.1 \mathrm{St}$.

*50...44! P". Cymbeola. Das Kähnchen. 1 St.

*51...45! P. Appendiculata. Die Zunge. 1 St.

${ }^{*} 52 . .46$ ! P. Preticulata. Die Netz-Patelle. da Costa tab. 6 fig. 4. 2 St. (1 St. im Schleim.)

53..47 P. Haliotoidea. Die geschnörkelte Patelle. Gm. Pat. aculeata, sp. 6. Chemn. 10 tab. 168 fig. 1624, 25. da Costa tab. 6 fig. I. 1. 4 St.

No. 44,45 und 46 sind sehr seltene Stücke.

2. Vertice absoleto. Mit unkenntlichem Wirbel.

«. Ovato foliaceae. Eyrund blattförmig.

*54...47* ! P. Umbracula. Der Sonnen-Schirm. Gm, Pat. umbellata, sp. 146. Mart. 1 tab. 6 fig. 44 . Chemn。 10 tab. 169 fig. 1645, 46: 3 St. Sehr selten.

\section{Lade 2.}

B. Ovàto compressae. Eyrund, seitwärts gedrückte. 55...48 P. Compressa. Der Ellbogen. Gm. Pat. compressa, sp. 185. Knorr Vergn. 6 t. 28 f. 1. Lister t. 541 f. 25. Mart. 1 tab. 12 fig. 105. 5 St. (2 St. sind sehr gross.) 55...49 P. Lutea. Der gelbe Huth. v. Born. t. Is f.S. 2 St. $57 \ldots 50$ P. Stricta. Der braune IIuth. 1 St.

*58...51 ! P. Ulna. Die Wanne, viel ähnliches ron P. umbella, sp. 71, nur ist dieses Exemplar melır gewülbt wie die Ellbogen - Patelle. I St. Selir selten. 
*59...52 ! P. Gondola. Die Gondel. da Costa tab. 3 fig. 9. 1 St. Sehr selten.

60...53 P. Pectinata. Dic Kamm-Patelle. Gm.sp.93. Schröt. Einl. in d. Conch. tab. 5 fig. 3. Mãrt. I tab. 11 fig. 115, 116. 6 St. (2 St. sind etwas defect.)

$\%$ Ovato simplices. Eyrund einfache.

61...55 P. Smaragdina. Der Smaragd. da Costa tab. 4 fig. 44. 5 St.

62..55 P. Micans. Die Schimmer-Patelle. Schrüt. Einl. in die Conch. 2 tab. 5 fig. 13. 9 St.

63..56 P. Pellucida. Die durchsichtige Patelle. Gm. 139. Knorr V. 6 t. 28 f. 6. Ch. C. 10 t. 168 f. 1620, 21. v. Born tab. 18 fig. 9. 3 St.

64...57 P. Testudinaria. Die Schildkröten-Patelle. Gm. 134. Knorr V. 1 tab. 21 f. $1 \& 3$ t. 30 f. 2-5. Argenv. C. tab. 2 fig.P. Mart. 1 tab. 6 fig. $15-49.9$ St. (wovon 1 Stück etwas defect.)

65...58 P. Intertexta. Das Gervebe. Gm. 195. testudinalis. Ch. C. 10 t. 168 f. 1614, 15. Favanne t. 1 f. Q 2. 8St. 66.-.59 P. Diaphana. Die Warze. Lister t. 543 f. $26 \AA$. $2 \mathrm{St}$. *67..60 P. Nova Zeelandiae. Die Neu-Seelands-Patelle. 7 St. 63...61 P. Taitensis. Die Otalieite. Faranne t. 1 f.N. N. 3St. 69..62 P. Capillacea. Die Iraar - Patelle. Gm. 175. Mart. I tab. 7 fig. 58, 59. 4 St.

70..66 P. Digitale. Der Fingerliut. Gm. sp. 176 6. Mart.

1 tab. 7 fig. 57. 6 St.

71..64 P. Capillata. Gm. sp. 176. Mart. fig. 56, 5\% I St.

72..65 P. Papillaris. Die dicklinigte Patelle. Gm- P. leuco. pleuta, sp. 34. Lister t. 539 f. 22. Inorr 6 tab. 28 fig. 9. $2 S t$.

$79 . .66$ P. Mtorio. Der Mohr. 1 st.

$71 \ldots 67$ P. Africana. Die africanische Patelle. 2 st.

75..68 P. Nigrita. Der Neger. Favanne t. 1 f. B. 1. 1 St. 76..69 P. Bisurca. Die Gabel. 2 St. .

77...70 P. Fulgur. Die Blitz-Patelle. 1 St.

78...71 P. Fungoides. Der Pilz. Lister 599 f. 23. 2 St.

80...72 P. Aranea. Die Spinne. $2 \mathrm{St}$.

S1...75 P. Clypeus. Die Schild-Patelle. $\cong$ St.

82... 74 P. Crocea. Die Saffrangelbe Patelle. P. crocea, Gm.

sp. 114. Schröt. 12. Litterat. 3 p. 83 n. 150. 1 St. 
83...75 P. Conus. Der Kegel. Gm. P. vulgata, sp. 23. Mart. 1 tab. 5 f. 38. Knorr V. 6 t. 27 f. 8.7 St. 84...76 P. Cuprea. Die broncirte Patelle. Patella cuprea Gm. sp. 61. 1 St.

85...77 P. Cornea. Die Horn-Patelle. 3 St:

*86...79 !! P. Oculus Cati. Das Katzen-Auge. 1 St. Sehr selten und schön.

S\%...79 P. Patera. Die Schale. Gm. P. testudinaria, sp. 134. Mart. 1 tab. 6 fig. 4.5. 1 St.

*88...79* !! P. Aurora. Die Morgenröthe. 1 St. Sehr schön und selien.

*89..79** P. Glacialis. Die Spiegel-Patelle. 1 St.

90...80 P. Circumscripta. Die eingefasste Patelle. Gm. P. su. rinamensis, sp. 128. Mart. 1 tab. 7 fig. 50. 2 St.

91...81 P. Hidatis. Die Blase. Lister Conch. tab. 542 fig, 25. I St.

\section{Lade 3.}

\section{Patella. Schüssel-Muschel.}

2. Imperforatc. Undurchbohrte. ** IVargine undulato. Mit gewelltem Rand.

Simplices. Einfache.

92... \$2 P. Fiosea. Die rosenrothe P'atelle. Gm. Patella umbella, sp. 71. Mart. 1 t. 8 fig. 63.15 st.

צ̧...83 P. Rubicunda. Die rothstrahligte Patelle. Gm. Patella umbella, sp. 71. Mart. 1. t. 7 fig. 52.59. 32.St.

$94 \ldots 83$ * P. Discus. Der Teller. Gm. Patella umbella, sp. 71. var. 1 St.

95...84 P. Variabilis. Die veränderliche Patelle, wie vorhergehende, nur statt rosenroth mit braunen Streifen. Gm. P. umbella, sp. 71. var. 9 St.

95...85 P. Grisen. Die grane Patelle. Gm. P. grisea, sp.188. Adans. Seneg. 1. t. 2 fig. 5. 3 St.

97...85 P. Plumbea. Die stahlgraue Patelle. Gm. P.margaritacea, sp. 80. Niart. 1 t. 10 fig. 85 a. 10 St.

98...87 P. Silicina. Die Feuersteinfarbene Patelle. Gm. P. margaritacea, sp. 80 . Mart. 1 t. 10 fig. 85 b. 2 St.

\section{Lade 4.}

99...88 P. Gigas. Die grosse Patelle. Faranme tab. 2 fig. D.1. 5 Stiick. 
100 ..89 P. Marmorá. Die marmorweisse Patelle. Faranue tab. 2 fig. D. 2. 3 St.

101...90 P. Senilis. Die abgenutzte Patelle. Gm. Patella judica, sp. 127. Mart. 1 tab. 7 fig. 49. 1 St. $4 \frac{T}{2}$ Zoll gross. (etwas wurmigt)

$102 . . .50 *$ P. Crepidula.

103... \% - Mart. 1 tab. 8 fig. 65. 10 St.

104... $\beta$ - Faranue tab. 2 fig. B. 1. 5 St.

105... $\gamma \subset-4$ St. (Sehr schön).

106...91 P. Nodasa. Die Fnoten-Patelle. 4 St.

107...92 P. Punctulata. Die punctirte Patelle. Gm. sp. 68 \& 132. Patella punctulata, sp. 68. ITart. Conch. 1 t. 7 fig. 55. Lister 537 fig. $20 . \cdot 3$ St.

109...93 P. Aspera. Die rauhe Patelle. Favanne tab. 2 fig. G. 2. 3 St.

*109...93* P. Solaris. Die Sonneristrahlen-Patelle. Gm. P. tenuis, sp. 81. Mart. 1 t. 10 fig. 8\% 1 St. *110...93 ** P. Lunzaris. Die Mond-Patelle. 1 St. 111...94 P. Nubila. Die wolligte Patelle. $3 \mathrm{St}$. 112..95 P. Cerata. Die wachsgelbe Patelle. 1 St. 113...96 P. Rustica. 'Die geneine Patelle. Gm. P. rustica, sp. 13\%. Schröte Einleit. in Conch.' 2 p. 428 tab. 5 fig. 4. Schrüter n. Litter. 3 p. 108. 208-211. Gualti tab. 8 fig. P. Gualti tab. 9 fig. C.

$114 . . \%$ \& -8 St.

115... $\beta$ - K Korr Vergn. 3 tab. 30 fig. 5. 16 St. 116...97 P. Squamata. Die schuppigte Patelle. Gm. P: lufitanica, sp. 123. Mart. fig. 35 a. b. 2 St.

117...98 P. Granularis. Die hornichte Patelle. Gm. P. granularis, sp. 21. Mart. 1 t. 8 fig. 61. Lister Conch. 536 fig. 15. 9 St.

118...99 P.Fulva. Die rothgelbePatelle. Gm. P.fulva, sp. 102. 2S.

\section{Lade 5 .}

119..100 P. Magellanica. Die Magellanische Patelle. Gm. P. magelanica, sp. 52. Gualti test. tab. 9 fig. 8. Martini 1 tab. 5 fig. 40. a. b.

$120 \ldots \quad \alpha \div-18 \mathrm{St}$.

121... $\beta-1$ St. Wovon 2 St. abgezogen..

122...101 P. Corbis. Der Korb. Gm, P. magelanica, sp. 5? var, 1 St。 
123...102 P. Argentea. Die Silber-Patelle. Gm. P. argentea, sp. 60. Schrüter n. Litter. 3 p. 69 n. 130. 1 St.

\section{Appencliculato. Angesetzte.}

124...103 P. Coefhlear. Der Pferckekopf. Gm. sp. 155. Schröter n. Litter. 3 p. 105. n. 204 . Korr 2 tab. 25 fig. 3. 5 St. 125...104 P. Rudicula. Der Lüffel. Favamne t. 97 f. B. 1. St.

\section{Patella. Schüssel-Muschel.}

2. Imperforatce. Undurchbohrte.

*** Margine dendato. Mit gezälneltein Rand.

1. Polygonce. Vielwinklichte.

126...105 P. Medusae. Das Medusen-Haupt. Gm. P. ferua ginea, sp. 73. Knorr Vergn. 3 tab. 30 fig. 1. Mart. 1 tab. 8 fig. 65.12 St.

127...105 P. Fusca. Die dunkelbraune Patelle. Gm. P. fusca, sp. 138. 1 St.

128...107 P. MTodulus. Die Form-Patelle. Gm. P. monopis, sp. 78. Sehr selten. \&) Lister Conch. tab. 532 fig. 11. I S St.

129...108 P. Laciniosa. Die vielstrahligte Patelle. Gm. sp.18, Ynorx Vergn. 6 tab. 30 fig. 2-4. Mart. Conch. 1 tab. 10 fig. 81.2 St.

130...109 P. Lamellata. Die blattrichte Patelle. vors Born tab. 18 fig. 1. 6 St.

131...110 P. Spinetum. Die dornichte Patelle. Gm. P. barbara, sp. 20. Knorr Vergn. 5 tab. 13 fig. 5. 4 St.

\section{Lade 6.}

132...111 P. Granatina. Das Bocks-Auge. Gm. P. granatina, sp. 22. Knorr Vergn. 1 tab. 30 fig. 2. Mart. 1 t. 9 fig. 71. 72. $21 \mathrm{St}$.

133...112 P. MTunitialis. Die Festungs-Patelle. Favanne tab.2 fig. B. 2. $3 \mathrm{St}$.

134...113 P. Castrensis. Die Lager - Patelle. 9 St. $135 \ldots 113$ P. Obsidionalis, Die Hornwerks-Patelle. 11 St. 196...115 P. Canaliculata. Die ausgèhühlte Patelle. Is St. 137...116 P. Depressa. Die flache Patelle. Knnorr 4 tab. So fig. 4 . 5 St.

138...117 P. Nivalis, Die Milch-Patelle. 4 St. 
139...118 P. Sidut. Die Gestirn : Patelle,

140... $\propto-1$ Stück,

141... $\beta-2$ do.

\section{Stellata. Sternförmige:}

142...119 P. Corrusca. Die Glanz-Patelle. 1 St.

143...120 P. Radians. Die Strahl-Patelle. Knorr Vergn. 3 t. 29 fig. 3. 6 St.

144...121 P. Astrolepas, Die Stern-Patelle. Gm. P. monopis, sp. 78. Mart. 1 tab. 9 fig. 80. 4 St.

145...122 P. Polaris. Dey: Polarstern. Gualt. t. 9 fig. F. 5 St. 146...123 P. Stellaris. Der Ordenstern. Gm. sacchatina, sp.19. Lister Conch. t. 532 fig. 10. Fav. t. 2 fig. 2.3. 4 St.

\section{Lade 7. \\ Haliotis, See-Ohr.}

147.... H H. Gigas. Das grosse See-Ohr. Gm. sp. 18. gigantea. Ch. C. 10 t. 167 fig. 1610; 11. I St. gross 6 Zoll. ,

148...2 H. Elephantina. Das Elephanten-Olr.. Wurmigt.

149...3 H. Iris. Das Regenbogen-See-Ohr. Gm. sp. 19. Ch. 10 tab. 167 fig. 1619. 5 St. (3 St. abgezogen, 2 St. in Schleimhaut.)

150...4 H. IVidae. Das Midas-Ohr. Gm. sp. 1. Mait. 1 t. 14 fig. 196. Gualt. t. 69 f. B B. \& St.

151...5 H. Tuberculata. Das knollige See-Ohr. Gm. sp. \&. Mart. 1 tab. 15, 16; fig. 145-149. $13 \mathrm{St}$.

\section{Lade 8.}

152...6 H. Striata. Das gestreifte Sce-Ohr, Gm,sp.3. Nart. Conch. 1 tab. 14 fig: 138. Inorr Vergn. tab. 17 fig. 2, 3. 21 St.

153...7 H. Cochlear. Das löffelförmige See-Ohr. i St。

154...8 H. Rugosa. Das runzlichte See-Ohr. 4 St.

155...9 H. Plicata. Das gefaltene See-Ohs. Gmel. sp. 19, Schröt. Einl. in Verst. 4 p. 278 t. 3 f. 9. 2 St.

156...10 H. Rudis. Das plumpe See-Ohr. 1 St.

157...11 H. Caelata. Das gemeisselte See-Ohr. Gm. sp. 1\%, ovina. Chemn. 10 t. 166 f. 1609. 3 St.

158...12 H. Incrustata, Das gedeckte See.OHT, $2 \mathrm{St}$, 
159...13 H. Cingylata. Das gegürtelte See-Ohr. Gm. sp. \% parva, das grüne. Martsi tab. 14 fig. 140. Knorr Vergn. 1 tab. 20 fig. 5. St.

160...14 H. Fubicunda. Das rothe See-Ohr. Gm. sp. 7 parva. Knorr V. 1 tab. 20 fig. 5. Mart. 1 t. 14 f. 140 . $3 \mathrm{St}$. 161...15 H. Aleata. Das würfliche See-Ohr. Gm. sp. 9. H. australis. Ch. 10 t. 165 f. 1603,1604 . I St.

162...16 H. Virginea. Das niedliche See-Ohr. Gm. sp. 16. Ch. 10 t. 166 f. $160 \%$, 1608. 2 St.

169...17 H. Granulata. Das bucklichte See-Ohr. Gm. I. varia sp. 4. Mart. 1 tab. 15 fig. 144. 6 St.

164...18 H. Marmorata. Das gemarmelte See-Ohr. Gm. sp. 5. H. marmorata. Knorr Vergn. 2 tab. 17 fig. 4, 5. Mart. 1 tab. 14 fig. 139, 145. 10 St.

165...19 H. Picta. Das unvergleichliche See-Ohr. Gm. sp. 14. H. glabra. Ch. 10 tab. 166 fig. 1602. 1 St.

166...20 H. Asinina. Das Esels-Ohr. Gm. sp. 6. Mart. 1 t. 16 f. 150 . Knorr V. 3 t. 16 f. 1 .

$16 \%$... $\alpha_{0}-7 \mathrm{St}$.

$163 \ldots \beta_{0}-3$ St.

$169 \ldots \gamma-\beta$. $\gamma_{\text {. }}$.

\section{Lade 9.}

\section{Sinum. Milch-Näpchen.}

170...1 S. Fuscum. Das weissbraume Milch-Nüpchen. Gm. Helix. sp. 152 halioioidea. Knorr V. 4 tab. 17 f. 5. Mart. 1 tab. 16 fig. 151-154. 5 St.

171...2 S. Haliotoideum. Das See-Ohr fürmige Milch-Näp. chen. Gm. Helix haliotoidea sp. 152. Knorr Vergn. 6 tab. 39 fig. $5.5 \mathrm{St}$.

14 $172 . . . \%-1$ St.

\section{Bulla. Die Blase.}

173...1 B. Aperta. Die durchsichtige Blase. Gmel. sp. 8. Mart. C. 1 p. 266 vign. 13 fig. 3. Ch. C. 10 tab. 146 fig. 1354, 55. Gualti tab. 13 fig. EE. 4 St.

174...2 B. Naucum. Die Schwimm-Blase. Gmel. sp. 7. Hnorr Vergn. 6 tab. 39 fig. 2, 3. Mart. 1 tab. 21 fig. 200, 201. I St. 
175...3 B. Hydatis. Die Wasser-Blase. Gm.sp.9. Chemn. 9 tab. 118 f. 1019. Mart. 1 tab. 21 fig. 199. 6 St.

176...4 B. Cylindrica. Die cylinderfürmige Blase. Gm. sp. 38. Ch. t. 10146 f. 1356,57 . Lister 714 f. 70. $2 \mathrm{St}$ 177...5 B. Lignaria. Der Krollkuchen. Gm. sp. 11. Knnorr V. 6 t. 37 f. 4, 5. Mart. 1 t. 21 f. 194, 195. 7 St.

178...6 B. Umbilicata. Das lange Kibitz-Ey. Gm. sp. 10 ampulla. B. Mart. 1 t. 22 f. 202-204. 3 St.

179...7 B. Physis. Die zerbrechliche Blase. Gm. sp. 12. Argerv. C. t. 17 f. 1. Mart. 1 t. 21 f. 196, 97. 6 St. 180...8 B. Cingulata. Die gegürtelte Blase. Gm. sp. $36 \mathrm{Ve}$ lum. Ch. 10 tab. 146 fig. 1348, 49. Mart. 1 tab. 21 fig. 198. 4 St.

181...9 B. Ovum Vanelli. Das Kibitz-Ey. Gm. sp. 10 Ampulla. Mart. 1 t. 21 f. $188,189.12$ St.

182...10 B. Anıpulla Striata. Das gestreifte.Kibitz-Ey. Gm. sp. 10 Ampulla. 5 St.

183...11 B. Fasciata. Das gebändete Kibitz-Ey. Gm. sp. 10 Ampulla. Mart. 1 t. 21 f. 190,191. 5 St.

184...12 B. Rubra. Das rothe Kibitz-Ey. Gm. sp. 10 Ampulla. $14 \mathrm{St}$.

185... Is B. Flammea. Das geflammte Kibitz-Ey. Gm. sp. 10 Ampilla. 2 St.

\section{Nerita. Halbe Mond-Schnecke,}

* Claustro Glabro. Mit glatter Decke.

1. Labio crenulato, Clavicula elongata. Mit gezähnelter Lippe und verlangerter Spindel.

186...1 N. Corona. Die Dornen-Krrone. Gm. N. sp. 26. Rumpf. mus. tab. 22 f. O. Mart. 9 tab. 124 fig. 1083, 1084. 2 St.

187... 1 * N. Spinula. Die stachlichte Nerite. Gm. N. co. rona, sp. 26 var. 2.St.

188...2 N. Serpentina. Die Schlangen-Nerite. Geve tab. 24 f. 250-52. Lister 606 fig. $35.11 \mathrm{St}$.

189...3 N. Cardinalis. Die Purpur-Nerite. Gm. sp. 29 Aluviatalis. Mart. fig. 1088 h.i. 1 St.

190...4 N. Latilinea. Die breitlinigte Nerite. Gm. sp. 29 fluviatilis, Miart. 9 tab. 124 fig. 1088 a. c. 4 St. 
191...5 N. Paralella. Die gleichlienigte Nerite. Gm. sp. 29 fluviatilis. $\delta$. Mart. 9 tab. 124 fig. 1080, 81. 5 St. (2 St. mit.dem Thiere.)

192...5* N. Ziczac. Die Zickzack-Nerite, 1 St.

193...6 N: Usnea. Die mossfarbige Nerite. Lister 605 fig. 33. $1 \mathrm{St}$.

194...7 N. Arachnoidea. Die Spinneweben-Nerite. 1 St.

195...8 N. Dubia. Dic Bänder-Nerite. Gm. sp. 34. Mart. 5 tab. 193 fig. 2019, 20:0. 9 St.

196...9 N. Palella. Die Mütze. Gm. N. violacea, sp. 68، Mart. 1 tab. 13 fig. 133, 134. $4 \mathrm{St}^{2}$

$197 . . .10$ N. Hians. Die weitmündige Nerite. Lister 604 fig 24. 1 St.

198...11 N. Ocuins. Die Augen-Nerite. Gm. sp. 35 pulligera. Mart. 9 tab. 124 fig. 1078, 79. 2 St.

799...12 N. Crocea. Die Saffran-Nerite. Geve tab. 23 fiğ 248 a $\& \mathrm{~b}$

\section{Lade 10 .}

2. Labio deniato, clacicula depressa. Mit gezähnelter Lippe und gedrückter Spindel.

200..13 N. Fusco fasciata. Die rothe Band-Nerite. $2 \mathrm{St}$ 201...14 N. Albo Lineata. Die weisse Band-Nerite. 1 St. 202..15 N. Nigro cincta. - Die schwarz gebandete Nerite. Mart. 9 tab. 124 fig. 1089 Litt. X. 2 St.

203...16 N. Olivacea. Die gelb gebandete Nerite. $2 \mathrm{St}$. 201...17 N. Polita. Die bunte Nerite. Gm. sp. 43. Geve tab. 22 fig. 224 a \& b. Innorr Vergn. Ster Theil tab. 1 fig. 4. 5 St.

$205 \ldots 18 \mathrm{~N}$. Fulminea. Die geflammte Nerite. $1 \mathrm{St}$. $206 \ldots 18$ * N. Nebulosa. Die wolkigte Nerite. $3 \mathrm{St}$. 207...18** N. Fascia lata. Die breite Binde. 1 St. 208..19 N. Dendrites. Die gemahlte Nerite. 1 St. $209 . .20$ N. Monile. Die Ketten-Binde. 1 St. 210..21 N. Aurantiaca. Die Orange-Nerite. 1 St. 211...22 N. Opaca. Die helldunkle Nerite. Gm. sp. 43 polita. $1 \mathrm{st}$.

$212 . \ldots a_{0}-20 \mathrm{St}$.

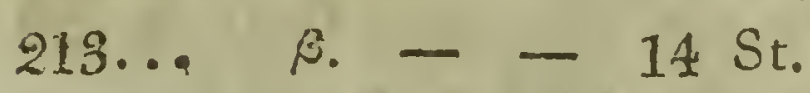

214.. $\gamma_{:}-$- Ch. 5 tab. 193 fig. 2006, \%. 4 St. 
215...23 N. Aspersa. Die bestaubte Nerite. 3 Se. 216...24 N. Chrysostoma. Die rothmündige Nerite. 6 St. (3 St. mit Decken.)

217...25 N. Arriaca. Der schwarze Flor. Gm. sp. 66 bifas. ciata. Ch. 5 tab. 193. 2015. 1 St. mit Deckel.

\section{Nerita. Halbe Mond Schnecke.}

** Claustro muricato. Mit geperlter Decke.

Labio trenulato rugoso clavicula clepressa. Mit gezähnelter und gerunzelter Lippe und gedrückter

\section{Spindel.}

218...26 N. Alabastrina. Die weisse Nerite. 1 St.

219...27 N. Variegata. Die schüchigte Nerite. Gm. sp. 48 Grossa. Geve tab. 23 fig. 239 a \& b. Mart 5 t. 191. 1958, 69. 5 St.

220...2S N. Nubecula. Die russfarbene Nerite. 2 St. 221...29 N. Angulata. Die winkligte Nerite. Gm. sp. 65 tessellata. Cl. 5 t. 192. 1998, 1999. 2 St. 222...30 N. Imperfecta. Die unvollendete Nerite. Gm. sp. 45. Albicilla. Ch. 5 t. 193.2000 a. b. c. d. $15 \mathrm{St}$.

\section{Nerita. Halbe IMond-Schnecke.}

*** Claustro rugoso muricato. Mit gerunzelter und geperlter Decke.

Labia rugoso-dentaro clavicula elongata. Mit gerunzelter und gezähnelter Lippe, verlängerter Spindel. 223...31 N. Péloronta. Die aufgeblasene Nerite. Gm. sp.44. $\beta_{\text {. }}$ 2 Stück.

224...32 N. Papilio. Der Schmetterling. Gmel، sp. $444_{-}^{\circledR P e l o}$ tano Chemn. 5 tab. 92. 197\%. 2 St.

225...33 N. Cruenta. Die blutige Nerite. 2 St.

226...34 N. Virginea. Die Blumen-Nerite. Gm. sp. 44. Pe= lotonta Ch: 5. tab. 92. 1978-1980. 20 St.

227...35 N: Musica. Die Noten-Nerite. Gm. sp. 63. Alammea Chemn. 5 tab. 192. 1994. 7 St.

228...36 N. Tessellata. Die würfligte Nerite, Gmel. sp. 58. Pica Chemn. 5 tab. 191. 1964, 1965. I St.

229...37 N. Mille punctata. Die tausendfacls punctirte Nerites 1 Stück.

230...38 N. Fascia. Dio Binde. I St. 
231...39 N. Maculara, Die gefleckte Nerite. 2 St.

2:2...40 N. Pulchra. Die schöne Nerite. 1 St.

233...41 i. Nigrita. Die Neger-Nerite. Gm. sp. 46. Histrio Mart. 5 tab. 191 fig. 1960. 1961. 11 St.

235...42 N. Conspurcata. Die beschmutzte Nerite. 1 St.

235...43 N. Chamaeleon. Die vielfarbige Nerite. Gm. sp. 49.

Chemn. 5 tab. 192 fig. 1988, 1991. Regenf. tab. 3 fig. 26. 1 St.

237...44 N. Muricata. Die stachligte Nerite. 2 St.

238...45 N. Pecten. Die Kamm-Nerite. 1 St.

${ }^{*} 239 . .46 ! !$ N. Schmideliana finistrorsa, fossilis. Die linksgewundene Schmidelsche Nerite. Gm. sp. 72. Chemn. C. 9 tab. 114 fig. 975, 76. Naturf. 6 p.165. 2. St.

\section{Lade 11.}

240...47 N. Nigreola. Die Ebenholz-Nerite. Gmel. sp. 62. Antillatum. Chemn. 5 tab. 192. 1987. I St.

241...48 N. Eburnea. Die Elfenbein-Nerite. Gm. sp. 47 plicata. Favanne tab. 10 fig.Q.3. Lister 595 fig. 3. 5 St.

\section{Albula. Halb-Nerite.}

\section{* Testiculatae. Hodenartige.}

243..1 A. Albumen. Die eyweisse Halb-Nerite. Gm. Nerita, sp. 5. Mart. 5 tab. 189. 1924, 25. Knorr V. 4 tab. 7 fig. 4, 5. 3 St.

214...2. A. Vitellus. Der Eydotter. Gm. N. orientalis, sp.12. Chemn. 5 tab. 188. 1899, 99. 8 St.

245 ...3 A. Didyna. Der braune Hoden. Gm. N. glaucina, sp. 3. Favanne tab. 10. Lit. K. L. Chemn. 5 tab. 186 fig. 1856, 57. 6 . St.

246...4 A. Mrammilla. Die Zitzen-Halb-Nerite. Gm. N. sp.6. Chemn. 5 tab. 189. 1928-31. 9. St.

247..5 A. Adusta. Die angebrannte Halb-Nerite. 2 St.

248...6 A. Aurantium. Die Pommeranze. Gmel. N. melanostoma, sp. 19. A. Chcmn. 5 tab. 189 fig. 1994, 1995. Innorr Vergn: 4 tab. 6 fig. 3: 4. 2 St.

\section{Albula. Halb-Nerite.}

** Umbilicatae. Genabelte.

240...7 A. Hepatica. Die blasgelbe Halb-Nerite. Gm. N. melanostcma, sp. 19. Ch. 5 tab. 189 fig. 1932, 39. 1- St. 
250...8 A. Mammata. Die Brust-Drüse. Gm. N. melanostoma, sp. 19. $\gamma$. Chemn. 5 tab. 189 fig. 1936, 37. 7 St.

251...9 Fasciata. Die gebandete Halb-Nerite. Gm. N. melanostoma, sp. 19. $\varepsilon_{0}$ Ch. 5 tab. 189 fig. 1940, 41. 1 St. 252...10 A. Tranquebarica. Die gläserne Halb-Nerite. Gmel. N. Papilla, sp. 20. Ch. 5 tab. 189. 1939. $2 \mathrm{St}_{\text {, }}$

\section{Cyphoma. Halb-Porcellane.}

253...1 C. Gibbosa. Der Höcker. Gm. Bulla, sp. 6. Mart.1 tab. 22 fig. 213, 14. Knorr 1 tab. I4 fig. 4. 13 St.

254...2 C. Dorsata. Der Hochrücken. Gm. Bulla gibbosa, sp. 6. Mart. 1 tab. 22 fig. 211, 12. Knorr 1 tab. 14 fig. 3. 5 St.

\section{Volva. Das Ey.}

255...1 V. Cygnea. Das Schwanen-Ey, mit brauner Mündung. Gm. Bulla Ovum, sp. 1. Mart. 1. tab. 22. fig.205, 206. 6 Stïck.

256...2 V. Ovum. Das Gänse-Ey, dünnschaligt, mit weisser Mündung. Gm. Bulla ovum, sp. 1. 5 St. 257...3 V. Hypenemium. Das Wind-Ey. 1 St.

*258..4 !! V.Birostris. Die falsche Weberspuhle. Gmel. Bulla, sp. 3. Mart. 1 tab. 23. 217. a. b. Lister 711 fig. 66. 1 Stück.

*259..5!! V. Textoria. Die äclite Weberspuhle. Gm. Bulla Volva, sp. 2. Mart. 1 tab. 23 fig. 218. Knorr Vergn. 5. tab. 1 fig. 2, 3, 6 tab. 32 fig. 1. Selten ùnd schön. 4 Zoll lang.

260...6 V. Perla. Die Perle. Gm. B. verrucosa, sp. 5. Mar. tini 1. tab. 23 fig. 220, 21. Lister 712 fig. 67. Hnort Vergn. 4 tab. 26. fig. 7. 6 St.

\section{Cyprcea. Die Porcellane. \\ * Glabosae. Kugelfärmige.}

1. Trregulares. Unausgewachsene.

261.... C. Ovum. Das Hüner-Ey. Gm. Bulla ferruginosa, sp. 35. Mart. 1 tab. 22, 2c9. $\beta$. 1 St.

262...2 C. Nivea. Das Tauben-Ey. 2 St. 262...3 C. Limbata. Die gesïumte Porcellane. I St: 
264...4 C. Fulliginosa. Das geflammte Ey. Gm, B. ferrugi. nosa, sp. 35. Mart. 1 tab. 22 fig. 209, 210. 2 St.

265...5 C. Paschalis. Das Oster-Ey. Gm. Bulla ovata, sp.34. Mart. 1 tab. 22 fig. 207, 208. Knorr Vergn. 6 tab. 18 fig. 2 tab. 27 fig. 6.2 St.

256...5* C. Prunus. Die flaumenförmige Porcellare. Gmel. C. Pulla, sp. 61. Mart. 1 t. 26 f. 269, 70. 2 St.

$26 \% \ldots 5^{* *}$ C. Bullata. Die gebandete Porcellane. Gm. C. cinetea, sp. 16. Mart. 1 t. 25 f. 254, 55. 1 St.

268...6 C. Carthaginensis, Das Klip-Horn. Gm. C. Mus. sp. 43. Mart. 1 t. 23 f. 222, 23. Knorr V.3 t. 12 f.3. 3 St. 269...7 C. Tuberosa. Die buckligte Porcellane. 3 St:

270..8 C. Mauritiana. Der grosse Schlangen'-Kopf. Gmel, Cypr. sp. 41. Knorr 2 tab. 27 fig 5. Mart. 1 tab. 30 fig. 317.319 .

\section{Lade 12.}

$271 \alpha-\infty$ Sto $_{0}$

$272 \beta-2 \mathrm{St}$.

$273 \gamma-\beta-3 \mathrm{St}_{0}$

274...9 C. Castanea. Die Kastanienbraune Porcellane. Gmel, C. sp. 41 var.

275...10 C. Caput Serpentis. Der kleine Schlangenkopf. Gm. C. sp. 39. Knorr 4 t. 9 f. 3. Mart. 1 t. 30 f. 316. $23 \mathrm{St}$.

276...11 C. Oculata. Die vieläugigte Porcellane. Gmel. C. Histrio, sp. 120. Chemn. 10 t. 145 f. 1346, 47. Favanne 3 t. 12 fig. F 2. 2 St.

277...12 C. Histrio. Der Harlekin. Gm. sp. 120. Finorr V. tab. 16 fig. 1. 2 St.

278...13 C. Stellata. Die gestippelt Porcellane. Gm. C. Helvola, sp. 90. Mart. 1 t. 30 f. 320̂, 27. List. Conch. f. 38. 14 St.

279...14 Stercoraria. Der Hochrücken. Gm, sp. 6. Mart. 1 tab. 31 fig. 33\%. 6 St.

280...15 C. Ligata. Die bebandete Porcellane. Gm. C. Ster coraria, sp. 6.1 St.

281...16 C. Crenata. Die Gekerbte Porcellane. Gm. C. cau rica, sp. 83. Mart. 1 tab. 29 fig. 303. \& St.

282...17 C. Lentigo, Die Sommersprosse. 'Gm. C.: caurica, sp. 83. 1 St. 
283...18 C. Annulus. Die Ring-Porcellane. Gm. C. sp. 82. Mart." 1 tab. 24 fig. 239, 240. 18 St.

284...19 C. Mcneta. Die Mïnze. Gm. C, sp. 81. Mart. I tab. 31 fig. 339, 40. 4 St.

285...20 C. Numisma. Die Pfennings-Porcellane. Gm.C. nioneta, sp. 81. Mart. 1 f. 337; 38.5 St.

285...21 C. Variolosa. Die Blätter-Porcellane. Gm. C. nu• cleas, sp. 95. Mart. 1 tab. 29. 318. Knorr \& tab. 17 fig. 7. $6 \mathrm{St}$.

28\%...21 * C. Lima. Die Feile. Gm. C. sp. 95. var. 3 St.

288...22* C. Oniscus. Der Kellerwurm. Gm. C. sp.93. Tra 3 Stück.

289...23 C. Pedicutus, Die Laus. Gm. C. sp. 93. Martini 1 tab. 29. 310, 11. 2 St.

290...24 C. Pulex. Der Floh. 2 St.

\section{Regulares. Ausgewachsene.}

291...25 C. Canaliculata. Die dünnschaligte Porcellane. Gm, sp. 14. fragilis. Gnalti, tab. 16 fig. G. G. $6 \mathrm{St}$.

292...26 C. Cruentata. Die blutrothe Porcellane. Gmel. C. squalina, sp. 101. Das Emmerlings-Ey. Gualti, t.16. fig. R. Mart. 1 t. 25 fig. 250, 51. $6 \mathrm{St}$.

\section{Lade 13.}

293...27 C. Onea. Gm. C. tigris, sp. A4. Mart. 1 tab. 24 fig. 234. $1 \mathrm{St}$.

294...23 C. Tigris. Die Tieger-Porcellane, Gm. C. sp. 44. Mart. 1 tab. 24 f. $232,33.14 \mathrm{St}_{\text {. }}$

295...29 C. Pardus. Die Parder-Porcellane. Gm. C. sp. 44. Mart. 1 tab. 24 f. 235. 6 St.

296...30 C. Pardalis. Der Parder. Gm: C. sp. 44. 5 St.

297...31 C. Lynx. Die Lux-Porcellane. Gm. C. sp.48. Mart. 1 tab. 23. 230.31. $11 \mathrm{St}$.

298...32 C. Vitellus. Die weissgeflechte oder Salzkörner Por. cellane. Gm. C. guttata, sp. 15. Mart. 1 tab. 23 fig. 228. 29. 13 St.

299... $\alpha_{0}$ - - Mart. 1 tab. 25 fig. 252. 1 St.

300...33 C. Ocellata. Die geäugelce Porcellane. Gm. sp. 91. Mart. 1 tab. 31 fig. 393, 34. 4 St.

301...34 C. Coeca. Die bliride Porcelane. Gm. Cypr. poraria, sp 92. - 3 . Mart. 1 t. 24 f. 23\%. I St. 


\title{
Lade 1/4.
}

302...55 C. Ziczack. Die Zickzack-Porcellane. Gm. C. sp. 54. Mart. 1 tab. 23 f. $22 \hat{5}, 2 \%$. 3 St.

303...36 C. Globulus. Die Kugel-Porcellane. Gm. C. sp 99. Mart. 1. tab. 24. fig. 242. Knorr 6 t. 21 fig. 7. 1 St.

\section{Ciprcea. Die Porcellane.}

\author{
** Cylindracaea. Walzenfürmige. \\ 1. Mucronata. Zugespitzte.
}

304...37 C. Testudinaria. Die Schildpatt-Porcellane. Gm. C. sp. 5. Mart. 1 tab. 27 fig. 27L, 72. 3 St.

305...38 C. Meleagris. Die Argus-Porcellane. Gm. C. sp. 1 exanthema. Mart. 1 tab. 26 fig. 25\%, 258. Chemn. 10 t. 145 f. 1343 B. 2 St.

$306 . . .-\alpha_{0}$ - Knorr 1 tab. 5 fig. 3. 2 St.

$307 \ldots-\beta_{0}-4 \mathrm{St}$.

$308 \ldots-\gamma_{0}-$ - 8 St. davon 2 Stïck abgezogene.

$309 \ldots-\delta . \quad-$ Knorx 2 tab. 24 fig. 2. 1 St.

310...39 C. Mappa. Die Lanàkarten-Porcellane. Gm. C.sp. 2. Mart. 1 tab. 25 fig. 245, 46. Favanne tab 29 fig. A. 3. 5 St.

311...40 C. Arabica. Die arabische Buchstaben-Yorcellane, Gm. C. sp. 3.

312...- - . - - Mart. 1 tab. 23 fig. 323, 29. 20 St. $313 . . .-\beta_{0}-2 \mathrm{St}$.

\section{Lade 15.}

314...41 C. Argiolus. Der kleine Ajgus. Ch. 10 tab. 144 fig. 1338 a \& b. 3 St.

315...42 C. Argus. Der ächte Argus. Gm. C. sp. 4. Mart. 1 tab. 28 295, 86. Cl. 10 t. 145 f. $1344,45.8$ St.

316...43 C. Glanca. Der Maulwurf. Gm. C. plumbea, sp. 17 dickschaligt. Mart. 1 t. 26 f. 255. 1 St.

317...44 C. Zebra. Der Kapsche Esel. Gm. sp. 17 plumbea, dünnschaligt: Mart. 1 t. 26 f. 256 . 8 St.

318...45 C. Carneola. Die rothe Fuchs-Porcellane. Gm. sp.

7. Mart. 1 tab. 28 fig. 237, SS. 12 St.

319...46 C. Talpa. Die rothe Maus. Gm. sp. 9. Mart. I זab. 27 fig. $273,74.5$ St. 
320...47 C. Lurida. Die miesfarbigte Porcelline. Gm. sp. 11. Mart. 1 tab. 30 fig. 315. 2 St.

321...48 C. Soricina. Die Spitzmaus. 2 St.

\$22...49 C. Isabella. Die isabelfarbene Porcellane. Gm. sp. 49. Mart. 1 t. 27 f. 275 . Knorr 4 t. 9 f. 5.4 St. 323...50 C. Pyrum. Die birnförmige Porcellane. Gm. sp. 59. Mart. 1 t. 26 f. $26 \%$, 68. Knorr 2 t. 5 f. 5 . 11 St. 324...51 C, Undosa. Die gewellte Porcellane. Gm. Amethy. stea, sp. 10. Mart. I. t. 25 f. 247-249. Knorr V. 5 tab. 28 fig. $5.5 \mathrm{St}$.

S25...52 C. Amethystea. Die amethistfarbene Porcellane, wie No. 5 S nur abgeschliffen. 1 St.

\$26...53 C. Crenulata. Die angeschnittene Porcellane. 1 St.

\section{Umbilicata. Genabelte.}

32\%...54 C. Rubiginosa. Die rostfarbene Porcellane. 1 St. 328...55 C. Interrupta. Die Türkischpapier-Porcellane. $1 \mathrm{St}$. 399...56 C. Petechialis. Die Flecken-Porcellane. Gm. C. sane guinolenta, s. 38. Mart. 1 t. 26 f. 265, 66. 1 St.

390...57 C. Stolida. Die Oliven-Porcellane. Gmel. sp. 89. Mart. 1 tab. 28 fig. $292,93.10 \mathrm{St}$

\section{Lade. 16.}

S31...55 C. Oliva. Der Olivenkern. 4 St.

322...59 C. Cornus. Die Kornele. Gm. Cypr. Stolida, sp. 89. B. Mart. tab. 28 fig. 294, 95. 1 St.

339...60 C. Draco. Die Drachen-Porcellane. Gm. C. Rubiginosa, sp. 105. Mart. 1 t. 29 f. 305. 1 St.

334...61 C. Quinque fasciata. Die fünfbandige Porcellane. Gm. C. Stolida, sp. 89. Mart. 1 t. 28 f. 292, 93. 4 St. 335...62 C. Morbillosa. Die Masern-Porcellane. Gm. C. cautica, sp. 83. \%. Mart. 1 t. 29 f. 303. 7 St.

336...63 C. Erosa. Die gebeitzte Porcellane. Gmel. sp. 34. Mart. 1 tab. 30 fig. 320, 21. Knorr 6 tab. 20. fig. 4. 13 St. (2 St. abgezogen.)

337...64 C. Cribraria. Der Goldtropfer. Gm. sp. 80. Mart. 1 tab. 31 fig. 335. 6 St.

388...65 C. Asellus. Das Eselchen. Gm. sp. 56. Mart. 1 tab. $27 \mathrm{fig} .280 .11 \mathrm{St}$.

330...65 C. Ovum Passeris. Das Sperlings-Ey. 6 St. 


\section{Cassis. Die Sturmhaube.}

* Verae. Acchte.

1. Triangulares. Dreyeckigte. Labio lamellato. Mit blattrigter Lippe.

S10...1 C. Caput-equinum. Der Pferdekopf. Gm. Buccin cornutum, sp. 11. B. Mart. 2 tab. 35 fig. 362. Lister 1003. 2 St. extra gross.

$3+1 . .2$ C. Hamata. Die Tussangel. Gm. Buccin cornutum, sp. 11. \% Lister 1009 fig. 71 c. 2 St.

$342 . . .3$ C. Caput Bovis. Der Ochsenkopf. \& St.

$3 \nmid 3 . . .4$ C. Tuberosa. Die knolligte Sturmliaube. Gm. Buccin, sp. 13. Mart. 2 tab. 34 fig. S59. Knorr Vergn. 6 tab. 18 fig. 1.4 St.

$344 \ldots 5$ C. Cornuta. Die gehörnte Sturmhaube, Gm. Buccin cornutum.

345...- $\alpha_{0}$ - - Mart. 2 t. 33 f. $348,49.1$ St.

S46...- - . - Mart, 2 t. 35 f. 362. 1 St.

$\$ 47 \ldots 5$ * C. Pullus. Die Koffer-Sturmhaube. Gm. sp. 43. Buccin. Mart. 2 tab. 41 fig. 415. hnorr Vergn. 6 tab. 22 fig. 5. 5 St.

\$48...5 ** C. Callosa. Die schwicligte Sturmlaube. Gmel. sp. 43. Buccin. Knorr 6 t. 22 fo 6. $2 \mathrm{St}$.

\section{Fornicatae. Gewólbte.}

Labio incrassato. Mit verdickter Lippe.

s49...6 C. Rufum. Die rothe Sturmhaube. Gm. Buccin sp. 12. MIart. 2 t. 32 f. 341. Knorr V. 2 t. 9 f. 2.5 St.

350...7 C. Rufescens. Dit blutrothe Sturmhaube. Gm. Buccin, sp. 12. Mart. 2 tab. 33 fig. 36 \%, 47. Knorr $V$. 2 t. 9 f. 2 \& 4. t. 1.1 St.

$\$ 51 \ldots 8$ C. INarmorata. Die gemarmelte Sturmliaube. Gn. Buccin tuberosum, sp. 13. Mart. 1 tab. 38 fig. 381, 82. Knorr Vergn. 3 t. 10 f. 1, 2. 2 St.

$552 . . .9$ C. Flammea. Die geflammte Sturmlıabe. Gm. Buccin. sp. 14. Mart. 2 tab. S4 fig. 35s, 54. Knorr V. 4 tab. 4 fig. 1. 8 St.

353 ... - - . 0 - 1 St.

Sร̌st...10 C. Tuberculosa. Die warzige Stuxmhaube. Favanne tab. 26 fig. 11. 2 St. 
\$55...11 C. Plicatun. Die gefaltete Sturmhaube. Gm. Buccin. sp. 10. Mart. 2 t. 37. 379, 80. Lister Conch. tab. 1002 fig. 67. 1 St.

356...12 C. Pileolus. Der polnische Huth, (davon 3 St. dürnmschaligte.) Gm. Buccin. testiculum, sp. 15. Mart. 2 t. 37 f. 375, 76. Knorr Vergn. 4 t. 6 f. 1. 14 St. - - u. Mart. 1 t. 37 f. $377,78.1$ St.

\$57...13 C. Mitella Polonica. Die polnische Mütze. Gmel. Buccin testiculum, sp. 15. Mart. 2 t. 37 f. 375, 76. 2St. 358...14 C. Coronata. Die gekrönte Sturmhaube. Gm. Bucc. tessellarum, sp. 20. Mart. 2 tab. 36 fig. 369 \& 37 fig. $34 \% .2 \mathrm{St}$.

359...15 C. Pennata. Das türkische Papier. Gmel. Buccin. Pennatum, sp. 21. Mart. 2 t. 36 fig. 372, 73. Rumpf tab. 23 fig. C. 3 St.

\section{Cassis, Die Sturmhaube. \\ ** Spuriae. Unächte.}

1. Globosce. Kugelförmige. \%. Caudatae. Geschwänzte.

350...16 C. Tyrrhenum. Die tyrrhenische Sturmlaube. Gm。 Buccin. sp. 180. Mart. 10 tab. 153 f. 1461, 62. 2 St. 361...17 C. Echinophora. Der Ygel. Gmel. Buccin. sp. 9. Mart. 2 tab. 41 fig. 40\%. Knorr Vergn. 1 tab. 17 fig. 1. 4 Stück.

\section{Lade 17.}

$362 \ldots-\omega_{0}-1$ St.

363...18 C. Brunnea. Die branne Sturmlaube. Gm. Buccin. echinophora, sp. 9. $\delta$. Lister t. 1011 f. 71.5 St.

B. Simplices. Einfache.

364...19 C. Glauca. Die Bezoar-Sturmlanube. Gm. Buccin. sp. 35. 14 St.

- $\alpha$. Eine sehr dünnschaligte dito. Mart. 2 tab. $32 \mathrm{ffg}$. 342, 43. 1 St.

365;..20 C. Cancellata. Die gegitterte Sturmhaube. Gmel. Buccin. sp. 79. 2 St.

365...21 C. Guttata. Die weissgefleckte Sturmlanube. I St. $367 \ldots-\omega_{0}-1$ St. 
358...21* C. Pomum. Die apfelförmige Sturmhaube. Gmel. Buccin. sp. 4 Mart. 2 tab. 36 fig. 370, 71. 6 St.

-...21 ** Dünnschaligte dito. Lister 1016 fig. 75. 1 St.

359...22 C. Areola. Die Gartenbeets-Sturmhaube. Gm. Buccin. sp. 17. Mart. 2 tab. 32 344, 45. Knorr Vergn. 3 tab. 8 fig. 5. 1 St.

- ..22 «. - Weisse dito. 1 St.

370...23 C. Malum. Die Apricose. Gm. Buccin. sp. 17 . Lister 1056 fig. 9. 3 St.

371...24 C. Globulus. Die kugelförmige Sturmhaube. Gm. Buccin. Gibbon; sp. 24. Mart. 2 tab. 34 fig. 351, 52. Lister 999 fig. 64. $2 \mathrm{St}$.

372...25 C. Cepa. Die Zwiebel. Gmel. Buccin gibbum, sp. 24. 1 St.

373...26 C. Glans. Die Eichel. Gmel. Buccin abbreviatum, sp. 181. Mart. 10 tab. 153 fig. 1463, 64. 3 St.

\section{Elongatce. Vèrlängerte.}

a. Planiusculae. Gedrückte.

374...27 C. Alea. Das. Bretspiel. Gm. Buccin areola, sp. 17. Mart. 2 t. 34 f. 355, 56. Knorr V. 3 t. 8 f. 5.5 St.

\section{Lade 18.}

375...29 C. Cancellata. Die gegitterte Sturmhaube. Gm. Buccin decussatum, sp. 16. Mart. 2 tab. 35 fig. 360, 61. Knorr Vergn. 2 tab. 10 fig. $S \& 4$. 5 St.

375...29 C. Flammeolum. Die Brand-Sturmliaube. Gmel. Buccin decussatum, sp. 16. Mart. 2 t. 25. 367, 68. 2 St. $\$ 77 . .30$ C. Flammifera: Die gestreifte Sturmhaube. Gmel. Buccin strigatum, sp. 179. Mart. 10 tab. 153 fig. 145\%, 58. 3 St.

Sis...31 C. Rugosa. Die runzlichte Sturmhaube. Gm. Bucc. plicatum, sp. 10. Mart. 10 t. 153 f. 1459, 60. Lister 1014 fig. 78. I St.

ß. Labio columella involuto breviori.

379...32 C. Denticulata. Die gezähnelte Sturmhaube. Gmel. Buccin erinaceus, sp. 34 . Mart. 2 t. 35 f. 363. Sto $380 \ldots-\alpha_{0}-\mathrm{ESt}$.

\$31...33 C. Glatra. Die geglättete Sturmhaube. Gm. Bucc, erinaceus, sp. $\supset 4$ f. Iister 1013 f. 77. 1 St. 
382...34 C. Vibex. Die geaderte Sturmhaube. Gm. Buccin, sp. 35. Mart. 2 tab. 35 fig. 36, 65 . Knorr Vergn. 6 tab. 11 fig. 3. 4 St.

$383 \ldots-\alpha_{0}-3$ st.

284...35 C. Erinaceus. Die Ygel-Sturmhaube. Gm. Buccin, sp. 34. Mart. 2 t. 35 363. 9 St.

385... $\alpha_{0}-2 S t_{\text {. }}$

\section{Porphyria. Die Dattel.}

* Cilyndroideae. Zilinderförmige.

1. Clavicula depressá. Mit gedrückter Spindel. 386...1 P. Porphyria. Die Portobello-Dattel. Gm. Voluta, sp. 16. Mart. 2 tab. 47 485, 86. Knorr Vergn. 1 t. 15 fig. 1. 7 St.

387...2 P. Fasciata. Die gebandete Portobello-Dattel. Gm. Voluta, sp. 16. $\%$. 1 St.

388...3 P. Ruffina. Die rothe Dattel. Gm. Vol. sp. $49 \%$ 2 Stück.

389...4 P. Labradoriensis. Die Schiller-Dattel. Gm. Voluta Oliva, sp. 17. Lister 731 fig. 20. 2 St.

390..5 P. Sericea. Das Seidenzeug. Gm. Voluta Oliva, sp. 17. of. Mart. 2 tab. 51 fig. 559, 561. 8 St.

$391 . .6$ P. Miniacea. Das Morgenroth. Gm. V. porphyria, sp 16. $\beta$. Mart. 2 t. 45 f. $476,77.9$ St.

392...7 P. Coerulea. Die himmelblaue Datiel. Gm. V. Oliva, sp. 17 a. Mart. 2 t. 48 f. 518 . Rumpf t. 39 f.5. 13 St.

\section{Lade 19.}

393...8 P. Variegata. Die schächigte Dattel. Gm. Voluta, sp. 1\%. $\delta$. Mart. 2 t. 45 f. 478 , 79. 24 St.

394... $\alpha_{0}$ - - Mart. 2 tab. 45 fig. 480, 81. Knioir $V$. 5 tab. 19 fig. 1. 6 St.

395...9 P. Variabilis. Die spielende Dattel. Gm. V. Oliva, sp. 17. 7 St.

396...10 P. Reticulata. Die Netz-Dattel. Gm. Vol. Oliva, sp. 17. a. Mart. tab. 48 fig. 512, 5339 St.

397...11 P. Punctata. Die gestippelte Dattel. Gm. V. Oliva, sp. 17. 4 St.

393...12 P. Ornata. Die geschmückte Dattel. Gm. V. Oliva. sp. 17. $2 \mathrm{St}$. 
399...13 P. Amoena. Die Iubsche Dattel. Gm. Vol. Oliva, sp. 1\%. 3 St.

400...14 P. Hepatica. Die lichtgraue Dattel. Gm. V. Oliva, sp. 1\%. 2 St.

401...15 P. Isabella. Die isabellfarbene Dattel. Gm. Voluta Oliva, sp. 17. 3 St.

402...16 P. Aurata. Die goldgelbe Dattel. Gm. Vol. Oliva, sp. 17. 1 St.

403...17 P. Sepultura Principis. Das Prinzen-Begräbniss. Gm. V. Oliva, sp. 17. Rumpf t. 39 f. 4.

- $-\alpha_{0}-2$ St.

405... - - . - Mart. 2 t. 61 fig. 563. Knorr 5 tab. 26 fig. 4. 4 St.

406...- - - Mart. 2 t. 45 f. 480, 81. Knorr 5 t. 19 f. 1. 2 St.

$407 \ldots-\delta_{.}-4$ St.

408... - $\varepsilon_{0}-2$ St.

$409 \ldots-\zeta$. - -1 St.

410...17* P. Oliva. Die olivengrïne Dattel. Gm. Voluta, sp. 17. Mart. 2 t. 45 f. 503, 50t. 13 St.

-..18 P. Fimbriata. Eine schöne rostfarbigte Dattel mit dem Saum. 1 St.

411...19 P. Fasciata. Die Band-Dattel. Gm. Voluta, sp. 17: Knorr 3 tab. 17 fig. 3. 6 St.

412...20 P. Vidua. Die ungarische Wittwe. Gm. V. sp. 17. Mart. 2 t. $45 \mathrm{f} .472,73.17 \mathrm{St}$.

$413 . .1-\alpha-1 S t$.

$414 \ldots-\beta-\beta-1$ St.

415...21 P. Cingulata. Die gegürtelte Dattel. Gm. V. Oliva, sp. 17. 1 St.

416...21* P. Turgida. Die wulstige Dattel, Gm. V. Oliva, sp. 1\%. 1 St.

417...22 P. Fenestrata. Die gegitterte Dattel. Gm. V. Oliva, sp. 17 ß. Mart. 2 t. 47 f. 502.2 St.

418...23 P. Quercina. Die Eichenholz-Dattel. Gmel Voluta Oliva, sp. 17. Knorr Vergu. der Augen 5 tab, 26 fig. 4. 6 St.

$419 \ldots-\alpha_{0}-2 S t$.

$420 \ldots-\beta_{.}-$- Knori 5 tab. 27 fig. 5. $1 \mathrm{st}$. 
2. Clavicula elevata. Mit hervortretender Spindel. 421...24 P. Lutaria. Die gestreckte Dattel. Gm. V. utriculus, sp. 19 n. Mart. 2 t. 50 f. $549,50,53$. Knorr 8 t. 17 f. 2.6 St.

422...24* P. Rubicunda. Die röthliche Dattel. Gm. V. utri* culus sp. 19. $1 \mathrm{St}_{\mathrm{o}}$

\section{Lade 20.}

423...25 P. Spicata. Die Kornähre: Gmel. V. oliva sp. 17 . Mart. 2 tab. 48 fig. 509, 10. 7 St. 424...25 P. Griseola. Die gräuliche Dattel. 3 St. 425 ...27 P. Undulata. Die wellenförmige Dattel. $18 \mathrm{St}$. 426...28 P. Oculatá. Die geäugelte Dattel. $10 \mathrm{St}$. 427 „..29 P. Dealbata. Die schneeweisse Dattel. Knorr 6 tab. 34 fig. $4,5,6$ St.

$428 . .:-a-1 \mathrm{St}_{\mathrm{t}}$

429...30 P. Turbinata. Die gewirbelte Dattel. Gm. sp. 22 V. nivea. Mart. 2 tab. 50 fig. 55s. 4 St. 430...31 P. Conoidea. Die kegelförmige Dattel. 1 St. 411...32 P. Ispida. Die blaumündige Dattel. Gm. Voluta sp. 23. Mart. 2 tab. 49 fig. 521, 25, 30. Knorr \& tab. 19 fig. 3. $21 \mathrm{St}$.

432... \% - - Mast. 2 tab. 49 fig. 535. 3 St.

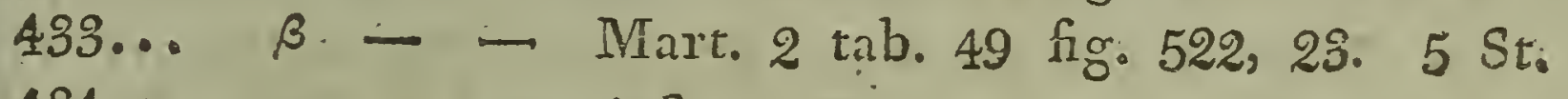

$434 . . . \quad y=-4$ St.

$435 \ldots \delta-=3 S_{t}$.

436...33 P. Mita. Die blaugefleckte Dattel. Gm. sp. 23 V. ispidula. '23 St.

$437 \ldots$... - - Mart. 2 tab. 49 fig. 527-29. \& St。

$438 \ldots \beta-10 \mathrm{St}$.

A39... $\gamma-3 \mathrm{St}_{\mathrm{i}}$

440...34 P. Amethystina. Die amethystfarbene Dattel. Gmel. sp. 23. V. ispiaula. Mart. 2 to 46 f. 491 , 92. Knorr Vergn. 2 tab. 10 fig. 6, \%. 14 St. $441 . . .35$ P. Tigris. Die Tiger-Dattel. 1 St. 442...36 P. Paleacea. Die Strohfarbene Dattel. i St. 443...37 P. Papyracea. Die buntpapierne Dattel. I St. 444...38 P. Vellus aureum: Das goldene Vliess. Gm. Vi ollo

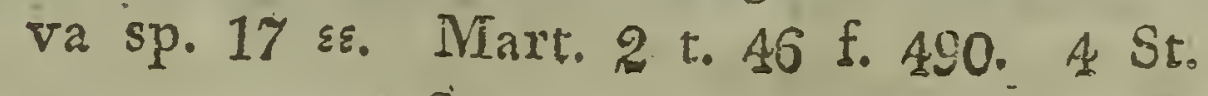

$445 . . \approx \approx-2 s_{0}$ 
446. .39 P. Caesia. Die graublaue Dattel. Gm. Vol. hiatula sp. $20 \%$ Mart. 2 tab. 50 fig. 555. Lister tab. 729 fig. 17. 2 St.

447...40 P. Staphylea. Die gelbe Dattel. Gm. V. oliva sp. 17 a. Chemn. 10 tab. 147 fig. 1381. Lister tab. 729 fig. 17, 18. 2 St.

448...41 P. Cornea. Die hornfarbene Dattel. 1 St.

449...42 P. Umbrosa. Die Licht - und Schatten-Dattel. Gm. Voluta ispidula \& sp. 25. Mart. 2 tab. 49 ,fig. $53 \%$. Knorr 1 tab. 15 fig. 7. 3 St.

\section{Porphyria. Die Dattel.}

1. Clavicula elevata. Mit hervortretender Spindel. 450...43 P. Arachnoidea. Die Spinneweben-Dattel. Gm. V. oliva sp. 17. Mart. 2 tab. 48 fig. 509, 10. 1 St.

$451 \ldots-\infty-2 \mathrm{St}$.

452...44 P. Litterata. Die Buchstaben-Dattel. Gm. sp. 17.

V. Oliva $\gamma \%$. Mart. 2 t. 46 f. 488.14 St.

453...45 P. Fulgurator. Die Blitz-Dattel. Gm. sp. 17 V.

Lade 21.

454...45 P. Utriculus. Die Schlauch - Dattel. Gm. Voluta sp. 19. Mart. 2 tab. 49 s. 51 fig. $539-566$. a. Knorr V. 2 tab. 12 fig. 4, 5. \%. Knorr Vergn. 5 tab. 4 fig. 4. 27 Stück (wovon 2 Stück vorzüglich schön.)

$455 \ldots 45$ * P. Tumida. Die aufgeblasene Dattel. Lister tab. 746 fig. 40. 1 St.

456...A7 P. Texilis. Die gewebte Dattel. 1 St.

2. Clavicula sub-depressa. Mit etwas eingedrückter Spindel.

457 ..48 P. Urceus. Der Krug. Gm. V. oliva sp. 17 «. Mart. 10 tab. 147 f. 1367. Kämmerer pag. 65 tab. 4 fig. 1, 2. $5 \mathrm{St}$.

459...50 P. Bulbosa. Die knolligte Dattel. Gm. sp. 17 oliva. Mart. 2 tab. 47 fig. 507, 508. 2 St.

450...51 P. Tulberosa. Die kanehlfarbene Dattel. Kärameror tab. 3 fig. 7,8 . 3 St.

451...52 P. Ziczac. 1)ie Ziczach-Dattel. 4 St. 
462...53 P. Coffea. Die Kaffeebolne. Gm. sp. 24 V. carneolus. Mart. 2 t. 46 f. 495.4 St.

\section{Cucullus. Die Tute. \\ * Pyriformes. Birnfürmige. \\ 1. Coronata. Gekrönte.}

163...1 C. Imperialis. Die Kaiserkrone. Gm. sp. 2. Mart. Theil II. tab. 62 690-92. Knorr Vergn. Th. II. tab. $11 \mathrm{fig}$. 2. $14 \mathrm{St}$.

464...2 * C. Corona-Ducalis. Die Herzogs-Kronẹ. Gmel. Conus imperialis sp. $2 \%$. Mart. 2 t. 62 f. 693. 1 St. 465...2 C. Regius. Die Königskrone. Gm. sp. $2 \%$. Mart, 10 tab. 139 fig. 1289. 3 St.

466...3 C. Corona Civica. Die Bürgerkrone. Mart. 2 tab: 61 fig. 678. 1 St.

467...4 C. Marmoreus. Die Herz-Tute. Gm. sp. 1. Marto Th. II. tab. 62 fig. 685. 19 St.

168...- - - Mart. 2 t. 62 f. 686. $z$ St.

\section{Lade 22.}

$469 . . .-\beta-2$ St.

470...5 C. Proarchitalassus. Der Schout by Nagt. Gm. sp. 1 C. marmoreus, $\alpha$. Mart. 2 tab. 62 fig. 685. $\beta$. Thnorr 1 tab. 7 fig. 4. 12 St.

$471 \ldots-\alpha-2$ St.

$472 \ldots-\beta-3$ St.

$473 \ldots-\gamma-\gamma-1$ St.

474...6 C. Equestris. Der gebandete Schout by Nagt. Gm.

C. marmoreus sp. $1 \%$ Chemn. 10 t.193 f. 1279. 2 St.

475...7 C. Torquatus. Der Fitter. Gm. C. marmoreus sp.

$1 \%$. Chemn. 10 tab. 138 fig. 1279. 1 St.

$476 \ldots-\alpha-2$ St.

477...8 C. Turritus. Das Mauerwerk. Gm. C. coronatus sp.

39 8. Chemn. 10 tab. 139 fig. 1286-83. 3 St.

478...9 C. Coronalis. Die Kranz-Tute. Gm. sp. 39. 1 St. -... $\alpha-\alpha$ - 1 St.

479...10 C. Araneosus. Die Netz-Tute. Gn. C. masmoreus sp. 1 . . Chemn. 10 tab. 139 fig. 1292.

480...11 C. Arachnoideus. Die Spinnweben-Tute. Gm. sp. 31 Knorr 6 t. 4 f. 4 . Mart. 2 t. 61 f. 676.4 St. 
481...12 C. Trapezium. Die Würfel-Tute. Lister 773 /fig. 19. 1 Stück.

182...13 C. Spurius. Der Bastart-Admiral. Knorr 6 tab. 1 fig. 2. 26 St.

*483..14 ! C. Geographicus. Die Eylands.Tute. Gm. C. insularis sp. 38. I Stïck, wovon cins vorzüglich schün und selten.

483...- $\beta$ - Mart. 2 t. 62 f. 683. 8 St.

* 485.15 !! C. Petreus. Der Stein-Admiral. Gm. C. amiralis coronatus sp. 10 b. 2 St: vorzüglich schön u. selten. 486...- - - Mart. 2 Vign. 26 p. 214 f. 1. 2 St. 487...- $\beta$ - Mart. 2 t. 62 f. 682. \& St. $488 \ldots-\gamma-$ Knorr 5 tab. 25 fig. 3. 11 St. 489...15* C. Biliosus. Die braungelbe Tute. Gm. C. stercus muscarum sp. $25 \delta$. Chemm. 10 t. 139. f. 1294. 1 St. 490...16 C. Monachus. Der Mönch. Gm. C. rusticus sp. 18 ß. Mart. 2 t. 63 f. 694 . Thnorr 4 t. 13 f. 3. 12 St. 491...17 C. Menzingeus. Die blutige Tute. Gm. C. zeylanicus sp. 41. Chemn: 10 t. 142 f. 1518. 5 St.

492...18 C. Fernambucinus. Die bunte Holz-Tute. MIart. 2 tab. $57 \mathrm{fig}, 632.4$ St.

493...19 C. Virgo. Die Jungfer. Gm. C. sp. 5 4. Ma:t. 2 t. 63 f. 701-3. Knorr \& t. 16 f. 5. 5 St.

\section{Lade 23.}

494...20 C. Arenosus. Die sandige Trutc. Gmel. C. sp. 23. Mart. 2 tab. 63 fig. 695.2 St.

495...21 C. Stercus-muscartum. Der Fliegendreck. Gm. C. sp. 23 \%. Mart. 2 t. 63 f. 69\%. Piumpf. t. 33 f. AA. 10 St. 496...22 ! C. Granulatus. Die granulirte Tute. Gm. C. sp. 52. 1 St.

497...23 C. Punctulatus. Der Mückendrecti. Gm. C. sp. 23 ß C. Stercus-muscarum. Mart. 2 t. 63 f. 698, $992 \mathrm{St}$. 498...24 C. Granulosus. Dic Gänsehant.Tute. Gm. sp. $24 \beta$ C. varius. Mart. 10 tab. 138 fig. 1284. 9 St.

409...24* C. Annularis. Die geringelte Tute. Gm. sp. 24 C. varius. 1 St.

500...25 C. Radula. Die Reibe. Gm. sp. 24 G. varius. 2 St. $501 \ldots 26$ C. Canaliculatus. Die gerillte Tute. 5 St. 502...27 C. Lanidoideus. Die Vinkel-Tute. 2 St. 503...28 C. Folliculus. Die Schote. 1 St. 


\section{Simplices. Ungekrönte.}

(1) 4...29 C. Betulinus. Die Butterwecks-Tute. Gm. C. sp. 20. Mart. 2 t. 60 f. 665 . Knorr 2 tab. 11 f. 3. 11 St. eine von ausserordentlicher Grösse.

505...30 C. Tigris. Die Tieger-Katze. Gmel. C. sp. 20 ß. 2 Stück.

506...81 C. Fraxineus. Die graublaue Tute. Gmel. sp. 15. Chemn. 10 t. 138 f. 1277, 78. I St.

507...32 C. Glaucus. Die cipersche Katze. Gmel. C. sp. 15. Chemn. 10 t. 138 f. 1277, 79. 2 St. $508 \ldots-\alpha-1$ St.

509...33 C. Pavimentum. Der italienische Estrich. Gm. sp. 5 . C. Virgo. Mart. 2. t. 59 fig. $658,51$. Knorr 2. t. 12 fig. 3. $12 \mathrm{St}$.

510...34 C. Lacteus. Die milchweisse Tute. Gm. sp. 15. C. glaucus. Mart. 2 t. $61 \mathrm{fig}$. 673. 5 St.

511...35 C. Quadratus. Die Rauten-Tute. Gm. sp. 2\% C. leoninus. Chemn. 10 t. 140 f. 1300.1 St.

512...35 C. Quadratulus. Die Blumen-Tute. Gm. sp. 15. C. glaucus. IMart. 2 t. 61 fig. 670. 71. 6 St.

\section{Lade 24.}

513...37 C. Syriacus. Die syrische Schrift. Gmel. sp. 67. C. spurius. Mart. 2 t. 56 f. 627 . Knorr 3 t. 18 f. 5 t. 9 f. 6. t. 22 f. 3. 9 St.

$514 \ldots-\alpha-$ - - Knorr S tab. 26 fig. 1. 8 St.

515...38 C. Ferugineus. Die rostige Tute. Gm. sp. 6\% C. spu. rius var. Mart. 2 t. 56 fig. $626-28.2$ St.

$516 \ldots . . .99$ C. Litteratus. Die Buchstaben-Tute. Gmel, sp. 3 Mart. 2 tab. 60 fig. 66s; 23 St.

517... - $\alpha$ - - Knorr 1 t. 16 fig. 3. 2 St.

518...40 C: Byssinus. Die arabische Tute. Gm. sp. 3. C. lite teratus $\beta$. MIart. 2 t. 60 fig. 669 . \& St.

51.9...41 C. Pardus. Der Parder. Gmel. sp. 3. C. litteratus, Mart. 2 t. 60 fig. 667 . Knorr 1 t. 16 f. 3. $3 \mathrm{St}$.

$520 . . .42$ C. Leopardus. Der Leopard. Gm. sp. 3. C. litteras tns. Mart. 2 t. 60 f. $666.5 \mathrm{St}$. 


\section{Lade 25.}

5:1...49 C. Sphinx. Die chinesische Bastard-Tute. Gmel. C. genuanus sp. 14. Mart. 2 t. 56 f. 624, 25. Knorr 3 t. 1 f. 1.13 St.

522...44 ! ! C. Papilio. Der Papillions-Flügel. Gm. C. genuanus Papilio, sp. 14. $\beta$. Mart. 2 t. 56 f. 623. Argenv. $t, 12 \mathrm{fig} . \mathrm{V}, 3 \mathrm{St}$.

*523..45 ! ! C. Genzuinus. Die ächte chinesische Tute. Gm. sp; 68. C. vexillum. Lister 763 f. 12. ß. Chemn. 10 t. 144. A. f. m. n. die Flagge der dreizehn vereinigten Staaten. 1 St.

524...46 C. Ebraeus. Die Bauern-Musik. Gm. sp.22. Mart.2 t. 56 f. 617 . Knorr 5 t. 6 fig. 2. 11 St. i

-...- $\alpha-$ - 3 St.

525...47 C. Chaldaeus. Die schwarze Tute. Gn. C. princeps, sp. 9. Mart. 2 t. 63 f. 699, 700. Knorr 3 tab. 4 f. 2. 5 St.

525...48.C. Figulinus. Die Eichenholz-Tute. Gmel. sp. 21. Knort 5 tab. 25 fig. 2. 4 St.

$527 \ldots-\alpha=-\boldsymbol{7}$ St.

$529 . . .-\beta_{1}:-3 S t$.

*529..-! $y-1$ St. (knolligt.)

530...49 C. Buxeus. Die Buxbaumholz-Tute. Gm. sp. 21. Knorr 3 t. 11 fig. 2. Mart. 2 t. 59 fig. 656, 58. 5 St. 531...50 C. Cardinalis. Die Cardinals-Tute. Gm. C.aurifiacus, sp. 55. Chemn. 10. t. 144. A. f. 1. 2 St.

532...51 C. Virgo. Die Menonisten-Tute. Gm. sp.5. Mart.2. t. 53 fig. 595 . Knorr 2 t. 24 fig. $4 \& 3$ t. 22 f. 1. 6 St. 533...52 C. Gualterianus. Die gualterische Tute. C. spurius Gm. sp. 67. Gualt. 21 fig. F. 1 St.

534...53 C. Cinamomens. Die hanelilfarbene Tute. Gmel. C. capitaneus, sp. 6. Mart. 2 t. 57 f. 680, 81.13 St.

$-\ldots-\alpha--1 S t$

535...54 C. Canonicus. Der Domlerren-Mantel. Gmel. C. vexillum, sp. 68 . Mart. 2 t. 57 fig. 629. Knorr 3 t. 1 fig. 3. 4 St.

533j...55 C. Miles. Der Soldat. Gm. sp. 8. Mart. 2 tab. 59 fig: 663,6 . Knorr 1 t. 15 fig. $4 \& 3$ t. 1 fig. 3.12 St: 


\section{Lade 26.}

537...56 C. Capitaneus. Der Hauptmann. Gm. sp.6. Mart. 2 t. 59 fig. 660 -62. Knorr 1 t. 15 fig. 3. 9 St.

$538 \ldots-\alpha-5 \mathrm{St}$.

$539 . . .-\beta-2 \mathrm{St}$.

$540 \ldots-\gamma-1$ St.

$541 \ldots-\delta-5 \mathrm{St}$.

542...- - - - Chemn. 10 t. 138 fig. 1280. 5 St.

$543 \ldots-\zeta--1$ St.

$544 \ldots-\eta-2$ St.

545...57 ! C. Archithalassus Indiae. Der westindische Admiral. Gm. C. Thomae, sp. 70. Chemn. 10 t. 138 fig. 1282, 83. $10 \mathrm{St}$.

545...58 C. Medusae. Der Medusenkopf. Gm. C. betulinus. medusa, sp. 20. $\beta$. Mart. 2 t. 61 f. 675 . Chemn. 10 t. 142 fo 1321. 1 st.

547...59 C. Achatinus. Die Agat-Tute. Gm. C. leoninus, sp. 27 ß. Mart. 2 tab. 55 fig. 605, 605. 13 St.

548...59* C. Barathrum. Die weitmündige Tute. Gmel. C. leoninus, sp. 2\%. Mart. 2 t. 55 f. $60 \%$. 1 St.

549...60 C. Theobroma. Die zerrissene Netz-Tute. Gm. C. zeylanicus, sp. 41 . Chemn. 10 t. 142 f. 1318. 2 St.

\section{Lade. 27.}

550...61 C. Flammeus. Die flammigte Tute: Gm. sp. 27 C. leoninus. Mart. 2 t. 55 f. 605, 607. 1 St.

551 ...62 C. Leoninus. Die klimmende Löwentute. Gm. sp. 10 Ammiralis americanus. Mart. 2 t. 57 f. 640. 2 St. 552...63 C. Isabella. Die isabelfarbene Tute. I St:

553...63* C. Bifasciatus. Die zweibandige Tute. 1 St. *554..64 ! C. Cèdo Nulli. Die Cedo-Nulli-Tute. 3 St. 555...65 C. Annulas. Die Ring-Tute. 1 St.

555...66 C. Pulverulentus. Die gestippelte Tute. Gm. sp̃. 57 C. magus. Nart. tab. 58 fig. 641 . Knorr 6 tab. 16 fig. 5 \& tab. 1 fig. 3. A St.

557...67 C. Cereolus. Die Wachskerze. Gm. sp. 4 C. generalis, $\beta$. Chemn. 10 tab. 140 fig. 1301. 1 St.

558...68 C. Orleanus. Die orange Tute. Gm. sp. 6 C. capi。 taneus, d. Mart. 10 tab. 144 fig. 1298. Knorr 3 tab. 18 fig. 3. 6 St. 
559...69 C. Filosus. Die umsponnene Tute. Favanne $15 \mathrm{fig}$. C. 1 St.

560... \% C. Ornatus. Die geschmïchte Tute. Gm. sp. 4 C. generalis, $\beta$. Mart. 10 tab. 140 f. 1301-S. 4 St.

561...71 C. Dux. Der Feldmarschall. Gm. sp. 4 C. gereralis. Mart. 2 tab. 45 fig. 618. I St.

562...72 C. Generalis. Der General. Gm. sp. 4. Mart. 2 t. 58 f. 646. Knorr 1 tab. 7 fig. 3. 2. tab. 5 fig. 2 \& 3 , ti.b. 17 fig. 4,5 \& tab. 18 fig. 3, 4. 11 St.

563...- - - - Mart. 2 t. 58 f. $649-52.14$ St.

564...73 C. Locumitenens. Der Lieutenant. Gm. sp. 4 C. ge, neralis. Mart. 2 tab. 58 fig. 645 . 2 St.

565...74 C. Eburneus. Die elfenbeinerne 'Tute. Gm. sp. 48 C. ochtoleucus. Mart. 2 t. 52 f. 573. 2 St.

566...75 C. Amadis. Die Amadis-Tute. Gm. sp. 32. Chemn. 10 t. 142 f. 1322. Knorr 6 t. 5 f. 3. 9 St.

567...76 C. Vicarius. Der Vice-Admiral. Gm. sp. 10.Ammiralus Americanus b. Mart. 2 tab. 57 fig. 638, 39. Knorr 5 táb. 24 fig. 4: 5 St.

$568 \ldots-\infty-2$ St.

569...77 C. St. Thomae. Die St. Thomas-Tute. Gm. sp. 70. Chemn. 10 tab. 138 fig. 1981 n. 2.'Mart. 2 tab. 53 fig. 590. 2 St.

570...78 C. Pulchellus. Die schöne Tute. Favanne tab. 79 fig. L. 2 St.

*571..79 ! C. Ammiralis. Die Admirals-Tute. Gm. sp. 10 :C. occidentalis. Mart. 2 tab. 57 fig. 635. I St.

*572.. - ! «. C. Summus. Der Ober-Admiral mit 3 Bänden. Gm. sp. 11 C. Vicarius. Chemn. 10 tab. 141 fig. 1307. 4 St.

*573..- - ß. C. Princeps. Der Fang-Admiral, mit 4 Banden, Gm. C. ammiralis summus, a. sp. 10. Favanne t. 17 f. S. Knorr 1 tab. 8 fig. 2. 2 St.

*574..- ! \& C. Coronatus. Der gekrönte Admiral, mit 5 Bänden. Gm. sp. 10. Chemu. 10 t. 141 f. 1308, 9. 2 St.

*575..-! $\delta *$ C. Imperialis. Der kaiserliche Admiral, gelblich mit 4 Bänden. 1 St.

*576.. - ! r. C. Granulatus, Der granulirte Admiral, nit Bänden. 1 St.

*577..- ! \&. C. Torquatus. Der extra Admiral, mit 6 Bändex. Favanne tab. $17 \mathrm{fig}$. S. 4. $2 \mathrm{St}$. 
578... - ! 3. C. Equestris. Der Pitter, mit 5 Bănden. Favanne tab. 17 fig. S. 2. 1 St.

579...-- ! C. Larvatus. Der verlarvte Admiral, mit vier schmalen Bänden. Gm. sp: 10 \%. Mart. 2 tab. 57 fig. 635 a. Favanne tab. 17 fig. S. 3. 1 St.

580...- ท. C. Dubitatus. Der After-Admiral. 1 St.

$581 \ldots 80$ C. Crenulatus. Die gerillte Tute. $2 \mathrm{St}$. 582...81 Tornatus. Die Gedrechselte Tute. 2 St.

583...82 C. Minutus. Die kleine Tute. Gm. sp. 16 C. mo: nachus. Mart. 2 tab. 55 fig. 612 a. 8 St.

584...83 C. Nubecula. Die gewölkte Tute. Gmel. sp. 66. Mart. 2 tab. 56 fig. 622.7 St.

585...84 C. Parvus. Die kleine Tute. Gm. sp. 66. 1 St. 586...85 C. Nubilus. Die durkle Tute. Gm. sp. 27 C. leoninus, $\delta$. Mart. 2 tab. 55 fig. 610.6 St.

587...86 C. Pellucidus. Die durchsichtige Tute. 2 St.

588...87 C. Clavatus. Die aschgraue Tute. Gm. sp. 18 C. rusticus; ж. Mart. 2 tab. 52 fig. 5\%8. 2 St.

\section{Lade 28.}

589...88 C. Cinereus. Der Aschen-Püster. Gm. sp. 18 C. rus。 ticus. Mart. 2 tab. 52 fig. 578 . 4 St.

590...89 C. Cinerarius. Der Aschentopf, Knorr 3 tab. 16 fig. 3. grawe Monnik. 1 St.

591...90 C. Spectrunt. Das Gespenst. Gm. sp. 62. Mart. 2 tab. 53 fig. 581-83. Knorr 2 t. 8 f. 4. 9 St. $592 \ldots-\alpha-4 \mathrm{St}$.

593...91 C. Lineátus. Die liniirte Tute. Gm. sp. 62 C. spec. trum. Chemn. 10 tab. 140 fig. 1304. Knorr 2 tab. 8 fig. 4. I St.;

$594 ., .91$ * C. Albeolus. Die wieisse Kerze. Gm. sp. 44 C. terebellum. Mart. 2 t. 52 fig. $5 \% 7.1 \mathrm{St}$.

595...92 C. Chinensis. Die chinesische Schrift. Gmel. sp. 62 C. spectrum, $\gamma$. Chemn. 10 t. 144 a. f. g. l. 3 St. 596...92* C. Antillarum. Der englische Admiral. Gm. sp. 47 C. lactus: Knorr 3 tab. 6 fig. 5. 7 St.

597...99 C. Carota. Die rothe Rübe. Gm. sp. 62 C. spectrum. Mart. 2 tab. 52 fig. 581. 1 St.

*598..93 * !! C. Aurifiacus. Der Orange-Admiral. Gmel. sp. 56. Knorr 5 tab. 24 fig. 1. 1 St. 
*gG9..94! C. Venustus. Die liebliche Tute. 1 Stück. Eine höchstseltne weisse dünnschaligte mit blassrothen Wolken bezeichnete Tute, zum Geschlecht der Amadis gehörend, doch etwas bäuchigt.

$600 \ldots 95$ C. Danccus. Die gelbe Rübe, orange farbigt. 1 St. $601 \ldots 96$ C. Sponsa. Die Braut. 3 St.

602...96* C. Purpureus. Die blutrothe Tute. Gm. sp. 64. C. tulipa B. 1 St.

603...97 C. Mercator. Der Kaufmann. Gm. sp. 19. Mart. 2 tab. 56 fig. 620 . Knorr 2 tab. 1 fig. 4. 5 St.

604...98 C. Aurelius. Das goldne Netz. Gm. sp. 19. Mart. 2 tab. 55 fig. 621 . Knorr 2 tab. 1 fig. 4. I St.

605...99 C. Mille punctatus. Die tausendfach punctirte Tute. Gm, sp. 36, C. leucostictus. Chemn. 10 tab. 140 fig. 1305. $6 \mathrm{St}$.

606...100 C. Caffer. Die zimmetbraune Tute. Gm. sp. 31 C. cofféae. Mart. 2 tab. 56 fig. 618. 2 St.

60\%...101 C. Anonymus. Die namenlose Tute. 2 St.

$608 . .102$ C. Novem striatus. Die neunmal gestreifte Tute. Gm. sp. 33 C. fulmineus. Mart. 2 t. 58 f. 644.2 St. 609...103 C. Tornatus. Die ausgedrechselte Tute. 1 St.

610...104 C. Jaspideus. Die körnigte Tute. Gmel. sp. 28. Mart. 2 tab. 55 fig. 612. 1 St.

611...105 C. Circumductus. Die weisse Schout. 2 St. $612 \ldots 105 *$ C. Politus. Die geglättete Tute. 1 St.

613...106 C. Circumvolutus. Die graugrüne Tute. 1 St.

614...107 C. Saphirus. Der Saphir. 1 St.

615...108 C. Glaucus. Die, bläuliche Tute. Gm. sp. 17 C. minimus. Mart. 2 tab. 55 fig. 613. 3 St.

616...109 C. Caesius. Die weissblaue Tute. Gm. sp. 25 C. achatinus. Mart. 2 tab. 52 fig. 580. 1 St.

617...110 C. Crucifer. Die Khreutzträgerin. Gm. sp. 27 C. leoninus, $\beta$. Mart. 2 t. 55 f. 605.2 St.

618...110 * C. Camelinus. Die kanehlfarbene Tute. 1 St.

619...111 C. Cutis anguina. Die Schlangenhaut. Gm. sp. 27 C. leoninus. Mart. 2 tab. 55 fig. 605 .

620..112 C. IMaculosus., Die bunte Tute. Gm. sp. 29 C. nebulosus, $\beta$. Ýnorr 5 tab. 18 fig. 4 . Knorr 3 tab. 16 fig. 2. $8 \mathrm{St}$.

621...113 C. Guttatus. Die getropfelte Tute. Gm. sp. 29 C. nebulosus, $\beta$. Mart. 2 t. 55 f. $613,14,1 \mathrm{St}_{\text {, }}$ 
629...114 C. Ventricosus. Die bauchigte Tute. Gm. sp. 25 C. achatinus. Chemn. 10 t. 142 f. 1317. 2 St.

\section{Cucullus, Die Tute.}

* * Cylindraceae. Walzenförmige.

\section{Coarctatoe.}

624...115 C. Magus. Der Zauberer. Gm. sp. 57. Mart. 2 tab. 58 fig. 641 . 25 St.

$625 \ldots-\alpha-1$ - St.

$626 \ldots-\beta-1$ St.

627...116 C. Arenatus. Die Staub-Tute. Gm. sp. 23 C. ster. cus muscarurn. Mart. 2 tab. 64 fig. 711, 12.9 St. 628...116 * C. Sabella. Die rothe Sandi-Tute. Gm. sp. 23 C. stercus muscatum. Mart. 2 t. 64 f. 713. 3 St.

629...117 C. Nobilis. Die bunte Madam. Gm. sp: 13. Mart. 1 tab. 62 fig. 689. Chemn. 10 t. 141 f. 1312. 4 St.

630...118 C. Aureus. Das goldne Zeug. Gm. sp. 60 C. auricus. 1 St.

$631 \ldots$ - $\propto$ - Gm. sp. 60 \&. Mart. 1 t. 54 f. 601. 2St 632...119 C. Auriges. Die verbrehmte Tute. Gm. sp. 59 C. textile. Mart. 2 tab. 54 fig. 599. 6 St.

\section{Lade 29.}

633...120 C. Gloria maris. Die Gloria Maris-Tute, 2te Sorte. Gm. sp. 59 C. 'textile. Mart. 2 tab. 54 fig. 598, 600, $602 \beta$. Chemn. 10 tab. 1326 a. 22 St.

634...121 C. Aurifer. Die vergoldete Tute. Gm. sp. 60 C. aulicus. Knori 2 tab. 1 fig. $1-3$ \& 3 tab. 19 fig. 1. $19 \mathrm{St}$.

635...122 C. Auratus. Die güldische Tute. Gm. sp. 59 C. textile. Knorr 2 tab. 8 f. 3 \& 3 t. 18 f. 2.7 St.

636...123 C. Textile. Die Goldlaks-Tnte. Gm. sp. 59. Mart. 2 tab. 54 fig. 598. 10 St.

657... - a - - Chemn. 10 tab. 143 fig. 1326. 2 St.

638...124 C. Aulicus. Die bunte Tute. Gm. sp. 60. Mart. 2 tab. 5 fig. 592, 595. Knorr a tab. 19 fig. 1. 13 St. 639...125 C. Gentilis. Die kurzgespindelte Brunet-Tute. Gm.

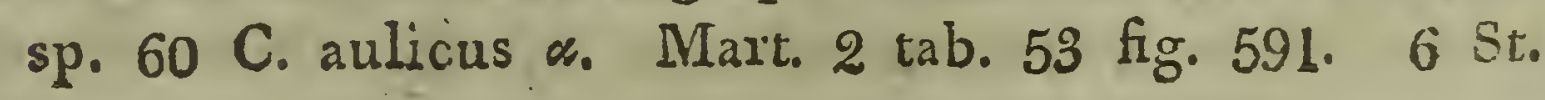




\section{Lade 30.}

640...126 C. Striatus. Das Wolkhorn. Gm. sp. 58. Mart. 2 tab. 64 fig. 714-16. Knorr 1 tab. 18 fig. 1 \& 8 tab. 12 fig. 5 tab. 21 fig. 1.18 St.

641...127 C. Nussatella. Die Sand.Tute. Gm. sp. 43. Mart. 2 tab. 51 f. 567 . Knorr 2 t. 4 f. 7.9 St.

612...128 C. Terebellum. Der Küperbohrer. Gm. sp. 49 C. laevis. Mart. 2 t. 52 f. $5720^{\circ} 1 \mathrm{St}$.

643...129. C. Candidus. Der weisse Küperbohrer. I St.

*644..130! C. Purpuratus. Der vice Orange-Admiral. Gm. sp. 50 C. affinis. Mart, 2 tab. 57 fig. 571.1 St.

645...131 C. Chrysalis. Der gelbe Küperbohrer. 1 St.

\section{Laxa.}

646...132 C. Geographus. Die Agat-Bake. Gm.sp. 65. Mart, 2 tab. 64 fig. 717. Knorr 3 tab. 21 f. 2. 10 St.

647...133 C. Tulipa. Die Tulpe. Gm. sp. 64. Mart. 2 tab. 64,65 718, 721. Knorr 3 tab. 11 fig. 4 \& 5, tab. 20 fig. 1, 2. 11. St.

648...134 C. Laganum. Die Purpur-Bake. Gm. sp. 63. C. bullatus. Knorr 5 tab. 11 fig. A. 1 St.

649...135 C. Bullatus. Die orange Bake. Gm. sp. 63. Ch. 10 tab. 142 fig. 1315, 16. 2 St.

650...136 C. Capitatus, Der Stockknopf. 1 St,

\section{Lade 31.}

\section{Pterygia. Halbe Flügel-Schnecke.}

651...1 P. Glabella. Die glatte halbe Flügel-Scknecke. Gm. sp. 32 Voluta Glabella. Mart. 2 t. 42 f. 429. Knorr 4 tab. 21 fig. 2, 3. 4 St.

652...2 P. Nebulosá. Die wolligte halbe Flügel-Sclınecke.

Gm. sp. 32. Mart. 434, 435. $10 \mathrm{St}$. $653 . . . \infty-\infty$ St.

654...2 P. Vulgaris. Die kleine gemeine h. Flügel-Sohnecke.

Gm. sp. 35 Voluta mercatoria. Mart. 2 tab. 44 fig. $455,57.2 \mathrm{St}$.

$655, \ldots 2 *$ P. Amoena. Die hübsche halbe Tlügel-Schnecke.

Gm. Voluta mercatoria, sp. 35. Mart. 2 tab. 44 fig. 458. 2 St. 
56...3 P. Erminea. Die Hermelin halbe Flügel-Schnecke.

Gm. Voluta faba, sp. 31. Mart. 2 tab. 42 fig. $432,33$.

Knorr 4 t. 17 f. 6.2 St.

$657 . . .3 *$ P. Subterranea. Die unterirdische halbe Flügel-

Schnecke. Favanne tab. 56 fig. J. 1. Mart. 4 tab. 142

fig. 1330. 3 St.

$658 \ldots 3^{* *}$ P. Effossa. Die ausgegrabene halbe Flügel-Schnecke.

Fav. t. 56 f. J. 1. Mart. 4 t. 142 f. 1330. 4 St.

659...4 P. Pruna. Die Pflaume. Gm. sp, 33 Voluta prunum.

Mart. 2 t. 42 f. $422,23.5 \mathrm{St}$.

660...5 P. Persicula. Die gesäumte halbe Flügel-S.chnecke.

Gm. sp. 29 Voluta persicula, $\%$. Mart. 2 t. 42 f. 416.

2 Stück.

661...6 P. Crassa. Die doppelt gesäumte h. Flügel-Schnecke.

Gm. sp. 29 Voluta persicula, «. Mart. 2 t. 42 f. 421.

Chemn. 10 t. 150 f., 1421. 1 St.

662...7 P. Fossilis, Die Fossil halbe Flügel-Schnecke. 8 St. 663...7* P. Turris. Die langgestreckte h. Flügel-Sclinecke.

1 Stück.

664...8 P. Magellanica. Die magellanische h. Fligel-Schnecke. Gm. Voluta spectabilis, sp. 142. Chemn. 10 tab. 148 fig. 1383, 84. Kämmerer 7 fig. 1. Knorr 4 tab, 29 fig. 1, 2. 1 St.

665...9 P. Peregrina. Die gelbe magellanische halbe FlügelSchnecke. Gm. Voluta spectabilis. sp. 142. Chemn. 10 t, 148 f. 1383, 84. Knorr. 4 t. 29 f. 1, 2. 2 St.

*665a.9 ג. !! P. Fulgura. Die Blitzstrahlen h. Flügel-Schnecke. Gm. sp. 106 Voluta rupestris. Mart. 3 tab. 98 fig. 941, 42. $1 \mathrm{St}$.

666...10 P. Ancilla. Die geflammte magellanische halbe Flügel-Schnecke. Gm.sp. 110 Voluta magelanica. Chemn. Tom. X. t. 148 f. 1383, 84. Knorr 5 t. 23 f. 2. 1 St. 667...11 P. Volva. Die weisse halbe Flügel-Schnecke. Gm. sp. 126 V.ol: Chemn, 10 t. 148 f. 1389, 90. 2.St. 668...12 P. Edentila. Die zahnlose h. Flügel-Schnecke. $6 \mathrm{St}$ 669...13 P. Nucella. Die INuss. Gm. sp. $25 \mathrm{~V}$. dactylus. Iister tab. 813 fig. 23. Chemn. 10 tab. 150 fig. 1411, 12. 3 St.

670...14 P. Pyriformis. Die unreife Birne. 2 St.

671...15 P. Conoidea. Die braungefleckte Birne. Gm. sp. 140

V. conus. Chemn. 10 to 150 f. 1415,16 . 2 St. 


\section{Morum. Die Maulbeere.}

672...1 M. Purpureum. Die purpurfarbene Maulbeere. Gm. sp. 18 Strombus oiscus. Mart. 2 t.ab. 34 fig. 357, 58. Knorr 4 tab. 12 fig. 4. 6 St.

\section{Galeodes. Der Helm.}

673...1 G. Melongena. Das Bettzeug. Gm. Murex Melon• gena, sp. 50.

$674 . . .-\alpha-$ - Mart. 2 t. 39 f. $393,397$. St.

$675 \ldots-\beta-3$ St.

$676 \ldots-\gamma-$ Knorr 1 t. 17 f. $5.5 \mathrm{St}$.

$677 \ldots-\delta$ - Mart. 2 t. 39 f. 391. 7 St.

678... - - - Chemn. 10 t. 164 f. 1568. 7 St.

\section{Lade 32.}

679...2 G. Tuberosa. Der linolligte Helm. Gm. Murex hip-

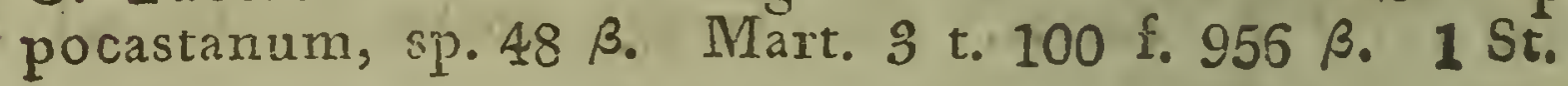

$680 \ldots 3$ G. Mespilas. Die Mispel. 2 St.

\section{Thais. Thais.}

1. Clavicula depressa. Mit kurzer Spindel.

681...1 T. Lena. Die Kuplerin. Gm.'sp. 44 Murex fucus. Mart. 3 t. 100 f. 959, 60. 7 St.

682...2 T. Meretricula. Das Freudenmädchen. Gmel. sp. 44 Murex fucus. Mart. 3 t. 100 f. 961, 62, 1 St.

2. Clavicula elevata. Mit hervortretender Spindel. 633...5 T. Patula. Der Weitemund. Gm. Buccinum patulum sp. 51. Mart. 758, 59. Knorr 6 t. 24 f. 1. 7 St. $684 \ldots 4$ T. Persica. Die Schnürbrust. Gm. Bucc. persicum, sp. 49. Mart. 3 t. 69 f. 760.6 St.

685...5 T. Panama. Die Panama. Gm. Buccin. haemośtoma, sp. 52. Chemn. 10 t. 154 f. $146 \%, 68.5 \mathrm{St}$.

686...6 T, Grisea. Der blasgraue Weitemund. Gm. Buccin. haemostoma sp. 52. Mart. 3 t. 101 f. 964. 2 St.

687...7 T. Metallica. Das Metall-Horn. Gm. Bucc. haemostoma, sp. 52. Mart. 3 tab. 101 f, 965, 66. 7 St.

$683 . . .-\infty-2$ St.

$689 . . .-\beta-1 \mathrm{St}$.

$690 \ldots-\gamma-1$ St. 
691...8 T. Stellata. Das gezakte Metall-Horn. Gm. Buccin. haemestoma.sp. 52. Argenv. t. 17 f. H. 1 St.

692...9 T. Nebulosa. Das dunkle Metall-Horn. Gm. Buccin. haemostoma sp. 52. 1 St.

693...10 ! T. Monoceros. Das Einhorn. Gm. Buccin. monodon, sp. 50. Mart. 3 t. 69 fig. 761. Chemn. 10 t. 151 f. 1469, 70. Knorr 4 t. 30 fig. 1. $2 \mathrm{St}$.

\section{Drupa. Die unreife Olive.}

1. Columella depressa. Mit eingedruckter Spindel. 694...1 D. Morum. Die Maulbeere. Gm. Mutex neritoideus, sp. 43. Mart. 3 t. 101 f. 972, 978. Knorr 1 t. 25 f. $5, \div 6.5 \mathrm{St}$.

695...2 D. Tribulus. Die Wassernuss. Gm. Mutex ricinus, sp. 41. Rumpf t. 24 f. E. Gualt. t. 28 f. N. 2 St. 696...3 D. Pubus caesius. Die Himbeere. Gm. Murex neritoideus, Mart. 3 t. 101 f. 976, 77. 4 St. $697 \ldots-\alpha-3 \cdot S t$

698...4 D. Rubris idaeus. Die weisse Hindbeere (Hohlbeere). Gm. Murex hystrix, sp. 46. Mart. 3 t. 101 f. 974.75 . Knorr 6 t. 24 f. 7.4 St.

699...5 D. Frugum. Die Erdbeere. Gm. Murex nodus, sp. 42. var. 1 St.

700..6 D. Grossularia. Die Stachelbeere. Gm. Murex neritoideus, sp. 43. Mart. 3 t. 102 f. 978, 79. 2 St.

2. Columella elevata. Mit hervortretender Spindèl. 701...7 D. Chamaemoris. Die schwarze Maulbeere. Gmel. Murex mancinella, sp. 47. Lister 954 f. 4. Knom 4 t. 26 f. 2.2 St.

702...7* D. Botroides. Die Weintraube. Favanne t. s3 f. B. $2 \mathrm{St}$.

703...8 ! D. Uva. Die Weinbeere. Gm. Murex mancinella, sp. 47. Mart. 8 t. 101 f. 970. Lister t. 954 f. 5. 1 St. $704 . . .9$ D. Cornus. Die Kaneel-Kirsche. G̈m. Murex mancinella, sp. 47. var. Mart. 3 t. 101 f. 971. 2 St. 706...10* D. Muricina. Die purpurfarbene Maulbeere. Gm. Murex Sacellum, sp. 164. Chemin. 10 t. 163. f. 1561 , 62. $1 \mathrm{St}$. 
707...10** D. Glans. Die Eichel."m Gm. Murex despectur sp. 74. Chemn. io t. 153 f. 1558 . I St.

708...11 D. Mancinella. Die weisse Maulbeere. Gm. Murex mancinella, sp. 47. Knorr 3 t. 29 fig. 6. 4 St.

709...12 D. Trapa. Die grosse Maulbeere. Gm. Murex hip. pocastanum, sp. 48 ß. Mart. 3 t. 100 f. 95\% 5 St.

710...13 D. Aesculus. Die dunkle Maulbeere. Gm. Murex hippocastanum, sp. 43. var. 3 St.

\section{Lade 33.}

\section{Trasum. Die Vase.}

711... V. Urna. Der Aschentopf. Gm. Voluta capitellum, sp. 100 ß. Mart. 3 t. 99 f. 949,50 . Knorr 6 t. 35 f. 1.7 St.

$712 . .2$ V. Capitellum. Die gezackte Schweitzer-Hose. Gmel。 Voluta capitellum, sp. 100. Mart. 94\%, 948. 6 St.

713...3 V. Turbinellus. Der kurze Morgenstern. Gm. Voluta turbinellus, sp. 99. Mart. 3 t. 99 f. 944. Knorr 2 t. 2 f. 3. 14 St.

714...4 V, Ceramicum. Der gestreckte Morgenstern. Gmel. Voluta ceremica, sp. 101. Mart. 3 t. 99 f. 943. Knorr 5 t. 4 f. 2. 7 St.

$715 \ldots 5$ V. Hippocastana. Die wilde Castanie. Gm. Murex hippocastanum, sp. 48. Mart. 3 t. 99 f. 945, 46. 3 St. 716...6 V. Castaneum. Die Castanie. Gm. Murex hippoca. stanum, sp. 48. B. Mart. 956, 958. \& St.

\section{Volema. Die Pfundbirne.}

1. Pyriformes. Birnförmige.

717...1 V. Curtangniona. Die Curtanjon-Birne. Gm. Voluta pyrum, sp. 102. 2 St.

718...2 V. Myristica. Die weisse Muscatnuss. Gm. Murex hippocastanum, sp.48. Mart.2 t. 40 f. 400, 401. Knorr 6 t. 24 f. 2.2 St.

719...3 V. Aromatica. Die grane Mnscatnuss. Gm. Murex hippocastanum, sp. 48. Mart. 2 t. 40 f. 399. 4 St. 720 ... - a - Knorr 6 t. 35 fig. S. 1 St. $721 \ldots 4$ V. Nux-moschata. Die Muscatnuss. Gm. Murex hippocastanum, sp. 48. Mart, 2 t. 40 f. 39s. 6 St. 
722...5 V. Moschatellina. Die kleine Muscatnuss. Gmel.

Murex hippocastanum, sp. 48. 3 St.

723...6 V. Cotonea. Die graue Birne mit braunen Linien. Gm.

Murex ficus, sp. 66. Mart. 3 t. 94 f. 911.5 St.

724...7 V. Pyrum. Die glatte Birne. Gm. Murex ficus, sp.

66 \%. Mart. 3 t. 94 f. 915,915 a. Chemn. 10 t. 163

f. $1564,65.1$ St.

725...8 V. Paradisiaca. Der Paradies-Apfel. Gm. Murex ficus, sp. 66. Mart. 3 t. 94 fig. 909, 10. 2 St.

2. Pomiformes. Aepfelförmige.

726...8 V. Plicata. Das Manschetten-Horn. Gm. Buccinum bezoar, "sp. 91. Mart. 3 t. 68 f. 754, 55. Argenvillo t. 15 f. G. 9 St.

727...10 V. Consul. Der Bürgermeister. Gm. Murex consul, sp. 159. Chemn. 10 t. 160 f. 1516, 1\%. 7 St.

\section{Lade 34.}

728...11 V. Alouina. Das Alouin-Horn. Gm. Murex hippocastanum, sp. 48. Mart. 3 t. 101 f. 967, 68. 6 St. 729...12 V. Glacialis. Die gefrorne Birne. Gm. Murex hip. pocastanum, sp. 48. Mart. 3 t. 101 fig. 967, 68. var. 1 St.

\section{Pyramis, Die Piramide.}

730...1 P. Lucifer. Das Khamehl-Horn. Gm. Strombus Iucia fer. sp. 19. Mart. 3 t. 90 f. 881 . Is St, $731 \ldots-\infty-5 \mathrm{St}$.

732...2 P. Gallica. Die französische Piramide. d'Argenv. \$\$ fig. 10. 6 St.

733...2 * P. Harpa. Die Harfen-Piramide. 1 St. 734...3 P. Striata. Die gestreifte Piramide. Gmel. Murex cochlidum sp. 63. Mart. 3 t. 90 f. 882.5 St.:

795...4 P. Velutata. Die eingerollte Piramide. Knorr 5 tab. 9 fig. 5. 6 St.

736...5 P. Conoidea. Die kegelförmige Piramide. Mart. \& tab. 91 fig. 893.4 St.

73\%...6 P. Crenulata. Die gerillte Piramide, Mart. 8 tab. 90 fig. 883. $3 \mathrm{St}$. 


\section{Plejona. Die Musik-Schnecke.}

1. Columella multiplicata.

739...1 P. Fossilis. Die verkalkte Musik-Schnecke. d'Argenv. 33, fig. 10. 1 St.

739...2 P. Ebraea. Die wilde Musik-Schnecke. Gm. Voluta hebraea, sp. 98. Mart. 3 tab. 96 fig. 924, 25. 4 St.

740...3 P. Lineata. Die 4 liniirte Musik-Schnecke. Gm. Vol. musica, sp. 96. Mart. 3 tab. 96 fig. 928, 29. \& St.

\section{Lade 35.}

741... A I. Musica. Die 5linigte Musik-Schnecke. Gm, Vol. musica, sp. 95. 12 St.

742...5 P. Chorea. Die starke Musik-Schnecke. Gm. Voluta musica, sp. 95. 4 St.

743...6 P. Incarnata. Die fleischfarbene Musik-Schnecke. Gm. Voluta musica, sp. 96. 4 St.

744...7 P. Maculata. Dic grobe Musik-Schnecke. Gm. Vol. musica, sp. 96. 5 St.

745...8 P. Laevigata. Die glatte Musik-Schnecke. Gm. Vol. musica, sp.:96. S St.

746...9 P. Preticulata. Die Netz-Musik-Schnecke. Gm. Vol. musica, sp. 96. 5 St.

7 $47 \ldots 10$ P. Rosea. Die rothe weissgesprenkelte Musik-Schneck• Gm. Voluta musica, sp. 96. 1 St.

748...11. P. Confusa. Die falsche Musik-Schnecke. Gm. Vol. musica, sp. 96. 3 St.

749...12 P. Turbata. Die verwischte Musik-Schnecke. Gm. Vol., musica, sp. 96. \& St.! (1 Durchschnittene.)

*750..13 ! P. Muta. Die grïne Musik-Schnecke. Gm. Vol. musica, sp. 96. Mart. 3 tab. 97 fig. 932, 39. 4 St.

Vorstehende sind alle von vorzüglicher Grösse und Schönheit.

*\%51..14! P. Aurifiaca. Die Orangeflagge. Gm. V.vexillum, sp. 104. Mart. 3 tab. 120 f. 1098. Chemn. 10 vign. 20 fig. B. Knorr 5 t. 1 f. 1.1 St.

752...15! P. Lapponica. Das Laphorn. Gm. Vol. lapponios, sp. 103. Mart. 3 tab. 95 fig. 920, 21. Chemn. 3. tab. 89 fig. 872,79 . Knox 6 tib. 11 fig. 2. 1 St. 


\section{Calumella quadruplicata.}

*753..16 !! P. Persica. Das persische Warzen-Horn oder Bastard-Gondel. Gm. Vol. scapha. Mart. 3 tab. 72 fig. 774-76. 2 St.

754...17 P. Effossa. Die Fledermaus von Courtagnion. Gm. Vol. vespertilio, sp. 97. d'Árgenv. 8 fig. 10. 1 St. 755...18 P. Vospertilio. Die Fledermaus. Gm. Vol, vespertilio. Mart. 3 tab. 98 fig. 93\%. 8 St.

756... $-\alpha-7$ St.

\section{Lade 36.}

757...19 P. Vantpyrus. Der Vampir. Gm. Vol. vespertilio, sp. 79. Mart. 1397, 1398. 6 St.

758...20 P. Cylindracea. Die gestreckte Flede:maus. Gm. V. vespertilio, sp. 9\%. 1 St.

759...21 P. Murina. Die bunte Fledermaus, Gm. V. vespertilio, sp. 97. Mart. 940. $13 \mathrm{St}_{\text {. }}$

$760 \ldots-\alpha-8$ St.

$761 \ldots-\beta-3$ St.

$762 \ldots-y-1$ St.

763...22 P. Suilla. Der Schweinsrüssel. Gm. Vol. respertilio, sp. 9\%. $13 \mathrm{St}$.

764...- - - - Mart. 3 tab. 98 fig. 938, 39. \& $S_{\text {t. }}$

765...22* P. Arachnoidea. Der schäckigte Schweinskopf. Gm. Vol. vespertilio, sp. 97. 2 St.

766...23 ! P. Bergamotta. Die Bergemot-Birn. Gm. V. Alavi. cans, sp. 105. Mart. 3 tab. 95 fig. 922, 23. 1 St.

767...24 P. Variegata. Der buntschäckigte Schweinskopf; die gezackte Bergamot-Bin. Gm. Voluta vespertilio, sp. 97. 1 St.

\section{Lambis, Die Flügel-Schnecke.}

1. Alis-retractis. Mit eingezogenem Flügel. 768...1 L. Pugilis. Der Fechter. Fleisch-Schnecke. Gmel. Strombus pugilis, sp. 13. Mart. \& tab. 84 f. 838, 39. 4 Stück.

$769 \ldots-\alpha-8 S_{.}$.
$770 \ldots-\beta-3 \mathrm{St}_{\mathrm{t}}$
$771 \ldots-\gamma-1 \mathrm{St}$.




\section{Lade $37 \& 38$.}

772...2 L. Venusta. Die gebandete Flügel-Schrecke. Gmel. Strombus pugilis, sp. 13. 1 St.

773...3 L. Elegantissima. Die sehr schöne Flügel Schnecke. Gm. Strombus fasciatus, sp. 9. Mart. 3 t. 78 f. $800-$ 802. Chemn. 10 t. 155 f. 1483,84 . 7 St.

774...4 L. Luhuana. Die Löhonische Flügelschnecke. Gm. Strombus luhuanus, sp. 16. Mart. 3 t. 77 f. 789, 90. 11 Stück, wovon 1 Stück durchgeschnitten.

775... $-\alpha-$ - Mart. 3 tab. 77 fig. 791. 7 St.

$776 \ldots-\beta-2$ St.

777...5 L. Decora. Die zierliche Flügel-Schnecke. Gmel. Strombus luhuanus, sp. $16 \%$ Chemn. 10 'tab. 157 fig. 1499, 1500. 6 St.

$778 \ldots-\alpha-1 \mathrm{St}$.

779...6 L. Carinata. Die ausgehöhlte Flügel-Schnecke. Gm. Strombus marginatus, sp. 15. Chemn. 10 tab. 156 1489, 90. Mart. 3 tab. 79 f. 816 . 4 St.

$780 \ldots-\infty-1$ St.

781...7 L. Succincta. Die Kanarien-Flügel-Schnecke. Gmel. Strombus succinctus, sp. 26. Mart. 3 tab. 79 fig. 815. 7 St.

782...8 I. Gibberula. Die puckligte Flügel-Schnecke. Gm. Strombus gibberulus, sp. 17. Mart. 3 t. 27 f. 795, 96. Knorr 2 t. 14 f. 3 \& 3 t. 13 f. \&. 9 St.

$783 \ldots-\alpha-1 \mathrm{St}$.

$784 \ldots-\beta-2$ St.

$785 \ldots-\gamma-1 \mathrm{St}$.

7S6̂..9 L. Gibłosa. Die höckrigte Tlügel-Schnecke. Gm. Strombus gibberulus, sp. 17 a. Mart, 3 tab. 27 fig. 794. 11 St.

$787 . .-\alpha-\infty S_{t}$.

$788 \ldots-\beta-3$ St.

$789 \ldots 10$ L. Albida. Die weisse Flügel-Schnecke. 6 St.

790...11 L. Dentata. Jie gez̈̈hnelte Flügel-Schnecke. Gm. St. clavus, sp. 7. Chemn. 10 t.157 f. 1501, 2. 2 St.

$791 \ldots-\alpha-2$ St.

792...12 L. Fragilis. Die zerbrechliche Flügel-Schnecke. Gm. Strombus tridentatus, sp. S0. Chemn. 10 tab. 157 fig. 1503. I St。 
793...13 L. Picta. Die gemahlte Flügel-Schnecke. Gm. Str, latissimus, sp. 21. Mart. 3 t. 39 f. 874. 2 St.

793a..13 a. L. Ventricosa. * Die bauchigte Flügel-Schnecke. 1 Stück.

704...14 L. Rosea. Die rosenrothe Flügel-Schnecke. 1 St. $795 \ldots-\infty-1$ St.

796...15 L. Lentiginosa. Die Sommersprosse. Gm. Strombus lentiginosus, sp. 8. Mart. 3 t. 91 f. 892. 3 St.

797. .16 L. Rubiginosa. - Die rostfarbene Flügel-Schnecke. $2 \mathrm{St}$. 798...17 L. Coriacea. Die leberfarbene Flügel-Schnecke. 1 St. 799...18 ! L. Puellaris. Die jugendliche Flügel.Schnecke.

1 Stück.

800...19 L. Grisca. Die graue Flügel-Schnecke. 2 St. $801 . .20$ ! L. Cingulata. Die gegürtelte Flügel-Schnecke. 2 St. 802...21 L. Rana. Der Frosch. Gm. Strombus lentiginosus, sp. 8. Mart. 3 tab. 80 fig. 82\%, 28. 7 St.

$803 . . .-\alpha-2$ St.

804...22 L. Pipa. Die Kröte. Gm. Strombus lentiginosus, sp. 8. Mart. 3 t. 80 f. 825,26 . 2 St. $805 \ldots-\approx-1$ St.

805...23 L. Labiata. Die Dicklippe. Gm. Strombus urceus, sp. 29. Mart. 3 tab. 78 fig. $804,5.8$ St.

807...24 L. Urceus. Der Schwarzmund. Gm. Strombus urceus, sp. 29. Mart. 3 tab. 78 fig. 803-6. Innorr 3 tab. 13 fig. 5, 7 St.

808...25 L. Cornea. Die hornigte Flügel-Schnecke. Gmel. Strombus fasciatus, sp. 9. Mart. 3 t. 82 fig. 833, 34. 8 St.

\section{Lade 39.}

809...26 L. Carnaria. Die fleischfarbene Flügel.Schnecke, Gm. Stromb. fasciatus, sp. 9. Mart. 3 t. 91 f. 89 . 3 Stück.

2. Alis expansis. Mit ausgedehnten Flügel. 810...27 L. Turrica. Die gethürmte Flügel-Schnecke. Mart. S t. 84 f. $841,42.1^{\text {S St. }}$

$811 \ldots-\infty-1$ st.

812...28 L. Curruca. Die Mücke. Gm. Stromb. gallus, sp.11. Mart. 3 t. 83 f. 836, 37. Favanne t. 21 f. A.4. 4 St。 $813 . . .-\propto-6 \mathrm{St}$. 
814...29 L. Bulla. Der Kampfhahn. Gm. Stromb. auris Dianae, sp. 12. Mart. 3 t. 84 f. 840.7 St.

815...s0 L. Stiva. Die Pflugsterze. Gm.Stromb.auris Dianae, sp. 12. 2 St.

816...31 L. Auris Diana. Der Dianen-Flügel. Gm. Stromb. auris Dianae, sp. 12. Mart. 3 t. 84 f. $838,39.3$ St.

${ }^{*} 317 . .1 \alpha-$ - sehr selten. 1 St.

$818 \ldots-\beta-\beta$ - 5 S.

819...32 L. Buris. Das Eselsohr. Gm. Stromb, auris Dianae, sp. 12. 3 St.

820...33 L. Avatrum. Der Pflug. Gn. Stromb. auris Dianae, Mart. 148\%, 88. 1 St.

821...34 L. Vomer. Das Pfugschareisen. Gm. Stromb. auris Dianae. Mart. 1495, 86. 1 St.

822...35 L. Gallus. Der Engelsflügel. Gm. Stromb. gallus, sp. 11. Mart. 3 t. 84 f. 841 , 42 . Knorr 4 t. 12 f. 1. 5 Stück.

823...36 L. Velum. Das Segel. Gm. Strombus. gallus, sp.11. Mart. 3 t. 85 f. 846, 47. 3 St.

$824 \ldots-\infty-2$ St.

\section{Lade 40, a. b.}

825...37 L.Velamen. Die eingerollte bunte Flügel-Sclnecke. Gm. Stomb. gallus, sp. 11. 2 St.

826...38 L. Lobata. Die zerrissene Flügel-Schnecke. Gmel. Stromb. gallus, sp. 11. Chemn. 10 t. 158 f. 1506, 1507 . 2 Stück.

827...39 L. Latissima. Die grosse Flügel-Schuecke, mit breitumschlagener Lippe. Gm. Stromb. latissimus. sp. 21. Mart. 3 t. 82 f. $832,33.1$ St.

828...40 I. Canarium. Das Täubchen. Gm. Stomb. canarium, sp. 24. Mart. 3 t. 79 f. 817,18 . Knorr 1 t. 18 f. 5 . 3 Stilck.

$829 \ldots-\alpha-6$ St.

$830 \ldots 41$ L. Cenariensis. Der Canarienvogel. 1 St.

831 ...42 L. Canaria. Der weisse Canarienvogel. 1 St. $832 \ldots \ldots-\alpha-$ - Selır monströs. 1 St.

833...43 L. Turturella. Das Turteltïubchen. Gm. Stromb. canarium, sp. 24. Nart. 3 t. 79 f. 817. 2 St.

831...44 L. Epidromis. Das.Besamssegel. Gm. Stromb. epidromis, sp. 22. Mart. 3 t. 79 f. 821.7 St. 
835...45 I. Plicata. Der Wetterhahn. Gm. Stromb. vittatus, sp. 25. $\delta$. Chem. 10 t. 157 f. 1496. 2 St.

836...46 L. Minimus. Die kleine Flügel-Schnecke. Gmel. Stromb. minimus, sp. 23. Chemn. 10 t. 156 f. 1491, 1492. 2 St.

$837 . . .-\alpha-1$ St.

838...47 I. Vittata. Die Windfahne. Gm. Stromb. vittatus. sp. 25. $\%$ Mart.3 t.822, 23. Chemn. 10 t.155 f. 1481, 82. 6 St.

839...48 L. Contorta. Der Thurmflügel. Gm. Stromb. clavus, sp. 7. Mart. 4 t. 159 f. 1502, 3, 4. 3 St.

840...49 I. Accipitrina. Der Habichtsfügel. Gmel. Stromb. costatus, sp. 32. Mart. \& t. 81 f. 829 . 2 St. ?

$841 \ldots-\alpha-\ldots$ - 1 St.

$842 . .50 \mathrm{~L}$. Gigas. Die grosse Flügel-Schnecke. Gm.Stromb. gigas, sp. 20. Mart. 3 t. 80 f. 824. $2 \mathrm{St}$. $843 \ldots-\infty-2 \mathrm{St}$.

3. Alis digitatis. Mit gespaltenen Flügel.

844...51 L. Lambis. Die Krabbe. Gm. Stromb. lambis, sp.5. Mart. 3 t. 86 f. 855 \& t. 91 f. 888.12 St.

\section{Lade 41. a. b.}

845...52 L. Lamboides. Der Krebs. Gm. Stromb. lambis, sp. 5. 2 St.

846...53 L. Cerea. Der weisse hrebs. Gm. Stromb. lambis, sp. 5. 1 St.

847...54 I. Davilae. Die Lumpenkrabbe.

848... - $\propto$ - Davillae Pl. 14 f. 2 St.

849... - $\beta$ - Davillae Pl. 13 f. Chemn. 10 t. 158 fig. 1512. $1 \mathrm{St}$.

$850 \ldots-\gamma-3 \mathrm{St}$.

851...55 L. Bryonia. Die abgestumpfte Flügel.Schnecke. Gm.

Stromb. bryonia, sp. 33. Mart. 3 t. 93 f. 904, 905, 2 Stück.

$852 \ldots-\alpha-\alpha-1$ St.

853...56 L. Radix. Die Wurzel. Gm. Stromb. bryonia, sp. 33. a. Chemn. 10 t. 159 f 1514,15 , 2 St.

854...57 L. Maculata. Der bunte Krebs. Gm. Stromb. lam= bis, sp. 5. B. Mart. 3 t. 87 f. 858 , 59. 2 St. 
855...58 L. Millepeda. Der Krellerwurm. Gm. Stromb. millepeda, sp. 6. Mart. 3 t. 89 f. 861, 62. 4 St.

\section{Lade. 42.}

$856 . . .59$ L. Chiragra. Der Podagra-Krebs. Gm. Stromb. scorpius, sp. 4. Mart. 3 t. 88 f. 860.4 St.

$857 \ldots 60$ L. Scorpius. Der Scorpion. Gm. Stromb. lambis. Chemri. 10 t. 158 f. 1508,9 . \& St.

$858 \ldots 61$ L. Arbritica. Die kleine Teufelsklaue. Gm. Stromb. chiragra, sp. 3. Mart. 3 t. 87 f. 857 . 2 St.

$859 \ldots$ - $\%$ - 1 St.

860...62 L. Harpago. Der Botsmannshacken. Gm. Stromb. chiragra, sp. 3. 4 St.

$361 \ldots-\infty-1$ St.

\section{Lade 43.}

* Mit offnen Griffen.

852...62 L. Harpago. 1. -2 St.

863... - 2. - - Mart. 3 t. 92 f. 895, 96. 3 St,

$864 . .$. - 3. - - 1 St.

$866 \ldots$ - 4. - - 1 St.

$867 . . .5$ - 5. - 3 St.

869...63 L. Pes-Pelicani. Die Fünffinger-Schnecke. Gmel. Stromb. pes pelicani, sp.2. Mart. \& t. 85 f. 848-850 10 Stück.

$870 \ldots-\alpha-1 \mathrm{St}$.

$871 \ldots-\beta-2$ St.

4. Alis lobato laciniatis. Mit zerlapten Flügel. I 872...64 L. Lobata. Die Flügel-Schnecke mit gefaltener Lippe; Gm. Stromb. lambis, sp. 5 b. Mart. 3 t. 92 f. 902. $1 \mathrm{St}_{\mathrm{o}}$ $873 \ldots 65$ L. Undulata. Die Flügel-Scknecke, mit wellenförmi。 ger Lippe. Gm. Stromb. lambis, sp. 5. Matt. 3 t.92 f. 898. 2 St.

874...- L. ๙ - - verstümmelte Exemplare. 5 St. $875 . . .66$ L. Hermaphroditae. Die Zwitter-Flügel-Schnecke, Gm. Stromb. lambis, sp. 5. I Sto $876 \ldots-\%-1 \mathrm{St}_{\text {. }}$ 
877...67 L. Laciniata. Dio Flügel-Schnecke mit gerunzeiter

Lippe. Gm. Stromb. lambis, sp. 5. a. 1 St.

878... - - uncomplete Exemphare. Mart. 3 t. 91 f. 888 , 889. 7 St.

\section{Lade 44.}

\section{Dentalium. Der Zahn.}

879...1 D. Aprinzm. Der Widderzahn. (Mart. Conch. tab. fig. 4. B. incst. Linnaei sp. 2.) $2 \mathrm{St}$.

880...2 D. Eburneum. Der elfenbeinerne Zahn. I St.

881...3 D. Elephantinum. Der Elephanten-Zahn. Gm. Den. tal. elephantinum, sp. 1. Mart. 1 t. 1 f. 5 a. 3 St.

882...4 D. Lupinum. Der Wolfszahn. Gm. Dental. striatulum, sp. 13. Mart. 1 t. 1 f. 5. 1 St.

883...5 D. Entalis. Der kleine Zahn。 Gm. Dental. fasciatum, sp. 10. Mart. 1 tab. 1 fig. 3 b. 2 St.

\section{Verpa. Der Venus-Schaft.}

884...1 V. Fenis. Der Venus-Schaft. Gm. Serpula penis, sp. 17. Mart. I. tab. 1 fig. \% Finorr \& tab. 23 fig. 1 \& 6 tab. 40 fig. 1. 4 St.

\section{Serpula, Die Wurm-Röhre.}

885...1 S. Intestinalis. Der dicke Darm. Gm. Serpula anguina, sp. 15 ß. Mart. 1 t. 1 f. 11.1 St.

$886 . .2$ S. Ileacea. Der dünne Darm. 1 St.

887...3 S. Clava Herculis: Die Hercules-Tieule. Naturforsch. 13 St. tab. 1 fig. 10.1 St.

888... 4 S. Duodenalis. Der 12 Finger-Darm. d'Argenv. Pl. 29 fig. E. 5 St.

889...5. S. Polita. Der glatte Darm. Mart. 1 t. 3 f. 21. 1 St. 890..6 S. Lamellata. Die blättrige Wurm-Röhre. Gm. Serpula contortuplicata, sp. 10. Mart. 3 f. 24 A. 6 St. 891...7 S. Anguina. Die geschlängelte Wurm-Röhre. Miart. 3 fig. 13 A. 3 St.

892...8 S. Porosa. Der rothe Darm. Mart. 2 f. 13 C. 1 St. 893...9 ! S. Lumbricalis. Der Zepter Jovis, d'Argenv. tib. $29 \mathrm{fig}$ J. $1 \mathrm{St}$. 
884...10 S. Colubrina. Die sclıwarze Wurm-Pölnre. Knorr tab. 22 fig. 1. 1 St.

885...11 S. Contortuplicata. Die gewundene Wurm-Pöhre. Gm. Serpula contortuplicata, sp. 10. Mart. 1 tab. 3 fig. $24 \mathrm{~A} .7 \mathrm{St}$.

896...12 S. Glomerata. Die gewichelte Wurm-Rühre. Mart. 1 tab. 19 fig. B. 5 St.

897...13 S. Coralloides. Die korallenfürmige Wurm-Röhre.

1 Stück.

898... If S. Merdaria. Der Hünerdarm. I St.

\section{Lade 45 \& 46 b. Nautilus. Die Nautel.}

899...1 N. Ponpilius. Die grosse Nautel. Gm. Naut. pom. pilius, sp. 1. Mart. 1 tab. 18 fig. 164. 9 St.

900... - - - Mart. 1 t. 18 f. 166. 7 St: abgezogen.

$901 \ldots$ - I* - Geschnitten entomologisch. 1 St.

902... - 2* - - Geschnitten mythologisch. 1 St.

$903 . . .-3^{*}-$ - Geschnitten mit Blumen. 1 St.

Die Schnitzeleien auf diesen Nautilis sind äusserst kunstroll und trefflich.

$901 \ldots 2$ N. Spirula. 12 St.

\section{Lade $4 \%$ \\ Argonauta. Der Segler.}

905...1 A. Argo. Die schmalkieligte Papier-Nautel. Gm. Ar* gonauta argo, sp. $1 \%$ Mart. 1 t. 17 f. $15 \%$. 2 St.

906...2 A. Tuberculata. Der Reisbrey-Nautel. Gm. Argon, argo, sp, 1 \&. Mart. 1 t. 17 f.-156. 2 St.

907...3 A. Paprracea. Der Papier-Nautel. Gm. Argon. arge, sp. 1. 2 St.

909...4 A. Bibula. Der Löschpapier-Nautel. Gmel. Argor. argos sp. 1 \&. Mart. 1 t. 18 f. 160 . 3 St. \& 1 St. defect.

\section{Angaria. Das Posthorn.}

1. Columella depressa. Mit gedrückter Windung. 909...1 A. Squamata. Der schuppigte Delphin. Gm. Turbo delphimus, sp. 44. Mart. 1730, 31. Geve tab. 4 fig. $24 \mathrm{~A}, 3 \mathrm{St}$. 
910...2 A. Distorta. Der rothe Delphin. Gm. Turbo distortus, sp. 45. Mart. 5 t. 175 f. 1737-39. $2 \mathrm{St}$.

911...3 A. Delphinus. Der gezachte Delphin. Gmel. Turbo. delphinus, sp. 44. Mart. 5 t. 175 f. 1734, 35. 15 St.

912...4 A. Fosea. Der rosenfarbene Delphin. Gmel. Turbo delphinus, sp. 44. Mart. 5 t. 175 f. 1733.3 St.

913...6 A.Arion. Der Arion. Gm. Turbo delphinus, sp. $44 \%$ Mart. 5 táb. 175 fig. 17.s6. 1 St.

914...5 A. Cincta. Der gebandete Delphin. Gm. Turbo del. phinus, sp. 44. Mart. 1732. 3 St.

915...7 ! A. Pentagona. Der fünfechigte Delphin。 1 St.

2. Columella elevata. Mit erhabener Windung.

916...8 A. Lineata. Das liniirte Posthorn. Gm. Helix erice torum, sp. 65. Gualt. tab. 3 fig. O. Chemn. 9 t. 132 f. $1193-95.5 \mathrm{St}$.

917...9 A. Fasciata. Das gebandete Posthorn. Gm. Helix volvulus, sp. 91. Seba Tom. III. Tab. XL. fig. 18. Chemn. 9 tab. 123 fig. 1034-66. 1 St.

918...10 A. Polita. Das gefaltene Posthorn. Gm. Helix lapicida, sp. 2. Seba Tom. 3 Tab. XL. fig. 33, 34. Ch. 9 t. 126 f. 1107.1 St.

919...11 A. Bifasciata. Das 2mal gebandete Posthorn. Chemn. 9 tab. 125 fig. 1071, 72. 1 St.

\section{Planorbis. Die Scheibe.}

920... I P. Verus. Die kleine Scheibe. Geve tab. \& fig. 21 a. b. 22, 23. 4 St.

921...2. P. Cornu Arietis. Das Widderhorn. Gn. Helix cormu arietis, sp. 41. Lister $136 \mathrm{fg}$. 40. I St.

922...3 P. Corneus. Die Horn-Scheibe. Gm. Helix cornea, sp. 35. Chemn. 9 t. 127 f. $1113-23.9$ St.

928...4 p. Serpentinus. Die geschlïngelte Scheibe.

924...4* P. Lacieus. Die weisse Scheibe.

925...5 P. Ungulinus. Die dunkelbraune Scheibe. Gm. Helix ungulina, sp. 75. Chemn. 9 t. 125 f. 1098, 99. Gnalt. 3 fig. 222. 2 St.

926...6 P. Citrinzus. Die citronengelbe Scheibe. 1 St.

$927 . .77$. Zonariuss. Die umgürtete Scheibe. Gm. Helix zonaria, sp. 63. Gualt. 3 f.LI. Tnom 5 t. 21 f.3, 4. 6 St. 
928...8 ! P. Pellucidus. Die durchsichtige Scheibe, mit einem monströsen Eingang. 1 St.

9a...9 P. Bilineatrus. Die zweybandigte Scheibe. Gm. Helix zonaria, sp. 63. 1 St.

930...10 P. Pellis-anguinea. Die Schlangen-Haut. Gm. Helix pellis serpentis, sp. 254. Chienm. 9 tab. 125 f. 1095, 96. $3 \mathrm{St}$.

931...11 P. Lapicida. Die steinartige Scheibe, aus Rupin. Gm. Helix planorbis, sp. 20. Chemn. 9 tab. 126 fig. 1102, 3. 4 St.

932...12 P. Jamaicensis. Die Scheibe aus Jamaica. Gm. Helix jamaicensis, sp. 234. Chemn. 9 t. 129 f. 1140, 45. Seba XL. fig. 3, 4, 5. 3 St.

933...13 P. Boltenianus. Die Boltensche Scheibe. Mart. 921, 922.

934...14 P. Effusus. Die vielbandige Scheibe. Gm. Helix ampulacea, sp. $43 \%$. Chemn. 9 t. 129 f.1144, 45. 8 St. 935...15 P. Cinctus. Die umwundene Scheibe. Gmel. Helix cincta, sp. 56. 2 St. (1 St, durchgeschnitten.)

\section{Lade 48.}

\section{Pomatia. Die Garten-Schnecke.}

936...1. P. Carinata. Die gelbbraune Gárten-Schnecke. Gm. Helix cornu militare, sp. 29. I St.

-...- - - Chemn. 9 t, 129 f. 1142, 43. 1 St.

937...2 P. Nivea. Die schneeweisse Garten-Schnecke. Gm. Helix nivea, sp. 176.1 St.

939...3 P. Lactea. Die milchweisse Garten-Schnecke. 2 St. 939... P. Filata. Die fadigte Garten-Schnecke. 1 St. $940 . . .-\alpha-2$ St.

911...5 P. Exotica. Die ausländische Garten-Schneche. 5 St. 942...6 P. Atomaria. Die bestaubte Garten-Schneche. Gm. H. lactea, sp. 23\%. Chemn. 9 t. 130 f. 1161. 5 St.

943...7 P. Depressa. Die platte Garten-Schneche.

$944 . .8$ P. Frondosa Die Bündelartige Garten-Schnecke. $1 \mathrm{St}$. S45...9 P. Usta. Die gelbliche Garten-Schnecke. I St. 940...10 P. Hortensis. Die lichtgelbe Garten-Schneche. Gn. Helix hortensis, sp. 109. 1 St.

947...11 P. Arvensis. Die Feld-Schnecke. Gm. Helix Mortensis, sp. 100. 1 Sta 
948...12 P. Nemorosa. Die röthlichc Garten - Schnecke. Gm. Helix hortensis, sp. 109. 1 St.

949...13 P. Silvestris. Die braungebandete Gasten-Schnecke. Gm. Helix hortensis, sp. 109. I St.

950...14 P. Rhodia. Die rosenrothe Garten-Schnecke. Gm. Helix rhodia, sp. 239. Chemn. 9 t. 132 f. 1179, 80. 1 Stiick.

951...15 P. Baltheus. Die runzliche Garten-Schnecke. Gm. Helix algira, sp. 11. Chemn. 9 t.125 f. 1093, 94. 2St. 952...16 P. Cicatricosa. Die narbigte Garten-Schnecke. Gm. Helix cicatricosa, sp. 4. Chemn. 9 t. 109 f. 923. 2 St. 953...17 P. Succinea. Die bernsteinfarbne Garten-Schnecke. Gm. Helix citrina, sp. 49. Cliemn. 9 tab. 131 fig. 1169. 1 St.

954...18 P. Ruteola. Die braunrothe Garten-Schnecke. Gn.

Helix citrina, sp. 49. Chemn. 9 t. 131 f. 1173. 1 St. 955...19 P. Dimidiata. Die zweifarbige Garten-Schneclie. Gm. Helix citrina, sp. 49. Ch. 9 t. 131 f. 1170. 2 St. 956...20 P. Rutila. Die rothbraune Garten-Schnecke. Gmel. Helix citrina, sp. 49 . Clemn. 9 t. 131 f. 1174. 1 St. 957...21 P. Flaveola. Die blasgelbe Garten-Schnecke. Gmel. Helix citrina, sp. 49. Geve t. 26 f. 278. 2 St.

958...22 P. Citrina: Die citronengelbe Garten-Schnecke. Gm. Helix citrina, sp. 49 . v. Born t. 15 f. 3; 4., Chemn. 9 tab. 131 fig. 1170. 2 St.

959...23 P. Paleacea. Die gelbe gebandete Garten-Schnecke. Gm. Helix citrina, sp. 49. Chemn. 9 t. 131 f. 1170. 2 Stïck.

960..24 P. Straminea. Die strohfarbene Garten-Schnecke. Gm. Helix citrina, sp. 49. Geve t. 24 f. 280.4 St. 931...25! P. Imbricata. Die gereifte Garten-Schnecke. Geve tab. 24. fig. 243. 1 St.

962...26! P. Transpareus. Die durchsichtige Garten-Schnecke. 1 Stück.

\section{Janthina. Die Wald-Schnecke.}

1. Elenata. Mit erhabenem Zopf.

963... I Jiolacea. Die blaue WaId-Schnecke, oder Qualje Böotes. Gm. Helix janthina, sp. 103. Knorr 2 tab. so fig. 2, 3. $4 \mathrm{St}$. 
54

$964 . . .2$ ! J. Singalaris. 'Die besonders schöne Wald-Schnecke. sehr selten. 1 St.

955...3 J. Turbinoidea. Die knopfartige Wald-Schnecke. I St. 966... J. Limbata. Die gesäumte Wald-Schnecke. 3 St.

967...5 J. Cytherea. Die zarte Wald-Schnecke. Chemn. 9 tab. 123 fig. 1059. 1 St.

968...6 J. Pellucida. Die durchsichtige Wald-Schnecḱe. 1 St.

2. Depressiusculc. Mit gedrücktem Zopf.

969...7 J. Urru. Der Aschentopf: 1 S.

$970 \ldots 8$ J. Bombarda. Die Kugel-Schneche. 3 St.

971...9. J. Sclopetaria. Die Kleine Kugel. 3 St.

$-\ldots 9^{*}-1$ St.

972...10 J. Pomatia. Die gebạndete Wald-Schnecke. Gm.H. pomatia, sp. $4 \%$ Chemn. 9 t. 128 f. $119 \%$. 4 St.

973...- - - - varietas finistra. Chemn.9 tab. 108 fig. $908,9,10.2$ St.

974...- $\beta !$ - - varietas elongata. Gm. Helix scalatis, sp. 116. Chemn. 9 t. 128 f. 1139 Nio. 1. 1 St.

975...11 J. Flammeola. Die geflammte Wald-Schnecke. Gm. Helix ligata, sp. 5\%. Ch. 9 t. 128 f. 113\% 11 St.

*976..12 !! J. Jamaicensis. Die Jamaica-Schnecke. Gmel. H. jamaicensis, sp. 234. Chemn. 9 tab. 199 fig. 1140, 41. 1 Stück.

$977 \ldots-\infty-1$ St.

978...13 J. Pinearia. Die linirirte Wrald-Schnecke. 2 St.

979...14 J. Picta. Die gezeichnete Wald-Schnecke. Gm. H. picta, sp. 189. 1 St.

$980 \ldots-\%$ - -1 St.

981...15 J. Cortex citri. Die Citronenschale. Gm. H. picta, sp. 189. Chemn. 9 t. 130 f. $-1162,63.1$ St.

\section{Lade 49.}

\section{Lampadion. Die Lampe.}

1. Fauce simplïci. Mit gewöhnlicher Oeffnung. 982... L L. Caracolla. Die braune Lampe. Gm. H. caracolla, sp. 25. Chemn. 9 t. 125 f. 1091.6 St.

983...2 I. Testaceum. Die Nachtlampe. Gm. II. caracolla, sp. 2j. Chemn. 9 t. 125 f. 1092.3 St. 
984...3 I. Mtarginatum. Dịe gebandete Lampe. Gm. Helix marginella, sp. 162. v. Born t. $14 \mathrm{f} .7,8$. 2 St.

985...4 L. Orbiculare. Die runde Lampe. Gm. H. marginella, sp. 162. Chemn. 9 tab. 125 fig. 1097. 1 St.

986...4 * L. 3 Lineatum. Die gedrehte Lampe. 2 St.

987...5. L. Sinicum. Die gekehlte Lampe. 1 St.

988...6 I. Chinense. Die chinesische Lampe. Gm. Trochus vestiarius, sp. 75 in allem 15 Stück. Chemn. 5 tab. 165 fig. 1601 a. b. c. f. g. h. Lister tab. 149 \& 658 fig. 44-50. Wie unten bemerkt.

$989 \ldots-1 . \ldots$ - 1 St.

$990 \ldots-2 .-1$ St.

$991 \ldots-3 .-3 \mathrm{st}$.

$392 . . .4 .4 \mathrm{St}$.

$993 . . .5$ - 5. - $1 S_{t}$

$994 \ldots-6 . .-3 \mathrm{st}$.

$995 \ldots-7 .-4$ St.

2. Fauce ringente. Mit weiter Oeffnung. *996...9 L. Lucerna. Die gedruckte Kugel - Lampe, sehr sel\% ten. 2 St.

*997...9 a. L. Tumidum. Die Kugel-Lampe, selr selten. I St. *998... 10 ! L. Labyrinthus. Das Labyrinth, sehr selten. Favanne tab. LXIII. fig. 2. $2 \mathrm{St}$.

*999...11 L. Turritum. Die Schnecken-Lampe. Gm. Helix sinuata, sp. 23. sehr selten. Chemn. 9 tab. 126 fig. 1110-12. 1 St.

$1000 \ldots-\infty-1 \mathrm{St}$.

*1001..12 L. Ringens. Die Begräbniss-Lampe. Gm. Helix ringens, sp. 22, sehr selten. Chemn. 9 tab. 109 fig, 919, 20. 2 St.

$-\ldots 12 a_{0}-2$ St. diverse:

\section{Architectonica. Die Perspectiv-Schnecke.}

1002... I A. Perspectiva. Die Perspectiv-Schnecke. Gm. Tro. chus perspectivus, sp. 3. Chemn. 5 t. 192 f. 1691, 9 9. Knorr 1 tab. 11 fig. 1, 2. 5 St.

$1003 \ldots-\infty-2 \mathrm{St}$.

$1004 \ldots-\beta \Rightarrow-$ Chemn, 5 t, 172 f. 1695, 94. 10 St. 
*1005..2 ! A. Nobilis. Die geperlte Perspectiv-Schnecke. Gm. T. perspectivus, sp. $\triangle$, sehr selten. Chemn. 5 tab. 172 fig. $1695,95.1$ St.

1006...3 A. Gothica. Die branne Perspectiv-Schnecke. Gm. T. stramineus, sp. 59. Chemn. 5 tab. 172 fig. 1699. Lister Conch. tab. 635 fig. 23. 3 St.

$1007 . . .4$ A. Radiata. Die weissfiussigte Perspectiv-Schnecke. Gm. T. hybridus; sp. 4. Chemn. 5 tab. 163 . fig. 1704, 5. Gere t. 25 f. 274 a. 74 b. :3 St.

\section{Lade 50 \& a.}

\section{Astraea. Das Sonnenhorn.}

*1008..1 ! A. Imperialis. Das kaiserliche Sonnenhorn. Gm. Trochus imperialis, sp. 63 , sehr selten. Clemn. 5 tab. 173,74 fig. $1714,15.1 \mathrm{St}$.

*1009..2 A, Sirius. Der Siriusstern. Gm. T. solaris, sp. 15, var. sehr selten. $1 \mathrm{St}$.

1010...3 A. Polaris. Das Sonnenhorn des Rumpfs. Gm. T. solaris, sp. 15. Chemn. 5 t. 173. f. $1700,1701.3 \mathrm{St}$.

1011...4 A. Calcar, Der Sporn. Gm. Turbo aculeatus, sp. 48. Favanne Pl. 12 fig. E. 4. Chemri. 5 tab. 164 fig. $1554,55.4$ St.

$1012 \ldots-\alpha-3 S_{t}$.

1013...5 A. Nicobarica. Der nicobarische Sporn. Gm. T. aculeatus, sp. 48 . Chemn. 5 t. 164 f. 1554,55 . \& St.

$1014 \ldots 5$ * A. Micans. Das grüne Sonnenhorn. 1 St.

1015...6 A. Venus. Die untergehende Sonne. 3 St.

1016...7 A. Phoebia. Die aufgehende Sonne. Gm. Trochus solaris, sp. 15 \&. Cl. 5 t. 174 f. $1716,17.8$ St.

1017...8 A. Corrusca. Das glänzende Somenhorn. Gm. Trochus intermis, sp. 62. Chemm. 5 t. 173 f. 1712, 19. 1 Stück.

$1018 \ldots$ - $\alpha-1$ - 1 St.

1019...9 A. Titania. Das Titanshorn. Gm. Tr. solaris. sp. 65. Chemn. 5 t. $1 \% 4$ f. 1718,19 . \& St.

1020...10 A. Lapidifera. Der Steinträger. Gm. Trochus con. chiliophorus, sp. 110. Chemn. 5 tab. 172 fig. 168s, 1689. $1 \mathrm{St}$. 
1021...11 A. Conchyliophoia. Der Schneckenträger. - Gmel. Trochus conchyliophorus, sp. 110. Chemn. 5 t. 172 f. 1688-90. Tavanne Pl. 12 fig. C. 1. 1 St.

1022...12 A. Corallophora. Der Corallenträger. Gm. Trochus conchyliophorus, sp. 110. 1 St.

\section{Trochus, Der Kräusel.}

1. Unbilico perforata. Mit offenem Nabel. 1023...1 T. Niloticus. Der Nilkräusel. Gm. Trochus niloticus, sp. 1. Chemn. 5 t. 167 f. 1605.1 St.

1024...2 T. Flammeus. Der geflammte Kräusel. Gm. Trochus niloticus sp. 1. Chemn. 5 t. 167 f. 1606, \%. 9 St. 1025...S T. Undatus. Der gewellte Kräusel. Gm. T. niloticus, sp. 1. Geve t. 6 f. 44 a. b. 6 St.

1026...4 T. Asper. Der granulirte Kräusel. Gm. T. virgatus, sp. 83. Chemn. 5 t. 160 f. $1514,15.8$ St. 102\%...5 T. Grandinatus, Der scharfe Kräusel. Gm. T. maculatus, sp. 2. Chemn. 5 t. 168 f. 1616. 1 St. $1028 \ldots-\infty-2$ - -4 .

1029..66 T. Stellaris: Der gestirnte Kräusel. Gmel. Trochus stellatus, sp. 25. Chemn. 5 t. 169 f. 1630. 2 St.

1030...7 T. Granularis. Der.bunte rauhe Kräusel. Gm. Tr. radiatus, sp. $33 \%$ Chemn. 5 t. 170 f. 1642 a. b. 11 St 1031...8 T. Fernalis. Der grasgrüne Kräusel. Gm. T. vernus sp. 22. Chemn. 5 t. 169 f. $1625,26.1$ St.

1032...9 T. Nasutus. Der spitze Krräusel. Gm. T. conspersus, sp. 23. Chemn. 5 t. 169 f. $162 \%$ 。

\section{Lade 50 a.}

1033...10 T. Guttatus. Der tropf. Kräusel. Gm. T. consper. sus, sp. 23. Geve t. 7 f. 50 a. b. 3 St.

*1034..11 T. Castrensis. Der geperlte Kräusel. Gm. T. ochroleucus,. sp. 25, sehr selten. Chemn. 5 tab. 169 fig; 1629. 1 St.

1035...12 T. Tentorialis. Der gefaltene Kräusel. Gm. Trochus ochroleucus, sp. 25. I St.

*1036..13 T. Turboidea. Der geschnürte Kräusel. Gm. Tr. magus, sp. 7 , sehr selten. Chemn. 5 tab. 171 fig. 1658. \& Sto 
*105\%.14 T. Tectum chinense. Das chinesische Dach, sehr selten. 1 St.

1033...15 T. Striatus. Der gestreifte Kräusel, sp. 78. 1 St.

1039...16 T. Turritus. Der gethürmte Kräısel. Gm. T. py• Jamis, sp. 39. Chemn. 5 t. 170 f. 1652.1 St.

1040...17 T. Verrucosus. Der warzigte Kräusel, 2 St. $1041 \ldots 18$ T. Interruptus. Der kleinste Kräusel. "1 St.

*1042..19 T. Trochlea. Der gedrechselte Krräusel, sehr selten。 1043...20 T. Declivis. Der schiefe Kräusel. Gn. T. aegypticus, sp. 41. Chemn. 5 t. 171 f. 1663, 64. 1 St.

1044...21 T. Lunatus. Der ausgekehlte Kî́räusel. Gmel. Tr. jujubinus, sp. 19. Chemn. 5 t. 167 f. 1612, 13. 2 St.

\section{Unbilico clauso. Mit geschlossenem Nabel.}

1045...22 T. Plicatus. Der gefaltete Kräusel. Gm. Tr. inzbricatus, sp. 93. Chemn. 5 t. 162 f. 1531.2 St.

1046...23 T. Rugosus. Der runzligte Kräusel. Gm. T. imbricatus, sp. 93. Knnorr V. 3 t. 29 f. 1, 2. \& St.

$1047 \ldots . .23$ * T. Spinosus. Der dornigte Kräusel, sp. 18. 1 St. 1048...24 T. Anilis. Der alte Trräusel. Gm. T. americanus, sp. 94. Chemn. 5 t. 162 f. $1594,35.4$ St.

*1049..25 T. Forskohlii. Der Forskohlsche Kräusel. Gm. T. foveoletus, sp. 84 3 . selir selten. Chemn. 5 tab. 161 f. $1518,19.2$ St.

1050...26 T. Gothicus. Der Gothische Kriäusel. Gm. Trochus manritianus, sp. 99. Chemn. 5 ti 163 fig. 1547,48 . 9 Stück.

$1051 \ldots 27$ - 1 St.

1052...28 T. Fenestratus. Dər Plauten-Kräısel. Gm. Tr. fenestratus, sp. 100 Chemn. 5 t. 163 f. 1589, 90. 5 St.

1053...29 T. Papillaris. Der scliäckigte Kräusel. Gmel. Tr. virgatus, sp. 83. Chemn. 5 t. 160 f. $1514,15.2$ St. 1054...30 T. Obeliscus. Der Obelisk. Gm. T. obeliscus, sp. 81. Chemn. 5 to 160 f. $1510-12.2$ St.

1055...31 T. Pyramis. Der spitzzulaufende hiräusel. Gm. T. niloticus, 'sp. 1. Ch. 5 t. 167 f. 1608 . 2 St.

1056...32 T. Variegatus. Der gerändete Krräusel, sp. 60.

1057...33 T. Aeruginosus. Der kupfrige Kräusel. Gm. Tr. zizyphinus, sp. 80. v. Born Mus. Caes. Vind, tab. 12 fig. 9, 10 (granulatus.) 1 St. 
*1058..33 * ! T. Helicinus. Der stłmpfspitzige Kräusel, sp. 101. $1 \mathrm{St}$.

*1059..32 ** ! T. Globulus. Der stumpfe Kräusel. Gm. He• lix Cookiana, sp. 230, sehr selten. Lister tab. 62 fig. 60. Chemn. 9: t. 122 f. 1049, 50. 1 St.

1060...34 T. Tornatus. Der abgedrehte Kräusel, Gm. Trochus, jujubinus, sp. 19 var. Chemn. 5 t. 166 f. 1595 3 Stück.

1061...35 Jujubinus. Der Jujuben -Kräusel. Gm. Tr. jujubi nus, sp. 19. Chemn. 5 t. 166 f. 1594.4 St.

1062...36 T. Conulus. Der hellbraune Kräusel. Gm. T. conu• lus, sp. 79. Chemn. 5 t. 166 f. 1588,6 St.

1063...37 T. Tranquebaricus. Der Tr̉anquebarische Kräusel. Gm. Trochus zizyphinus, sp. $80 \beta$. Chemn. 5 tab 166 fig. 1595, 96. 1 St.

1054...38 T. Communis. Der gemeine Träusel. Gm. Trochus conulus, sp. 79. 6 St. Abgezogen 3 St.

*1055..89 T. Novae Zeelandiae. Der neuseeländische Kräusel. Gm. T. Labio, sp. 76. Mart. 5 to 166 fig. 1579, 80. Selten.

\section{Cidaris, Der Turban.}

1. Anfractibus Spinosis. Mit dornigten Windungen. 1066...1 C. Cookii. Der Cooksche Turban. Gm. Trachus, sp. s7. Chemn. 5 t. 164 f. $1550,51.2 \mathrm{St}$.

106\% ...2 C. Pagodus. Die Pagode. Gm. Turbo pagodus: spon 12. Chemn, 5 t. 163 f. $1541,42.4$ St. $-\ldots-a-3 \mathrm{st}$.

I06s... . C. Tectum persicum. Das persische Dach. Gm. Turbo tectum persicum, sp. 11. Chemn. 5 tab. 163 fig. 1543, 44. 3 St.

1069...4 C. Spinula. Der stacliligte Turban. \& St.

1070...5 C. Arabica. Der arabiscle Turban. Gm. Trochus aegyptiacus, sp. 41. Chemn. 5 tab. 171 fig. 1663, 64. 6 Stück.

1071...6 C. Otaisensis. Der otaheitische Turban, 1 St. 1072...7 C. Rugosa. Der runzligte Turban. Gm. Turbo-rugosus, sp. 14. Chemn. 5 tab. 181 fig. 1783, 1784. 4 Stück, 
1073...8 C. Caelata. Der gemeisselie Turban. Gm. Trochus caelatus, sp. 95. Chemn. 5 t. 162 f. 1536, 37. Knorr Vergn. 5 t. 12 f. 3.8 St.

1074...9 C. Plicata. Der gefaltene Turban. Gm. Turbo ru, gosus, sp. 14. var. 6 St.

1075...9* C. Vahlii. Der stachligte Turban. Gmel. Turbo Jugosus, sp. 16 var. Chemn. 10 tab. 165 fig. 1585, - 86. 2 St.

\section{Lade $50 \mathrm{~b}$.}

2. Anfractibus rugosis. Mit gerunżelter Windung. 1076...10 C. Magus. Der Zauberer. Gm. Trochus magus, sp. 7. Chemn. 5 t. 171 f. 1656, 5\%. 3 St. 1077... - $\alpha-$ Chemn. 5 t. 171 f. 165s. 2 St. 1078...- $\beta$ - - Chemn. 5 t. 171 f. 1659. 4 St. 1079... $-\gamma-2$ St.

\section{Lade $50 \mathrm{c}$.}

1080...11 C. Tuberculata. Der grüne Turban. Gm. Trochus Tuber. sp. 77., Knorr Vergn. 1 t. 3 f. $2 \& 4$ t. 4 f. 3, 4. Chemn. 5 t. 165 f. $1572,73.4$ St.

1081... - $\alpha$ - olivenfarbigt. 2 St.

$1082 . . .-\beta-$ - grüne, sp. minos. $2 \mathrm{St}$.

$1083 . . .-\gamma-\gamma$ - gelbliche. 2 St.

*1084..12 C. Novae Zeelandiae. Der neuseeländische Turban. Gm. Trochus Cookii, sp. 97. Der eine ist gnnz abgezogen und der andere beinahe. Sehr selten. Chemn. 5 t. 164 f. $1550.2 \mathrm{St}$.

1085...13 C. Flamnea. Der geflammte-Turban. Gm. Trochus magus, sp. 7. Chemn. 5 t. 771 f. 1659. 2 St.

1086...14 C. Punctulata. Der gestippelte Turban. Gm. Tr. tesselatus, sp. 52. Chemn. 5 t. 171 f. 1683. 3 St. 1087...14* C. Lineata. Der gefleckte Turban. eod. 1 St. 1088...15 C. Granulata. Prinz Rudolph, t. 12 f. 1. Gm. Tr. asper, sp. 105. Chemn. 5 t. 166 f. 1582. 6 St. 1089...16 C. Sinica. Der chinesische Turban. $10 \mathrm{St}$. 1050...17 C. Purpurascers. Geve tab. 19 fig. 190, 191 a. b. 6 Stïck. 


\section{Turbo. Der Knopf.}

1. Fauce simplici.

A. Elevato granulati.

1091... T. Pica. Das Riessenohr. Gm. Turbo pica, sp. 39. Chemn. 4 tab. 151 fig. 1420, 21 \& 5 tab. 176 fig. 1750. 11 St. wovon 1 St. abgezogen. 1092...-a - - Chemn. 5 t. 176 f. 1751. 3 St. 1093...2 T. Sarmaticus. Das orange Auge. Gm. Turbo sarmaticus, sp. 16. Chemn. 5 t. 179 f. 1777, 78. 4 St. *1094..3 T. Taiticus. Der neuseeländische schwarze Knopf。 Gm. Turbo sarmaticus, sp. 16, sehr selten. 2 St.

\section{Lade 51,}

*1095..4 ! T. Smaragdinus. Der smaragdfarbne Knopf. Gm. Turbo smaragdus, sp. 30. Chemn. 5 tab. 182 f. 1815, 16. Selten. 4 St.

*1096..5! T. Merula. Der schwarze Knopf. Gm. Trochus sinensis, sp. 103. Chem11. 5 t. 165 f. 1564, 65. 3 St. *1097..6 ! T. Litharetrax. Der kohlschwarze Knopf. Gmel. Trochus argyrostomus, sp. 102. Chemin. 5 tab. 165 fig. $1562,63$. s St.

*1098..7 ! T. Aethiops. Der Mohr, Wie No. 6 nur genabelt und hleiner. 2 St.

1099...8 T. Agrestis. Der kleine schwarze Knopf. Gm. Tro. chus rusticus, sp. 35 . Chemn. 5 tab. 170 fig. 1645 , 46. 4 St.

1100...9 T. Tessellatus. Der gesprenkelte Knopf, Gm. Trochus tessellatus., sp. 106. Chemn. 5 tab. 166. fig. 1583-87. $10 \mathrm{St}$.

1101...10 T. Venosus. Der ausgedrechselte Knopf. $1 \mathrm{St}$. 1102...11 T, Purictulatus. Der gestippelte Knopf. 1 St. 1103...12 T. Neapolitanus. Der neapolitanische Knopf. 11St.

B. Elevati simplices. $1104 \ldots 12$ * a. T. Horticula. Der gebandete Knopf. I St. $1105 \ldots 12^{* *}$ b. T. Pileus. Der Huth-Knopf. Gm. Helix pi.leus, sp. 89. Chemn. 9 t. 122 f. 1048. 1 St. $1106 \ldots 12^{* * *}$ c. T. Sylvestris. Der umwundene Knopf. Gm. I. pileus, sp. 39 . Chemn. 9 t. 122 f. 1047. $1 \mathrm{St}$. 
1107...12 d. T. Galeris. Der gebandete Huth-Knopf. 2 St. $1108 \ldots 12$ e. T. Lineola. Der weisse Huth-Knopf. 1 St. 1109...12 f. T. Globulus. Der Kugel-Knopf. Gm. Helix coo. kiana, sp. 230. Chemn. 9 t. 122 f. $1049,50.1$ St. $1110 \ldots 12$ g. T. Iris. Der unvergleichliche Knopf. Gm. Trochus iris, sp. 86. Chemn. 5 tab. 161 fig. 1522, 23. I Stück,

\section{Elevati granulati.}

1111...13 T. Aurantiacus. Der bunte Knopf. $2 \mathrm{St}$. 1112...14 T. Granulatus. Der granulirte Knopf. 8 St. 1113...15 T. Pustulatus. Der schagrinirte Knopf. 1 St. $1114 . .16$ T. Irregularis. Der ïberzuckerte Knopf. 1 St.

*1115..17! T. Granatum. Der Granat-Apfel. T. Mart. univ. Conch. 2 f. 75." 1 St.

1116...18 T. Balaustinas. Der kleine Granat-Apfel. 1 St. *1117..19! T. Pulcherrimus. Der vortrefliche Knopf. 1 St.

*1118..20! T. Perlatus. Der geperle Knopf. Gm. Trochus diaphanus, sp. 85. Chemn, 5 tab. 161 fig. 1520, 21. 3 Stück.

1119..21 T. Magaritáceus. Der scharfe Knopf. 1 St.

1120...22 T. Untbilicalis. Der genabelte Knopf. Lister 632 fig. 21. Chemn. 5 t. $17 !$ f. 1666 . 3 St.

1121...22 * T. Littoreus. Der Hosen-Knopf. Gn. Turbo litoreus, sp. 3. Chemis. 5 t. 185 f. 1855.14 St.

1122...22 **. T. Davidis, Der lileine Knopf. Gmel. Turbo littoreus, sp. 3 \% Clemn. 5 tab. 185 fig. 1855 a. b. 1 Stück.

1123...22*** T. Groenlandicus. Gm. Turbo littoreụs, sp. 3 \%. Chemn. 5 t. 185 f. 1855 d. e. f. g. 2 St.

$1124 \ldots-1$ St.

\section{Fauce personata.}

1125...28 T. Labco. Der schäcliggte Knopf. Gnel. Trachus labeo, sp. 76. Chemn. 5 tab. 166 fig. 1579-81 $\beta$. Knory 1 t. 10 fig. \%. Geve t. 18 f. 165 a. b. 2 St.

$1125 \ldots-^{*}-2 \mathrm{St}$.

$1127 \ldots$ - *t -1 St.

$1128 \ldots$...*k - -2 St.

$1129 \ldots$ - $^{* * * *}-1$ St.

3130.. 24 T. Labellum. Der grüne Knopf. 1 Sta 
1191...24* T. Sulcatus. Der gefurchte hopf. 4 St. $1132 \ldots-\alpha-1$ St.

1133...25 T. Tornatus. Der gedrechselte Knopf. Gmel. Tr. ur'oanus, sp. 48. Chemn. 5 t. 171 f. 1679. 2 St.

1134...26 T. Pharaonicus. Die Pharao-Schnecke. Gm. Trochus Plaraonis, sp. 6. Chemn. 5 t. 171 f. 1672, 73. Fnory 1 t. 30 f. 6 \& 4 t. 26 f. $3,4.3$ St.

1135...27 T. Potiphareus. Der Kamisohl-Knopf. Gm. Trochus pharaonis, sp. 6. 2 St.

1136...28 T. Aegyptiacus. Der rothe Knopf. Gm. Trochus pharaonis, sp. 6. Geve t. 13 f. 101 a. b. 1 St.

1137...29 T. Subula. Der Hemdishnopf. Gm. Trochus nodulus, sp. 50. Chemn. 5 t. 171 f. 1681 . Geve tab. 13 fig. 104 a. b. 1 St.

1138...30 T. Senegalus. Der Knopf von Senegal. Gm. Trochus guinensis, sp. 49. Chemn. 5 t. 171 f. 1680. 1 St. 1139...31 T. Crenulatus. Der gereifte Knopf. Gm. Trochus carneus, sp. 51 . Chemn. 5 t. 171 f. 1682.4 St. 1140...32 T. Elegans. Der zierliche Knopf. 2 St.

\section{Cerion. Der Bienenkorb.}

\section{Integerrima. Mit ganzer Spitze.}

1141...1 C. Vulgare. Der gemeine Bienenkorb. Gn. Turbo uva, sp. 68. Lister 588 fig. 48. Fnorr 6 7ab. 25 fig. 4. 1 St.

1142...2 C. Uva. Das Wickelkind. Gm. Turbo uva, sp. 68. Lister 588 fig. $47 . \quad$ Mart. 4 t. 153 f. 1439. 8 St. $1143 . . .-\infty$ - - Lister 588 fig. 49. 2 St.

$1144 \ldots 2$ * C. Apiarium. Der Bienenkorb. Gm. Turbo war, sp. 68.3 St.

\section{Lade 51.}

1145...3 C. Pupa. Das spitze Wickelkind. Gm. Helix pupa, sp. 124. Gualti tab. 4 Lit. R. 1 St.

1146...4 C. Botrys. Die Maulbeere. Gm. Helix -? Lister t. 585 f. 43. Davilla 8 fig. X. X. 1 St.

1147...5 C. Bidens. Die linksgedrehte Baum-Schnecke. Gm, Turbo bidens, sp. 87. Chemn. 9 t. 112 f. $\$ 60$ fig. 1 , 2. $58 \mathrm{St}$. 
64

2. Truncata. Mit abgestumpfter Spitze:

$1148 . .6$ C. Truncatum. Das abgestumpfte orangegelbe Wickelkind. Gm. Helix crocea, sp. 243. Chemn. 9 tab. 135 fig. 1233. 2 St.

1149...7 C. Decapitatum. Das abgestumpfte, braun gebandete Wickelkind. Gm. Helix decollata, sp. 115. Chemn. 9 t. 135 f. 1255,1257 \%. 2 St.

1150...8 C. Decollatum. Das abgestumpfte weisse Wickelkind. Gm. Helix decollata, sp. 115. Chemn. 9. t.136 f. $1254,55.7 \mathrm{St}$.

\section{Epitonium. Der Zapfen.}

2. Apertura orbiculari. Mit zirkelrunder Oefnung.

*1151.. 1 !! E. Scolare. Die ächte Windeltreppe. Gm. Tufbo scalaris. sp. 62. Mart. 4 t. 152 f. 1426, 27. 1 St. Selten und schön.

1152...2 E. Breve. Die kurze Windeltreppe. Gm. Turbo sca. laris, sp. 62. 1 St.

1153...3 E. Lineatum. Die lineirte Windeltreppe. Gm. Turbo scalaris, sp. 62. I St:

1154...4 E. Medium. Die gestreckte Windeltreppe. Gn. Turbo scalaris, sp. 62.1 St.

1155...5 E. Principale. Die fein gerippte Windeltreppe. Gm. Turbo scalaris, sp. 62 ß. Mart. 4 tab. 152 fig. 1428, 29. 8 St.

1156..6 E. Vittatum. Die breitrippige Windeltreppe. Gm. Turbo clathrus, sp. $63.5 \mathrm{St}$.

1157...7 E. Clathrus, Die gemeine Windeltreppe. Gm. Turbo clathrus, sp. 63. Mart. 4 t. 153 f. 1454. 16 St.

1158...8 E. Elongatum. Die braune fein gerippte Windeltreppe. Gm. Turbo clathrus, sp. 63. 3 St.

1159...9 E. Valentini. Die Valentirs-Windeltreppe. Gmel. Turbo clathrus. sp. 63. Valentin t. 1 f. 7. 2 St.

1160...10 E. Minutum. Die dünnleibigte Windeltreppe. Gm。 Turbo clatlurus, sp. 68. Mart. 4 t. 155 f. $1435,36$. 2 Stück $_{\text {. }}$ 
Lade. 52.

2. Apertura rolundata. Mit ründlicher Oeffnung.

1161...11 E. Duplicatum. Der doppelt gereifte Zapfen. Gm. Turbo duplicatus, sp. 79. Mart. 4 tab. 151 fig. 1414. 11 St. wovon 1 St. durchgeschnitten.

1162...12 E. Acutangulum. Der scharfwinkligte Zapfen. Gm. Turbo acutangulus, sp. 73. Mart. 4 t. 151 f. 141 . Y̌norr 3 tab. 19 fig. 5. 1 St.

1163...13 E. Dubium. Der braune Zapfen. Gin. Turbo replicatris, sp. 77. Mart. 4 t. 151 f. 1412 . Knorr 6 t. 25 f. 3. 5 St.

1164...14 E. Terebra. Der Bohrzapfen. Gm. Turbo terebra, sp. 81. Mart. 4 t. 151 f. 1415 . Chemn. 10 tab. 165 fig. 1591. 5 St.

1165...15 E. Timpanotonor. Der Trommel-Zapfen. Gmei. Turbo terebra, sp. 81. Mart. 4 t. 151 f. 1416. 8 St. 1166...16 E. Tornatum. Der gedrechselte Zapfen. Gm. Tnr. bo terebra, sp. 81. d'Argenv. t. 14 f. E. 3 St.

1167...17 E. MTonoceros. Der dünnschaligte Zapfenı. Gmel. Turbo terebra, sp. 81 . Mart. 4 t. 151 f. 1419. 12 St. 1 St. durchgesch nitten.

1168...18 E. Infunitum. Der Zapfen ohne Ende. Gm. Turbo terebra, sp. 81. 2 St.

1169...19 E. Imbricatum. Der Senk-Zapfen, Gm. Turbo imbricatus, sp. 76. Mart. \& t. 152 f. 1422. Knorr? t. 27 f. 1 \& 6 t. 25 f. 2.3 St.

1170...20! E. Squamatum. Der Taschen-Zapfen. Lister 589 No. 53. 1 St.

1171...21 E. Rugosum. Der runzligte Zapfen. Gm. Turbo duplicatus, sp. 79. Mart. 4 t. 151 f. 1414.1 St.

1172...22 E. Var̉iegatum. Der braun und weiss geflechte Zapfen. Gm. Turbo variegatus, sp. 82. Mart. 4 t. $15 \%$ f. 1423 . Innow 6 t. 16 f. 8. 3. St.

1173...25 E. Margaritaceum. Der geperlte Zapfen, Gmel. Turbo variegatus, sp. 82. var. $2 \mathrm{St}$.

117s...24 E. Brunueum. Der dunkelbraune-Zapfen. I St, 1175...25 E. Acutum. Der weisse scharfgerillte Zapfen. Gm. Turbo duplicatus; sp. 79. $1 \mathrm{St}$. 
1176..26 E. Marmoreum. Dex genarmelte Zapfen. Gmel. Turbo imbricatus, sp. 76. Mart. 4 tab. 152 fig. 1422, 23. 7 St.

$1177 \ldots-\alpha-1 \mathrm{St}$.

1178...27 E. Eburneum. Der elfenbeinerne Zapfen. 2 St.

$1179 . .28$ E. Vitreum. Der gläserne Zapfen. 2 St. 1180...28 * E. Pusillum. Der kleine Zapfen. 1 St.

2. Apertura ovato-elongato effusa. Mit eyrunder Oeffnung.

1181...29 E. Maculatum. Das dicke Tigerbein. Gm. Buccinum maculatum, sp. 130. Mart. 4 tab. 153 fig. 1440. 7 Stück.

1182...30 E. Tigrinum. Das lünne Tigerbein. Gm. Buccin. tigrinum, sp. 140. Mart. 4 t. 154 fig. 1418. 3 St.

1183...31 E. Rubiginosum. Die rostige Penne. 2 St.

1184...32 E. Striatum. Die gestreifte Penne. Gmel. Turbo striatulus, sp. 67. 1 St.

1185...33 E. Vittatum. Die Kopfnadel. Gm. Buccinum vittatum, sp. 134. 3 St.

*1186..31 ! E. Novae Zeelandiae. Die neuseeländische Pène. 6 Stück.

1187...35.E. Lacteum. Die milchweisse Penne, Gn. Turbo lacteus, sp. 66. 1 St.

1188...s6 E. Chalybeotum. Die Stahl-Mante!. Gm. Buccinum chalybeum, sp. 159. Rumpf. t. 29 fig. Q. 3 St.

$1189 . . .-\alpha-3$ St.

1190.. 37 E. Lineatum. Die liniirte Penne. 1 St.

1191...38 E; Guttatum. Die gefleckte Penne. Gm. Buccinum maculatum, sp. $130 \%$. Mart. 4 tab. 153 fig. 1442. 2 Stïck.

1192...59 E. Dimidiatum. Die orange Penne. Gm. Buccin. dimidiatum, sp. 138. Mart. 4 t. 154 f. 1444. 4 St.

1193...40 E. Feldmanni. Die Teldmannsche Penne. Gmel. Bucc. subulatum, sp.131 \%. Mart. 4 t.154. 1446. 2St, 1194...41 E. Subulatum. Die braungeflechte Penne. Gmel. Buccinum subulatum, sp. 131. \& St.

1195... - \& - - Mart. 4 tab. 153 fig. 1441. 10 St.

1196...42 E. Creñulatum. Die gekrönte Penne. Gm. Buccinum crenulatum, sp. 152. Mart. 4 tab. 154 fig. 1445. 10 Strïck. 
1197...43 E. Acusissimum- Die sehr spizze Penne. Gm. Buc. cinum commaculatum, sp. 143. 9 St.

1198... - - - Mart. 4 tab. 154 fig. 1452. 3 St.

1199...44 E. Fissum. Die Packnadel. Gmel. Buccinum succinctim, sp. 142. Mart. 4 t. 154 f. 1451. 7 St.

1200...45 E. Anile. Die gelbbraune Penne. Gmel. Bucctnum strigilatum, sp. 135. Mart. 4 t. 155 f. 1456.1 St.

1201...46 E. Cancellatum. Die gegitterte Penne. Gm. Buccinum strigilatum, sp. 135. Mart. 4 tab. 155 fig. 1456. 1 Stück.

1202...47 E. Pulchellum. Die schöne scheckigte Penne. 3 St. 1203...48 E. Miuztum. Die kleine Penne. Gmel. Buccinum hastatum; sp. 144. Mart. 4 t. 154 f. 1454. 3 St.

\section{Plotia. Die halbe Penne.}

1204...1 P. Turrita. Die knobligte halbe Penne. I St. 1205...2 P. Lurida. Die schwarzgraue halbe Penne. 2 St. 1206...3 P. Minuta. Die kleinliche halbe Penne. Gm. Strombus vibex, sp. 42. Chemn. 9 t. 136 f. 1261, 62. 2 St. 120\% .... P. Fuscata. Die dunkelbraune halbe Penne. Clremn. 9 tab. 135 fig. I229. v. Born t. 16 f. 1\%. 2 St. 1208...5 P. Atra. Die schwarzbaune halbe Penze. Gmel. Strombus ater, sp. 39. Chemn. 9 t. 135 f. 182\%, 1 St. 1209...6 P. Fossilis. Die verkistkte halbe Penme. $1 \mathrm{~S}$. 1210...7 P. Dentata. Die gezühnelte lsalbe Penne. 1 St. 1211...8 P. Virginea. Die gestrechte Prinzen-Flagge. Gmel. Bulla virginea, sp. 24. IChemn.' 9 tab. 117 fig. 1000 , 1003. 5 St.

1212...9 P. Puellaris. Die Prinzen=Flagge, Gm. Bulla viyginea, sp. $24 \delta$. Chemn. 10 t. 173 f. $1682,83.4$ St. 1219...10 P. Lineata. Die liniirte lalbe Penne. Gmel. Trochus dolabratus, sp. 119. Chemn, 5 tab. 167 fig. 1503, 4. 10 St.

1214...11 P. Filata. Die umwindene lialbe Penne. Gmel. Trochus dolobratus, sp. 113. 2 St.

1215...12 P. Punctata. Die sestippelte halbe Fenne Gmel. Trochus dolobratrs, so.113. I. St. 
1216...13 P. Vittata. Die Kopf-Penne. Gm. Buccin. vittatum, sp. 134. Mart. 4 tab. 155 fig. 1452. 12 St.

1217... - $\alpha-4$ St.

1218... 14 P.. Tranquebarica. Die Penne von Tranquebar. Gm. Buccin. vittatum, sp. 134. Mart. 4 tab. 155 fig. 1463. 4 Stück.

1219...15 P. Dubia. Die Bastard-Penne, 3 St.

\section{Strombus. Die Nadel.}

1. Rostro recto. Mit grader Schnautze.

1220...1 S. Palustris. Der Löffel. Gmel. Strombus palustris, sp. 3.. Mart. 4 tab. 156 fig. 1472. 7 St.

${ }^{*} 1221,$. - - - selir selter. 2 St.

1222...1* S. Telescopium. Das Telescop. Gm. Trochus telescopium, sp. 112. Chemn. 5 tab. 160 fig. 1507, 8. 8 Stück.

1223...- - - Chemn, 5 tab. 160 fig. 1509. 3 St.

$1224 \ldots-\beta-\beta$ - 1 St.

1225...2 S. Mangos, Die Mangos-Nadel. Gm. Murex molluccanus, sp. 151. Mart. 4 tab. 157 fig. 1484, 85. Lister Conch. tab. 1021 fig. 85. 1 St.

1226...2* S. Semistriatus, Die gestreifte Nadel. Gm. Murex moluccanus, sp. 151 var. 2 St.

1227...2 ** S. Tornatus, Die gedrechselte Nadel. Gm. Murex radula, sp. 147. Favanne t. XI. fig. F. Schröter Einl. in die Conch. 1 tab. 3 fig. 6. 7 St.

1228...2 $2^{* *}$ S. Picta. Der kleine Löffel. Gm. Murex cingulatus, sp. 188. MIart. t. 157 f. 1492. I St.

1229...3 S. Aluco. Die Bastard-Pabstkrone. Gm. Murex aluco, sp. 134. Mart. 4 tab. 155 fig. 1473-77. 5 St.

1230...4 S. Bubo. Die ausgelinobbelte Nadel. Gmel. Murex aluco, sp. 134 vas. 1 St.

1231...5 S. Clava Herculis. Die Herkules-Keule. Gm. Murex aluco, sp. $134 \gamma$. Chemn. 10 t. 162 f. 1548, 49. 2 St.

1232...6 S. Muricatus. Die rauhe See-Nadel. Gnel. Murex aluco, sp. 134. Mart. 4 tab. 156 fig. 1475. \& St.

1233,..7 S. Denticulatus. Die gezïlnnelte Nadel. Gm. Murex aluco, sp. 134. Mart. 4 t. 155 fig. 1477 . S St.

$1234 \ldots 8$ S. Granatius. Die granulirte Nadel. 2 St.

9) $1235 \ldots-\infty-1$ St. 
1236...9 S. Sulcatus. Die geribte Nadel. ? St. 1237...10 S. Hexagonus. Die vieleckigre Nadel. Gm. Murex - Chexagonus, sp. 163. Chemn. 10 tab. 162 fig. 1554, 55. 1 St.

2. Rostro elevato. ITit erhabener Schnautze. 1238...11 S. Nasutus. Die weisse Schneu-Penne. 2 St. 1239...12 S. Vertagus. Die weisse gefaltene Schneu-Penne. Gm. Murex vertagus, sp. 133. Mart. 4 tab. 157 fig. 1480. 3 St.

$1240 \ldots-\alpha-1$ - 4 St.

1241...13 S. Carminatus, Die bunte Schnen-Penne. Gm. Mun rex vertagus, sp. 133 ß. Mart. 4 tab. 157 fig. 1483. 3 Stück.

1242...14 S. Fasciatus. Die gebandete Schneu-Penne. Gmel. Murex vertagus, sp. 133 ß. Favanne tab. 39 fig. C. 15. 1 St.

1243...15 S. Guttatus. Die gefleckte Schneu-Penne. Gm.!Murex rertagus, sp. 133 var. 1 St.

1244...16 S, Granwlatus., Die kornigte Schneu-Penne. Gmel. Murex granulatus, sp. 149. Mart. 4 tab. 157 fig. 1482. 2 St.

1245...17 S. Cancellatus. Die gegitterte Schneu-Penne. Gm. Murex granulatis, sp. 149. 1 St.

1246...18 S. Coronatus. Die gekrönte See-Nadel, Brandpenne, Gn. IMurex aluco, sp. 134: Mart. 4 tab. 156 fig. 147.8, 79. 8 St.

\section{Lade 5 i.}

1247..19 S. Muricatus. Die ungeschliffene See-Nadel. Gm. Murex sinensis, sp. 54. 'Mart. 4 tab. 157 fig. 1489 . 8 Stïck.

1248...20 S. Annulatus. Die geringelte See-Nadel. Mart. 4 tab. 157 fig. 1486. Murex. Schröters Einleit, in die Concli. 1 p. 559 n. 41. 1 St.

3. Rostro truncato. Mit abgestumpfter Schnautze. 1249...21 S. Reticulatus. Die stachligte See-Nadel. Gm. mue rex sinensis, sp. 54 var. 5 St:

1250...22 S. Testaceus. Die șchächigte See-Nadel. Gm. Murex sordidus, sp. 13\%. 2 St. 
1251_..23 S. Pellucidus. Die durchsichtige See-Nadel. Gmel. Murex sinensis, sp. 54 var. 2 St.

1252...24 S. Perlatus. Die geperlte See-Nadel. Gmel. Murex sinensis, sp. 54 var. 1 St.

1253...25 S. Ventricosus. Die bäuchigte See-Nadel. Gm. Mulrex sordidus, sp. 13\%. 1 St.

1254...26 S. Varicosus. Die warzigte See-Nadel. Gm. Murex sordidus, sp. 137. Mart. 4 tab. 157 fig. 1490. 2 St.

1255...27 S. Irregularis. Die buntschäckigte See-Nadel. Gm. Murex sinensis, sp. 137. 1 St.

$1256 \ldots 27$ * S. Fossilis. Die gefurchte See Nadel. 1 St. $1257 \ldots 27^{* *}$ S. Rosea. Die rosenrothe See-Nadel. 2 St. $1258 \ldots 27^{* * *}$ S. Brunea. Die braune See-Nadel. 1 St.

1258a..28 S. Boletus. Die Morchel. Gm. Buccinum phallus, sp. 146. Mart. 4 t. 155 fig. 1464, 65. Schröt. FlussConch. p. 401 n. 194. 5 St.

1259...28* ! S. Tortilis. Die ausgedrechselte See-Nadel. I St.

4. Rostro subtruncato. Mit verschnittener Schnautze.

1260...29 S. Spinosus. Die Stachel-Nadel. 3 St.

1261...30 S. Turritus. Die gethürmte See-Nadel. Gm. Murex fluviatilis, sp. 141. Lister tab. 122 fig. 18 \& 20. Favanne 39 fig. C. $6.2 \mathrm{St}$.

1262...31 S. Nodosus. Die knotigte See-Nadel. 1 St.

1263...32 S. Pyramidalis. Die Pyramide. Gm. Strombus aculeatus, sp. 44 . Chemn. 9 t. 136 f. $126 \%, 68$. 4 St.

1264...33 S. Babylonicus. Die babilonische See-Nadel. 1 St.

5. Rostro obsoleto. Mit kurzer Schnautze.

1265 ...34 S. Obsusus. Die stumpfe See-Nadel. Gm. Strombus auritus, sp. 45. Favamne t. 39 fig. C. 19. 3 St.

1266...35 S. Canalicularis. Die Knopf-Nadel. Gm. Strombus lividus, sp. 49. Chemn. 9 t. 136 f. 1269, 70. 3 St.

$1257 \ldots 36$ S. Porcellanus. Gm. Strombus auritus, sp. 43. Lister Conch. tab. 121 fig. 16.6 St.

$1268 . . .36$ a. a - - Chemn. 9 t. 136 f. 1265, 66. 3 St.

*1269..37 !! S. Discoideus. Die Scheiben-Nadel. Gm. Murex sulcus, sp. 139. Selhr selten.) Schröters Einl. in die Concl. 1 p. 561 11. 47. Favanne tab. LL. fig. K. Lister tab. 120 fig. 15. 1 St. 


\section{Lunatica. Das Knobbelhorn, oder die Mond-Schnecke.}

1270... I L. Maxima. Das grosse Knobbelhorn. Gm. Turbo olearius, sp. 17. 1 St.

1271...2 L. Viriduna. Das grüne schäckigte Knobbelhorn. Gm. Turbo olearius. sp. 17 var. $\%$ Chemn. 5 tab. 178 fig. $1773,74.1$ St.

-...- - - 2 St. durchschnitten.

1272...3 L. Undulata. Das wellenförmige Knobbellıorn. Gm. Turbo marmoratus, sp. 15 . Chemn. 5 tab. 181 fig. $1775,76.1$ St.

1273...4 L. Marmorata. Das Schlangenfell. Gm. Turbo maxmoratus, sp. 15. 5 St.

$1274 \ldots-\infty-1 \mathrm{St}_{\mathrm{t}}$

$1275 . . .5 *-2$ Stück dito, worauf Blumen sehr schön geschnitten.

1276...5** L. Imperialis. Der kaiserliche Silbermund. Gm. Turbo imperialis, sp. 20. Chemn. 5 t. 180 fig. 1790. 1 Stück.

1277...6 I. Siamea. Der gedörnete Silbermund. Gm. Turbo cornutus, sp. 18. Chemn. 5 t. 179 f. 1779, 80. 6 St。

\section{Lade 55.}

1278...7 I. Chrysostomo. Der Goldmund. Gm. Turbo chrysostomus, sp. 10, Chemn. 5 tab. 778 f. 1765 . Knorr Vergn. 2 tab. 14 fig. 2 \& 5 tab. 13 fig. 3. 3 St.' 1279...8 L. Chrysostoma. $\propto-5$ St, $1280 . . .-\beta-2$ St. 1281...8 L. Citrina. Der citrongelbe Goldinund. Gm. Tuibo chrysostomus, sp. 10. 1 St.

1282... . I. Spinosa. Der stachlichte Goldmund. Gm. Turbo spinosus, sp. 24. Chemn. 5 t. 181 f. 179\%. St. 283...10 L. Granulata. Der granulirte Silbermund. Gmel. Turbo crenulatus, sp. 29. Chemn. 5 tab. $182 \mathrm{fig}$. 1811, 12. 2 St.

284...11 I. Tuberculata. Der warzigte Silbermund. Gmel. Turbo Moltkianus, sp. 26. Chemn. 5 tab. 181 fig. 1799, 1800. 2 St. 
1285...12 L. Argyrostowa Der gereifte Silbermund, Geve tab. 17 fig. 160,3 St.

1286...13 L. Argentata. Dex glatte Silbermund. Gm. Turbo canaliculatus, sp. 22. Chemu. 5 tab. 181 fir. 1794. 1 Stück.

1287... 14 L. Maculata. Der.. geflechte Silbermund. Geve tab. 17 fig. 159 a. b. \& 161.5 St.

1288...15 L. Taitensis. Der otaheitische Silbermund. \& St. (1 St. abgezogen.)

1289...16 L. Vericulum. Die schiwarze Bürste. Gmel. Turbo serosus, sp. 23. Chemn. 5 tab. 181 fig. 1795, 96. 2 St. 1290...17 !" L. Brunea. Dér schwarzbraune Silbermund. Gm. Turbo argyrostomus, sp. 41. Chemri. 5 tab. 177 fir. 176́4. 1 St. Schön.

$1291 \ldots$ - $\%$ - 2 St.

1292... 18 L. Picta. Der buntschäckigte: Silbermund. Gnel. Turbo argyrostomus, sp. 41. Chemil. 5 tab. 177 fig. 1763. 1 St.

1293...19 L. Squamosa. Der schuppigte Silbermund. Gmel. Turbo argyrostomis, sp. 41 . Chemn. 5 tab. 177 fig. 1764. 5 St. wovon 1 mit Deckel.

1294...20 L. Obscura. Der dunkelbranne Nassauer. Gmel. Turbo petholatas, sp. 8. Chemn. 5 tab. 183 fig. 1830, 31. 1 St.

*1294..21 ! L. Marmorata. Der schön marmorirte Nassaner. Gm. Turbo petholatus, sp. 8. Viel ühniliches von fig. 203 tab. 20 im Geve, nur brauner. 1 St.

1295...22 L. Porphyrid. Der Porphir-Nassauer, Gm. Turbo petholatus, sp. 8. Chemn. 5 t. 184 f. 1838 I St.

1296...23 L. Cingulata: Der gegürtelte Nassauer. Gm. Tutrbo petholatus, sp. 8. Chemn. 5 t. 183 f. 1832. 2 St. *1297...24! L. Sericea. Der Kleine schöne Nassauer. Gmel. Turbo petholatus, sp. 8. $1 \mathrm{St}$.

1298...25 L. Denigrata. Der schwärzliche Nassauer. Gmel. T. petholatus; sp.8. Chemin. 5 tab. 183 f. 1850, 31. 1 St. 1299...26 L. Petholata. Der glatte baindirte und marmorirte Nassauer. Gm. Turbo petholatus, sp. 8. 1 St.

$1300 \ldots$ - $\alpha-$ Chemn. 5 t. 183 f. 1828, 29. $13 \mathrm{St}$.

$1301 . . .-\beta-4$ St

$1302 . . . \gamma-\gamma-9 \mathrm{St}$

$1303 \ldots-\delta-$ - Chemn. 5 t. 183 f. 1831, 35.4 St. 


\section{Lade 56.}

\section{Lunella. Die Mond-Schnecke.}

\section{Spira elevata.}

1304...1 L. Viridana. Die stachligte Mondschnecke. Gmel. Turbo coronata, sp. 21. Chemn. 5 tab. 180 fig. 1791 -93. $2 \mathrm{st}$.

$1305 \ldots-\infty-1$ St.

1306...2 I. Moniliformis. Die knotige Mondschnecke. Gm. Turbo granulatus, sp. 51 ß. Chemn. 5 tab. 176 fig. 1745. 3 St.

1307...S L. Appendiculata. Die gestachelte Mondschnecke. Chemn. 5 tab. 171 fig. 1665.2 St.

1308... L Lidaroides. Die gerunzelte Mondschnecke.'Ch. 5 tab. 165 fig. 1567, 68. 2 St.

1309...5 L. Anomala. Die wulstige Mondschneche. Gmel. Turbo cidaris, sp. 34. viridis. Chemn. 5 tab. 184 fig. 1842, 43. 2 St.

1310...6 L. Moslemica. Die Emirs-Mondschnecke. Gmel. Turbo cidaris, sp. 34 . Cliemn. 5 tab. 184 fig. 1842. \% Stück,

\section{Spira contigua.}

1311...7 L. Picta. Die buntschächigte Monảschnecke; Gmel. Turbo versicolor. sp. 43. Chemn. 5 tab. 176 fig. 1740, 41. 3 St.

1512...8 L. Striata. Die gebandéte Mondschnecke. $2 \mathrm{St}_{\text {. }}$ 1313...8* L. Pulverata. Die gepuderte Mondschnecke. I St. 1314...9 L. Cruenta. Die mit Blut geflechte Mondschnecke. 2 Stück.

1315...10 L. Iriteola. Die gelbe Mondschnecke. Gm. Turbo neritoides, sp. 2. Clemn. 5 t. 185 f. 1854, 55. 2 St. 1315a.. - a - - Chemn. 5 t. 185 f. 1854 fig. 9, 10. 2 St. 1316...11 L. Neritoides. Die neritenförmige Mondschnecke. Gm. Turbo obtusatus, sp. 1. Chemn. 5 tab. 185 f. $/ 17$ 1854 fig. e. 2 St.

1317...12 L. Vitellata. Die weiss - und gelbgebandete Mondschnecke. Gm. Turbo neritoides, sp. 2. Chemn.5 tab. 185 f. 1854 fig. 3. 1 Sto 
1318... Is L. Radula. Die scharfe Mondschnecke. Gm. Nie. rita radula, sp. 2\%. Chemn. 5 tab. 190 fig. 1945, 47. 3 Stiick.

Lade 57.

\section{Pythia. Die Zauber-Schnecke.}

1319...1 P. Helicina. Die Hexe. Gm. Helix scarabous, sp. 1. Chemn. 9 tab. 136 fig. 1249, 50. Know Vergn. 6 tab. 19 fig. 2, 3. 6 St.

\section{Ellobium. Das Midas-Ohr.}

\section{Cylindracea.}

1320...1 E. Midae. Das grosse Midas-Ohr. Gm. Voluta auris midae, sp. 1. abgeschliffen. Mart. 2 tab. 43 fig. 436, 3\%. 1 St. und 2 St. abgezogen.

1321...2 E. Ceramense. Das grosse dïnnschalige Midas-Ohr. Gm. Voluta auris midae, sp. 1. Miart. 2 tab. 43 fig. 438. 1 St.

1922...8 E. Tumidum. Das diclibauchigte Midas-Ohr. Gm, Voluta auris midae, sp. $1.1 \mathrm{St}$.

1323...4 E. Labrasum. Das kleine Midas-Ohr. Gm. Voluta auris judae; sp. 10. Mart. 2 t. 44 f. $449,50.3$ St.

1324...5 E. Subtile. Das kleiue diinnschalige Midas-Ohr. Gm. Voluta auris judaé, sp. 10. INart. 2 tab. A4 fig. 451. 1 Stück.

1225...6 E. Inflammatum. Das gebandete Midas-Ohr. Gmel. Voluta coffea, sp. 15. Lister t. 834 fig. 59. Chemn. 9 tab. 121 fig. 1043,44 . 1 St.

1326...7 E. Barbadense. Das Midas-Olir von Barbados. Gm. Voluta coffea, sp. 15. Mart. 2 t. 43 f. $445.1 \mathrm{St}$.

\section{Buccinoidece.}

"1327..8 ! E. Australe: Das neuseelïndische Midas-Olır. Gm. Voluta auris Malchi, sp. 11, selten. Chemn. 9 tab. 121 fig. 1039, 40. 1 St.

1328...9 E. Flammeum. Das bunte Midas-Ohr. Gm. Voluta glabra, sp. 8. Mart 2,t. 43 f. 447, 48. 4 St.

1329...10 E. Ceriona. Das schneeweisse Midas-Ohr. Gm. Helix labiosa, sp. 101. Chemn. 9 t. 135 f. 1234. 2 St. 
1330...11 E. Virgincum. Das blasrothe Midas-Oli1. Gm. Helix pudica, sp. 102. Chemn. 9 tab. 121, fig. 1042. 1 Stück.

\section{Helix. Die Schraube.}

1331... H H. Hepatica. Die braune Schraube. Gm. Bulla fas. ciati, sp. 25. 1 St.

1332...2 Ir. Testa-ovi. Die weisse Schraube, Gmel. Bulla fasciata, sp. 25. 2 St.

1333...3 H. Fasciata. Die Staten.Flagge. Gm. Bulla fasciata, sp. 25. Chemn. t. 117 f. 1004-1006. 4 St.

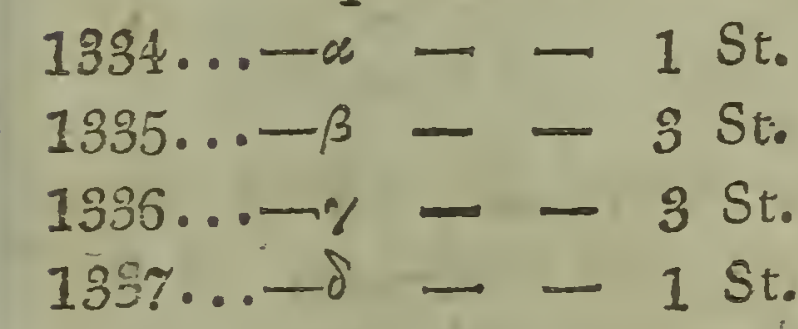

Is38...4 H. Oblonga. Bas falsche Midas-Ohr, Rosenmund.

Gm. Helix oblonga, sp. 87. Chemn. tab. 119 fig. 1022, 23. 3 St.

1339...5 H. Globulus. Das kleine dünnschalige falsche Midas= Ohr. 2 St.

*1340.6 H. Fulica. Dis binte Henne, selten. 1. St.

1341...7 H. Ellobioides. Das gestreckte Midas-Olir. $1 \mathrm{St}$.

13\{2...8 H. Rallus. Der bunte Halin. Gm. Bulla Zebra, sp.

31. Chemn. 9 tab. 118 fig. $1015,16 \delta .5$ St.

1313...9 H. Tumida. Die lileine bäuchigte Schraube. Gmel. Bulla detrita, sp. 139. 1 St.

1941...10 II. Elongata. Die lang, gestrechte Schraube. Gm. Helix detrita, sp. 139. Chemn. 9 tab. 134 f. 1225 a. 1 Stück.

1345...11 H. Ligata. Die gebandete Sclraube. I St.

1316...12 H. Brunnea. Die brannlich gebandete Schraube, 1 Stück.

1347...13 H. Albida. Die schmutzig weisse Schraube, 1 St. 1348...14 H. Carinata. Die buntschäckige Schraube. $1 \mathrm{St}$. $1349 . . .-\%$ - 1 St.

1350...15 H. Detrita. Die abgeschliffene Schraube, Gmel。 Helix detrita, sp. 139. Chemn, 9 tab. 134 fig. lit. c. d. 4 St.

$1351 \ldots-\alpha-2$ St.

${ }^{*} 1352 . .-\beta-$ - links gewunden, selten, 1 St: 
*1353..16 H. Biliosa. Die vielfarbige links Schraube. Gmel. Helix perversa, sp. $94 \%$ selten, Chemn. 9 t. 110 fig. 932, 33. 2 St.

1354...- - - - Chemn. 9 tab. 110 fig. 936, 3\%. 1 St.

1355...17 H. Citrina. Die citronengelbe rechte Schraube. 1 Stück.

1556...18 H. Dextra. Die citronenfarbene Schraube. Gmel. Helix dextra, sp. 95. Chemn. 9 tab. 134 fig. 1210, 11. $2 \mathrm{St}$.

$1357 \ldots-\alpha-1$ - -1 St.

*1358..19 ! H. Perversa. Die linksgewundene schwefelgelbe Schraube. Gm. Helix perversa, sp. 94, selten. Ch. 9 tab. 110 fig. 934, 35. 1 St.

1559... - - - - ganz weisse. 2 St.

1360...20 H. Inversa. Die streifigte Schraube. Gmel. Helix inversa, sp. 97. Chemn. 9 t. 110 f. 925, 20. 6 St.

$1361 \ldots-\alpha-1$ St.

*1362..21 If. Testacea. Die castanienbraune Schraube. Gmel. Helix inversa, sp. 97 var. sehr selten.' I St.

*1363.:22 H. Laeva. Die umwundene Schraube. Gm. Helix laeva, sp. 100, selten. Chemn. 9 tab. 111 fig. 946, 47. 2 St.

*1364..23 H. Interrupta. Die unterbrochene Schraube. Gm. Helix contraria, sp. 99, selten. Chemn. 9 tab. 111 fig. 938, 39. 1 St.

*1365..24 H. Speciosa. Die vorzüglich schöne Schraube. Gm. Helix contraria, sp. 99. selten. 1 St.

*1366..25 Fr. Listeri. Die Listersche Schranbe. Gm. Helix columna, sp. 122, äusserst selten. Lister Conch. tab. 38 fig. 37 \& 39. Chemn. 9 t. 112 f. $954,55.1$ St.

1267...26 IH. Ustilago. Die unächte gestreckte Schraube. Gm, Helix flammea, sp. 88. Cliemn. 9 tab. 119 fig. 1024, 25. 1 St.

*1368..27 H. Phasianus. Der Phasan. Gm. Buccinum australe sp: 173, selten. Chemn. 9 t. 120 f. 1053 , 34. 2 St.

1369...27* H. Variegata. Die gesprenkelte Schraube. 2 St. 1370...28 H. Pulverilenta. Die gepuderte Schraube. $1 \mathrm{St}_{\mathrm{i}}$

1371...29 H. Stagnalis. Die düme Fluss-Schraube, Spitzhorn, Gm. Helix stagnalis, sp. 128. Chemn. 9 tib. 155 fig. 1237, 38. 5 St. 
1372...30 H. Fragilis. Die durchsichtige Fluss.Schraube. Gm. Helix stagnalis, sp. 128. $2 \mathrm{St}$.

1573...3i H. Palustris. Die braunmündige Schraube. Gmel. Helix stagnalis, sp. 128. Mart. 1239, 40. 6 St.

1374...32 H. Vivipara. Die stumpfe Schraube. Gmel. Helix vivipara, sp. 105 ß.' Chemn. 9 tab. 132 fig. 1182, 83. 5 St.

1375...33 H. Angaria. Die Hornschraube. Gm. Turbo lincina, sp. 71. Chemn. 9 tab. 123 fig. 1060 a. 1 St. 1376...34 H. Buccinulum. Die kleine gelbe Schraube. Gmel. Turbo lincina, sp. 71. Mart. 1060 b. c. 2 St.

\section{Thiara. Die dreifache Irrone.}

137\%...1 T. Aniarula. Die Rivier Pabst-Krone. Gm. Helix amarula, sp. 126. Chemn. 9 tab. 134 fig. 1218, 19. Rumpf tab. 33 fig. F.F. 7 St. 1 St. defect.

1378...2 T. Cancellata. Die gegitterte Rivier Pabst-Krone. Gm. Helix amarula, sp. $126 \beta$. Chemn. 9 tab. 134 fig. 1220, 21. 1 St.

*1379...3 T. Eximia. Die lang gertreckte Rivier Pabst-Krone, selrr selten. 1 St.

*1380..4 T. Praestans. Die gelbbraune Rivier Pabst-Krone, sehr selten. i St.

1381...5 T. Minuta. Die kleine Pabst-Krone. 3 St.

\section{Pupa. Die Puppe.}

1382... 1 P. Solidula. Die dickschalige Puppe. Gmel. Voluta flammea, sp. 2. Mart. 2 tab. 43 fig. 439. Lister tab. 814 fig. 24. 1 St.

1383...2 P. Frisebla. Die gebandete Puppe. Gm. Voluta so. lidula, sp. 13. Chemn. 10 t. 149 f. 1405. 2 St.

\section{Oleacina. Die Olive.}

1384...1 O. Volutata. Die Olive. Gm. Bulla voluta, sp. 49. Chemn. 9 tab. 117 fig. 1009, 10. I St. $1385 \ldots-\infty-5$ St. diverse. 


\section{Lade 58}

\section{Ampulla. Der Wasserkrug.}

1386...1 A. Achatina. Das rothe Rebhuhn. Gm. Bulla acha. tina, sp. 32 ß. Chemn. 9 t. 118 f. 1012, 13. 5 St.

$1387 \ldots-\%$ - $2 \mathrm{St}$.

1388...2 A. Flammea. Der geflammte Wasserkrug. Gm. Bulla achatina, sp. 32. 2 St.

1389..3 ! A. Bombarda. Der kugelförmige Wasserkrug. Gm. Bulla achatina, sp. 32. 1 St.

1390.. .4 A. Lacteae. Der milchweisse Wasserkrug. Gmel。 Bulla achatina, sp 32. 1 St.

1391...5 A. Purpurea. Der purpurfarbne Wasserkrug. Gm: Bulla achatina, sp. 32. Mart. 1017, 18. 3 St.

1592...6 A. Trambeul. Der geglättete Wasserkrug. Gm. Bulla Zebra, sp. 31. Mart. 1024, 25. Lister 578 fig. 33. 9 Stück.

1393...7 A. Zebra. Der grosse Kapsche Esel- Gmel. Bulla Zebra. sp. $31 \delta$. Chemn. 9 tab. 118 f. 1014. 7 St.

$1394 \ldots-\alpha-1 \mathrm{St}$.

1395...8 A. Quagga. Der gestreckte Zebra. Gm. Bulla Ze. bra, sp: 31. 1 St.

*1395..9 !! A. Priamus. Der Priamus. Gm. Bulla Stercus pulicum, sp. 45. Chemn. 9 t. 120 fig. 1026, 2\%. 1 Sto

\section{Lade 59.}

\section{Buccinum, Das Kink-Horn.}

1997...1 B. Undatum. Das wellenförmige Khink-Horn. Gm, Buccinum undatum, sp. 93. 2 St.

$1398 \ldots-\omega-2 \mathrm{St}_{\mathrm{t}}$

$1399 . . .-\beta-1 \mathrm{St}$.

1400...-y - Mart. 4 t. 126 f. 1206-10. 1 St.

1401...2 B. Tumidum. Das bauchige Kink-Hom. 2 St.

*1402..3 ! B. Elongatum. Das dünne aufgeblasene Kink-Hotn。 mit 9 Windungen, selten. 1 St.

1403...4 B. Anglicum. Das englische Kink-Horn. Gm. Buccinum vulgatum, sp. 120. Mait. 4 tab. 124 fig. 1162, 69. $14 \mathrm{St}$. 
1404...5. B. Porcatnm. Das gefurchte Kink-Horn. Gm. Buccinum vulgatum, sp. 120 ß. Mart. 4 tab. 124 fig. $1165,66.2 \mathrm{St}$.

1405...5* B. Dorsatum. Der Schildträger. Gm. Buccinum vulgatum, sp. 120. Mart. 4 t. 125 f. 1194, 95. S St. 1406... - - -1 St. $1407 \ldots-\beta-\beta-1$ St.

1408...6 B. Quadridenticulatum. Das vierzähnige Kink-Iorn.

Gm. Buccinum vulgatum, sp. 120 var. 1 St. 1409...6* B. Politunt. Das glatte Kink-Horn. Gm. Buccinum vulgatum, sp. 120. 1 St.

1410...7 B. Dubium. Das gefaltene Kink-Horn. eod. 1 St. 1411...7 * B. Cassiatum. Das Helmförmige Kinl-Horn. eod. 1 Stück.

$1412 \ldots-\alpha-1 \mathrm{St}$.

1413...8 B. Chrysostomzum. Das kleine gegitterte hink-Horn. Lister 966 fig. 21 eod. ' 1 St.

1414...9 B. Granulare. Das körnigte Kink-Horn, eod. I St. 1415...10 B. Striatum. Das gestreifte Kink-Horn 1 St. $1416 \ldots-\alpha-2 s t$.

*1417..11 B. Contortum. Die linksgedrehte Harfe, selir sel. ten, 1 St.

1418...12 B. Glaciale. Das Kink-Horn des Eismeers. Gm. Buccinum glaciale, sp. 92. Chemn. 10 tab. 152 fig. 1446,47 . S St.

1419...13 B. Islandicum. Das isländische Kink-Horn. Gmel, Buccinum indicum, sp. 114. Mart. 4 tab. 123 fig. 1138, 39. $2 \mathrm{St}_{*}^{\prime}$

1420...14 B. Lineatum. Das liniirte Kink-Horn. Gm. Buccinum glans, sp. 41. Mart. 6 tab. 125 fig. 1196, 97. 4 Stïck.

1421...14* B. Faba. Die weisse Bolne. 1 St.

'1422..15!! B. Turgidum. Das vielpunctirte Kink-Horn, Gm. Buccirum turgidum, sp. 184. Chemn. 10 tab. 154 fig. 1475, 76. Martyn. univ. Conch. 2 tab. 49 d. selten. $1 \mathrm{St}$.

1423...16 B. Flammigerum. Das gezackte Kink-Horn. 1 St。 [424...17 B. Cereum. Das Wachsgelbe Kink.Horn. 1 St. '1425..17* !! B. Fenestratum. Das grosse gegitterte Kink Horn, sehr selten. 1 St. 
1426...18 B. Cinctum. Das hollkellige Fink-Horn. Gmel. Buccinum porcatum, sp. 105. Niart. 4 tab. 126 fig. 1213, 14. Knorr 6 t. 33 f. 3. 2 St.

1427...19 B. Britanicum. Das brittische Khink-Horn. Gmel. Buccinum ánglicum, sp. 104. Mart. 4 tab. 126 fig. 1212. 1 St.

*1428..19* B. Scalare. Die Treppe. Gm. Buccinum anglicum, sp. 104, selten. I St.

1429...20 B. Excavatum. Das ausgelıobelte Kink-Horn. Gn. Buccinum anglicum, sp. 104. 4 St.

$1430 \ldots-\omega-2 S t$.

$1431 \ldots-\beta-1$ St.

*1432..20* B. In pressum. Das eingedrückte Kink-Horn. 1 St.

1433...21 B. Rufescens. Das buntschächigte Kink-Horn. Gm. Buccinum porcatum, sp. 105. 2 St.

1434...22 B. Inflatum. Das weissgekehlte Kink-Horn. 1 St.

*1435..23 ! B. Lamellosum. Das blättrige braune Kink-Horn. Murex Australasiae, aus der Südsee. $2 \mathrm{St}$.

*1436. - $-\infty-$ - sehr selten. 2 St.

1437...24 B. Papillosum. Der Reisbrei. Gm. Buccinum papillosum, sp. 40. 'Mart. 4 t. 125 fig. 1204, 5. Yinour 2 tab. 27 fig. 2. 2 St.

113 1438...25 B. Punctulatum. Das punctirte Kink-Horn. I St. 1439...26 B. Diaphanum. Das klare Kink-Horn. Gm. Buccinum textum, sp. 102. Mart. 4 tab. 125 fig. 1201, 2. i Stück.

1440...27 B. Camellinum. Das gerippte Fink-Hom. I St.

1441...28 B. Senticosum. Das Diestel-Horn. Gm. Murex senticosus, sp. 49. Mart.4.tab. 155 fig. 1466, 67. Fnort 4 tab. 23 fig. 4, 5. 5 St.

1442...29 B. Cassidoideum. Das gegürtẹlte Kink-Horn. 1 St. $1443 \ldots 30$ B. Lamboides. Das ausgeschnittene Kink-Horm. 2 Stück.

$1444 \ldots 31$ B. Laevigatum. Das geglättete Kink-Hom. Gmel. Buccinum laevigatum, sp. 129. Mart. 4 tab. 125 fig. 1214, 16. 2 St.

$1445 \ldots 32$ B. Porphoroides. Das zugespitzte Kink-Horn. 5 St. $1446 \ldots 33$ B. Obtusum. Das stumpfe Kink-Horn. 2 St.

1417...34 B. Cingulatum. Die kleine zeylonsche Dattel. Mart. 3 fig. 564. 3 St. 
1448...35 B. Glabratum. Die zeylonsche Dattel. Gm. Buccinum glabratum, sp. 81. Lister t. 974 f. 29. Mart. 4 tab. 122 fig. 1117. 6 St.

$1449 . . .-\alpha--1 \mathrm{St}$.

$1450 \ldots-\beta--1$ St.

$1451 \ldots-y-2$ - $2 t$.

1452...36 B. Spiratum. Die genabelte Fischräuse: Gm. Buc: cinum spiratum, sp. 70. Mart. 4 tab. 122 fig. 1118. Knorr 2 t. 6 f. 5 \& 3 t. 3 f. 4. 10 St.

1453...s7 B. Giratum. Die gezähnelte Fischreuse. Gm. Buc. cinum glabratum, sp. $81 \beta$. Mart. 4 tab. 122 fig. 1119. 5 St.

1454...38 B. Maculosum. Die geflechte Fischreuse. Gm. Buccinum spiratum, sp. $70 \beta$. Mart. \& t. 122 fig. 1120 , 21. 3 St.

B. Rude. Das plumpe Kink-Hoin. Gm. Buccinum spiratum, sp. 70. 3 St. 1456...40 B. Tuberculatum. Das geknobbelte Kink.Horn. $1 \mathrm{St}$. $1457 . .-\infty-1 S_{t}$

\section{Lade 60.}

\section{Neptunea. Das Neptunus-Horn.}

1458...1 N: Despecta. Das isländische Neptunus-Horn. Gm. Murex antiquus, sp. 7ร. Mart. 4 tab. 138 fig. 1895. 6 Stück.

1459...2 N. Neglecta. Das nordische Neptunus-Horn. Gmel; Murex antiquus, sp. 73 var. Mart. 4 tab. 138 fig. 1295. 2 St.

1460...3 N. Limbata. Das gesäumte nordische Neptunus-Horn. Gm. Nurex antiquis, sp. 33. 3 St.

- ...- - - Mart. 4 t. 139 t. 1291. 3 St.

1461...4 N. Antiqua. Das fein gegitterte nordische Neptunus. Hom. Gm. Murex antiquus, sp. 73. Mart. 4 tab. 138 fig. $1 \times 94.8$ St.

1462..5! N. Cointraria. Das linksgewundene noraische N'eptunus-Horn. Gm. Murex contrarius, sp. 15\%. Mart. 9 tab. 105 fig. 894, 95: Regenfuss Conch. 2 tab. 4 fig. 36. 1 St:

*1463..6! N. Perversa. Das linksgewundene dickschalige nor. dische Neptunus.FIorn。 1 St. 
1461...7 N. Ponderosa. Das braunangelaufene nordische Neptunus-Horn. 1 St.

*1465..8! N. Corona Mexicana. Die Mexicanische Krone. Gm. Murex corona, sp. 161, äusserst selten. Chemn. 10 tab. 161 fig. 1520, 27, Davil. catal. raris. 1 tab. 9 fig. A. 1 St.

*1466..9 N. Clathrus. Das blättrige nordische Neptunus-Horn. Selten. Kammerer tab. 9 fig. 2. 2 St.

${ }^{*} 1467 \ldots-\infty-$ - Sehr selten. 3 St.

*1468..10 ! N. Foliacea. Der Granat- $\Lambda$ pfel. Gm. Murex magelanicus, sp. 80. Mart. 4 tab. 139 fig. 1297. Knorr 4 tab. 30 fig. 2. 4 St.

*1469..10* N. Muricina. Das fein geknobbelte nordisclie Neptunus-Fiorn. $1 \mathrm{St}$.

*1470..10 ** N. Canaliculata. Das ausgedrehte nordische Neptunus-Horn. 1 St.

1471...11 N. Cancellata. Das Gitterwerk. 1 St.

1472...12 N. Reticulata. Das Netz. 2 St.

*1473..13 ! N. Magellanica. Die gegitterte Sclinecke aus NeuSeeland. Gm. Murex magelanicus, sp. 80 $\beta$. selten. Chemn. 10 tab. 164 fig. 1570. 1 St.

1474...14 N. Varicosa. Die wulstige Schnecke. Gm. Nurex rubecula, sp. 35. Chemn. 10 táb. 162 fig. 1546, 47. s Stück.

$1475 \ldots 14 *$ N. Fossilis. Die ausgegrabene Schnecke. $1 S_{i}$.

1476...15 N. Pusilla. Das kleine weisse Gitterwerk. Gmel. Buccinum niveum, sp. 112. Mart. 4 tab. 122 fig. 1122, 23. 3 St.

$147 \%$..16 N. Doliata. Das geringelte Neptunus-Horn. Gm. Buccinum caudatum, sp. $6 \beta$. Lister Concl. tab. 940 fig. 35. 4 St.

116 1478...17 N. Nasata. Die zugespitzte Schnecke. 2 St.

\section{Colus. Der'Spinnrocken.}

*1479..1! C. Tulipa. Die Tulpe. Gm. Murex tulipa, sp.9I. Selten. Lister Conch. 910 fig. 1. Tour d'Auvergn. tab. 1003. $1 S t$. 
1480...2 C. Achatinus Der Agat-Spinnrocken. Gm, Murex tulipa, sp. 91. Mart. 4 tab. 136 fig. 1288, 89. 7 St.

$1481 \ldots-\infty-3 \mathrm{St}$.

$1482 \ldots-\beta$ - - Mart. 4 tab. 136 fig. 1287. 2 St.

\section{Lade 61.}

1483...3 C. Marmoratus. Der Marmorirte Spinnrocken. Gm. Murex tulipa, sp. 91. Mart. 4 t. 156 f. 1286. 8 St. 1484...4 C. Islandicus. Der isländische Spinnrocken. Gmel. Murex islandicus, sp. 110. Mart. 4 tab. 141 f. 1312, 13. 5 St.

1485..5 C. Albula. Der weisse durchsichtige Spinnrocken. eod. var. 3 St.

1485...6 C. Albeola. Der weissgelbe Spinnrocken, eod. var. 2 Stück.

1487...7 C. Caesia. Der bläuliche Spinnrocken. Lister Conch. tab. 914 fig. 6. 3 St.

$1488 \ldots-\alpha-1$ St.

$1489 \ldots-\beta-\beta$ - 3 St.

1490..8 C. Ficoides. Der feigenförmige Spinmrocken. Lister Conch. tab. 1028 fig. 2 A. 5 St.

\section{Fusus, Die Spindel.}

1. Columella plicata. Mit gefalteter Spindel. 1491...1 F. Trapezium. Das persische Kleid. Gm. murex tra. pezium, sp. 99. Mart. 4 t. 139 fig. 1298, 99. 8 St. 1492...- - - 2 St. auf einer Korallen. 1493...2 F. Cynara. Die Artischocke. Gm. Murex scolymus, sp. 101. Mart. A to 142 f. 1325. A St,

\section{Lade 62.}

*1491...3. F. Cydonium. Die Quitte, eod. var. selten. 1 St. 1495..4 F. Simplex. Die glatte Spindel. 4 St.

${ }^{*} 1493 . .4 *$ F. Scrupeus. Die warzige Spindel. $1 \mathrm{st}$. $1497 \ldots 5$ F. Filamentosus. Die geringelte spindel. Gm. Murex trapezium, sp. $99 \beta_{0}$ Mart. 4 tab. 140 fig. 1310 , 11. 12 Stück.

1498...6 F. Polyggonus. Die vielwinklige Spindel. Gm. Murex polygonus, sp. 109. Mart. 4 t. 140 f. 1306, 7 . Knori 5 t. 10 f. $4 \& 6$ t. 15 f. 5.5 St. 
84

1499...6* F. Polyedros. Die vielwinklige Spindel. Gm. Irurex polygonus, sp. 109. 1 St.

1500...6 ** F. Angulatus. Die wulstige Spindel. Gm. Murex polygonus, sp. 109. Mart. 4 tab. 141 fig. 1314, 15. I Stück.

$1501 \ldots 6^{* * *}$ F. Fasciatus. Die weissgebandete Spindel. Gm. Murex undulatus, sp. 173. Lister Conch. 921 f. 14 a. Chemn. 10 tab. 162 fig. 142, 43. Cab. Rudolst. p. 138 11. 5 t. 10 f. 1. 2 St.

1502...7 F. Glaucus. Die bläuliche Spindel. 4 St.

1503...8 F. Minutus. Die kleinliche Spindel. Mart. 4 tab. 141, fig. 1121, 22. 4 St.

1504...9 F. Cingulatus. Die umgürtelte Spindel. Gm. Murex Nassa, sp. 93. Mart. 4 tab. 122 fig. 1131, 32. 3 St. $1505 \ldots-\alpha$ - - Knory 6 tab. 20 fig. 7. 1 St. 1506...- $\beta$ - - Mart 4 tab. 123 fig. 1133, 34. 1 St. 1507...10 F. Tapeta-persicum. Der persische Teppich. Gm. Murex gibbulus, sp. 125 . Mart. 4 t. 140 f. 1308, 9 . Knorr 5 tab. $10 \mathrm{fig}$. 4. $4 \mathrm{St}$.

1508...11 F. Anaras. Die Ananas. Gm. Murex infundibulum, sp. 103. Lister Conch. 921 f. 14. Mart. 4 tab. 143 ving. 39 f. A. 2 St.

1509...11* F. Striatus. Die gestreifte Spindel.- Gm. Murex verrucosus, sp. 120; Mart. 4 to, 144 f. 1341. 1 St.

$1510 \ldots 11$ *.. F. Filatus. Die umwundene Spindel. I St.

1511...12 F. Craticulatus. Die Rost-Spindel. Gm. Murex craticulatus, sp. 105. Mart. 4 tab. 149 fig. 1382, 83. 5 Stück.

1512...13 F. Pulcher. Die kleine gegitterte Spindel. $1 \mathrm{St}$. 1513...13 F. Iris. Die Regenbogen-Spindel. Gmel. Murex prismaticus, sp. 17.0. Chemn. 10 t. 169 f. 1635, 36. 1 Stücls.

1514...15 F. Purpuratus, Die purpurfarbene Spindel. 3 St.

2. Columella glabra. Mit glatter Achse.

1515...16 F. Trompeta: Die Trompete. Gmel. Nurex tuba, sp. 103. Mart. 4 tab. I\$3 hg. 1333. 7 St. wovon 3 selir gross.

1516...17 F. Pyramidis. Die Pyramide. LGmel, Mur. tuba, sp. 103 var: 1 St. 
1517...18 F. Ternatanus. Die Ternatanische Spindel. Gmel. Murex ternatanus, sp. 10\%. Mart. 4 tab. 140 f. «304, 5. 2 St.

1518...- - - - Knorr 6 t. 15 f. 4 \& t. 26 f. 1. 1 St. $1519 . . .-\beta--1 S t_{\text {. }}$

\section{Lade 63.}

1520...19 F. Morio. Der gebandete Mohr. Gm. Murex mo: rio, sp. 62. Mart. 4 t. 139 f. 1300.17 St.

1521... - « - - Mart. 4 t. 159 f. 1301. 4 St.

$1522 \ldots-\beta-1 \mathrm{St}$.

$1523 \ldots-\gamma-2 \mathrm{St}$.

$1524 \ldots-\delta-\ldots-1 \mathrm{St}_{\mathrm{s}}$

1525...20 ! F. Aethiops. Der ungekerbte Mohr. Gm. Murex morio, sp. 62 var. 1 St.

1526...21 F. Pugilinus. Die gelbe gezakte Spindel, Gm. Murex vespertilio, sp. 100. Mart. 4 tab. 142 f. $132 \%$, 24. $5 \mathrm{St}$.

$1527 \ldots-\infty-2$ St.

$1528 \ldots-\beta-3$ St.

1529...22 F. Carnarius. Die doppelt gezackte Spindel. Gm. Murex vespertilio, sp. 100. Chemn. 10 tab. 164 fig. 1556, 67. 2 St.

1530...23 ! F. Deplanatus. Die ausgedrechselte Spindel, braun von Farbe ohne Binden. Gm. Murex morio, sp. 62 var. 2 St.

1531...24 F. Maroccanus. Die Marocanische Spindel. Gmel. Murex maroccensis, ta 132. Chemn. 9 tab 105 f. 896. 7 St.

$1532 \ldots-\alpha-1$ st. $_{\text {. }}$

\section{Lade 64.}

\section{Syṛinx. Die Sprütze.}

Sämmtlich vorzüglich schön.

1539...1 S. Aruana. Das Aruansche Horn. Gm. Murex aruanus, sp. 71. Eins von ausserordentlicher Grösse, das andre kleiner, aber von vorzüglicher Schönlieit. Chemn. 4 pag. 143 vign. 39 f. D. Rumpf tab. 28 fo. A. $2 \mathrm{St}$. 
1534...2 S. Longissinza. Die sehr lange Sprütze. Gm. Murex longissimus, sp. 116. Mart. \& tab. 144 fig. 1354. 2 Stück.

1535...3 S. Producta. Die ausgereckte Sprütze. Gm. Murex candidus, sp. 113. Mart. 4 t. 144 f. 1339. 1 St.

1536...4 S. Tabaccaria. Die Tobakspfeife. Gm. Murex undatus, sp. 115. Mart. 4 t. 145 f. 1343. 1 St.

I53\%...5 S. Tornata. Die gedrechselte Sprütze. Gm. Murex colus, sp. 41. Mart. 4 t. 144 f. 1342. 5 St.

1538...- - - 3 St. monströse.

1539...6 S. Maxima. Die grosse Sprütze.' Gm. Murex longissima, sp. 116 var. mit sehr scharfen Reiffen, und nicht geknobbelt. 1 St.

1540...7 S. Maculata. Die gefleckte Sprütee. Gmel. Murex ansatus, sp. 114. Mart 4 t. 144 f. 1340. 5 St.

1541...8 S. Nicobarica. Die Nicobarische Sprütze. Gm. Mnrex colus, sp. 61. Chemn. $10^{\circ}$ tab. $160^{\circ}$ fig. $1529 \%$ 2 Stück.

1542...9 S. Marmorata. Die bunte Sprütze. Gm. Murex colus, sp. 61 var. Chemn. 10 t. 160 f. 1523. 1 St.

1543...10 S. Viminalis. Die lineirte Sprütze. Gm. Murex colus, sp. 61 var. 1 St.

1544...11 S. Petiolata. Die hleine Sprütze. Gm. Murex colus, sp. 61 var. 2 St.

1545...12 S. Annulata. Die geringelte Sprutze. Gm. Murex polygonus, sp. 109 var. Mart. 4 tab. 141 fig. 1216. 1 Stück.

1546...13 S. Clathrata. Die gegitterte Sprütze. Gm. Murex polxgonus, sp. 109. Mart. 4 t. 141 f. 1916. 4 St.

1547...14 S. Buccinoidea. Die aufgeblasene Sprütze. Gmel. Murex verrucosus, sp. 120 . Mart. 4 tab. 144 fig. 1341. 2 St.

1548...15 S. Parvulus. Die warzige Sprintze. Gm. M. verrucosus, sp. 120. Nart. 4 t. 144 f. 1341. 2 St.

1549...16 S. V eirosa. Die adrichte Sprütze. Gm. Murex ver-, rucosus, sp. 120.1 St.

1550...17 S. Elongata. Die ausgereckte Sprütze. Gm. Murex Golus, sp. 61 var. 1. St.

1551...18 S. Ficoidea. Die abgesetzte Sprütze. 2 St.

1552...19 S. Foșsa. Die ausgegrabene Sprütze. Gm. M. fossi, lis, sp. 112. Mart. 4 t. 141 f. 1321, 22. 1 St. 


\section{'Tibia. Die Flöte.}

1553...1 T. Insulae Chorab. Die grosse dicke Stern-Nadel. Gm. Strombus fusus, sp. 1. Mart. 4 t. 158 f. 1495, 95. 4 St.

*1554..2 T, Ixdiarum. Die Ostindische Stern- Nadel. Gmel. Strombus fusus, sp. $1 \%$. Ausserst selten und schön. Favanne 34 fig. B. 3. Mart. 4 vign. 41 P. 34 t. 1 St. 1555...3 T. Clavus. Die ungezähnte Stern-Nadel. Gm. Strombus clavus, sp. 7. Mart. 4 t. 159 f. 1501, 2. 3 St. 1556...4 T. Fissurella. Die gespaltene Stem-Nadel. Gmel. Strombus fissurella, sp. 28. Mart. $\&$ tab. 158 fig. 1498, 99. 1 St. -...-Verkalkte 5 St.

Lade. 65.

\section{Turris. Der Thurm.}

1557...1 T. Babylonica. Der Babilonische Thurm. Gm. Mu• rex babylonus, sp. 52. Mart. 4 tab. 143 fig. 1331, 32. 7 Stück. $1558 \ldots-\infty-2$ - 2 St.

1559...2 T. Nobilis. Der geringelte Thurm. Gm. Murex ba* bylonus, sp. 52 var. 2 St.

1560...3 T. Pulchra. Der schöne Thurm. Gm. Murex babylonus, sp. 52 var. 1 St.

1561...4 T. Imperfecti. Der unvollkommene Thurm. Gmel. Murex babylonus, sp. 52 var. 2 St. 1562...5 ! T. Excelsa. Der hohe Thurm. 3 St. 1563...6 T. Javana. Der durchsichtige Thurm. Gm. Murex javanus, sp. 53. Mart. 4 t. 149 f. $133 \%$, 38. 4 St. 1564...7 T. Taxea. Der blasgelbe Thurm. Gm. Murex babylonus, sp. 52 \}. Chemn. 10 tab. 162 fig. 1550, 51. 2 Stück.

1565...s T. Indica. Der indianische Thurm. Gm. Murex babylonus, sp. 52 8. Mart. 4 tab. 144 fig. 1345, 46. 4 St.

1556...9 T. Gothica. Der gothische Thurm. Gm. Murex babylonius, sp. 52. 1 St.

1567...10 T. Alata. Der gellig gelte Thurm. 3 Sto 
1568...11 T. Rustica. Der rothe Thurn. Gn. Murex babylonus, sp. 52. 1 St.

1569...12 T. Pyranidalis. Der Pyramiden-Thurm. Gm. Murex babylonus, sp. 52. 2 St.

1570...13 T. Vitrea. Des gläserne Thurm. Gm. Murex babylonus, sp. $52.3 \mathrm{St}$.

1571...14 T. Regia. Der Königs-Thưm. Gm. Murex babylorius, sp. $52 \beta$. Mart. 4 p. 143 ving. 39 Lt. C. 3 Stück.

1572...14* T. Albida. Der weisse Thurm. Gm. Strombus lividus, sp. 49. 3 St.

$1575 . .14^{* *}$ T. Fossilis. Der ausgegrabene Thurin. Gm. Str. lividus, sp. 49. 1 St.

1574...14 *** T. St. Stephani. Der Stephans-Thur'm. Gmel. Strombus lividus, sp. 49. Chemn. 9 tab. 135 fig. 1269, 70. 1 St.

1575..15 T. Vetusta. Der alte Thurm. Gm. Strombus lividus, sp. 49. 1 St.

1576...16 T. Tornatum. Der gedrechseltc Thurm. Gm. Murex 'babylonus, sp. 52. Favanne tab. 33 fig. C. 6 . 4 Stück.

*1577..17! T. St. Mauritii, Der St. Moritz-Thurm. Gmel. Murex babylonus, sp. $52 \%$ Mart. 4 p. 143 ving. 39 fig. B. Argenv. zoomorpl., tab. 4 fig. 6 . Sehr sélten und schön. $2 \mathrm{St}$.

15\%8...18 T. Operosa. Der künstliche Thurm. Gm. Strombus vittatus, sp. $25 \gamma$. Favanne tab. 20 fig. A. 8 . Chemn. 10 tab. 155 fig. 1481 , 82. 2 St.

I579...19 T. Clathrata. Der gegitterte Thurm. Gm. Strom* bus vittatus, sp. 25 var. 1 St.

\section{Tritonium. Das See-Horn.}

1580...1 T. Tritonis. Das Tritons-Horn. Gm. Murex tritonis, sp. 89. Mart. 4. t. 134 fig:1272. 1282, 83. Knori Vergn: 2 tab. 16 fig. 2, $8 \& 5$ tab. 5 fig. 1.9 St. wovon 1 St. sehr gross.

$1531 \ldots-\alpha-1$ - -1 St.

1582... - $\beta$ T. - - das gelbe Tritons.Forn, Mart. 4 tab. 134 fig. I279. 1 Stiick. 
1583...2 T. Neptuni. Das Wasser-Horn. 4 St.

1584...3 T. Opis. Das verkürzte Tritons-Horn. Gm. Murex tritonis, sp. 89. $\beta$. Nart. 4 tab. 136 f. 1284, 85. 2 St. 1585... T T. Filiforme. Das kleine fein granulirte Tritons. Horn. 1 St.

1586...5 T. Minutum. Das hleine Tritons-Horn. 2 St.

1587...6 T. Buccinulum. Die kleine Trompete. Gm. Buccinum igneum̀, sp. 107. Mart. 4 t. 127 f. 121\% 1 St.

1588...7 T. Accinctum. Das gefieder te Tritons.Horn. Gmel. Buccinum plumatum, sp. 108. Mart. 4 tab. 127 fig. 1218, 19. 3 St.

1589...-T. \& - - Mart. 4 t. 127 f. 1220. 3 St.

1590...8 T. Maculatum. Das gefleckte Tritons-Horn. Gmel. Murex maculosus, sp. 79. Mart. 132 f. 1257, 58. $10 \mathrm{St}$.

*1591..9 T. Cundisatum. Das kandirte Tritons-Horn. Gmel. Murex conditus, sp. 174. Chemn. 10 t. 162 f, 1544, 45. Sehr selten und schön. $1 \mathrm{St}$.

1592...- - - Die Frosch-Schnecke. 1 St.

1593...10 T. Olearium. Der Oelkrug. Gm. Murex olearium,

sp. 27. Mart. 4 t. 130 f. 1243. Know Vergn. 9 t. 9 f. 5.1 St.

$1594 \ldots-\alpha-\alpha$ - 2 St.

1595... - $\beta$ - Mart. 4 t. 131 f. 1250. 1 St.

159i...11 T. Olfactoriolum. Die Riechbüchse. Gm. Murex olearium, sp. 2\%. 2 St.

$1597 \ldots-\alpha-\ldots$ - 1 St.

$1599 \ldots-\beta--2$ St.

1599...12 T. Lotorium. Das Waschbecken. Gm. Murex olearium, sp. 27. Mart. 4 .t. 130 f. $1248,49$.

1600...13 T. Labrum. Die Wanne. Gm. Murex olearium, sp. 27. 2 St.

1601...14 T. Nicobaricum. Das Nicobarische Tritons-Horn. Gnel. Murex olearium, sp. 27. Mart. 4 t. 132 fig. 1246, 47. 7 St.

1602...15 T. Limbatum. Das gesäumte Tritons-Horn. Gmel: Murex olearium, sp. 27. Mart. 3 t. 132 f. 1259. 1 St.

\section{Lade 66.}

1603...16 T. Hepaticum. Die gelbe Paternoster-Schnecke. Gm. Murex Rubecula, sp. 35. Mart. 4 t. 192 f. 1263, 61, 1 St. 126 
1604...17 T. Flaveola. Die dunkelbraune Paternoster-Schnecke. Gm. Murex Rubecula, sp. 35. Mart. 4 t. 132 f. 1261, 1262. $3 \mathrm{St}$,

1605...18 T. Rubecula. Die orangefarbene Paternoster-Schnecke. Gmel. Murex Rubecula, sp. 35,. Mart. 4 tab. 132 f. 1265,66 . $3 \mathrm{St}$.

$1605 \ldots$ - \% - 1 St.

1607...19. T. Natator. Der Schwimmer. Gm. Murex gyrinus, sp. 24. Mart. 4 t. 128 f. 1229, 30. 4 St.

1608...20 T. Gyrinus. Die kleine Kröte. Gm. Murex gyrinus, sp. 24. Mart. 4 t. 128 f. $1235,34.7$ St.

$16 c 9 . . .-\alpha-1$ St.

1610...21 T. Jabick. Die gefleckte Kröte. Gmel. Murex gyrinus, sp. 24. Mart. A t. 127 f. 1224 , 25. 6 St.

$1511 . . .-\alpha-1 \mathrm{st}$.

1612...22 T. Granulare. Das gehörnte Tritons-Horn. Gmel. Murex gyrinus, sp. 24. Mart. 4 tab. 127 f. 1226, 27. 4 Stück.

1613...23 T. Scrobiculator. Das geflammte Tritons-Horn. Gm. Murex scrobiculator, sp.36. Mart. 10. t. 163 f. 1556, 57. 2 St.

1614...24 T. Tuberosum. Das gebuckelte Tritons-Horn. Gm Murex Lampas rubeta, sp. 26. \% Mart. 4 t. 128 fig $1236,3 \% 1$ St.

*1615:.25 T. Argo Buccinum. Der gebandete Argus. Gmel. Murexargus, sp.78. Sehr selten. Mart.1223. Knorr 5 t. 3 f. 3. 7 St. wovon 2 ganz vorzüglich.

1616...26 T. Rubeta. Der Garten-Frosch. Gm. Murex Lampas, sp. 26. $\%$. Rubeta. 5 St.

1617... - $\propto$ - dünnschaligte. Mart. 4 t. 128 f.1235, 3\%. 2 Stück.

1618...27 T. Bufo. Die grosse Fiüte. Gm. Murcx Lampas, sp. 26. ß. "Bubo. Mart. 4 t. 129 f. 1238. 2 St.

1619...28 T. Hector. Der Hector. Gm. Murex lotorium, sp. 30. $\beta .1$ St.

1620..29 T. Simpulum. Gm. Murex lotorium. sp.30. $\beta$. Martini 4 t. 131 f. 1254.7 St.

1621...- - - - Gm. Murex lotorium, sp. 30. B. Mart. 4 t. 131 f. $1252,53.1 \mathrm{St}$.

$1622 . . .-\beta--3 S t$. 


\section{Lade 67.}

1623...30 T. Reticulans. Das gegitterte Tritons-Horn. Gmel. Murex reticulans, sp.37. Mart.4 t.128 f.1228. 6 St.

\section{Bursa. Die Tasche.}

1624... B B. Rana. Der Frosch. Gm. Murex rana, sp. 23. Mart. 4 t. 133 f. 1276. 6 St.

1625... - - - a Niart. 4 t. 133 f. 12\%0. 4 St.

1625... - $\beta$ - - a Mart. 4 t. 133 f. 1268, 69. 2 St.

1627...2 B. Gibbosa. Die bäuchigte Tasche. Gm. Murexrana,

sp. 23. a Mart. 4 t. 153 f. 1273. 2 St.

1628.:3 B. Mammata. Die wahre Kröte. Gm. Murex bufonius, sp. 32. Favanne Tb. 32 f. B. 1. Mart. 4 t. 129 f. $1240,41.2 \mathrm{St}$.

I629...3* B. Monitati. Die eingefasste Tasche. Gm. Murex bufonius, sp. 23. 1 St.

1630...4 B. Bufonia. Die stachlichte Kröte. Gmel. Murex rana, sp. 23 ß. Martini 4 t. 133 f. 1274, 75. 7 St. $1631 \ldots-\alpha-\infty$ St.

\section{Cymatium. Die Kehl-Leiste.}

1632...1 C. Femorale. Das dreyehige Kink-Horn. Gm. Murex femorale, sp. 28. Mart. 3 t. 111 f. 1039. Knorr Vergn. 4 t. 16 f. 1, 7 St.

$1633 \ldots-\alpha-2 S t$.

$1634 \ldots-\beta-1$ St.

1635...2 C. Fithinoceros. Die Hautschnecke. Gm. Murex py. rum, sp. 33- Knorr 6 t. 25 f. 2. 4 St.

\section{Lade 68}

1636...3 C. Pyrum. Die getrocknete Birne. Gmel. Murex pyrum, sp. 33. Mart. 3 t. 112 f. 1040-44. 4 St.

$1637 \ldots$... - 1 - St.

1638...4 C. Maculatum. Die-gefleckte Birne. 2 St.

1699...5 C. Clavatum. Die gegitterte Birne. Gmel. Murex pyrum, sp. 33. 1 St.

164̣0...6 Canalicularum. Die bräunliche Birne. Gm. Murex pyrum, sp. 33. 2 St. 
1641...7 C. Caudatum. Die weisse keilförmige Birne. Gmel. Murex pyrum, sp. 39. 2.St.

1642...8 C. Flexussum. Die gebogene Birne. Gm. Murex py. rum, sp. 33: 2 St.

$1643 \ldots-\alpha-1$ St.

1641...9 C. Minicatum. Die dornigte Birne. Gmel. Murex -pyrum, sp. 33. 1 St.

\section{Cabestana. Die Winde.}

1645...1 C. Cymiatium. Das knotigte Weinfass. Gm. Murex cutaceus, sp. 29. Mart. 3 t. 118 f. $1085,86.1$ St.

$1646 \ldots-\infty-1$ st.

$1547 \ldots-\beta-1$ - -1 st.

1648...2 C. Doliata. Das geripte Weinfass. Gmel. Murex curaceus, sp. 29. Die Hautšchnecke. Mart. $\mathcal{S}$ t. 118 f. $1087,88.8 \mathrm{St}$ :

$1649 . . .-\alpha-1 \mathrm{St}_{\text {. }}$

1650...3 C. Costata. Das dünschaligt geripte Weinfass. Gm. Murex cútaceus, sp. 29. Mart. 3. t. 118 f. 1087, 88. 2 Stück.

1651...4 C. Doliolum. Das Fässchen. Gnel. Murex decussatus, sp. 9. Mart. 3 t. 110 f. 1027. 7 St.

1652...5 C. Helciarum. Cabestan, oder die Schiffswinde. Gm Buccinum scala, sp. 61. Mart. 3 t. 118 f. 1089. Knorr Vergn. 3 t. 7 f. 2. $2 \mathrm{St}$.

1653... - - - - Schröters Einleit. in die Conch. 1 p. 360 t. 2 f. 8 . 2 St.

${ }^{*} 1654 . .-\beta-\beta$ - sehr selten. I St.

1655...6 Difformis. Die ungestaltene Winde, 1 St,

\section{Nucella. Die Nuss.}

1655...1 N. Reticulata. Dié gegitterte Nuss. Mart. 3 t. 121 f. $1107,8.3$ St.

1657...2 N. Moschatellina. Die Muscat-Nuss. Gm. Buccinun laeve, sp. 72. Mart. 4 t. 124 f. 1150 . s St. 1658...3 N. Macina. Die grosse Muskat-Nuss. 2 St. 1659...4 N. Lap:llus. Die steinigte Nuss. Gm. Buccinum rusticum, sp. 65. Mart. 3 t. 120 f. 1104 , 5. 6 St.

1660,..5 N. Theobroma. Die hakao-Nuss. Gm. Buccinum filosum, sp.67. Mart. 3 t.121 f.1113, 14. 1 St. 


\section{Lagena. Die Flasche.}

1661...1 L. Undosa. Die gewellte Flasche. Gm. Buccinum undosum, sp. 84. Mart. 4 t. 122 fo 1126, 2\%. 7 St.

1662...2 L. Crenulata. Die wulstige Flasche. Gm. Buccinum undosum, sp. 84. Mart. 1145, 46. 1 St.

1663...3 L. Fiolacea. Die bläuliche Flašche. 1 St. 1664...4 L. Elegantissima, Die orangefarbene Flasche. $2 \mathrm{St}$. $1665 \ldots$ - $\alpha-1 \mathrm{St}$.

1666...5 L. Varia. Die bunte Flasche. 1 St.

I667...6 Nodulosa. Die knotige Flasche. 1 St.

1668...7 Pterygiata. Die geflïgelte Flasche. 2 St:

1669...8 L. Nigella. Die schäkigte Flasche. Gm. Buccinum ocellatum, sp. 73. Mart. \& t. 124 f. 1160, 61. I St.

\section{Nassa. Die Fisch-Reuse.}

1670... I N. Picta. Die gemahlte Fischreuse. Gm. Buccinum coronatum, sp. 68. Mart. 3 t. 121 f. $1115,16.5$ St.

1671...2 N. Caesia. Die braune Fischreuse. $1 \mathrm{St}$.

1672...3 N. Flaveola. Die lichtbraune Fischreuse. I St.

1673...4 N. Striata. Die gestreifte Fischreuse. 1 St.

1674...5 N. Duplicata. Die weissgelbe Fischreuse. I St.

1675...6 N. Legata. Die gebandete Fischreuse. Gm. Buccinum filosum, sp. 67: Mart, Tom. 3 tab. 121 fig. $1113,14.1 \mathrm{St}$,

1676...7 N. Rudis, Die plumpe Fischreuse. Gm. Buccinum lapillus, sp. 53. Mart. 3 t. 121 f. 1111, 12. 4 St.

$1677 \ldots 8$ N. Harpa. Die Harfe. 1 St.

1678...9 N. Argiolus, Die äugichte Fischreuse. $1 \mathrm{St}$.

\section{Cantharus. Die Kanne.}

1679...1 C. Globularis. Die kugelförmige Kanne. Gm. Buc. cinum tranquebaricum, sp. 86. I St.

1680...2 C. Tuberosus. Die knolligte Kanne. Gm. Buccinum undosum, sp. 84. -Mart. 1146, 4\%, 6. St.

1681...3 C. Scalaris. Die gewundene Kanne. 2 St.,

1682...4 C. Nodosus. Die knotige Kanne, 1 St.

1683...5 C. Erosus. Die blättrige Kanne. Gm. Murex hipe 
$1684 \ldots 5$ * C. Hippocastanum. Die wilde Castanie. Gm. Murex hippocastanum, sp. 48. 6 St.

$1685 \ldots$... - Nart. 3 t. 100 f. 951. 4 St. monströs.

*1686.. 5 ** C. Tribuloides. Die gedornte Kanne. 1 St. selir schün.

1687...6 C. Triplicatus. Die gefaltete Thanne, Gm. Buccinum pyrozonias, sp. 71. Mart. 3 t. 109 f. 101\%. 4 St.

\section{Distorsio, Das Ohr-Horn.}

1. Caudatce. Geschwänzte.

1683...1 D. Anus. Das Ohrhorn. Gm. Murex anus, sp. 38. Mart. 2 tab. 41 fig. 403, 4. Knorr 3 tab. 3 fig. 5 . 5 Stïck.

1689...2 D. Reticulata. Das gelbe Ohrhorn. Gmel. Murex anus, sp. 38 var. Mart. 2 t. 41 f. 405, 6. 5 St.

1690...3 D. Clatrata: Das weisse Ohrhorn. Gmel. Múrex anus, sp. 38 var. 1 St.

1691...4 D. Muricina. Das stachlichte Ohrhorn. Gm. Mu• rex pyrum, sp. $33 \delta$. Mart. 3 tab. 112 fig. 1050, 51. 4. Stück.

\section{Truncatce. Abgestutzte.}

1692...5 D. Arcularia. Das kofferartige Ohr. Gm. Buccinum arcularia, sp. 42. Mart. 2 t. 41 f. 409 , 10. 6 St.

1693...6 1). Plicata. Das gefaltete Ohrhorn. Gm. Buccinum arcularia, sp. 42. Mart. 2 t. 41 f. $411,12.4$ St.

1694...7 D. Dealbata. Das gelbliche Ohrhorn. Gm. Buccin. arcularia, sp. 42 var. 3 St.

*1695..7* ! D. Subalata. Das geflügelte Ohrhorn. Gmel. Buccinum arcularia, sp. 42 var. 1 St.

1695...8 D: Communis. Das dunkelbraune Ohrhorn. Gmel. Buccinum arcularia, sp. 42 var. Mart. 4 tab. 124 fig. 1169. 4 St

\section{Lade 69.}

\section{Xancus. Das Sianco-Horn.}

1697...1 X. Pyrum. Die Bime. Salbliorn. Gm. Voluta pyrum, sp. 102. Mart. \& tab. 95 fig. 916. Knorr 6 tab. 29 fig. 1. $2 \mathrm{St}$. 
$1698 \ldots-\infty-1$ - -1 t.

$1699 \ldots-\beta-1$ St.

$1700 \ldots-\gamma-2$ St.

1701...2 X. Purictatus. Die bunte Birne. Gm. Voluta pyrum, sp. 102. 2 St.

1702...- - - - Mart. 3 tab. 95 fig. 918. Knorr 6 tab. 27 fig. 2. 3 St.

*1703..3 !! X. Perversus. Das linke Sianko-Horn, äusserst scl. ten. Chemn. 9 tab. 104 fig. 884, $85 \%$. 1 St.

\section{Pyrene. Der Judenstein,}

1794...1 P. Rhombiferum. Der gefleckte Judenstein. Mart, 2 tab. 44 fig. 465. Der goldgelbe weisse Olivenkern. 1 Stück.

\section{Terebellum. Der Bohrer.}

1705...1 T. Nebulosum. Der gebardete Bohrer.' Gm. Bulla terebellum, sp. 22. Mart. 2 t. 51 fig. 568, 69. Lister tab. 736 fig. 30.6 St.

1706...2 T. Lineatum. Der liniirte Bohrer. Gm. Bulla tere. bellum, sp. 22 3. Iister t. 735 f. 31. 3 St. 1707... T T. Punctulatum. Der punctirte Bolner. Lister tab. 737 fig: 32. 1 St.

\section{Mitra. Die Mütze.}

1. Anfractibus coronatis. Mit gekrönter Windung. 1708...1 M. Papalis. Die Pabst-Krone. Gn. Voluta papalis, sp. 95. Mart. 4 t. 147 f. $1353,54.9$ St.

$1709 \ldots-\infty$ - - s St.

1710...2 M. Gardinalis. Der Kardinals-Huth. Gmel. Voluta papalis, sp. 95 3. Mart. 4 t. 147 f. 1355, 56. 9 St. 1711...3 M. Imperialis. Die Kayserkrone. Gm. Voluta pertusa, sp. $92 \%$ Chemn. 10 t. 151 f. 1432,33 . S St. 1712...4 M. Aurata. Die goldne Krone. Gm. Voluta polygona, sp. 62. Mart. 4 t. 150 f. $1401,2.1$ St. $1713, \ldots 5$ M. Bivertex. Die zweiwirblichte Pabstkrone. 1 St。 1714...6 M. Granularis. Die besetzte Pabstkrone. 1 St. 1715...7 M. Stricta. Die gestrichelte Pabsthrone, $2 \mathrm{St}_{2}$ 
1716...8 M. Bernhardina. Die Bernhardiner Pabstkrone. Gm. Volutá patriarchalis, sp. 198. Chemil. 10 tab. 150 fig. 1427. 2 St.

*1717..8* M. Perlata. Die geperlite Pabsthrone. Gm. Voluta, pertusa, sp. 83 var. selten. 2 St.

2. Anfractibus contiguis. Mit aneinander stehender Windung.

1718...9 N. Episcopalis. Die Bischofsmütze. Gmel. Voluta episcopalis, sp. 94. Mart. 4 tab. 147 f. 1360, 1360 a. 9 Stück.

$1719 . . .-\infty-5$ St.

1720...10 M. Carmelita. Die Carmelitermǔtze. Gm, Voluta episcopalis, sp. 94. $2 \mathrm{St}$.

$1721 . . .-\alpha-2$ St.

1722...11 M. Monachialis. Der Mönchs-Huth. Gm. Voluta cardinalis, sp. 93. Mart. 4 t. 147 f. 1358 59. 5 St.

1723...12 I. Pertusa. Die durchstochene Pabstkrone. Gmel. Voluta pertusa, sp. 83. Kammerer 9 f. 8 I St.

\section{Lade 70.}

1724...13 Mr. Eremiitarum. Der Einsiedler-Huth. Gm. Voluta pertusa, sp. 93. Mart. 4 t. 147 fo 1361. 3 St.

1725...14 M. Capucina. Die Capuzinermütze. Gm. Voluta pertusa, sp. 93 var. 1 St:

$1726 \ldots-\infty-1 \mathrm{St}$.

172\%...15 M. Lutea. Die gelbe Bischofsmütze. $2 \mathrm{St}$.

1728...16 Die weissgesprenkelte Bischofsmütze. $2 \mathrm{St}$.

1729..17 M. Discolor. Die weissgebandete Bischofsmütze, Gm. Voluta scutulata, sp. 131. Chemn. 10 tab. 151 fig. $1428,29.5$ St.

1730...18 M. Castanea. Dic castanienbraune Bischofsmütze. Gm. Volúta nigra, sp. 182. Chemn. 10 tab. 151 fig: 1430, 31. 1 St.

1731...19 M. Vittata. Die buntschächige Bischofsmütze. 1 Stück.

1732...20 M. Venosa. Dic adrigte Bischofshütze. Gm. Vo• luta paupercula, sp: 37: Mart. \& tab. 149 fig. 1386, 87. 3 St. 
1793...21 M. Nivea. Die schneeweisse Bischofsmütze. 1 St. 1734...22. M. Minuta. Die kleine Bischofsmütze. Gm. Voluta aurantia, sp. 60. Märt. 4 tab. 150 fig. 1393, 94. 1 Stück.

*1735..23 M. Crenulata. Die ausgezackte Bischofsmütze. Gm. Voluta crenulata, sp. 130. Chemn, 10 tab. 150 .fig. 1413, 14. Selten. 1. St.

3. Anfractibus distinetis. Mit von einander abgesonderter Windung.

1736...24 M. Elata. Die feingegitterte Bischofșmütze. Gm. Voluta acuminata, sp. 63. Mart. 4 tab. 150. fig. 1403, 4. 1 St.

1737...25 M. Cinnamomea. Die grobgegitterte Bischofsmütże. 3 Stück.

1738...26 M. Elegantissima. Die niedliche Bischofsmütze. 1 Stiick.

1739...27 M. Pulcherrima. Die schöne Bischofsmütze. 3.St.

1740..28 M. Praestantissima. Die überaus schöne Bischofsmütze. Gm. Voluta scabricula; sp. 48. Mart. 4 tab. 149 fig. 1388, 89. 4 St.

1741...29 M. Flammeola.' Die geflammte Bischofsmütze. 1 St. 1742...30 M. Sanguisigna. Die mit Blut gezeichnete Bischofsmütze. Gm. Voluta sanguisaga, sp. 50. Mart: 4 tab. 148 fig. 1373. 5 St.

$1743 . . .-\alpha-2 \mathrm{St}$ -

1744...81 Mr. Sanguinea: Die blutrothe Bischofsmütze. 1 St. 1745...32 M. Cancellata. Die gewürfelțe Bischofsmütze. Gm. Voluta granosa, sp. 136. Chemn. 10 tab. 151 fig. 1442, 43. 1 St.

1746...33 M. Caelata. Die gestreifte Bischofsmütze. 1 St. 17.47...34 M. Nobilis. Die Orange Bischofsmïtze. 1 St. $1748 \ldots 35 \mathrm{M}$. MTargaritacea. Die geperlte Bischofsmütze. 1 St,

\section{Vexillum. Die Fahne.}

1749...1 V. Fossile. Die weisse Flagge. $1 \mathrm{St}_{0}$ 1750...2 V. Plicatum. Die gefaltete Flagge. Gm. Voluta plicaria, sp. 55. Mart. 4 t. 148 f. $1362,63.4$ St.

1751...3 V. Lividum. Die bleifarbige Flagge. Gmel. Voluta plicaria, sp. 55. $6 \mathrm{St}_{\mathrm{s}}$ 
1752...4 V. Variegatum. Die veränderliche Flagge. Gmel. Voluta variegata, sp. 89. Knorr. 5 t. 18 f. 6.

$1753 . . .-\infty-3 \mathrm{St}_{\text {. }}$.

$1754 \ldots-\beta-\beta$ - 5 St.

$1755 \ldots-\gamma-\gamma$ St.

$1756 \ldots-\delta-1 S S_{0}$

$1757 \ldots-\varepsilon-1$ - 4 St.

$1758 \ldots-$ - -7 St.

1759...5 V. Rufun. Die rothe Flagge. Gmel. Voluta varie. gata, sp. 89. 2 St.

1760...6 V. Subdivisum. Die weissliniirte Flagge. Gm. Voluta subdivisa, sp. 139. Chemn. 10 tab. 151 f. 1436, 3\%. 5 St.

$1761 . .7$ V. Caffrum. Die gelbgebandete Flagge. Gm. Voluta caffra, sp. 51. Mart. 4 t. 148 f. 1369, j0. 6 St.

1762...8 V. Superbum. Die Admirals-Flagge. Gmel. Voluta plicaria, sp. 55. Mart. 4 tab. 148 f. 1365. 1 St.

*1763..9 V. Gloriosum. Die orange Flagge. $1 \mathrm{St}$. 1764...10 V. Laetum. Die Freuden-Flagge. 2 St. .

\section{Lade 71.}

\section{Purpura. Die Purpur-Schnecke.}

. 1. Poligonoe. Vieleckigte.

a. Tuberculatae. Knoblichte.

*1765..1 P. Onagrina. Das Chagrin-Horn. Gm. Murex miliaris; sp. 39, äusserst rar. d'Argenv, tab. 4 f. 1102. Mart. 3 p. 303 vign. 36 fig. 1, 5 . 4 St.

$1766 \ldots$ - $-\infty$ - $2 \mathrm{St}$.

1767...- $\beta$ - - Chemn. 10 tab. 161 frg. 1532-35. I St.

1768...2 P. Trunculus. Der braungezackte Hochschwanz.. Gm.

Murex trunculus, sp. 5. Mart. 3 tab. 109 fig. 1018-

20. 13 St. 1 St. bewachsen mit Seetulpen.

1769...- - - - St.

$1770 \ldots-\beta-1$ St.

$1771 \ldots-\gamma-15 \mathrm{St}$.

1772...3 P. Tribulus. Die Wasser-Nuss, Gm. Murex truncu• $\mathrm{luss}_{2} \mathrm{sp}, 5,5 \mathrm{St}$. 
B. Crispatae. Gehräuselre.

${ }^{*} 17 \% 3 . .4$ !! P. Buccinulum." Die ächte Bords-Treppe. Schr selten. $1 \mathrm{St}$.

1774...5 P. Lancellata. Die Bords.Treppe, 2te Sorte. 1 St. 1775...6 F. Senegalla. Die Senegalsche Purpur-Schnecke. 1775...7 P. Costata. Die geribte Purpur-Schnecke. Gm. Miurex costatus, sp. 86. 5 . St.

1777...8 P. Congener. Die weissgelbe Purpur-Schnecke. Gm. Murex decusatus, sp. 7. Mart. 3 tab. 110 fig. 1026. Knorr 4 tab. $23 \mathrm{fig}$. 3. $1 \mathrm{St}$.

1778...9 P. Crispata. Die hiause Purpui-Schneche. 9 St. 1779...10 P. Acuta. Die scharfe Purpur-Schnecke. 1 St. 1730...11 P. Rubicunda. Die röthliche Purpur-Schnecke. Gm. Murex trunculus, sp. 5.3 St.

1781...12 P. Rosarium. Die rosenrothe Purpur. Schnecke. Gm. Murex trunculus, sp. 5 \}. Chemn. 10 tab. 161 fig. 1528, 29. $3 \mathrm{St}$.

1782... - $-\infty-1$ St. mit scharfen Stacheln.

1783...13 P. Virginea. Das Jungfern-Horn. Gm. Murex ramosus, sp. 13 \&. Mart. 3 t. 110 f. 1030. 7 St. $1784 . . .-\infty-$ Mart. 3 t. 110 f. 1029. 3 St.

\section{Lade $\tau 2$.}

1785...11 P. Duplex. Die Purpur-Schnecke mit blutrother Mündung. Gm. Murex saxatilis, sp. 15 ß. Mart. 3 t. 108 f. 1013, 14. 1 St.

\% Fimbriato frondosae. Mit Laub bewachten.

1786...15 P. Lactuca. Der Kopfsalat. Gm. Murex saxatilis, sp. 15. Mart. 3 t. 107 f. $1005-8$. 3 St $_{\text {s }}^{\text {(1'Stück }}$ ganz vorzüglich.

178\%...- u - - Mart. 3 t. 107 f. 1011, 12. 4 St.

1788...16 P. Scorpio. Der Scorpion. Gm. Murex scorpio, sp. 14. Mart. 3 t. 106 f. 998-1003. Knorr 2 tab. 11 fig. 4, 5. 2 St.

1789...17 P. Elongata, Die ausgestreckte Purpur-Schnecke. 1 Stück.

1790...18 P. Tuberósa. Die mit schwarzen Strallen besetzte Purpur-Schnecke. Gm. Murex melmamathos, sp.9. Mart. 3 t. 108 f. 1015. $3 \mathrm{St}$. 
*1791...18*!! P. Martinetana. Die gefensterte Purpur'schnecke. Gm. Murex colus, sp. 61. ठ. Chemn. 10 tab. 161 fig. $1536,37.1$ St.

141 1792...19 P. Spinula: Die gedornte Purpur-Sclinecke. 1 St. 1793...- $\alpha \div-$ Tour d'Auvergne $11288^{* *}$. $1 \mathrm{St}_{j}$

2. 'Trigono. Dreieckige.

๙. Cuspidatae. Zugespitzte.

1794.. .20 P. Cornu Cervi. Das Hirschhorn. Gm. Murex ramoșus, sp. 18. Mart. 3 tab. 102 fig. 987, 88. 1 St. Ein Exemplar selir gross.

1795...21 P. Corku Damae, Das Damm-Hirschhorn. eod: 6 Stück:

\section{B. Frondosáe. Aestige.}

1796...22 P. Brinnea. Die braune Purpur-Schnecke. eod, 6 Stiuck. $1797 \ldots-\alpha-4$ - 1.

1798...22* P. Quadricornis. Die vierhornigte Purpurschnecke. eod. 2 St.

1799...23 P. Ramosa. Die liniirte Puipur-Schnecke. eodem. 7 Stück.

1800...- $\propto$ - - Mart. 3 tab. 102 f. 981, 82. 2 St.

1801...24 P. Gemella. Die lichtbraune Purpur-Schnecke. 4 Stück.

\section{Lade. 73.}

1802..25 P. Lactea. Die milchweisse Purpur-Schnecke. 4 St. 1803..26 P. Uncinata. Die mii Haken besetzte Purpur-Schn. 1804...27 P. Incarnata. Die röthliche Purpur-Schnecke. Mart.

3 t. 109 fig. 980, 81. 10 St. wovon 1 St. sehr gross. 1805..28. P. Carneola. Der Rosenstock. eod. Mart. 3 tab. 102. fig. 995, 96. 3 St.

1806...29 P. Brandaris. Das Brandhorn. Gmel. Murex brandaris, sp.4.- Knorr Verg. 2. t. 18 f. 1, 2 \& 22 f. $4,5$. $12 \mathrm{St}$.

$1807 \ldots-\infty \cdot-11$ St.

1808..30 P. Fuliginosa. Die russfarbene Purpur-Schnecke. Gmel. Murex brandaris, sp. 4 \% $8 \mathrm{St}$.

$1809 . .0-\alpha-7 \mathrm{St}_{\mathrm{t}}$ 


\section{Lade 74.}

$\%$ Optusae. Abgestumpfte.

1810...31 P. Capucina. Die Kapuziner Purpur-Schnecke. Kettenhorn (mit stumpfen Näthen) Gm. Murex ramosus, sp. 13. Tour d'Auverg. 1073. \%. Mart. 3 t. 105 f. 994.4 St.

1811...32 P. Variegata. Die schàckigte Purpurschnecke.. Gm. Murex ramosus, sp.13. ל. Mart. 3 t. 111 f. 1038. 3 St. $1812 . . .-\alpha-2$ St.

1813...33 P. Cancellata. Die gegitterte Purpur-Schnecke. Gm.

Murex ramosus, sp. 13. ל. Mart. 3 t. 111 f. 1038. 2 St.

1814...3S * P. Despecta. Die zalnneiche Purpur-Schnecke. ISt, 1815...34 P. Truncata. Die abgestumpfte Purpur-Schnecke. Gmel. Murex decussatus; sp. 7. Chemn. 10 t. 161 f. 1540,41 . $2 \mathrm{St}$.

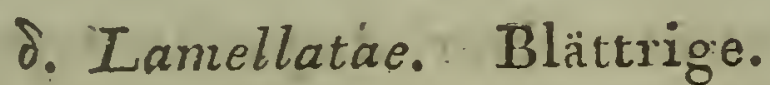

*1816.35 ! P. Foliata. Die blättrige Purpur-Schrecke. Gm. Murex foliarus, sp.'174; selten: Chemn. 10 t. 161 f. 1538, 39. Mart. univ. Conch. 2t. 66. 2St. Sehr schön.

1817...36 P. Alata. Die weisse géflügelte Purpur-Schnecke, Gm. Murex ramosus, sp.13; \&. Mart.3 t. 111 f. 1036 . 37. 1 St.

1818...37 P. Crenulata. Die gekerbte Purpur-Schnecke. Gm. Murex asper, sp. 60: Brander Fossilia Hantoniensia, p. 3.f. $77,79.1$ St.

1819...38 P. Fusiformis. Die gespindelte Purpur-Schnecke. $\mathrm{Gm}$. Murex ramosus, sp. 13. $3 \mathrm{St}_{\text {. }}$

1820...39 P. Draco. Dei Drache. Gm. Murex tuba, sp. 103. Mart. 8 t. 111 f, 1033: 2 St.

\section{Murex. Die Stachel-Schnecke.}

1821...1 M. Haustellum. Der Schnepfenkopf. Gmel. Murex haustellum, sp. 1. Mart. \& t. 115 f. 1066. 10 St. $1822 . . .-\alpha-2 \mathrm{St}$. *1823..1 * M. Purpuroides. Der stachlige Schnepfenkopf. Gm Murex haustellum, sp. 1 var. sehr selten. 1 St. 
02

1824... * M. Scolapaceus. Der keilförmige Schnepfen-Knopf. Favanne Tb. 38 fig. B. 2. 2 St.

1825...2 M. Cornutus. Die gezackte Herkules-Keule. Gmel. Murex cornutus, sp. 3. Mart. 3 t. 114 f. 105\% 21 St. 1826...3 M. Clava Herculis. Die Herkules-heule. Gm. Murex brandaris, sp. 4. 1 St.

1827...4 Tuberculatus. Der weisse Schnepfenkopf. Gm. Murex brandaris, sp. 4. Mart. 3 t. 114 f. 105s. 2 St,

\section{Lade 75.}

1828..5 M. Trapa. Der einfache Spinnenkopf. Gm. Murex tribulus, sp. 2. $\alpha$. Mart. 3 t. 113 f. 1055, 55. 3 St.j 1829...6.M. Tribulus. Der Fussangel, Gm. Murex tribulus, sp. 2. B. Mart. 3 t. 113 f. 1051, 52-54. 10 St.

*1850..7 M. Forskoehlii. Dèr forskoehlische Spinnenkopf. Gm. Murex tribulus, sp. 2. Der seltene gebandete Arabi. sche. 2 St.

*1831..8!! M. Histrix. Der doppelte Spinnenkopf. Gnel. Murex tribulus, sp. 2. Favanne t. 38 fig. A 1 \& 2. Knorr 5 to 26 f. 1.12 St.

\section{Tudicla. Die Irüchen-Kelle.}

1852... T. Carinata. Der gekehlte Schöpfer. Gm. Murex spirillus, sp. 64. Mart. 3 t. 115 f. 1069. Knorr 6 t. 24 f. 3. $3 \mathrm{St}_{\mathrm{s}}$

1833...2 T. Gutturnium. Der Schöpfer. Gm. Murex pyrum, sp. 33 ß. Mart. 3 t. 112 f. 1048, 49. 2 St.

\section{Pila. Der Ball.}

1834... I P- Manetou. Gm. Helix ampullacea, sp. $43 \beta$. Chemn. 9 t. 128 f. 1136 . I St.

1855...2 P. Equestris, Der gebandete Ball. Gm, Helix ampullacea, sp. 43. 2 St.

1836...3 P. Periscelis. Der weisse Ball. Gm. Helixampullacea, sp. $43 \delta$. d'Argenv. t. 17 fig. B. $2 \mathrm{St}$

1837...4 P. Anıpulacea. Das blaue. Band. Gm. Helix ampullacea, sp. 43. Chemn. 9 t. 128 f. 1133-35. 7 St。 
1838...5 P. Sepulta. Der ausgegrabene Ball von Courtaignon. Gm. Helix ampullacea, sp. 43. $3 \mathrm{St}$.

I839...6 P. Ignota. Dex schwarze Ball. Gm. Helix ampullacea, sp. 43. 1 St.

\section{Lade 76.}

\section{Cochlis. Die halbe Kugel.}

1840...1 C. Albula. Die weisse halbe Kugel. Gmel. Nerita spadicea, sp. 8. Chemn. 5 tab. 188 fig. 1896 a.b. 2 St, 1841...2 C. Explanata. Die abgebleichte halbe Kugel. Gm. Nerita orientalis, sp.12. Chemn. 5 t. 188 f. 1904 . 1 St. 1842...3 C. Cornea. Die hornfarbige halbe Kngel: Gm. Nerita spadicea, sp. 8. 1 St.

1843...4 C. Canrena. Der Schmetterling. Gm. Nerita cans tena, sp. 1. Mart. 5 t. 186 f. 1860, 61. 11 St. $1844 \ldots$ - C. $\alpha-1$ St.

1845...5 Ala Papilionis. Der Schmetterlings-Flügel. Gmel Nerita canrena, sp. $1 \delta$. Chemn. 5 t. 186 f. 1868-71, Knor: 1 t. 10 f. $5.1 \mathrm{St}$.

1845...6 C. Clausa. Die ungenabelte halbe Kugel. 2 St.

1847...7 C. Flammea. Die flammigte halbe Kugel. Gm. Nerita vittata, sp. 18. Chemn. 5 t. 188 f. 1917. 2 St. 1848...8 C. Fanel. Die buntschächigte halbe Kugel. Gmel. Nerita canrena, sp 1 \&. Chemn. 5 t. 187 f. 1875, 77" 7 St.

1849...9 C. Stercus Muscarum. Die gestippelte halbe Kugel. Gm. Nerita cantena, sp. 1 \}. Chemn. 5 t. 187 f. 1878-30. Nach der Beschreibung, aber nichtnach der Figur. 1 St. 1850...10 C. Ziczac. Die ziczac halbe Kugel. Gmel. Nerita fulminea, sp. 10. Chemn. 5 t. 187 f. 1881. 3 St.

$1851 \ldots-\alpha-2 \mathrm{St}$.

1852..11 C. Undulata. Die wellenfürmigc halbeshugel. Gm. Nerita canrena, sp. 1. Chemin. 5 t. 187 f. $1885,86$. 1 St.

1853...12 C. Milleporia. Die punctirte halbe Kugel. Gmel. Nerita canrena, sp. 1 ß. Chemn. 5 t. 186 f. 1862, 63. $2 \mathrm{St}$.

1854...13 C. Tigrina, Der Tíeger. Gm. Nerita canrena, sp.1, Chemn. 5 t. 187 f. 1892; 93. 4 St.

1855.,.14 C. Onca. Die Unke. Gm. Nerita canrena, sp. Io Chemn, 5 t. 187 f. 1887,88 . 1 St. 
1855...15 C. Pavinentum. Die wiurfliche halbe Kugel. Gm. Nerita canrena, sp. 1. Chemn. 5 t. 187 f. 1889, so. 2 St.

1857...16 C. Lineata. Die lineirte halbe Kugel. Gm. Nerita canrena, sp. $1 \%$ Cliemn. 5 t. 186 f. 1864, 65. 4 St. 1858...17 ! C. Plicata. Die gefaltete halbe Kugel. Gmel. Nerita rugosa, sp. 14. Chemu. 5 t. 188 f. 1902, 3. 1 St.

1859...18.C. Sulcata. Die gefurchte halbe Kugel. Gm. Nerita cancellata, sp. 2. Chemn. 5 t. 188 f. 1911, 12. 1. St. 1860...19 C. Vitellus. Der Eydotter. Gm. Nerita vitellứs, sp. 4. Chemn. 5 t. 186 f. 1866, 67. 2 St.

1861...20 C. Rufescens. Die braune weissgebandete halbe Kugel. Gm. Nerita spadicea, sp. 8. Chemin. 5 t. 19̧ f. $1872,73.2$ St.

\section{Ficus. Die Feige.}

1862...1 F. Communis. Die gemeine Feige. Gm. Bulla ficus; sp. 14. Knorr Verg. 3 t. 23 f. 1. 9 St.

1863...2 F. Variegata. Die bunte Feige. Gm. Bulla ficus, sp. 14. Mart. 3 t. 65 f. 734, 35. 7 St.

1854...3 F. Picta. Die schächigte Feige, eod. 4 St.

\section{Lade 7.7. Rapa. Die Rübe.}

1865...1 ! R. Globosa. Die weisse Rübe, dickschaligt, mit vertiefter Spitze. Gm. Bulla rapa, sp. 15. 2 St.

$1866 \ldots 1$ * R. Raphanus. Der Pettig. Gm. Bulla rapa, sp. 15. 1 Stück.

1857...2 P. Striata. Die Radies. Gm. Bulla rapa, sp. 15. Mart. 3 t. 68 f. $747-49$. 2. St.

1865...3 R. Pellucida. Die schneeweisse Radies. Gm. Bulla rapa, sp. 15. Mart. 3 t. 68 f. 748. 1. St.

we $1869 \ldots-\alpha-\ldots 2$ St.

1870...4 R. Volema. Die grosse gezachte Rïbe., Gm. Murex rapa, sp. 68. Mart. 3 t. 68 f. 750-53. Kuorr Verg. 5 .

t. 21 f. 2.1 St.

$1871 \ldots-\alpha-1$ - St.

$1872 . . \beta-\beta-$ - 4 St.

$1873 \ldots-\gamma-\gamma$ - 1 S t.

$1874 \ldots-\delta-1$. St.

$1875 \ldots-\varepsilon-1 \mathrm{St}$ 


\section{Busycon.}

1876...1 B. Muricatum. Die stachlige Feige. Gmel. Murex carica, sp. 67. Mart. 3 t. 67 f. 744 \& t. 69 f. 756.5 St. 1877...2 B. Perversum. Die linksgewundene Feige. Mart. 9.

t. 106 f. 902, s. 3 St.

1878... - \& - Mart. 9 t. 106 fig. 900, 1. 1 St.

1879... 3 B. Dubium. Die grosse linksgewundene Feige. 1 St. 1880...4 B. Cingulatum. Die gegürtelte Feige. $2 \mathrm{St}$.:

1881...5 B. Inversum. Die gegürtelte linksgewundene Feige. 1 Stück.

1882...6 B. Canaliculatum. Die gekrönte Feige. Gm. Murex canaliculatus, sp. 65 . Mart. 3 t. 67 f. 742, 49. 5 St.

\section{Lade 78.}

\section{Harpa. Die Harfe.}

1883...1 H. Major. Die grosse Harfe. Gm. Buccinum harpas sp. 47. Mart. 3 t. 119 f. 1090. Knorr Verg. 1 t. 9 f. $g$ \& 2 t. 8 f. $215 \mathrm{St}_{\text {。 }}$ $1884 . . .-\alpha-1$ St.

1855...2 H. Cythara. Dic Zitter。 Gm. Buccinum harpa, sp.47 $18 \mathrm{St}$.

1886...3 H. Nobilis. Die edle Haxfe. Gm. Buccinum harpa, sp. 47. Mart. 3 t. 119 f. 1091.1 St.

1887...4 H. Doris. Die rothe Harfe, eod. Mart. 3 t. 119 f. 1094。 3 Stïck.

1888...5 H. Amouretta. Die Harfe mit schmalen und breiten Pippen, eod. Mart. 3 t. 119 f. 109\% 7 St.

1889...6 H. Davidis. Die Davids-Harfe, eod. Mart. \& t. 119 f. 1092. 5 St.

1890 ..7 H. Cancellata. Die schmalrippige Harfe, eod. \& Chemn。 10 t. 152 f. $1453.7 \mathrm{St}_{\text {。 }}$

\section{Lade 79. \\ Cadus. Das Weinfass.}

1891...1 C. Perdrix. Das Rebhuhn. Gm. Buccinum perc̉ix, sp. 3. Mart. 3 t. 117 f. 1079. $5 \mathrm{St}$

1892.. - $-\alpha-$ - monströs. 1 St.

1893...2 C. Meleagris. Die gebandete Tonne mit Puncten, Gm. Buccinum perdrix, sp. 8. I st. 
1894...3 C. Coturnix. Die Wachtel. Gm. Buccinum perdrix, sp. 3. 6 St.

1895...4 C. Cepa. Die gewässerte Tonne. Gm. Buccinura olearium, sp. 1. Mart. 3 t. 117 f. 1076-1080. \& St.

1896...5 C. Cassis. Das Stüchfass. Gm. Buccinum dolium, sp. 5. 3 St.

1897...6 Galea. Die Biertonne, eod. 4 St.

1898...7 C. Globosus. Die Kugeltonne, eod. I St.

1899...8 C. Dolium. Die bunte Tonne, eod. Mart. \& t. 116

f. $1073,74.10 \mathrm{St}$.

1900...9 C. Doliolum. Das bunte Tönnchen, eod. 4 St.

1901...10 C. Albus. Die weisse Tonne, eod. 1 St.

1902...11 C. Diaphanus. Die durchsichtige Tonne mit zwei Mündungs-Säumen, eod. I St.

\section{Lade 80.}

1903...12 C. Fasciatus. Die braun gebandete Tonne. Mart.3 t. 117 f. 1081.4 St.

1904...13 C. Pomum. Die eyförmige Tonne. Gm. Buccinum pomum, sp.4. Mart. 2 t. 36 f. 370, 71. Knori Verg. 6 t. 23 f. 2. $4 \mathrm{St}$.

\section{Cymbium. \\ Der Thahn, oder die Tepel-Bake.}

1. Spira coronata. Die gekrönte Tepel-Bake. 1905...1 C. Aethiopicum. Der Mohren-Kiahn. Gmel. Voluta aethiopica, sp. 113. 8 St.

1906...- $\alpha-$ - Mart. 3 t. 75 f. 784. 1 St.

$1907 \ldots-\beta-1$ St.

1908...2 C. Persicum. Der persische Fiahn. Nart. $\mathbf{3}$ t. 75 f. 785. s St.

1909...3 C. Flammeum. Die bunte Tepel-Bake. Mart. 3 t. 74 f. 780.4 St.

1910...3* C. Senticosum. Die dónigte Tepel.Bake. Mart. 3 t. 76 f. 788.2 St.

\section{Lade 81.}

1911...4 C. Citrinum. Die citronengelbe Tepel-Bake, eod. $1 \mathrm{St} \cdot$ 1912...5 C. Marmoratum. Die marmorixte Tepel-Bake, eod. 1 St. 
2. Spira tecti. Mit bedeckter Tepel.

1913...6 C. Melo. Die Melone. Gm. Voluta olla, sp. 115. Mart. 3 t. 71 f. $766,67.1$ St.

$1914 \ldots-\alpha-2 \mathrm{St}$.

1915...7 C. Maculatum. Der gefleckte Kahn. Gmel. Voluta indica, sp. 120 . Mart. 3 t. 72 f. $772,73.5$ St.

$1916 \ldots-\alpha-2 \mathrm{St}_{\text {. }}$

3. Spira rotundata. Mit gerundeter Tepel. 1917...8 C. Philipinum. Der Philipinische Kahn. Gm. Voluta olla, sp. 115. Mart. 5 t, 71 f. 766.2 St.

$1918 \ldots 8$ * C. Inflatum. Die aufgeblasene Tepel-Bake. Gmel. Voluta navicula, sp. 118. Mart. 3 t. 71 f. 771. 2 St. $1919 \ldots 9$ C. Guttatum. Die wachsgelbe Tepel-Bake. Gm. Vo: luta navicula, sp. 118 . Mart. 3 t. 71 f. 770. 2 St.

1920...10 C. Praeputium. Der schmale bunte Kahn. Gm:Voluta navicula, sp. 118. Mart. 3 t. 71 f. 768. 1 St. 1921...11 C. Tranquebaricum. Die Tranquebarische Tepel-Bake. Gm. Voluta praeputium, sp. 124. Chemn. 10 t. 148 f. $1391,92,1 \mathrm{Si}$.

4. Spira carinata. Mit ausgekiehlter Tepel. 1922...12 Jacobinum. Die weissausgekiehlte Tepel-Bake. Gm. Voluta cymbium, sp. 114. Mart. 3 to 70 f. 762-65. 3 Stiich.

1923...13 C. Lagena viatoria. Die Reise-Flasche, eod. 2 St. 1924...14 C. Cucunis. Deṛ Thürbis, eod. Mart.765. 2 St.

\section{Lade 82.}

1925...15 C. Cochlcar Neptuni. Der Neptunus-Löffel. Gmel. Voluta cymbium, sp. 114. Mart. 3 t. 70 f. 762,63 . \& Stück.

1926...16 C. Patera. Die Schale. Gm. Voluta Neptuni, sp. 117. Mart. 3 t. 71 f. 767. 3 St.

1927...17 C. Carinatum. Die ausgekiehlte Tepel-Bake. Gmel. Voluta Neptuni, sp. 117. Mart. 3 t. 71 f. 767. 2 St.

5. Spira detrita. Mit abgestumpfter Tepel. 1928...18 C. Lineatum. Die liniirte Tepel-Bake. Gm.' Voluta persicula sp. $29 \beta_{\text {s }}$ Mart. 2 t. 42 fo 419,20 . 5 St. 
1929...19 C. Lineata - punctatunt. Die liniirte und punctirte Tepel-Bake. 3 St.

1930...20 C. Interruptum. Die Tepel-Bake mit abgesetzten Linien. 1 St.

1931...21 C. Guttatum. Die besprengte Tepel-Bake. Gmel. Voluta persicula, sp. 29 \%. Mart. 2 t. 42 f. 421.2 St. 1932...22 C. Achatinum. Die Agat Tepel-Bake. Gmel. Bull cyprae, sp. 23. Knorr 6 t. 4 f. 5. 4 St.

1933...23 C. Porphyreum. Die Porphir Tepel-Bake, eod. Martini 2 t. 65 f. $725,27.2$ St.

1934...23 a - Mart. 2 t. 65 f. 728, 29. 2 St.

1935...24 C. Cylindraceum. Die Cylinder Tepel-Bake, eod. 1 Stück.

-1936..25 C. Aurifiácum. Die orange Tepel-Bake. Gm.jBulla cyprae, sp. 23. 2 Sto

\section{Bivalves. Zweyschaaligte.}

\section{Lade 1.}

\section{Solen. Die Messer-Schaalen.}

1...1 S. Siliqua. Die Schote. Gm. Solen siliqua, sp. 2. Chemn.6 tab. 4 fig. 29. Knorr Verg. 6 t. 7 f. 1. 3 St.

2..22 S. Ensis. Das Schwerdt. Gm. Solen ensis. sp. 3. Ch.6 tab. 4 fig. 30. Argenv. Conch. tab. 24 fig. L. 2 St.

3...3 S. Gladius. Der Degen. Gm. Soleñ vagina, sp. 1. Chemn.6 t. 4 f. $2 \% .10 \mathrm{St}$.

4...4 S, Vagina. Die Scheide. Gmel. Solen vagina, sp. 1 Chemn. 6 t. 4 f. $26-28$. Knorr Verg. 1 t. 28 f. 3. 3 St.

5...5 S. Silicula. Dàs Schötchen. Gm. Solen vagina, sp. 1. Favanne p. LV: fig. B. 2. 1 St.

6..6 S. Dolichos. Die gelbe Strahl-Schote, dickschaligt und viel ähnliches von folgender No. 2 St.

7...7 S. Nicobaricus. Die nicobarische Schote. Gmel. Solen maximus, sp. 15 . Chemn. 6 t. 5 f. 35.3 St.

8...8 S. Strigilatus. Die rosenrothe Schote. Gm. Solen strigilatus, sp. To Chemn, 6 to 6 f: 41 , 42. 4. St. 
9...9 S. Lenticula. Die linsenförmige Schote. Gmel. Solen minimus, sp. 14 . Chemn. 6 t. 5 f. 81 a. b. 2 St.

10...10 S. Legumen. Die Hülfe. Gm. Solen legumen, sp. 4. Chemn. 6 t. 5 f. $32-34.3$ St.

11...11 S. Rradiatus. Die gestrahlte Schote. Gmel. Solen radiatus; sp. 6. Chemn. 6 t. 5 f. $38-40.7$ St.

12...12 S. Glycimeris. Die bäuchige Klaffmuschel. Gm. My glycymeris, sp. 1\%. Chemn. 6 t. 3 f. 25. 1 St.

\section{Lade 2.}

\section{Laternula. Die Leuchte.}

13...1 L. Truncata. Die abgestumpfte Lenchte. Gmel. Mya truncata, sp. 1. Chemn. 5 tab. 1 fig. 1-4. Gualt. test. tab. 91 f. D. 2 St.

14...2 I. Anatina. Die Horn-Leuchte. Gm. Solen anatinus, sp. 8. Chemn. 6 tab. 6 fig. 46-48. Rumpf mus. tab. $75 \mathrm{fig}$. O. $3 \mathrm{St}$.

\section{Vulsella. Der IKneiper.}

15...1 V. Major. Der grosse Bart-Knciper. Gm. Mya vul. sella, sp. 6. Chemn. 6 tab. 2 fig. 10, 11. Knorr Verg. 5 t. 2 f. $1-3.1$ St.

16...2 V. Minor. Der lileine Bart-Kneiper. Gm. Mya vule sella, sp. 6. Chemn. 6 t. 2 f. 8, 9. 5 St.

\section{Lithophaga. Der Steinbohrer.}

1\%...1 L. Mytuloides. Der muschelartige Steinbohrer. Gm. Mytilus lithophagus, sp. 6. Chemn, 8 tab. 82 fig. 729, 30. 4 St.

18...- - - Der schwarzbraune Steinbohrer. 1 St.

\section{Mya. Die Miesmuschel.}

19...1 M. Pictorum. Die Mahlermuschel. Gm. Mya pictorum, sp. 9. Chemn. 6 tab. 1 fig. 6. 8 St. wovon= $=3$ abgezogen.

20..2 M. Margaritifera. Die Perlmuschel. Gm. Mya ma2: garitifera, sp. 4. Chemn. 6 t. - f. 5.5 Sto

-..2 a. dito polirt, mit geschnittenen Figuren. 1 St.

21...3 M. Perlata. Die Perlenträgerin. 1 St: 


\section{Musculus, Die Muschel.}

22...1 M. Cygrneus. Die grosse grüne Muschel. Gm. Mytilus cygneus, sp. 15 . Chemn. 8 t. 85 f. 762.3 St.

23...2 M. Anatinus. Die grüne Miesmuschel. Gm. Mytilus, anatinus, sp. 16. Clemn. 8 tab. 85 fig. 763.2 St.

24...5 M. Compressus. Die Maler Strohmmuschel. 3 St. alle dẹf.

\section{Lade 3.}

25...4 IM. Discors. Die queergestreifte Miesmuschel, die platte. Gm. Mytilus discors, sp. 21. Chemn. 8 tab. 86 fig. 767. 1 St.

25...5 M. Novae Zeelandiae. Die Neuseeländische Miesmuschel, die bäuchigte. Gm. Mytilus discors, sp. 21. Ch. 8 tab. 86 fig. 768 . Naturforscli. 10 tab. 1 fig. 8.4 St. davon drei in der Haut.

27...6 M. Modulaides. Die gestreifte Miesmuschel, Gmel. Mytilus modiolus, sp. 14 . Ch. 8 t. 85 f. 760. 15 St: $28 \ldots-\infty-3$ St.

29...7 M. Papuanus. Die papuanische Miesmuschel. Gmcl. Mytilis modiolus, sp. 14 . Chemn. 8 t. 85 f. 758. 1 St. 30...8 M. Modulus. Die Modelmuschel. Gm. Mytilus modiolus, sp. $14 \%$. Lister t. 1057 f. 5. S St.

\section{Mytilus. Die Spitzmuschel.}

1. Glaberrimoe. Glatte.

31...I M. Ungulatus, Die blaue Spitzmuschel, Gm. Mytilus ungulatus, sp. 12. Chemn. 8 tab. 84 fig. 756 . 5 St.

$15731 a_{0}-\alpha-1$ St.

32...2 M. Variegatus. Die bunte Spitżmuschel. Gm. Mytilus versicolor, sp. 30 . Chemn. 8 tab. 84 fig. 748 . S St.

33...3 M. Violaceus. Die violenblaue Spitzmuschel. Gmel. Mytilus edulis, sp. 11 . Chemn. 8 tab. 84 fig. 750, 51. 3 Stück.

$34 . . .-\alpha-2$ St.

35...4 M. Edulis. Die Essmuschel. Gmel. Mytilus edulis, sp. 11. 1 St.

86...- - - monströss: 1 St.

37...5 M. Magellanicus. Die magellanische Spitzmuschel, Gm. Mytilus ungulatus, sp. 12. Chemn. 8 tab. 83 fig. 733. 1 Sto 
38...6 M. Pictus. Die buntschäckigte Spitzmuschel. Gmel. Mytilus afer, sp. 28. Chemn. 8 t. 83 f. 739. 3 St. $39 . . .-\alpha-$ Chemn. 8 t. 83 f. 740. 1 St. $40 \ldots-\beta-1$ St.

11...5 M. Roseus. Die rosenrothe Spitzmuschel. 1 Sto

\section{Mytilus. Die Spitzmuschel.}

\section{Sulcatce. Gerillte.}

42...8 M. Sulcatus. Die gerillte Spitzmuschel. Gm. Mytilus bidens, sp. 13. 4 St.

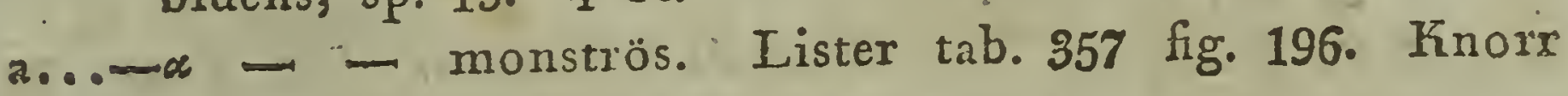

Vergn. 4 t. 30 f. 3.3 St.

13...9 M. Nicobaricus. Die nicobarische Spitzmuschel. Gn.

Mytilus bilocularis, sp. 8. Chemn. 8 t. 82 f. 796 . 4 St. 14...10 M. Purpureus. Die Purpurmuschel. 1. St.

45...11 M. Citrinus. Die citronenfarbene-Spitzmuschel. Gm. Mytilus exustus, sp. 9. Chemn. 8 t. 84 f. 754. 1 St. a...12 Eine bis jetzt linbehannte Pergament Miesmuschel. 1 Stück.

\section{Pharetra. Der Köcher.}

26...1 P. Monoculoides. Die Pfeilmuschel. Chemn. 10 t. 172 f. 1675-77. Naturforscher 22stes St. tab. 3 fig. A-E. p. 23. $1 \mathrm{St}$.

\section{Lade 4 \& 4 a.}

\section{Pinna. Der Schinken.}

47...1 P. Gigas. Der grosse Schinken. Gm. Pinna rotundata, sp. 5. Chemn. 8 t. 93 f. $78 \%$. 1 St.

48...2 P. Bicolor. Der zweifärbige Schinken. Gm. Pinna bi० color, sp. 19. Chemn. 8 t. 90 f. 780.1 St.

$49 . .2$ * P. Striata. Der gestreifte Schinken. 1 St.

50...3 P. Gubernaculum. Der geräucherte Schinken. Gmel. Pinna vexillum, sp. 15. Chemn. 8 t. 91 f. 783. 1 St,

51.... P. Inflata. Der angeschwollne Schinken. 1 St。 52...5 P. Vitrea. Der dursichtige Schinken. Gm. Pinna pecs timata, sp. 2 var. $\beta$. Cheman. 8 t. 87 fo 771. S St. 
53...6.P. Rudis. Der schwarze Schinken. Gm. Pinna rudis, sp. 1 ß. Chemn. 8 t. 88 f. 774 . 1 St.

51...7 P. Ferruginea. Der rothe schuppige Schinken. Gmel. Pinna rudis, sp. 1 ß. Chemn. 8 t. 88 f. 773.1 St.

55...8 P. Violacea. Der blaue Schinken. 1 St.

56. .9 P. Nobilis. Der kleine schuppige Schinken. Gmel.

Pinna nobilis, sp. 3. Chemn. 8 t. 89 f. 775. 2 St.

57...10 P. Pectinata. Der geflammte Schinken. Gm. Pinna nobilis, sp. 3. Chemn. 8 t. 89 f. 775.2 St.

58...11 P. Pernula. Der gespickte Schinken. Gm. Pinna no. bilis, sp. $3 \%$. Chemn. 8 tab. 92 f. 785. Friorr 2 tab. 26 fig. 2. 2 St.

59...12 P. Elongata. Der schmale Schinken. Gm. Pinna ru. dis, sp. 1. Chemn. 8 t. 89 f. 775. 1 St:

\section{Lade 5.}

\section{Beguina. Die Beguin-Nonne.}

60...1 B. Nephritica. Die nierenförmige Beguin-Nonne. Gm: Chama cordata, sp. $8 \beta$ reniformis. Chemu, 7 tab. 50 fig. 502, 3. 4 St.

\section{Mantellum. Das Mäntelchen.}

61...1 M. Excavatum. Die Auster des Fabricius. Gm, Ostiea excavata, sp. 98. Chemn. 7 t. 68 f. 654.1 St.

62...2 M. Inflatun. Der bäuchigte halbe Mantel. Gm. Ostrea inflata, sp. 70. Chemn.7 t. 68 f. 649. 5 St.

68...3 M. Scabrum. Die Reibe. Gm. Ostrea glacialis, sp. 96. Chemn. 7 t. 68 f. $652,53.5$ St.

64...4 M. Lima. Die Feile. Gm. Ostrea lima, sp. 95. Ch. 7 tab. 68 fig. 651.6 St.

\section{Lade 6.}

\section{Chlamys. Der Mantel:}

1. Glabroe. Glatte.

* Striatae. Gestreifte.

65...1 C. Cinnabarina. Dex zinoberrothe Mantel. Gn. Ostrea istandica, sp. 55 . Chemn. 7 t. 65 f. $615,16.9$ St.

66...2 C. Islandica. Der islündische Mantel. eod. \& St. 
**. Sulcatea. Gefurchte.

6\%...s C. Norwegica. Der norwegische Mantel. Gm. Ostrea citrina, sp. 62. Cliemn. 7 t. 65 f. $618.13 \mathrm{St}$.

$68 \ldots-\infty-3$ St.

$69 . . . \beta-1$ - St.

$70.1 .-\gamma-1 S_{t}$.

71...3* C. Solaris. Der gelbe Strahlmantel. Gmel. Ostrea glabra, sp. 50 . von Born t. 6 f. 4. 2 St.

72...4 C. Septentrionalis. Der noidische Mantel. Gm. Ostrea glabra, sp. $50.10 \mathrm{St}$.

73...5 C. Tranquebarica. Der tranquebarische Mantel. Gma Ostrea tranquebarica, sp. 67. Chemn. 7 tab. 67 fig. 647. 4 Stück.

$74 \ldots-\infty-1 S t_{0}$

\section{Lade 7.}

75..6 C. Cruor. Der Jlutrothe Mantel. St.

76...7 C. Purpurascens. Der Purpurrothe Mantel. Fnorr 5 tab. 12 fig. 5. 1 St.

77...8 C. Purpurata. Der bläuliche Purpur-Mantel. 5 St. 78...9 C. Testudinaria. Der Schildpatmantel. i St.

79...10 C. Brunnea. Der braune Mantel. Knorr 5 Th. tab. 12 fig. 4. 3 St.

80...11 C. Gibba. Der rothe bäuchigte Mantel. Gm. Ostrea gibba, sp. 52. Chemn. 7 tab. 65. f. 619, 20. 2 St.

81. 12 C. Radula. Die bräunliche Jägertasche. Gm. Ostrea radúlá, sp. 11. Cliemn. 7 t. 68 f. $599,600.3$ St.

82...I3 C. Venatoria. Die kleine Jägertasche. 3 Șt.:

8s...1 1 C. Superba: Die vortrefliche Jägertasche. $1 \mathrm{St}$

*** Plicatae. Gefaltete.

85...16 C. Mediterranea. Der Mantel aus den mittelländi. schen Meere. $12 \mathrm{St}$.

86...17 C. Citrina. Der citrongelbe Mantel. 1 St.

87...18 C. Crocea. Der safranfarbene Mantel. Gm. Ostiea crocea, sp. 78. 1 St.

88...19 C. Miniata. Der blassrothe ManteI. 1 St.

89...20 C, Amaranthina. Der amarantfarbene Mantel. $1 \mathrm{St}$,

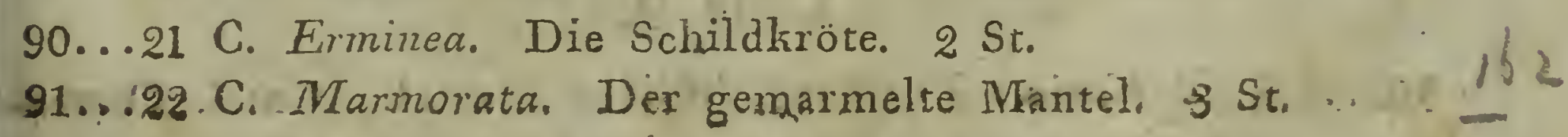


92...23 C. Rubiginosa. Der rostfarbene Mantel. Gm. Ostrea glabra, sp. 50. Clemn. 7 t. 67 f. 643.2 St.

93...24 C. Fuliginosa. Der braungelbe Mantel. eod. 1 St. 94...25 C. Punctulata. Der punctirte Mantel, eod. 3 St. 95...26 C. Intertexta. Der gewirkte Mantel. eod. 1 St. 96...27. C. Episcopalis. Der bischöfliche Mantel, eod. I St. 97...28 C. Tentorialis. Der buntschächigte Mantel, eod. 2.St. 98...29 C. Castrensis. Der Feldmantel. eod. 2 St. 99...30 C. Capucina. Der Capuziner-Mantel. eod. 3 St.

\section{Lade 8.}

100...31 C. Cornéa. Der buntfarbige Mantel. Gmel. Ostrea glabra, sp. 50. Chemn. 7 t. 67 f. 642.11 St.

\section{Chlamys. Der Mantel. \\ 2. Stabriusculoe. Rauhe. \\ * Nodosae. Knotige.}

101...32 C. Nodosa. Die Korall-Dublette. Gm. Ostrea nodosa, sp. 43. Chemn. 7 t. 64 f. 609, 10. Knorr Verg, 1 t. 5 f. 1 \& 4 t. 13 f. 5.5 St.

** Squamatae. Geschupte.

102...s3 C. Pallium. Der Herzogsmantel. Gm. Ostrea paI. lium, sp. 40. Chemn. 7 tab. 64 fig. 607. Knorr V. 2 t. 21 f. 1, 2. $11 \mathrm{St}_{\text {。 }}$

103...- $\alpha-1$ St.

104...34 C. Sulphurea. Der schwefelgelbe Mantel. Gm. Ostr. sulphurea, sp. 64. Chemn. 7 t. 66 f. 629. 1 St.

105...35 C. Cardinalis. Der Cardinalsmantel. Gmel. Ostreá porphyrea, sp. 65. Chemn. 7 t. 67 f. 632.4 St.

105...35 C. Aurantiaca. Der orange Mantel. Gm. Ostrea all. rantia, sp. 37. 17 St.

107...37 C. Gusio. Der kleine Mantel. Gmel. Ostrea gusio, sp. 49: 1 St.

108... - - Chemn. 7 t. 49 f. 635 . 1 St.

\section{Lade 9.}

309...38 C. Varia. Der veränderliche Mantel. G Gel. Ostrea varia, sp. 48. Clıemn, 7 to 66 f. 633 . 14 St. 
110...39 C. Incerta. Der buntsahäckige ManteI. $1 \mathrm{St}$.

111...40 C. Nebulosa. Der wolkigte Mantel. 3 St.

112...41 C. Striata. Der gestreifte Mantel. 1 St.

113...42 C. Lingua-Felis. Die Katzenzunge. Schröters Einla in die Conch. 3 B. Seite 328 N. 15. Chenn. 7 tab; 66 fig. 630.1 St.

114...43 C. Incarnata. Der blutrothe Mantel. Gmel. Ostrea incarnata, sp. 83.2 St.

115...44 C. Pseudamusium. Der unächte Compas. 2 St.

116...45 C. Papyracea. Der dünne Papiermantel. Gm. Ostrea vitrea, sp. 66. Chemn. f. 637 a. 2 St.

\section{Pecten. Die Kamm-Muschel.}

117...I P. Maxima. Die grösste Kamm-Muschel. Gm. Ostr. maxima, sp. 1. Chemn. 7 t. 60 f. 585. Knorr Vergn. 1 t. 14 f. 12 \& 2 t. 14 f. $1-3.1$ St.

118...2 P. Jacobaea. Die Jacobsmuschel. Gm. Ostrea jacobaea, sp. 2. Chemn. 7 t. 60 f. $588,89.2$ St.

119...\& P. Dubius. Die Kleine Kamm-Muschel. eodo \& St. 120...4 P. Pictus. Die bunte Kamm-Muschel, eod. 1 St. 121...5 P. Ziczac. Die ziczac Kamm-Muschel. Gm. Ostrea ziczac, sp. 3. Chemn. 7 t. 61 f. 590, 91. 4 St. 122...- - - eod. Chemn. t. 61 f. 592. 2 St. 123...6 P. Politzs. Die geglättete Kamm-Muschel. 2 St.

\section{Lade 10.}

\section{Amusium. Die Compas-Dublette.}

124...1 A. Plenronectes. Die Compas-Doublette. Gm, Ostrea pleuronectes, sp. 6. Chemn. 7 tab. 61 fig. 595. Knorr Vergn. 5 t. 20 f. 3, 4. 1 St.

125...2 A. Japonicum. Die Japanische Compas-Dublette. Gm. Ostrea japonica, sp. 8. Chemn. 7 t. 62 f. 596. 4 St.

126...3 A. Magnetzcum。 Die gräulichröthliche Compas-Dublette. $\mathrm{Gm}$. Ostrea pleuronectes, sp..6. I St.

127...4 A. Lorentzianum。 Die Lorenzische Compas-Dublette. Gm. Ostrea Laurentii, sp. 7\% Chemn. 7 tab. 61 fig. $593,94.2$ St.

128...5 A. Testudinarium。 Die Schildpatfarbene Compas-Dublettọ. 1 St. 


\section{Lade 11. \\ Ephippium. Der Sattel.}

Säinmtlich selten.

*199 . I ! E. Polcnicum. Der polnische Sattel. Gm, Anomia sella, sp. 2\%. Chemn. 8 t. 79 f. 714. Knon: Vergn. 4 t. 18 f. 2 \& 5 t. 29 f. 1.1 St. sehr schon.

*150..2 E. Placenta, Der Kuchen. Gmel. Anomia placenta, sp. 26. 1 Śt.

*131..s E. Transparens. Der durchsichtige Sattel. Gm. Anomia placenta, sp. 26. Chemn. 8 t. 79 f. 716. $1 \mathrm{St}$.

*132..4 E. Anomia. Die braune Transparent-Musclel, oder der Kleine papierne Englische oder Polnische Sattel. Chemn. 8 t. 79 f. 715. Knor Vergn. 2 tab. 24 fig. 1. Rumph. Amboin. t. 47 f. B. Ostreum placentiforme. 1 Stüchio.

\section{Calopodium. Der Leisten.}

*133. 1 C. Albidum. Der weisse Leisten. Gm. Telliná inaequivalvis, sp. 23. Chemn. 6 t. 11 f. 105 a.b.c.d. 1 St.

- Pinctada. Die Perlmutter.

1. Latce. Perlmutter-Schale.

134...1 P. Margaritifera. Die Perlmutter. Gmel. Mytilus margaritiferus, sp. 4 . Chemn. 8 t. 80 f. 717-21 a. b. Knorr Vergn. 2.t. 25 f. 1. 3 St. eine davon ist polirt. 125..2 P. Foliacea. Die blättrige Perlmutter. Gm. Mytilus margaritiferus, sp. 4. Knorr Verg. A t. 18 f. 1 St.

136...s P. Castanea. Die castanienbraune Perlmutter. Gmel. Mytilus margaritiferus, sp. 4. 1 St.

13\%...4 P. Imbricata. Die 'schuppige Perlmitter. Gm. Myti. lus margaritiferus, sp. 4. Chemл. \& f. 719. Fnorr Verg. 2 t. 25 fig. 2, s. 4 St. wovon 1 St. halb.

\section{Lade 12.}

\section{Pterigiata. Vögelchen.}

*138..5 P. Penguir. Der Penguin. Gmel. Mytilus hirundo, sp. $22 \delta$. 'Chemn. 8 t. 81 fig: 72s. Knorr Vergn. 6 t. 2 fig. 1. 1 St. 
*139..6 P. Colýmbus. Die grosse Schwalbe. Gmel. Mytilus hirundo, sp. 22. Chemn. 8 t. 31 fig. 723. 1 St. sp. 2z. Chemn. 8 t. 81 fig. 725. $3 \mathrm{St}$.

*141..8 P. Brunea, Dãs braune Vögelchen. Gmel. Mytilus hirundo, sp. 22. $1 \mathrm{St}$.

*142..9 P. Apus. Die Geierschwalbe. Gm. Mytilus hirundo, sp. 22. 1 St. defect.

\section{Pinctada. Die Perlmutter.}

3. Elongatce. Hammer. Selten.

*143..10! ! P. Malleus. Der Hammer. Gm. Ostrea malleus, sp. 99. Chemin. 8 t. 70 f. 655 . Knorr 3 t. 4 fig. 1 Lister t. 219 fig. 54 . 3 St. Selten und schön.

*144..11 P. Regula. Das Lineal, Gm. Ostrea vulsella, sp.100. Chemn. 8 t. 70 fig. $65 \%$. 5 St. Selten.

\section{Isogonum. Das Winkelmaass. Selten.}

*145..1 I. Norma. Das violet und weisse Iineal. Gm. Ostrea Isognonum, sp. 125. \% Chemn. 7 t. 59 fig. 582. 1 St. *145..2 I. Gnomon. Der violette Winkelhaken.: Gm. Ostrea Isogonum, sp. 125i. $\delta$. Chemn. 7 t. 59 f. 583, 84. 1 St. ${ }^{*} 147 . .3$ Is Scapula. Das Schulterblatt. Gm. Ostrea ephippium. sp. 126. Chemn. 7 t. 58 fig. 576. Knorr 6 t. 21 fig, 1. 2 Stiuck.

148...4 I. Marsupiale. Die Tasche. Gm. Ostrea ephippium, sp. 126. Chemn. 7 t. 58 fig. $57 \%$. $1 \mathrm{St}$.

149...5 I. Crenulatun. Das gekerbte Schulterblatt.? Gm. Ostrea ephippium, sp. 125. 1 St.

150...6 I. Alaphasiani。 Der Fasanenflügel. Gm. Ostrea picta, sp. 12\%. Chemn。 7 t. 58 fig. 575. 2 St.

151...7 I. Semiauritum. Der olırförmige Flügel. Gm. Ostrea perna, sp. 124. Chemn. 7 t. 59 fig. 579, 80. S St.

\section{Lade 13."}

\section{Lopha. Der Hahnenkamm.}

152...1 L. Crista Galli. Der Hahnenkamm. Cmel. Mytilus crista galli, sp. 1. Cliemn. 8 t. 75 fig. 683. $7 \mathrm{St}_{\text {。 }}$ 
153...- $\alpha-$ - Chemn. 8 t. 75 fig. 684. 2 St.

154...2 L. Hyotis. Der doppelte Hahnenkamm. Gm. Mytilus hyotis, sp. 2. Chemn. 8 t. 75 fig. 685. Argenv. zoomorph. t. 12 fig. F. 2 St.

155...3 L. Frons. Das gefaltete Blatt am hölzern Stiel. Gm. Mytilus frons, sp. 3. Chemn. 8 t. 75 fig. 686. Knorr Verg. 4 t. 8 fig. 3. 1 St.

156...4 L. Folium. Das Lorbeer-Blatt. Gm: Ostrea folium, sp. 103. Chemn. 8 t. 71 fig. 662-66. Knorr Verg. I t. 23 fig. 2. 1 St.

157...5 L. Cornu Copiae. Das Füllhorn. Gm. Ostre corna copiae, sp. 114. Chemn. 8 t. 74 fig. 679. v. Born Caes. Vindab. test. to 6 fig. 11, 12. $2 \mathrm{St}$,

$158 \ldots-\infty-1$ St.

159...6 L. Theca. Die Dose. Chemn. 8 t. 73 fig. 675. 3 St. $160 \ldots-\alpha-1 \mathrm{St}_{\text {. }}$

161...7 L. Plicatella. Der dregechige grŭne Hahnenkamm. 3 Stück.

\section{Lade 14. \\ Ostrea. Die Auster.}

162...1 0. Rhinantoides. Die blaue gediuchte Auster. 2 St. 163...2 O. Virginiana. Der Neger-Kahn. Gm. Ostrea virginica, sp. 113. Chemn. 8 t. 73 fig. 67\%. S St.

164...3 O. Praeadamitica. Die Sündfluths-Auster. Gm, Ostrea sinensis, sp. 108. Chemn. 8 t. 72 fig. 668. 1 St.

165...4 O. Lineata. Die liniirte Auster, 4 Stück, wovon $2 S t$. doppelt.

166...5 O. Bilineata, Die zweimal liniirte Auster. Gm. Ostr. edulis, sp. $105 \delta$. Chemn. 8 t. 71 f. 660.4 St.

167...6 O. Stellata. Die Stern-Auster. $4 \mathrm{St}$.

*168.7 O. Pedum. Der Schäferstab. Aeusserst rar. Favanne Pl. 80 fig. K. 1 St.

\section{Lade 15.}

169...8 0. Edulis. Die essbare Auster.' Ostrea edulis, sp. 105. s. Chemn. 8 tab. 71 fig. 661. Chemn. 8 tab. 74 fig. 682. $23 \mathrm{St}$. 
170...9 0. Elongata. Die länglichte Auster. eod. ל. Chemn. 9 t. 116 f. 995, 93. 3 St.

171...10 O. Silicula. Die kleine länglichte Auster. eod. 1 Pt.

Lade 16 ,

\section{Mactra. Die Korb-Muschel.}

172...1 M. Glauca. Die gestrahìte Korbmuschel. Gm. Mactrà glauca sp. 20. Chemn. 6 t. 23 f. 232, 33. 4 St. 173...2 M. Maculata. Die gefleckte Korbmuschel. Gmel. Mactra maculata, sp. 16. Chemn. 6 t. 21 f. 208, 209. I Stück.

174...4 M: Corallina. Die blassiöthliche Korbmuschel. Gm. Mactra corallina, sp. 9. Chemn, 6 tab. 22 fig. 218, 19. 2 Stück.

175...4 M. Lactea. Die milchweisse Korbmuschel. Gmel. Mactra lactea, sp. 10. Chemn, 6 t. 22 f. 220, 21. 3St. 176...5 M. Violacea. Die blane Korbmuschel. Gm. Mactra violaceá, sp. 18. Chemn. 6 t. 22 f. 213, 14. Knorì Vergn. 6 t. 5 f. 2. 6 St:

177...6 M. Stultorum. Der Narrenkorb. Gm. Mactra stulto. rum, sp. 11. Chemn. 6 t. 23 f. 224-26. Tnorr V. 6 t. 5 f. 1.2 St.:

178...7 M. Striatula. Der gestreifte Khorb. Gm. Mactra stria* tula, sp. 4. Chemn. 6 t. 21 f. 205, 6. Khnorr Vergns 6 t. 34 f. 1 . 2 St. $_{\text {. }}$.

179...7 * M. Bicordata. Der herzförmige Korb. $2 \mathrm{St}_{\text {。 }}$ 180...8 M. Pellucida. Der durchsichtige Korb. Gm. Mactra plicataria, sp. 2. Chemn. 6 t. 20 f. 202-4. 5 St. 181...9 M. Spengleri. Der Spenglerische Korb. Gm. Mactra. Spengleri, sp. 1. Chemn. 6 t. 20 f. $199-201.1$ St. 182...10 M. Rugosa. Der runzliche Korb. 'Gm. Mactra ги. gosa, sp. 23. Chemn. 6 t. 24 f. 236. 2 St.

193...11 M. Lutraria. Der Fischkorb. Gm. Mactra Lutraria, sp. 14. Chemn, 6 t. 24 f. $240,41.1$ St,

\section{Lade 17.}

\section{Tridachnes, Die Nagel-Muschel.}

184...1 T. Maxima. Die grösste Nagelmuschel. Gm. Chama gigas, sp. 2. Chemn, 7 th, 49 f. $495.3 \mathrm{St}_{\text {。 }}$ 
185..- - Ckemr. 6 t. 49 f. $495.2 \mathrm{St}$.

186...2 T. Noae. Die Vater Noahmuschel. Gm. Chama gi.

gas, sp. 2. Chemn. 7 t. 49 f. 494. St:

$187 . . .-c$ - -2 St.

188...3 T. Gigas. Die grosse Nagelmuschel. Gmel. Chama gigas, sp. 2. 1 St.

$189 . .+-\infty-1 \mathrm{St}$.

190...4 T. Imbricata. Die gedechte Nagelmuschel. GmelChama gigas, sp. 2. 1 St.

191...-u - - Chemn.7 fig. 492, 99. $6 \mathrm{St}_{\text {: }}$

$192 \ldots-\beta-2 S t$.

$193 \ldots-\gamma-1 \mathrm{St}$.

$194 \ldots-\delta-\delta s t$.

1942..-is - 1 St.

195...5 T. Derasa. Die glatte Nagelmuschel. Gmel. Chama .gigas, sp. 2. ß. Cheman, 7 t. 49 fig. 497. \& St.

\section{Lade 18.}

196..66 T. Hippopus. Der Pferdelıu. Gm. Chama hippopus,

sp. 3. Chemn. 7 t. 50 fig. $498,99.9$ St.

197...7 T. Ungulà. Der längliçhte Pferdehuf. Gmel. Chama hippopus, sp..3. Chemn. 7 t. 50.fig.498. 99. 1 St.

198...8 T. Arcinella. Der stachligte Pferdehaf. Gm. Chama arcinella; sp. 14. Mart: fig. 522, 23. Knorr Verg. 6 tab. 36 fig. 1 , 2. 3 St.:

\section{Lade 19. \\ Tuceta. Die Pastete.}

199...1. T. Pilosa. Die grosse Dichmuschel. Gmel. Arca pilosa, sp. 36. Chemn. 7 t. 57 f. 565, 66. Knorr Verg. 2 t. 23 fig. 6 t. 12 f. 4. 1 St.

200..2 T. Glicymeris. Die röthliche Pastete. Gmel. Arca glicymeris, sp. 35. Chemn. 7 t. 57 fig. 564. Know2 V.1 t. 21, fig: 4 \& 6 t. 14 fig. S. $2 \mathrm{St}$.

201...3 T. Leutiginosa. Die bunte Pastete. Gn. Arca undata, sp. 32. Knorr Verg. 6 t. 14 f. 4. 2 St.

202...4 T. Undata. Die gewellte Pastete. Gm. Arca undata, sp. 32. Chemn, 7 t. 57 fig. 560.2 St. und 1-halbe. 203...5 T. Inflata. Die bäuchigte Pastete. Gm. Anca pilosa. sp. 36.1 St. 
204..6 T. Sinnata. Die gestrahlte Pastete. Gm. Arca angulosa, sp. 41. Chemn. 7 t. .57 f. 567. 1 St.

205...7 T. Mytiloides. Die muschelförmige Pastete. Gmel. Arca glycymeris, sp. 35.1 St.

206...8 T. Pectunculus. Die gerillte Pastete. Gm. Arca pectunculus, sp. 33. Chemn. 7 t. 59 f. 558, 69. 4 St.

\section{Arca. Die Arche.}

1. Pectunculoidea. Kammförmige.'

20\%...1 A. Cucullata. Die Mönchskappe. Gm. Arca cucullus, sp. 23. Chemn. 7 t. 53 f. 526-28. 1 St.

208...2 A. Antiquata. Das Paquetboot. Gm. Arca antiquata, sp. 16. Chemn. 7. t. 55 f. 548. Knorr Vergn. 1 t. 24 f. $3,-4$. 3 St.

\section{Lade 20.}

209...3 A. Scapha. Die kahnförmige Arche. Gm. Arca anti, quata, sp. 16. 2 St.

210...4 A. Notabilis. Die gezierte Arche. Gm. Acra antiquata, sp. 16. Chemn. 7 t. 55 f. 549.4 St.

211...5 A. Inaequivalzis. Die ungleichschaaligt - bäuchigte Arche. Gm. Arca indica, sp. 2\%. Chemn. 7 tab. 56 fig. 552. $2 \mathrm{St}$.

212...- - - Die gestreckte Dito. 2 St.

213...6 A. Granosa. Die körnichte Arche. Gm. Arca gránosa, sp. 18. Chemr. 7 t. 56 f. 55\% 3 St.

214...7 A. Rhombea. Die rautenförmige Arche. Gm. Arca rilomboidea, sp. 39, Chemn. 7 to 56 f. 553 a. 2 St. 215...8 A. Grandaeva. Die bejahrte Arche. Gm. Arca senie lis, sp. 1\%. Lister Conch. t. 238 f. 72. 1 St.

216...9 A. Senilis. Die alte Arche. Gm. Arca senilis, sp. 1\%. Chemn. 7 t. 56 f. 55 t-56. $5 \mathrm{St}$.

\section{Arca. Die Arche.}

2. Verce. Die wahren Archen.

217... 10 A. Noae. Die Arche Noah. Gin. Arca Noae, \$p. Chemn. 7 t. 53 f. $529-31$. 2 St.

$218 \ldots-\infty-\infty$ - S.

$219 \ldots-\beta-\beta S t_{0}$ 
2q0...11 A. Patriarchális. Die uralte Arche. Gm, Arča None, sp. 2. Chemn. 7 t. 53 f. 59 2. 19 St.

.221...12 A. Martini. Die Arche des Martini. Gm. Arca rostrata, sp. 8. Chemn. 7t. 55 f. 550, 51. 3 St.

222...12*A Undata. Die wellenförmige Arche. Gm, Arez rostrata, sp. 8. var. 1 St.

\section{Arca. Die Arche.}

223...13 A. Nivea. Die schneeweisse Arche. Gm. Arca ovath, sp. 6. Cliemn. 7 t. 54 f. 538.1 St.

224... 14 A. Reticulata. Die gefurchte Arche. Gm. Arca reticulata, sp. 25. Chemn. 7 t. 55 f. 540.2 St.

225...15 A. Barbata. Die haarichte Arche: Gm. Arca brrbata, sp. 3. Chemn. 7 t. 54 f. 535.4 St.

225...16 A. Amygdalum tostum. Die gebrannte Mandel. Gm. Arca barbata, sp. $3 \gamma$. Chemn. 7 t. 54 f. 534, 2 St.

227...17 A. Magellanica. Die magellanische Arche. Gmel.

A. magellanica, sp. 24. Chemn; 7 t. 53 f. 539.1 St.

\section{Trisidos, Die gedrehte Arche.}

228...1 T. Arca tortuosa. Die gedrehte Arche. Gmel. Area tortuosa, sp. 1. Chemn. 7 t. 53 f. 524, 25. Knorx V. 1 t. 28 f. 3. 1 St.

\section{Lade 21 .}

\section{Paphia. Die Flügelmuschel.}

229...1 P. Ala avis. Die riesenmässige Venusmuschel. Sehr: selten und ein Exemplar von ausserordentlicher Grüsse, Gm. Venus gigantea, sp. 89. Chemn. 10 tab. 171 fig. 1661. Favanne Concl. t. 49 f. 91.2 St.

230...2 P. Ala papilionis. Der Schmetterlingsflügel. Gm, Vonus rotundata, sp. 134. Chemn. 7 t. 42 f. 441 . Kuorr Vergn. 2 t. 18 f. 4.4 St.

231 ...3 P. Guttulata. Die gefleckte Flügelmuschel. Gm. Venus litterata, sp. $192 \delta$. Litterata conspersa. Chemm. 7 t. 42 f. 438.1 st. 
252...4 P. MTerüz. Die kleine Matte. Gm. Venus Meröe, sp. 22. Chemn. 7 t. 43 f. 450 . 12 St.

$233 \cdot . . \infty-1$ St.

234...- $-\beta-$ Chemn. 7 t. 43 f. 453 . 1 St.

$235 \ldots-\gamma--5 S t$.

$235 \ldots-\delta-1$ - 1 t.

$237 \ldots-8-3 \mathrm{St}$.

238...5 P. Edulis. Die essbare Flügelmuschel. Gm. Venus virginea, sp: 136. Chemn. 7 t. 43 f. $45 \%$. $4 \mathrm{St}$.

239...6 P, Litterata. Die spanische Matte. Gm. Venus litterata, sp. 132. Chemn. 7 t. 41 f. $432-34.5$ St.

240...7 P. Nocturna. Die schwarze Matte. Gm. Venus litterata nebulosa, sp. 132 \&. Chemn. 7 tab. 41 fig. 435. Knorr Vergn. 6 to 14 f. 1. 7 St.

241...8 P. Decussata. Die gestrahlte Flügelmuscliel. Gm. Ve. nus decussata, sp. 135. Ch. 7 t. 43 f. 455, 56. 17 St. 242...9 P. Nebulosa. Die wolkigte Flügelmuschel. I St.

\section{Lade 22,}

\section{Gafrarium. Das Waffeleisen.}

243.:.1 G. Fimbriatum. Das weisse Waffeleisen. Gm. Venus fimbriata, sp. 15. von Born t. 5 f. 4. Chemn. 7 p. 3 vign. 8 \& t. 49 f. 448,49 . Lister t. 355 f. $172 \&$ tab 1056 f. 1. 6 St.

244...2 G. Tumidum, Das bäuchigte Waffeleisen. Gm. Venus pectinata, sp. 78. Chemn. 7 t. 39 f. 4152 St.

245...3 G. Angulatum. Das winkligte Waffeleisen. Gm. Venus pectinata, sp. 78 . Chemn: 7 t. 39 f. 417. Lister t. 312 f. 148 t. 313 f. 149. 5 St.

216...4 G. Depressum. Das piatte Waffeleisen. Gmel. Venus pectinata, sp. \%3. I St.

247...5 G. Cardiodeum. Das eyförmige Waffeleisen. Gmel, Venus pectinata, sp. 78. $1 \mathrm{St}$.

248... 5 G. Costatum. Das gerippte Wafreleisen. Gm. Venus pectinata, sp. 78. $1 \mathrm{St}$.

249...7 G. Pectinatum. Das braune punctirte Waffeleisen. Gm. Venus pectinata, sp. 78. Cliemn. 7 tab. 39 fig. 418 , 419. 2 St.

250...8 G. Tellina. Das buntschäckigte Waffeleisen. Gmel. Tenus reticulata, sp. 26. 1 St. 
251...9 G. Reticulatune. Das gegitterte Waffeleisen. Gm. Tenus reticulata, sp. 26. Chemn. 6 tab. 36 fig. $382-385$. 2 Stück.

252...10! G. Venusium. Das dreieckigte Waffeleisen mit Zacken. 1 St.

253...11 G. Didymum. Das rauhe Waffeleisen. $2 \mathrm{St}$. 254...12 G. Levigatum. Das geglättete Waffeleisen. 1 St.

\section{Cytherea. Das Venusbecken.}

\section{Depressce. Platte.}

255... I C. Lavacrum. Das Waschbecken. Gm. Venus grenu. lata, sp. 33. von Born t. 4 f. 5 . 5 St.

256...2 C. Nivea. Das schneeweisse Venusbechen. Gm. Ve. nus tigerina, sp. 69: 1 St.

25\%...3 C. Tigerina. Das gesprenkelte Venusbecken. Gmel. Venus tigerina, sp. 69. Chemn. 7 tab. 37 fig. 390, 91. 6 Stück.

258. $\therefore-\alpha-\infty$ - 1 St. polirt und geschliffen.

259...4 C. Concentrica. Das gestreifte Venusbecken. Gmel. Venus concentrica, sp. 82. Lister t. 261 f. 91. Chena. 7. t. 37 f. $392 . \quad 1$. St.

260...5 C. Scripta. Das ziczacartig gezeichnete Venusbecken. Gmel. Venus scripta, sp. 79. Chemn. 7 t. 40 f. 422 , $23 \& 24.2$ St.

261...- - - Chemn. 7 t. 40 f. 420. 3 St.

262...6 C. Corrugata. Das gerunzelte Venusbecken. Gmel. Venus scripta, sp. 79 \%. Chemn. 7 t. 39 fig. 410, 11. von Born t. 5 f. $\%$. $5 \mathrm{St}$.

263...7 C. Exsoleta. Das veraltete Venusbecken. Gmel. Venus exsoleta, sp. $75 . \quad$ Chemn. 7 t. 38 f. 404 . Gualt. test. t. 75 f. D. 2 St.

264...8 C. Obsoleta. Das reraltete bunte Bechen. Gm. Histrio, sp. 35. Chemn. 7 t. 38 f. 407. 2 St.

205...9 C. Albida. Das weisse Venusbecken. Gmel. Venus. exoleta, sp. $75 \cdot 1$ St.

2jo...10 C. Excisa. Das ausgeschnittene Venusbecken. Gm.

Venus sinliata, sp. 76 . Chemn. 7 t. 38 f. $400,401$.

1 Stück.

267...1I C. Detrita. Das abgenutzte Venusbecken. 1 St. 


\section{Cytherea. Das Venusbecken.}

2. Tumidce. Bäuchigte.

268...12 C. Puerpera. Das Kugelbecken, die Kindbetterin. Gm. Venus puerpera, sp. 28. Chemn. 6 t. 36 f. 388, 89. Knorr Vergn. 6 t. 10 f. 1. 4 St.

269...13 C. Rugosa. Das starkgerunzelte Venusbecken. Gm. Venus rugosa, sp. 31. Chemn. 6 t. 29 f. 303. 1 St.l 270...14 C. Senilis. Das alte Venusbecken, der Pfirschkern. Gm. Venus Verucosa, sp. 6. 4 St.

271...15 C. Verucosa. Das warzige Venusbecken, Gm. Venus verucosa, sp. 6. Chemn. 6 t. 20 f. 299, 300. 3 St. 272...15 C. Biradiata. Das doppelte gestrahlte Vennsbecken. Chemn. 7 t. 38 f. 405.1 St.

273...17 C. Brunnea. D.as braune Venusbecken. 1 St.

274...18 C. Ziczac. Das ziczac Venusbecken. $1 \mathrm{St}$. 275...19. C. Violata. Das blaue Venusbecken. $1 \mathrm{St}_{\text {, }}$ 276...20 C. Nebulosa. Das wolkigte Vcnusbecken. 1 St.

\section{Lade. 23.}

\section{Venus. Die Venusnuschel.}

1. Subovatox. Eyförmige.

277...1 V. Chione. Der braune Sonnenstrahl. Gmel. Venus Chione, sp. 16. Chemn. 6 t. 32 fig. 343. 11 St.

278...2 V. Lusoria. Die Spieldublette. Gm. Venus Chione, sp. 16 ß. Chemn. 6 t. 32 f. 340 . inwendig bernalt und vergoldet. 2 St.

279...3 V. Fustigata. Die gestäupte Venusmuschel. 3 St. 280...3 * V. Triradiata. Die dreystrahlige Venusmuschel. Gm. Venus triradiata, sp. $\$ 5$. Chemn. 6 tab. 34 f. 358. 3 St.

281.... V. Maculata. Die gefleckte Venusmuschel. Gm. Venus maculata, sp. 17. Chemn. 5 t. 33 f. 345.6 St. 282...5 V. Ericyna. Die unvergleichliche Venusmuschel. Gm. Venus Erycina, sp. 15. Chemu. 6 t. 32 f. 337. 5 St. 283...6 V. Spuma. Die unăchte Venusmuschel. Gm. Venus Erycina, sp. 13. Cliemn. 6 t. 32 f. 338. 1 St. 


\section{Venus, Die Venusmuschel.}

\section{Triangulares. Dreyeckige. \\ * Glabrae. Glatte.}

$281 . . .7$ V. Islandica. Die isländische Venusmuschel. Gmel. Venus Islandica, sp. 15. Chemn. 6 t. 32 f. 341. 7 St. 285...8 V. Ferröensis. Die feröeirche Venusmuschel. Gm. Venus Islandica, sp. 15. $2 \mathrm{St}$.

286...9 V. Pitar. Das Eisduplett. Gm. Venus Islandica, sp. 15 \%. Chemn. 6 t. 32 f. 342.2 St.

\section{Lade 24.}

287...10 V. Meretrix. Die leichtfertige Venusmuschel. Gm. Venus Mercenaria, sp. 14. Chemn, 10 t. 171 f: 1659 , 60. 3 St.

$288 \ldots-\alpha-\ldots 2$ St.

289...11 V. Gravida. Die schwangre Venusnaschel. Gmel. Venus opima, sp. 44 . Chemn. 6 t. 34 f. $35 \%$. 4 St. $290 \ldots-a-2 \mathrm{St}$.

$291 \ldots 12$ V. Protracta. Die vorzügliche Venusmuschel. 2 St. $292 \ldots 13$ V. Castrensis. Die Lagervenusmuschel. Gm. Venus - castrensis, sp. 20.

293... - $\alpha$ - Chemn. 6 t. 35 fig. 367, 68. 7 St. $294 \ldots-\beta$ - - - Chemn. 6 t. 35 fig. 330, 81. 5 St. 295...14 V. Fulminea. Die glühende Venusmuschel. Gmel. Venus castrensis, sp. 20. Chemn. 6 t. 35 f. 369, 370. 9 Stïck.

$296 \ldots 15$ V. Undata. Die wellenförmige Venusmuschel. Gnı. Venus castrensis, sp. 20. 3. St.

$297 . . .16$ V. Polita. Die geschliffene Venusmuschel. Gm. Venus castrensis, sp.20. Ghemn. 6 t. 35 f. 371, 72. 2St. 299...17 V. Japonica. Die japanische Venusmuschel. Gmel. Venus Chione, sp. 16 \%. Chemr. 6 t. 99 f. 944 . ist. 299...18 V. Menstruata. Die blutende Venusmuschel. 1 St. 500...19 V. Sulcata. Die gerillie Velizsmuschel. 2 St$301 . .20 \mathrm{~V}$. Tripla. Die dreyechige Veninsmuschel. Gm. Ven: tripla, sp. 29. Chemn. 6 t. 31 f. 382 . S. St. 303...21 V. Mactroides, Die Korbvenusmuschel. Gm. Venus corbicula, sp. 39. Chemn. 6 t. 31 fig. 326. 3 St. 


\section{Lade 25.}

** Sulcatae. Gefurchte.

309...22 V. Dioxe. Die stachligte Wenusmuschel. Gm. Venus Dione, sp. 1. Chemn. 6 t. 27 f. 271, 72, Knorr Verg.1 t. 4 fig. 3 \& 4 . 3 St.

$304 . .23 \mathrm{~V}$. Circinata. Die gereifte Venusmuschel. Gm. Venus guineensis, sp. 10. Chemm. 6 t. 30 frg. 3 ir. $2 \mathrm{St}$. 305...24 V. Porcata. Die gestrahlte Venusmuschel. Gnel. Venus striata, sp. 49 . Cliemn. 6 t. 31 f. 365,65 . I St. 306...25 V. Striata. Die gestreifte Venmsmuchel. Gm. Venus striata, sp. 49. Chemn. 6 t. 34 fig. $355,65.1$ St.

30\%...26 У. Gallina. Die Menne. Gm. Venis gallina, sp. 9, Lister t. $281 \mathrm{fig} .119$ t. $282 \mathrm{fg}$. 130 . Chemn. fo t. 80 308-10. Knom Verg. 5 t. 13 f. 2-5. 2 St.

308...27 V. Paphia. Das alte gerunzelte Weib. Gm. Vênus paphia, sp. 2. Chemn. 6 t."27 fig. 274-76́. "Finorr Verg. 2 t. 29 fig. 2 \& -6 t. 5 fig. 6.9 St.

$309 \ldots-\alpha-1$ St.

310...28 V. Ebriosa. Die Schwelgerin. Gm. Venus Dysera, sp. 4. 1 St.

$311 \ldots-\alpha \div 1$ St.

312...29 V. Dysera. Die breitblättrige Venusmuschel. Gm. Venus dysera, sp.4. Lister t. 277 fig. 114. Chemn. 6 t. 27 fig. 979,80 . Knori Verg. 4 t. $2 \frac{4}{2}$ fig. 3,6 t. 10 fig. 2. 2 St.

313... s0 Lamellata. Die blättrige Venusmuschel. Gn. Venus dysera, sp. 4. Chemn.6 t. 27 fig. 280. 1.St.t. .' 314...31 V. Stricta. Dio weisse platte Venusmuschel. Gm. Venus dysera, sp.4. Chemn. 6 t. 28 fig. 289- 1 St. 315...32 V. Pacifica. Die Friejens-Flagge. Gm: Venus plicata, sp. 30. Chemn. 6 t. 28 fig. 295-9\%. Valentin Abh. t. 15 fig. 21. 1 St.

\$16...33 V. Maria. Die gefleckte Venusmuschel. Gm. Venus. marica, sp. 3. Chemn. 6 t. 27 fig. 282-84: Argent. zocm. t. 12 fig: C. 5 St.

317...34 V. Clathrata. Die gegitterte Venusmuschel. Gmel. Venus dysera, sp. 4. Chemn. 6 t. 28 fig. 290. ' 9 st. $318 . .35 \mathrm{~V}$. Fluminea. Die grüne Venuimuschel aus süssem Wasser. Cliemn. 6. t. so fig. 328. I St. 
319...36 V. Donacina. Die eckige Venusmuschel. Gm. Venus donacina, sp. 142. Knorr Verg. 6 tab. 28 fig.7. 5 St. 320...37 V. Rostrata. Die geschnabelte Venusmuschel. Kein Dublett. Chemn. 10 t. 172 fig. 1670, 71. 1 St.

\section{Donax. Die dreyeckige Muschel.}

321...1 D. Scortum. Die Barth-Venus oder Venus-Prinzess. Gm. Donax scortum, sp. 1. Chemn. 6 t. 25 f. 243248. Knorr Vergn. 6 t. 7 f. 7. 4 St.

322...2 D. Serra. Die gezackte dreyeckige Muschel. Gmel. Donax rugosa, sp. 3. Chemn. 6 tab. 25. fig. 351, 52. 2 Stiick.

323...3 D. Cuneata. Der keilförmige Dreyeck. Gn. Donax Cuneata, sp. 7. Chemn. 6 t. 26 f. 260. 9 St.

s24...- - - s St.

325...4 D. Trunculus. Das Klötzchen. Gm. Donax trunculus. sp. 4. Chemn. 6 t. 26 f. 253, 54. Knorr Vergn. 1 t. 7 f. 7. 11 St.

326...5 D. Denticulata. Das gezähnelte Dreyeck. Gm. Donax denticulata, sp. 6. Ch. 6 t. 26 f. 256, 5\% 6 St.

327...6 D. Violacea. Das violenblaue Dreyech. Gm. Donax denticulata, sp. 6.1 St.

\section{Lade 26.}

\section{Corbula. Das Körbchen.}

328...1 C. Polygona. Das winklige Körbchen. Gm. Tellina multangula, sp. 9. Chemn. 6 t. 9 f. 77. 3 St.

$-\ldots-$ - $-2 \mathrm{st}$.

329...2 C. Anomala. Das unächte Körbchen. Gm. Venus deflorata, sp. 24. Chemn. 6 t. 9 f. 79, 80. 2 St.

\$30...3 C. Rosea. Das rothe Körbchen. Gm. Ven. deflorata, sp. 24. Chemn. 6 t. 9 f. 82. 3 St.

331...4 C. Radiata. Das bunte Körbchen. Gm. Venus deflorata, sp. 24 . Chemn. 6 t. 9 f. 81 . 4 St. Zwey Paar sind geschliffen und polist.

332...5 C. Violacea. Das bläuliche Körbchen. Gmel. Venus deflorata, sp. 24. 2 St.

339...6 C. Porcata. Das gefurchte Körbchen. 1 St. 
334...7 C. Purpurascens. Das purpurfarbene Körbchen. 1 St. 335...8 C. Rugosa. Das gerunzelte Körbchen. 1 St. 336...9 C. Tumida. Das bäuchige Körbchen. Gmel. Chama oblonga, sp. 10. Chemn. 7 t. 50 f. 505. 1 St. 337...10 C. Angulata. Das winklige Körbchen. I St. 358...11 C. Elongata. Das längliche Körbchen. $1 \mathrm{St}$. 339...12 C. Minuta. Das kleine Körbchen. Gm. Solen minutus; sp. 11 . Chemn. 6 t. 6 f. 51, 52. 1 St.

340...13 C. Glans. Die Eichel-Doublette. Gm. Chama calyculata, sp. 7. Chemn. 7. t. 50 f. $500,501.1$ St.

341...14 Eine unbekannte Muschel, dem äussern Anschein eine Miesmuschel, dem Schlosse nach eine Arche. 1 St.

\section{Lade 27.}

\section{Tellina. Die Telline.}

\section{Lanceolatae. Lanzetförmige.}

342...1 T. Listeri. Die Listerche Telline. Gm. Tellina vir* gata, sp. 3. Cliemn, 6 t. 8 f. 73. 2 St.

343...2. T. Virgata. Die gestrahlte Telline. Gm. Tellina virgata, sp. 3. Chemn. 6 t. 8 f. $66-72.6$ St.

344...3 T. Virginea. Die schneeweisse Telline. Gm. Tellina virgata, sp. 3. $3 S_{t}$.

345...4.T.Rosea. Die rosenrothe Telline. Gm. Tellina rostrata, sp. 22. Chemn. 6 t. 10 f. 96. Knorr Vergn, 4 t. 2 f. $3-5.2$ St.

346...5 T. Spengleri. Die Spenglersche Telline. Gm. Tellina Spengleri, sp.' 30. Chemn. 6 t. 10 f. $88-90.2$ St.

$347 \ldots 6 \mathrm{~T}$. Foliacea. Die Goldzunge. Gm. Tellina foliacea, sp. 18. Chemn. 6 t. 10 f. 95.2 St.

348...7 T. Semiplanata. Fothe Tulpenblätter. Gm. Tellina operculata, sp. 22. Chemn. 6 t. 11 f. 97. Knorr 6 t. 12 f. 1: 3 St.

349...8 T. Porcellanea. Die porcellan Telline. Gm. Tellina radiata, sp. 21. 9 St.

350...9 T. Radiata. Die dreystrahlige Telline. Gm. Tellina radiata, sp. 21. Chemn. 6 t. 11 f. 102. Knorr Vergn.

1 t. 19 f. 1-4. t. 2 f. 4 \& t. 2 f. 2,15 St 
351...10 T. Ferröensis. Die Ferröensische Telline. Gm. Tellina feroensis, sp. 31. Lister Conch. tab. 394 f. 241. Chemn. 6 t. 10 f. 91.4 St.

352...11 T. Picta. Die gemalte Telline. Gm. Tellina feroensis, sp. 31. 1 St.

353...12 T. Nebulosa. Die wolkige Telline. Gmel. Tellina feroensis, sp. 31. 4 St.

354...13 T. Gari. Die amethystfarbene Telline. Gm. Tellina gari, sp. 5. Chemn. 6 t. 10 f. 9g. Knorr Vergn. 6 t. 12 f. $2.2 \mathrm{St}$.

355...14 T. Violacea. Die blaue Telline. Gm. Solen diphos, sp. 13 var. 3 St.

356...14 ${ }^{*} \mathrm{~T}$. Rostrata. Die schnabelfürmige Telline. Gmel. Solen diphos, sp. 13. Chemn. 6 t. 7 f. 53, 54. 2 St.

357...15 T. Rubicunda. Die röthliche Telline. Gmel. Solen sanguinolentus, sp. 18. Chemn. 6 t. 7 f. 56. 1 St.

558...16 T. Striatula. Die blasrothe Telline. Gmel. Solen striatus, sp. 19. Chemn. 6 t. 7 f. 57, 58. 2 St.

559..17 T. Coccinea. Die kleine rothe Telline. Gm. Tellina coccinea, sp. 3\%. Chemn. 6 t: 12 f. 109. 5 St.

360...18 T. Complanata. Die platte Telline. Gmel. Tellina hyalina, sp. 33. Chemn. 6 t. 11 f. 99.2 St.

361...19 T. Fragilis. Die dünne Telline. Gm. Tellina fra. gilis, sp.. 6. Chemn. 6 t. 9 f. 84.4 St.

362...20 T. Depressa. Die plattgedrüchte Telline. Gm. Tell. depressa. sp. 55. Gualt. test. t. 88 f. H. J. L. 4 St.

\section{Lade 28.}

363...21 T. Angulosa. Die winklige Telline. Gm. Tellina angulata, sp. 4. Chemir. 6 t. 9. f. $74,75.4$ St.

364...22 T. Ovata. Die eyförmige Telline. Gm. Tellina inflata, sp. 8. Chemn. 6 t. 9 f. 76.1 St.

365...23 T. Angulata. Die echige Telline. 2 St.

\$66...24 T. Laevigata. Die geglättete Telline. Gm. Tellina laevigata, sp. $20 . \quad$ Chemn. 6 t. 12 f. 111 . Schröters Einl. 2 p. 649 t. 7 f. 10.3 St.

$36 \%$..25 T. Solaris. Die Sonnenstrahlen-Telline. $2 \mathrm{St}$.

368...26 T. Lingua felis. Die Katzenzunge. Gm. Tellina lin• gua felis, sp. 2. Chemn. 6 t. 8 f. 65 . Knorr Vergn. 2 t. 2 f. 1. 1 St.

$\$ 69 . . . \alpha-\alpha$ - 1 St.

$370 \ldots-\beta-$ - sebr selten. $1 \mathrm{St}_{\mathrm{c}}$ 


\section{Tellina, Die Telline.}

\section{Rotundatce. Ründliche.}

371...27 T. Triangularis. Die dreiwinklige Telline. Gmel. Tellina trilatera, sp. 28. Chemn. 6 t. 10 f. 85.2 St. 372...28 T. Aniöenissima. Die vortrefliche Telline. GmelTellina Remies, sp. 66. I St.

373...29 T. Remies. Die Sand-Telline. Gm. Tellina Remies, sp. 66. Chemn, 6 t. 12 f. 112, 13. 1 St.

374...30 T. Scobinata. Die Robbenzunge. Gm. Tellina scobinata, sp. 68. Chemn. 6 t. 13 f. 122-24. Knorr 6 t. 37 f. 3. 3 St.

375...31 T. Deavrata. Die wachsgelbe Telline. Gm. Venus pensilvanica, sp. 71. Chemn. 7 t. 37 f. 394. \$ St. $376 \ldots-\alpha-1$ St.

377...32 T. Pensilvanica. Die Pensilvanische oder Krugel. Telline. Gm. Venus pensilvanica, sp. 71. Chemn. 7 t. 37 f. 396. $3 \mathrm{St}$.

378...33 T. Edentula. Die zahnlose Telline. Gmel. Venus edentula, sp. 80 . Chemn. 7 t. 40 f. $427-29.2$ St.

\section{Lade 29.}

\section{Corculum. Die Herzmiuschel.}

979... I C. Cardissa. Das mit Blut besprengte Herz. Gmel. Cardium cardissa, sp. 2. Chemn. 6 täb. 14 f. 143, 44 . 1 Stïck.

$380 . . . \infty-\infty \mathrm{St}$.

$381 \ldots-\beta-1$ St.

$382 . . . \mathrm{Tr}-1$ St.

385...2 C. Humanum. Das Menschenherz. Gm. Cardium cardissa, sp. 2. Chemn. 6 t. 14 f. 145,46 . 1 St. 284...3 C. Inflatum. Das bäuchigte Herz. Gmel. Cardium monstrosum, sp. 29. Favanne, PI. 51 f. E. 1. Z St. 385...4 C. Dolorosum. Das schwermüthige Herz. Gm. Cardium monstrosun, sp. 29. Chemn. 6 tab. 14 fig. 149, 150. 2 St. 


\section{Fragum. Die Erdbeere.}

386... 1 F. Hemicardium. Das dreiseitige Herz. Gm. Cardium hemicardium, sp. 5. Chemn. 6 t. 16 f. 159-61. 3 St. $38 \% . .2$ F. Flavum. Die gelbe Erdbeere. Gm. Card. fragum, sp. 13. Chemn. 6 t. 16 f. $165,67.2$ St.

388...3 F. Unedo. Die rothe Erdbeere. Gm. Cardium unedo, sp. 14. Chemn. 6 t. 16 f. 168, 69. Knorr Vergn. 2 t. 29 f. 2. 6 St.

389...4 F. Medium. Die reife Erdbeere. Gm. Cardium medium, sp. 6. Chemn. 6 t. 16 f. 162-65. Finor Verg. 1 t. 29 f. 5,6 . t. 20 f. 5 . \& St.

\section{Lade 30.}

\section{Cardium. Die herzförmige Muschel.}

\section{Lamellatae. Blättrige.}

390...I ! C. Coștatum. Das hochgerippte Herz. Gm. Cardium costatum, sp. 1. Listei 1 t. 327 f. 164. Knorr Vergn. 1 t. 28 f. \&. Chemn. 6 t. 15 f. 151, 52. 2 St.

\section{Cardium. Die herzförmige Muschel. \\ 2. Costatce. Gerippte.}

$391 . . .2$ C. Leucostomum. Das gelbmündige Herz, oder Feitener Dublette. Gm, Cärdium magnum, sp. 16. Lister Conch. t. 331 f. 168 ß. Chemn. 6 t. 17 f. 179. 2 St. 392...3 C. Aurantiacum. Das orange Herz. 1 St.

393... 4 C. Cerratum. Das gelbe Herz. Gm. Cardium laeviga. tum, sp. 18. Chemn. 6 t. 18 f. 189. 3 St. $394 . . .5$ C. Fragile. Das dünnschalige Herz. 2 St. $395 . .6$ C C. Oblongum. Das länğliche Herz. Gmel. Cardium oblongum, sp. 34. Chemin. 6 t. 19 f. 1So. 3 St.

\$96...7 C. Arca, Das archenformige Herz. 1. St.

$397 . .8$ C. Groenlandicum. Das Grönländische Herz. Gmel. Cardium grönlandicum, sp. 22. Chemn. 6 t. 19 f. 198. I Stïck,

598...9 C. Ringens. Das Sperrmaul. Gm. Cardium ringens, sp. 31. Chemn. 6 t. 16 f. 170 . Knorr Vergn. 4 t. 14 f. 3 \& 6 t. 3 f. 4. 1 St. 
399...10 C. Myoideum. Das Miesmuschelähnliche Herz. 1 St. 400...11 C. Latum. Die breite herzförmige Muschel. Gmel. Cardium latum, sp. 36. Chemn. 6 t. 19 f. 192, 193. 1 Stück.

401...12 C. Aeolicum. Das Ost-und West-Herz. Gm. Cardium aeolicum. Chemn. 6 t. 18 f. $187,88.1$ St.

402. $\therefore 15$ C. Tuberculatum. Die Carthaginensische Herzmuschel. Gm. Cardium tuberculatum, sp. 11. Chemn. 6, t. 17 f. 173. Knorr Vergn. 5 t. 30 f. 2 \& 6 t. 8 f. 3. $2 S t$. 403... 14 C. Ciliatum. Die Isländische Herzmuschel. Gmel. Cardinm ciliatum, sp. 10 . Chemn. 6 t. 19 f. 195, 96. 2 St. verschiedene.

\section{Cardium. Die herzförmige Muschel. \\ 3. Imbricatce. Gedeckte.}

404...15 C. Isocardia. Das gleichseitige Zigel-Herz. Gmel. Cardium isocardia, sp. 12. Lister tab. 323 fig. 160. Chemu. 6 t. 17 f. $174-76$. 2 St.

405...16 C. Campechiense. Die gelbliche Sägeribbe. Gm. Cardium muricatum, sp. 15.4 St.

$406 \ldots-\alpha-$ - Knorr 4 t. 14 f. 5. Chemn. 6 tab. 17 fig. 178. 1 St.

407...17 C. Flavum. Das citronengelbe Herz. Gm. Cardium flavum, sp. 17. Schröters Einl. in die Conch. 2 t. 7 f. 11 a. b. Bonam, recte. 2 f. 72.2 St. 408...18 C. Celatrm. Das gefurchte Herz. 1 St.

\section{Lade 31 .}

\section{Cardium. Die herzförmige Muschel.} 4. Dentatce. Gezähnte.

409...19 C. Muricatum. Das stachlige Herz. Gmel. Cardium aculeatum, sp. $\%$. Lister t. 321 f. 158 . Chemn. 6 t. 15 f. 155-57. Knorr V. 6 t. 3 f. 1. 8 St. $410 \ldots-\alpha-\ldots$ St.

$411 . .20$ C. Echinatum. Das dornige Herz, die Egge. Gm. Cardium echinatum, sp. 8.: Chemn. 6 t. 15 f. 156 , $57,58,59.9 \mathrm{St}$.

$412 \ldots-\alpha-2 \mathrm{St}$. 


\section{Cardium. Die herzförmige Muschel.}

5. Glabrce. Glatte.

413...21 C. Cor auritum. Die grosse Narrenkappe. Gmel. Chama cor, sp. 1. Lister Conch. t. 275 f. 111. Knorr Vergn. 6' t. 8 f. 1. Chemn. 7 t. 48 f. 489.4 St. 414...22 C. Corculum. Die kleine N'arrenkappe. 1 St.

\section{Lade 32.}

\section{Anomia. Die Terebratel.}

15...1 A. Striata. Die gestreifte Terebratel. Gm. Anomia dorsata, sp. 40. Chemn. 8 t. 78 f. 710, 711. Naturf. 3 t. 3 f. 1-3. $3 \mathrm{St}$.

416...2 A. Bonae Spei. Die Capsche Terebratel. Gm. Anomia capensis, sp. 35. Chemn. 8 t. 77 -f. 703 a. b. c. 1 St.

417...3 A. Truncata. Die kleine abgestumpfte Terebratel. Gm. Anomia truncata, sp. 14. Chemn. 8 t. 77 f. 701 a. b. 5 St.

718...4 A. Caput serpentis. Der Schlangenkopf. Gm. Anomia Caput serpentis, sp. 21. Chemn. 8 t. 78 f. 712. 8 St.

419...5 A. Vitrea. Die durchsichtige Terebratel. Gm. Anomia vitrea, sp. 38. Chemn. 8 t. 78 f. 707, 8. Knorr Verg. 4 t. 30 f. 4. 2 St.

420...6 A. Coleoptrata. Die geflügelte Terebratel. $2 \mathrm{St}$.

\section{Fcnestella. Die Fensterluke.}

421...1 F. Ephippium. Die sattelförmige Fensterluke. Gmel. Anomia Ephippium, sp. 3. Chemn. 8 t. 76 f. 692, 93. Lister t. 204 f. 38.11 St.

422...- $\alpha-6$ St.

$423 \ldots-\beta-\beta-1 \mathrm{St}$.

$424 \ldots-\gamma-2$ St.

425...2 F. Cepa. Die Zwiebel. Gmel. Anomia Cepa, sp. 4. Chemn. 8 t. 76 f. 694,695 . Knorr Vergn. 6 t. 9 f. 5 . 2 Stück.

$426 \ldots-\infty-\infty$ - 1 St.

127...3 F. Electrica. Die Bernstein Fensterluke. Gm. Anomia electrica, sp. 5. Chenun. \& t. 76 f. 691. Knorr Vergn. 5 t. $25 \mathrm{f} .6 .1$ St. 
428...4 F. Sq amula. Die schuppenförmige Fensterluke. Gm. Anomia squamula, sp. 6. Chemn. 8 t. 77 f. 697. 1 St. 428a..4a Unbekannte. 4 St.

\section{Lade 33.}

\section{Spondylus. Die Lazarusklappe.}

429...1 S. Dominicensis. Die weisse Lazarusklappe mit hellrothen Wirbel. Gm. Spondylus Gaedaropus, sp. $1 \delta$. Chemn. 7 t. 45 f. 465 . Knorr. Vergn. 5 t. 9 f. 1 \& 6 t. 9 f. 1. 5 St.

$430 \ldots$ - $\approx-2$ St.

431...2 S. Rufus. Die braunrothe Lazarusklappe. Gm. Spondylus Gaedaropus, sp. 1. 2 St.

432...3 S. Regius. Die königliche Lazarusklappe. Gm. Spondylus Gaedaropus, sp. 1. Chemn. 7 t. 46 f. $4 \pi 1$. 3 St. $483 . . .-\infty-1$ St.

\section{Lade 34.}

434...4 S. Gaedaropus. Die gezachte Klappnuschel. Gmel. Spondylus Gaedaropus, sp. 1. Chemn. 7 t. 44 f. 459. Knorr Vergn. 1 t. 7 f. 1.6 St.

\$35...5 S. Marisrubri. Die Lazarusklappe aus dem rothen Meere. Gmel. Spondylus Gaedaropus, sp. $1 \delta$. Ghem. 7 t. 44. Mart. f. 460 . 4 St.

436...6 S. Labrum. Die Badewanne. Gm. Spondylus Gaedaropus, sp. 1. 1 St.

437...7 S. Cruentatus. Die blutrothe Lazarusklappe. Gmel. Spondylus Gaedaropus, sp. 1. 2 St。

\section{Lade 35.}

438...8 S. Macis. Die Muscatblume. Gm. Spondylus Gaeda. ropus, sp. 1. $1 \mathrm{St}$.

439...9 S. Ducalis. Die breitblättrige Lazarusklappe. Gmel. Spondylus Gaedaropus, sp. 1 \&. Chemn. 7 t. 47 f. 476. 477. 3 St.

440...10 S. Variegatus. Die bunte Lazarushlappe. Gm. Spon= dylus Gaedaropus, sp. 1 \%. Chemn. 7 t. 45 f. 464 . 1 St. 441...11 S. Pes-asininus. Der Eselstuss. Gmel. Spondylus Gaedaropus, sp. 1. Chemn, 7 t, 46 f $472,72,1 \mathrm{St}^{*}$ 
442...12 S. Hystrix. Der Igel. Gm. Spondylus Gaedaropus, sp. 1 n.' Chemn. 7 t. 45 f. 470.2 St.

443...13 S. Aurantiacus. Die orange Lazarnsklappe. Gmel.

Spondylus Gaedaropus, sp. 1 \&. Chemn. 7 t. 45 f. 463.

2 St. und ein Untertheil.

- ...- $\alpha$ - E Ein grosses Exemplar.

444...14 S. Pictorum. Die hochrothe Lazarusklappe, eine vortreffliche Oberschaale allein.

445...15 S. Plicatus. Die gefaltete Lazarusklappe. 3 St.

$445 \ldots 16$ S. Cristatus. D́ie kammförmige Lazarusklappe. 1 St.

Lade 36.

\section{Chama. Die Chama-Muschel.}

447...1 C. Lazarus. Die Muscatblüthe. Gm. Chama Lazarus, sp. 11. Knorr Vergn. 1 t. 8 f. 1 . Chcmn. 7 t. 51 f. $50 \%$, 8. 2 St.

448...2 C. Cryphoides. Die geschnörkelte Felscnmuschel. Gm. Chama gryphoides, sp. 12. Chemn. 7 tab. 51 fig. 510, 519. Knorr. Vergn. 6 t. 16 f. 1 . 7. St.

449...3 C. Macerophylla. Die gelbe Chama. Gmel. Chama macerophylla, sp. 17. Chemn. 7 t. 52 fig. 514-51\%. Knorr Vergn. 1 t. 21 f. 2 \& 5 t. 24 f. 1. 2 St.

450...4 C. Lamellata. Die blättrige Chama. Gm. Chama bicornis, sp. 13. Chemn. 7 t. 52 f. 519.1 St.

451...5 C. Muricata. Die stachlichte Chama. Gm. Chama bicornis, sp. 13. Chemn. 7 t. 52 f. 518 . ist.

452...6 C. Fossilis. Die Chama vor Courtignon. 1 St. 455...7 C. Gryphites, 3 St.

\section{Multivalves. Vielschaaligte.}

$$
\text { Lațe } 37 .
$$

Chiton. Die Käfermuschel.

454...1 C. Brunneus. Die braune Käfermuschel. Gm. Chiton magellanicus, sp. 12. Chemn. 8 t. 95 f. 797. 2 St. 
455...3 C. Squamosus. Die schuppenvolle Käfermuschel. Gm. Chiton squamosus, sp. 5 . Chemn. 8 t. 94 f. $788-91$. Spengler Bescbreib. berl. Naturf. 1 p. 242 t. 7 f. L. M. 7 Stück.

456...4 C. Variegatus. Die bunte Käfermuschel. Gm. Chiton marmoratus, sp. 15. Chemn. 8 t. 95 f. 805.10 St.

45\%...5 C. Carners. Die hornfarbige Käfermuschel. 2 St.

458...6 C. Striatus. Die gestreifte Käfermusshel. I St-

459...7 C. Griseo-Rubicundus. Die graurüthliche Käfermu: schel 1 St.

460...8 C. Viridis. Die grŭ̀ne Käfermuschel. $2 \mathrm{St}$.

461..9 C. Magellanicus. Die magellanische Käfermuschel.

Gm. Chiton Magellanicus, sp. 12. Chemn. 8 tab. 95

fig. 797, 98. $6 \mathrm{St}$.

452...10 C. Granulosus. Die branie granulirte Käfermuschel.

Gm. Chiton piceus, sp. 17. Chemn. 8 t. 95 f. 807-10.

Chem. Chit, t. 2 f. $6 \& 6$ a. b. c. 6 St.

163...11 C. Atro-Irididis. Die dunkelgrüne Küfermuschel. I St.

\section{Lade 38.}

\section{Lepas. Die Seetulpe.}

464... I I. Diadema. Die Wallfischpocke. Gm. Lepas Diadema, sp. 4. Lister Conch. t. 445 f. 288. Born Mus. Caes. test. t. 1 f. $5,6 \&$ p. 7 f. a: Chemn. 8 tab. 99 fig. 843, 44. 1 St.

465...2 L. Tintinutbulum. Die grosse Seetulpe. Gm. Lepas Tintinnabulnm, sp. 3. Lister t. 443 f. 285. Knor V. 2 t. 2 f. $68=5$ t. 80 f. 1 . Chemn. 8 t. 97 f. $8 \geq 8-31$. 10 Stïckle

465...3 L. Rosata. Die rosenrothe Seetulpe. Gm. Lepas Tintinnabulum, $\beta$. Tulpia alba. Ch. 8 t. 97 f. 832. 1 St. 467...4 L. Senticosa. Die mit Spitzen besetzte Seetulpe. 1 St. 468...5 L. Spinosa. Die dornigte Seetulpe. Gm. I.epas spinosa, sp. 22. Schr. berl. Naturf. 5 tab. 5 fig, 3, 4. Chemr. Chemn. Conch. 8 t. 98 f. 840 \& t. 99 f. 841 . 2 St.

469...6 L. Echinata. Die stachlichte Seetulpe- Gmel. Lepas spinosa, sp. 22. Chemn. 8 t. 98 f. 840.6 St.

470...7 I. Ovata. Die eyrunde Setulpe. $3 \mathrm{St}$.

471...8 L. Testudinaria. Die Schildkröten-Seetulpe. Gm. L. testudinaria, sp. 6. Knorr V. 3 t. 30 f. 3, 4. 1 St. 


\section{Bernicla. Die Entenmuschel.}

172...1 B. Anatifera. Die weisse Entenmuschel. Gm. Lepas anatifera, sp. 13. Knorr Vergn. 2 t. 30 f. 4, 5. Chem. 8 t. 100 f. $853-55.12 \mathrm{St}$.

$473 \ldots-\infty-\rightarrow$ Gm. Lepas anatifera, sp. 12. Lister t. 440 f. 283. Lister exerc. anat. t. 19 f. 4, 5. Chemn. 8 tab. 100 f. 856. 2 St.

474...1* B. Striatula. Die gestreifte Entenmuschel. $3 \mathrm{St}$. 475...2 B. Inflata. Die bäuchige Entenmuschel. 2 St.

476...3 B. Pollicipes. Die Fusszehe. Gm. Lepas pollicipes, sp. 24. Knorr Vergn. 5 t. 13 f. 7 " Chemn. 8 t. 100 f. $851,52.4$ St.

477...4 B. Mitella. Das Mützgen. Gm. Lepas Mitella, sp. 10. Chemn. 8 t. 100 f. $849,50,2$ St.

\section{Pholas. Die Pholade.}

478... I P. Costatus. Die gerippte Pholade. Gm. Pholas costata, sp. 2. Knorr 2 t. 25 f. 4. Chemn. 8 tab. 101 f. 863.1 St.

479...2 P. Dactylus. Die schmale Pholade. Gm. Pholas Dactylus, sp. 1: Chemn. 8 t. 101 f. 869.1 St.

\$30...3 P. Orientalis. Die orientalische Pholade. Gm. Pholas orientalis, sp. 7. Lister t. 431 f. 274 . Chemn. 8 t. 101 f. 860 . Walch. Naturf. 13 p. 86 t. 3 f. 15.1 St.

481...4 P. Crispatus. Die Lockenpholade. Gm. Pholas crispata, sp. 6. Lister t. 456 f. 279. Chemn. 8 t. 102 f. 872, 73. 1 St.

482...5 P. Pusillus. Die kleine Pholade. Gm. Pholas pusilla, sp. 5. Chemn. 8 t. 102 f. 867-71. 5 St. davon 2 St. in Holz gebohret.

483...6 Zwei Steine mit Pholaden.

484...7 Zwei dito mit Lockenpholaden.

A. Eine Schachtel mit 30 St. div. unreifen \& monstrüsen Perlen, B. Eine dito mit 45 dergleichen.

C. Eine dito mit 12 dergleichen.

D. Eine dito mit 4 durchgeschnittenen.

E. Eine dito mit Moscowitischen Perlen.

T. Eine dito mit kleinen Loth-perlen.

G. Kallistücke, in denen Perlen befindlich.

H. Eine Dose mit geschliffenen u. ungeschliffenen Pfauenstein。 


\section{Zweite Abtheilung.}

\section{$M i n$ e r a $l i$ i $n$.}

1. 3 Stïck Circon Geschiebe.

2. 4 St. desgleichen als Ringsteine geschliffen.

3. 4 St. Hyacinthe dito.

4. 3 St. Chrysobarill-Geschiebe.

5. 6 St. Fubin-Spinell, christallisirt.

6. Dito Körner und dito Sand aus Ceylon.

7. 3 hltine gefasste Rubine und 2 ungefasste Rubin balais.

8. 2 Saphire, einer geschnitten als Pettschaft, ein Geschiebe,

9. 2 Bruchstücke vom Smaragd.

10. 1 Barill als Ringstein.

11. 2 Ostindische edle Granaten, oval geschliffen.

12. Melrrere kleine desgleichen, ungeschliffen.

13. Dito christallisirte Böhn. Granaten, theils durchgebohrt,

14. Dito kleine diverse.

15. Verschiedene christallisirte Granaten.

16. Desgleichen.

17. 6 christallisirte rohe Brasilianische Topase.

18. 2 Dito (Sächsische) Schneckensteine,

19. 1 Dito in der Bergart. (Sachsen.)

20. 1 angeschliffener Brasil. Turmalingrün,

21. 1 gelb gefärbter Berg=Chrystall mit Facetten im Geschmack des Regenten (Diamanten) sehr schön geschliffer.

22. 2 rohe gefürbte Quartze und 6 diverse geschliffene Berg. Christalle, worunter 1 Rauchtopas und 1 Milchquartz.

23. 2 St. Kuggeln von Berg. Cristall geschliffen.

24. 1 Schachtel mit $40 \mathrm{St}$. Türkisen.

25. 1 roher und 3 geschliffene kleine Lapis Iazulli,

26. 1 Schachtel mit div. Flüssen.

27. 1 christallisirter Schörl aus Grönland.

23. I gemeiner strahligter Schörr.

29. Einige Bruchstücke desgleichen.

30. 2 St. Granat in Quarz, angeschliffen.

31. I St. dito in Glimmerschiefer dito.

32. I schöner Berg-Christall aus der Schweiz. 
39. 2 schöne Berg-Christalle, einer vom Gotthardt.

34. 1 schöner Berg-Christall, geschliffen, inwendig mit Strahlstein gerïllt.

35. 1 desgleichen von Grönland, mit Eisen-Glanz überzogen.

36. 2 dito Drusen aus Ungarn.

37. 3 dito Gruppen.

38. 1 dito mit Ziınstein, (extra schön von Zwönitz.)

39. 1 dito mit eingewachsenen Schorl, v. Orenburg (schön.)

40. 1 dito Gruppe, Rauchgrau, (schön.)

41. Berg. Chirystall mit kirschfarbiger: Oberfläche.

42. 1 Schachtel mit verschiedenen Berg-Christallen."

43. 2 Si. Amethyst-Quarz.

44. 2 St. Desgleichen.

45. 1 Echachtel desgleichen.

46. 2 S.. desgleichen.

47. 2 St. desgleichen.

45. $1 \mathrm{St}$, desgleichen mit Kalzedon überzogen.

49. 1 St. desgleichen angeschliffen.

50. 1 desgleichen Höhle.

51. 1 Dito.

52. 2 Amethist-Quarze.

53. 26 kleine Amethiste, als Ringsteine geschliffen.

54. 5 desgleichen.

55. 1 rauchgrauer Berg-Christall.

56. Rauchgraner Quarz auf Agath (schön.)

57. Desgleicheu auf rothem Eisenstein.

58. 13 einzelne Christallen mit doppelter Pyramide.

59. 2 derbe Stücke Pauchquarz.

60. 2 Quarz-Drusen.

61. 2 desgleichen.

62. 3 desgleichen.

63. A desgleichen.

64. Gehacktex Quarz mit Bleiglanz.

65. 1 Schachtel diverse.

66. 1 Quarz-Druse mit Bleiglanz.

67. 2 Quarz-Drusen.

68. 1 Quarz-Druse mit kirschfarbiger Oberfäche.

69. 2 Quarz-Drusen.

70. 3 desgleichen.

71. 3 desgleichen.

72. I desgleichen. 
73. 1 Quarz-Druse auf Trapp von Island.

74. 2 Quarz-Drisen von Island.

75. 1 Schachtel mit versch. Quarz-Drusen,

76. 1 desgleichen.

77. At Stiick kirschrothe Hyacinth da Compostell.

78. I St. Kieselschiefer.

79. 7 St. verschiedene Feuersteine, einer mit eingewachsener Terrobratula.

80. 1 Tropf-Kalzedon von Ferröe.

81. 1 dito.

82. 1 dito.

83. 2 Stück dito.

84. 2 St. dito ron Island.

85. 1 St. dito von dito.

86. I St. dito von dito.

87. 1 desgleichen mit Amethyst-Quarz von Zweibrücken.

88. 1 Band-Kalzedon von Ferröe.

89. 1 desgleichen.

90. 1 desgleichen.

91. 2 desgleichen.

92. 3 desgleichen.

93. 2 Stück'Kelzedon。

94. 5 St. desgleichen.

95. 1 Schachtel verschiedener desgleichen;

96. 1 dito Kalzedon-Geschiebe.

97. 2 angeschliffene Band-Kalzedone.

98. 2 dito dito.

99. 3 dito dito.

100. I Schachtel verschiedener geschliffener Carniole, worunter 3 Ringe.

101. 1 roher Carniol (gross.)

102. 2 Stiuck Onix, einer flach oval, der zweite als Vogel kopf brav geschliffen.

103. I Katzen - Augen, oval convex geschliffen.

104. 1 Schubfach mit 135 in verschiedener Form geschliffenen Agathen verschiedener Arten.

105. 1 grosser angeschliffener Band-Agath, ein desgleichen von hummersdorff.

106. 1 desgleichen Augen - oder Ring - Agath mit AmethistQuarz.

107. 1 Agath: 
108. 1 Tropf-Agath.

109. 8 verschiedene Mocca-Steine.

110. 8 desgleichen.

111. 11 Stïck Holzstein (versteinert Holz.)

112. 2 St. desgleichen, gross und schön.

113. I Schachtel diverse desgleichen.

114. 2 Stück Holzstein, schön.

115. 1 St. dito angeschliffen, schön.

116. 2 St. dito dito dito.

11\%. 2 St. dito dito dito.

113. 1 grosse quer geschnittene polirte Platte desgleichen, sehr șchön.

119. 2 angeschliffene dito.

120. 2 Stück dito.

121. I St. grünes dito von Koburg, (sehr selten.)

122. 4. Stück diverses dito.

123. I St. Staar - Stein, schön.

124. I sehr grosser Block versteinert Holz.

125. 8 verschiedene Porphyr-Arten und 1 Stück Brezia. (Puddingstein.)

126. 2 Stück Obsidian von Island.

127. I kleine Edel-Opale, geschliffen.

128. 2 rohe dito.

129. 2 gemeine Opale.

130. 1 Schachtel mit verschiedenen Hydrophan (Weltaugen.)

131. 1 gemeiner Opal.

139. 2 Stüch Kascholong.

133. 5 St. geschliffene Chrisoprase.

154. 2 St. rother Jaspis (1 gross.)

135. 1 St. dito worauf ein Phöbus mit dem Sonnenwagen geschnitten.

136. 3 Messexhefte von Jaspis.

137. 1 Schachtel mit versch. kleinen Jaspis, geschliffen.

158. 1 desgleichen mit desgleichen.

139. 1 buntfarbiger grüner Jaspis Brocat-Stein oder Brocatello antico.

140. 2 St. Egyptischer Jaspis (Kièsel, Mannióc) braun \& gelb.

141. 1 St. Prehnit vom Cap.

142. 1 Schaclatel mit Zoolit und Mandelstein.

143. 1 Zoolit von Island.

144. 1 desgleichen von dito. 
145. 1 Zoolit.

146. 1 desgleichen mit Kalkspath vom Harz.

147. 2 dito auf Quarz ron Island.

148. 1 dito strahligt, von Island, schön.

149. 2 dito dito dito.

150. 3 dito dito dito.

151. 2 dito dito dito.

152. 3 dito dito dito.

153. 4 dito von Island.

154. 2 Kreuzsteine vom Andreasberg am Harz (schön.)

155. I Stück Adular (schön).

156. 3 St. ovalconvex geschliffene (Adular) Mondsteine.

157. I Schïssel von Labrador aus Stavern.

158. 2 St. Hormblende.

159. I Schachtel mit Speckstein.

159a. 2 Platten desgleichen.

160. 11 verschiedene angeschliffene Serpentinsteine.

161. 1 Schubfach mit verschiedenen Lava-Stücken.

162. I Schachtel mit Asbest-Harigt, von Cypern.

163. 2 Stück verhärteter do. aus Zöplitz.

164. I St. Bergkork, aus Schweden.

165. 1 St. Bergpapier mit Schieferspath.

165. 1 sehr grosses Stück Kalkspath (Doppelt-Spath) aus Island.

167. 1 desgleichen.

168. I Schachtel verschiedener do.

169. I Stück violeter Kalkspath, von Andreasberg.

170. 5 St. Kalkspath.

171. 1 St. christallisirter Thalkspath (Druse) mit Bleyglana (Andreasberg).

172. 1 St. do. do, von Andreasberg.

173. 2 st. do. do.

174. 1 St. do. do. blättrig, aus Carlsfeld.

175. 4 verschiedene desgleichen.

176. 2 Stück strahligter Kalkstein.

177. 1 St. Stinkstein.

178. 1 St. sogenannter christallisirter Sandstein, (Kalk) vo Montmartre.

179. 1 grosses Stück do.

180. I Schachtel kleiner Kalkspath-Drusem.

I81. $2 \mathrm{Kalkspath-Druser,}$

182. 1 do. do. 
183. 2 Kalkspath-Drusen.

184. 4 desgleichen.

185. 1 dergleichen.

185. 1 Schachtel verschiedenener hleiner desgleichen。

187. 1 Kalkspath-Druse.

188. I desgleichen.

189. 2 desgleichen.

190. 2 desgleichen.

191. 2 desgleichen.

192. 2 desgleichen.

19.3. I Venus Islandica mit Kalkspath schön ausgefüllt:

194. 2 dergleichen.

195. 2 Kalkspath-Drusen.

196. 3 dergleichen.

197. 1 Schubfach mit 20 verschiedenen Marmor-Platten. aus Spanien.

198. 1 dergleichen mit 18 verschiedenen dergleichen.

199. 1 deigleichen mit 20 dergleichen, aus Spanien.

200. I dergleichen mit 20 dergleichen, daher.

201. 1 dergleichen mit 20 Marmor-, Alabaster- und PorphirPlatten, daher.

202. 1 dergleichen mit 20 Marmor-Platten, daher.

203. 1 dergleichen mit 30 dito dito.

204. 1 Kugel ron Marmor.

205. Eisenblüthe, mehrere Stäbe als Grotte zusammengesezt.

206. I Schriblade mit Kalkzinder und Kalktuff.

207. I Schubfach mit verschiedenen dendritischen Mergel. schiefein.

208. 5 Platten Fisch- und Krebs-Abdrücke in Mergelschiefer, sehr schön.

209. 1 Schubfach mit dito.

210. 1 dito.

211. 1 Schubfach Bytouminöser Meigelschiefer, mit Fisch. und Blätter-Abdrücken.

212. 1 dito mit Fisch-Abdrücken.

213. 1 grosse gelbe Flusspath-Druse, von Gersstorf.

214. 1 Stïck grïner Flus:path, von Wildenfels.

215. 2 St. blauer dito vom Harz und Freiburg.

216. 1 St. grüner dito mit Quarz, von Joachimsthal.

217. 1 St. grïnlichter Flisspath überzogen mit Quarz.

218. 1 St. violetter dito, aus Freyberg. 
219. 1 St, vinletter Flusspath, aus Freyberg.

220. 2 St, gelber dito, aus Freyburg und dem Marienburgischen.

221. 2 St. gelb und violetter dito,

222. I St. Flufsspath, von Freyburg.

223. 3 St. dito.

224. 1 Schachtel verschiedener ảito.

225. 1 dito mit Gips- (Zelenith) Spath..

225. 1 dito desgleichen.

227. 1 Gips-(Zelenith) Druse.

228. 2 St. dito.

229. 1 Schachtel mit Verschiedenem.

230. 2 Stück fasrigter Gips.

2SOa. 1 grolses Stück Gips.

2:1. 2 Stück Schwerspath- (Baryt) Drusens

23\%. 2 St. dito dito.

233. 2 St. dito dito:

234. 1 kettenformig in einander geflochtene dito, fon Wildenmann am Harz.

235. I Stüch vulhanischer Schwrefel.

236. 3 St. geảiegener dito.

237. I St. bytrminöses Holz, von Devonshire.

238. I Schachtel mit Braunkohlen.

2:9. 2 Stïcli Graphit, aus England.

240. 1 St. Kohlenblende.

2i1. 2 Stüch Bernstein, dunkel und ziemlich gross。

242. 4 St. dito dito etwas kleiner:

243. 5 St. dito dito.

244. 12 St. Aito dito.

245. S St. dito dito.

245. 1 Schachtel hleiner dito.

24\%. 1 Stïck geschliffen Bastard dito, gelb und weiss.

248. 1 Schachtel mit verschiedenen Perlen von dito.

249. I Stüch Bernstein mit einem Frosch, schön.

250. 6 St. mit Fliegen, Kürfern nnd dergl., schön."

251. 9 dito mit dergleichen.

252. 9 dito dito.

253. 4 St. dito, 2 mit Spinnen und \& mit Ameisen, schon.

254. A Si, dito nit dergleichen, schön。 


\section{Metalle.}

255. 1 hlein Pack mit Platina de Pinto.

256. 1 schöne grosse Stuffe gediegen Gold in Quarz, aus Mexico.

257. 1 Stuffe blättriges gediegen Gold, sehr reich auf Quarz, von Schemmitz.

258. 1 dito Quarz-Druse mit igediegen Drath-Gold, aus Ungarn.

259. 1 dito gediegen Gold in Quarz mit Schwefelkies, aus Adelsfort in Schireden.

260. 1 dito gewachsen Gold mit Kupferglaserz, Yupfergrün and Quarz, vom Aardalischen Kupferwerk in Bergenhuslehn in Norwegen.

261. Gediegen Gold in Quarz mit Schwefelkies, von Aastlewold in England.

262. Desgleichen. (Fundort unbekannt.)

263. Gediegen Gold in Quarze

264. Gediegen Gold in Quarz, aus Schlangenberg in Siberien.

265. Gediegen Gold in Eisenstein, von dem Schlangenberge in Siberien.

266. Gediegen Gold in Quarz, aus Smoland.

267. Gcdiegen Gold in Quarz mit Schwefelkies, von Aetz. wold in Norwegen.

268. 6 verschiedene hleine Goldstufen.

269. 1 Korn gediegen Gold.

270. Gediegen Gold in Schweerspath.

271. 1 Schachtel Goldsand, aus dem Reusfluss im Berner Gebiet.

272. I Glas mit etwas Goldsand.

273. I dito mit dito, aus Thüringen.

274. 1 sehr reiche Stuffe gediegen Silber, vom Himmelsfürsten zu Freiberg, sehr schön.

275. Kalkspath mit gediegen Silber, angeschliffen, von Andreasberg.

276. Kalkspath mit Drathsilber, schön, ron Kongsberg in Norwegen.

277. Dito mit dito, daher, sehr schön.

278. Drathsilber mit violettem Kalkspath, von Freiberg.

279. Drathsilber mit Glaserz und Kupfergrïn. 
280. Haar - und Drathsilber mit Schweerspath, von St. Jacob zu Schneeberg, sehr schön.

281. Gediegen Haar - und Drathsilber in Kallispath, vor Kongsberg.

282. Gediegen Silber in Kalkspath, von Kongsberg.

283. Zelligte Quarz-Druse mit gediegen Silber, schön.

284. Gediegen Silber in Quarz, aus dem Kolivanischen Berg* werk in Siberien.

285. Gediegen Silber in Quarz, von Schneeberg.

286. Gediegen Silber in Kalkspath.

287. Dendritisch gediegen Silber in Quarz, von Johannegeorgenstadt.

288. Gediegen Silber auf Steinmark.

289. Gediegen Silber auf Eisenstein, aus Syberien.

290. 2 Stïck gediegen Silber.

291. 2 St. Fahl-Erz mit gediegen Silber.

292. 5 Kömer Kapellen-Silber.

293. I Stück Glas-Erz auf Kalkspath.

294. 1 St. dendritisch Glas-Erz, angeschliffen, von Donat hinter Erbisdorf.

295. I St. Glas-Erz auf Quarz。

296. 2 St. dito.

297. 2 St. dito.

298. 1 St. christallisirt Rothgülden:

299. 1 St dito dito.

300. $2 \mathrm{St}$, dito dito.

301. 2 St. dito dito.

302. 2 St. dito dito.

303. 3 St. dito dito.

504. 1 Schachtel mit kleinen Stücken,

305. 1 dito dito.

305. 1 St. gediegen Quecksilber, von Hirschfeld.

30\%. 1 St. Quecksilber-Amalgama, von MIuschellandsberg.

308. 1 desgleichen.

309. Christallisirter Zinober.

310. Hellrother Zinober mit Scliwefelkies.

311. Christallisirter Zinober.

312. I Schachtel mit 2 Zinoberstufen.

313. 1 Zinober'stuffe.

314. 2 St. dito.

315. 3 St. dito. 
316. \& St. dito.

317. 2 St. dito.

318. 1 Siück gediegen Kupfer, schön.,

319. I'St. gediegen Kupfer mit Zoolit, von Ferroe.

320. 1 St. dito.

321. I St. roth Kupfer . Erz mit gediegen Kupfer, sclzön,

322. 1 St. gediegen Kupfer.

323. 3 St. grau Kupfer-Erz.

324. 2 St. rothes harigtes Kupfer-Erz.

325. 4 Stangen Japanisches Kupfer (Proben.)

326. 2 Stück Cement-Ihupfer.

327. 2 St. Fahl-Erz.

328. 1 St. dito, christallisirt.

329. 2 St. dito.

350. S St. dito.

351. 3 St. dito.

332. 2 St. Aehrenstein.

333. I St. buntes Kupfer-Erz.

394. 1 grosse Druse Kupferkies, pfauenschweiffarbigt christallisirt auf Kalkspath, aus America.

\$35. 2 St. christallisirtes Thupferlies auf Quarz.

S36. I St. dito dito.

337. 3 St. Kupferkies.

3.38. 4 St. dito.

s39. 4 St. dito.

340. 3 St. dito。

241. 4 St. dito.

342. 5 St. dito.

3ม33. 6 St. dito.

344. St. dito.

345. 1 St. Kupferlasur auf Schweerspath, von Saalfeld.

3ł6. 1 St. Kupferlasur (Coerulium montanum, v. Langenbeck,

347. 1 St. Kupferlasur.

S4s. 3 St. dito.

349. 5 St. diro.

\$50. 3 St. Kupferblak.

\$51. 2 St. dito.

352. 4 St. dito.

353. I angeschlifenc und polirte schöne Malachit-Platte, aus Siberien, gross.

954. 2 Malachite. 
355. 1 Schachtel mit 9 kleinen herzfürmig geschliffenein IMa. lachiten.

356. 3 St. fasrigter Malachit.

35\%. A Stück Malachit, fasrigt.

558. 8 St. dito.

359. 2 St, huprergrün.

360. 2 St. Kupferschiefer.

361. 2 St. Schwefelkies, christallisirt auf Qusrz.

362. 2 St. dito àito.

363. 3 St. dito dito.

364. I St. dito galligt.

365. 1 Schachtel dito diverser.

365. 2 Stück Schwefellies.

367. 3 St. dito.

368. I St. dito.

369. 1 Schachtel dito diverser.

370. \& Stück Leberkies.

371. I St. Magnet-Eisenstein, schön.

372. 1 St. Eisenglanz, christallisirt, von Elba, schön.

373. 1 St. dito, daher, dito.

374. 1 St. dito dito dito.

375. 2 St. dito dito.

376. 2 St. dito, daher, christallisirt, schön.

37\%. 2 St. dito, Fundort unbekannt, nicht christallisirt.".

378. 3 St. dito dito.

379. 3 St. dito dito.

380. 1 St. rother Eisenstein, aus Spanien, pyramidal, schōn.

381. 1 St. dito dito, aus Norberg.

382. 2 St. dito dito (Glaskopf.)

38S. 2 St. dito dito dito.

384. 3 St. dito dito dito.

385. 2 St. dito dito dito.

386. 4 St. dito dito dito.

387. 2 St. brauner dito dito.

389. 3 St. dito dito.

389. 3 St. dito dito.

390. 4 St. dito dito.

391. 2 St. dito dito.

392. 2 St. dito Eisenstein.

393. 1 Schachtel dito dito, diverse.

394. 1 brauner Glaskopf, dendritisch, schön. 
395. 3 Stücl dito dito.

396. 1 Schachtel Dohncin-Erz, Eisensand und Eisenralın.

397. 1 dito mit Sumpf-r.rz.

398. 2 Stück Spath-Eisenstein.

299. 2 St. dito dito.

400. 2 St. dito dito.

401. 2 St. dito dito, schön.

402. 2 St. Bleyglanz, christallisirt, znit Spath-Eisenstein, aus Clausthal.

403. 2 St. dito dito christallisirt, ron St. Maria in Lo. thringen, schön.

404. 2 St. dito dito von Clausthal.

405. 2 St. dito dito dito.

406. 4 St. dito dito diverser, hübsch.

407. I Schachtel dito dito.

408. I Stück Bleyglanz (Sand-Erz) aus Jülich.

409. 2 St. dito.

410. 1 Schachtel diverser dito.

111. 5 Stück verschiedener Bleyglanz.

412. 6 St. dito dito.

413. 4 St. dito dito.

414. 6 St. dito dito.

415. 1 Schachtel dito dito, diverser.

416. 7 Stück Bleyglanz.

417. I Schachtel dito dito.

418. 1 dito dito ditó.

419. I Stück weifser Bleyspath, christallisirt, von Zellerfeld.

420. 1 St. dito ảito, daher.

421. 1 St. dito dito.

422. I St, dito dito mit Kupferblau.

423. 2 St. dito dito dito.

424. 3 St. dito dito dito.

425. 2 St. dito dito dito.

426. 3 St. dito dito dito.

427. 2 St. dito dito mit Kupfergrür.

428. $3 \mathrm{St}$ dito dito dito.

429. Ast. dito dito mit fasrigten Malachic。

430. 1 grüner Bleyspath, aus Breisgau.

431. 1 dito ảito dito.

432. 1 dito dito (schön.)

433. 2 dito dito. 
434. I Stück vitriolsaures Bley (3chön.)

435. I St. dito dito.

436. 1 St. braun Bley-Erz.

437. 2 St. verhättete Bley-Erde.

438. 1 St. Zinnstein, christallisirt, aus England (vorzüglich schön.)

439. I St. dito dito auf Quarz, von Marienberg,

440. 1 St. dito dito aus Böhmen.

441. 2 St. dito dito von Wunsiedel.

442. 4 St. dito dito.i

443. 2 St. dito dito.

444. 3 St. dito dito.

445. 4 St. dito dito.

4x. 2 St. dito dito.

447. I Schachtel diverser dito.

418. 2 Stück Zinnstein (Sachsen.)

449. 4 St. dito.

450. 1 Schachtel dito diverser.

451. 4 Stïck Wolfrahm.

452. 2 St. dito.

453. 3 St. Wismuth.

454. 1 St. braune Blende, aus Siebenbiurgen.

455. 2 St. dito dito.

456. 2 St. dito dito.

457. I Schachtel diverse dito.

458. 2 Stïck rothe Blende in Halkspatln, mit Bleyglanz.

459. 1 St. schwarze dito.

460. 2 St. Galmey.

451. 2 St. graustrahligtes Antinonium (Spiefsglanz.)

462. 1 St. dito dito breitstrahligt.

463. 1 Sto dito dito.

464. 1 St. Quarz mit angeflogenem Antimonium (schön.)

465. 1 St. feinstrahligtes Antimonium, pfauenschweifig gem färbt, angelaufer, auf Quarz (schön.)

466. 1 St. haarigtes Antimonium (Feder-Erz) auf Quarz.

46\%. 1 St. feinstrahligt, wie (No. 465.)

468. I St. grau Braunstein-Erz, christallisirt.

469. 2 Sto dito dito dito strahiigt.

470. 1 St. rothes dito.

471. 1 St. weifser Speis-Kobold, christallisirt,

772. I St. dito dito dito. 
473. 2 St. dito dito vom Harz.

474. 3 St. dito dito, derb, von Andreasberg.

75. 1 St, gestricktes grau Kobold-Erz.

476. 2 St. dito dito.

477. 2 St. dito dito.

478. 1 Schachtel dito diverse.

479. 2 Stïck Kobold-Beschlag.

480. I St. dito.

481. S St. dito.

482. 1 St. Kobold-Blüthe auf Quarz.

489. 2 St. dito dito dito.

484. 4 St. gediegen Arsenik.

485. 2 St. christalisirter Arsenitkies (schön).

486. 1 St. rother rauschgelber dito, von Solfatarra.

487. 1 St. Molubdaen.

488. 2 St. Tunkstein (Scheel-Erz) schön.

489. 1 Schubfach mit Versteinerungen, worunter ein selir grolser Glossopetra (Hayfischzahn.) Trilobiten, Amoniter, Enkriniten, Ostraziten (Hahnlä̈mms.) Eine Wallnuls und eine Kastanie - schön,

490. 1 dito mit Glossopetra, Trilobiten, Amoniten, Ostraziten, Enkriniten, Echiniten. Ein schöıer Orthozeratit.

491. 1 dito Amoniten und Echiniten, diverse.

492. 1 dito mit Echiniten, Ostraziten und Belemniten.

493. 1 dito dito, worunter Hysterolyth, Sandalit, Ostrazit, Griphiten.

494. I Schublade verschiedener rersteinerter Conchylien.

495. 1 dito verschiedener versteinerter Krebse, Knochen und Glossopetrae.

496. 1 Schubfach verschiedener Versteinerungen, mehrentheils Ostraziten.

497. 1 dito verschiedener petrificirter Torallen und Conchylien.

498. 1 dito verschiedener versteinerter Conchylien, meistens Terebratulit und Ostrazit.

499. 1 dito verschiedener Amoniten.

500. 1 dito vereteinerter Conchylien.

501. 1 dito dito, worunter Tubinithen, Orthozeratiten und Entrochiten.

502. 1 dito Amoniten.

503. 1 dito verschiedener, sehr schöncr, versteinerter Con. chylien, als Herz-Muscheln \&x.

504. 1 dito Versteinerungen.

505. 1 Fals mit verschiedenen Mineralien.

506. 1 Schubfach, worin mehrere Smalte Proben.

507. 12 Stüch giöfsere und mehrese hleinere Korallen,

508. 2 St. Ruinen-Marmor in Rahn.

609. 1 Samen-Cabinet. 


\title{
Dritte $\Lambda$ btheilung. \\ Trunstsachen aller Art
}

\author{
von
}

Stein, Elferibein, Holz, Wachs und andren Massen.

1. Ein 2 Fuss loher Obelisk, hübsch geformt, von schön gefürbtem strahlichten Flusspath, aus Derbyshire.

2. 1 kleine Vase, von gleicher Masse.

3 \& 4. 2 kleine Medaillons, die Oberfäche Glas mit Wachs begossen, den unten liegenden Gegenstand zu verdecken.

5. 1 kleine Tasse und Untersatz, von Achat (mocsartig.)

6. 1 ovale Dose, von Serpentin.

7. 1 viereckigte Dose, aus Wismuth-Platten, defect.

8. 1 Pferd anf einem Kubus stehend, aus einem ziemlich grossen Stück Berghristall geschnitzt.

9. 1 liegendes Pferd aus dunklem Serpentin.

10.\} 1 Chinese und Frau, aus Chinesischem Alabasier (Agal. 11. Smatholith.

12. 1 Oberschaale als Biatt, aus Bild - oder Speckstein, in China zierlich geschnitzt.

19. 1 Teller zu Conficturen, desgleichen.

14. 1 Kleine Tasse mit Untersatz, aus Reisstein.

15. Das Portrait eines Ritters, in Schleifstein erhaber ge. schnitten.

16. 1 Triton als Stochknopf, aus einem sehr grossen fast 5 Zoll hohen und breiten Stück Bernstein, wachsgelb, sehr schön geschnitten.

17. 2 kleine Cruzifixe aus Bernstein, sehr zierlich geschnitzt, defect.

18. 1 künstiches Auge von Elfenbein, in einem Futter von Cocusnuss.

19. 1 künstliches Ohr aus Elfenbein.

20. 1 kleines Gestell aus Elfenbein.

21. I von Ludewig von Luike sehr brav aus Elfenbein geschnitzte, circa 2 Fuss hohé Statue Friedrich des 5ten, Königs von Dänemark, kriegerisch gerüstet, den Com. mandostab in der Hand, einherschreitend. Neid und Bosheit mit Füsser tretend. Auf Fussgestell von Miaserholz. Sehr interessantes Stück. 
22. 1 weibliche Figur liegend, zum öffnen, um den Bau der innern Theile des hürpers anatomisch zu zeigen. Sehr brav gearbeitet.

23. 1 mit grosser Kunst wahrscheinlich von Spengler auf der Passigbank gedrechseltes Stück, mit zarter durchbrochener und verschobener Arbeit, in einander gedrechselten Tugein $u$. dergl. Leider beschädigt.

24. 1 schöner Krug von Elfenbein, mit Handhabe und Deckel, mit künstlichen Blumen geziert, im Futter.

25. 1 in A. Dürers Geschmack in Buchsbaumholz trefflich geschnitzte sogenannte Bet-Nuss, zum Oeffnen. Auswenaig gothische Zierathen, inwendig die Geschichte des heiligen Hubertus mit ausnehmend Kleinen Figuren.

26. 1 aus Buchsbaumholz brav geschnitzter Schädel, zum Oeffnen und mit beweglicher Kinnlade.

27. 1 Cruzifix aus Buchsbaum, nicht von grossen Kunstwerth.

28. 1 in Gaislingen künstlich aus Holz gedrechselter Becher, der circa 100 sehr dünne papierdicke Holzbecher in sich fasst."

29. 1 kleine Büchse mit 24 in einander passenden Bechern.

30. 1 kleine Büchse von Cocus, worinn ein Kirschkern, in dem ein Kegelspiel.

31 \& 31 . 1 gedrechselte Schlange und diverse Bruchstücke von Bernstein.

32. 1 hleine Flasche, bernsteinartiger rother Masse.

33. 1 in Ostindien geschnitzter Becher mit Deckel, von eirer grossen Cocusnuss.

34. 1 liegende antique Bronce-Figar.

35. 1 sehr schön gemalter Fächer, von Elfenbein und Perlenmutter, im Futter.

36. I schön abgeformte Wachsbüste, lebensgross.

37. 1 sehr alte kleine Taschenuhr (Nürnbergsches Ey), von frühester Erfindung, ohne Spiral, in einer orientalischen Granatschaale, in Gold gefasst, mit Kette. In Futter.

38. 1 alt Hetrurischer Weinkrug, einfach schwarz, defect.

39. 1 dito Trinkschaale mit Handhabe, defect.

40. 1 hleine Lampe.

41. 1 Pömische Urne nit Ueberresten ron Gebeinen und Asche.

42. 1 desgleichen kleinere, mit Handhaben. 
42a. 1 Opfermesser und 1 Bruchstück desselben.

43. 1 kleine Schaale.

44. 1 ziemlich grosser, circa 14zölliger runder Teller von ächter Majolica. Nayaden und Faunen. Defect, doch sehr gut und wenig bemerkbar zusammengesetzt.

45. 1 dito, ähnlicher Grösse (neuer.)

46. 1 hleiner circa 7 zülliger dito.

47. 1 Becher dito.

48. 1 Schaale von Glas, mit Deckel, mit Inseckten undVer goldnng auf grauem Grunde.

49. 1 Platte mit Blumen. Florentiner Mosaick.

50. 1 dito desgleichen.

51. 1 Römische antique Kupfer-Münze von Otto. Vorseite dessen Brustbild mit Umschrift. Kehrseite, Vereinigung mit Umschrift Securitas P. R.

52. 1 aus Onix geschnittener Mohrenkopf.

53. 1 kleine aus Calceàon geschnittene Figur, karyatidenartig.

54. Sechs kleine diverse geschnittene Steine.

55. 3 St. Bronce-Kupfer, woraus die Statuen Friedrichs des 5 ten und Christian des 6ten gegossen.

56. Ein auf Pergament mit Silberstift sehr schön gezeichnetes Portrait.

57. 1 auf Pergament mit Fractur-Schrift sehx reinlich und schön geschriebenes, mit Zeichnungen mit Tusche geziertes, hleines Gebetbuch, betitelt: Historia vom Leiden und Sterben unsers Herrn Jesu Christi unsers Erlösers.

58. Ein altes Psalterium von Johannes de Turrecremata. Episcop. Sabiniensis, gedr. von Pet. Schöffer.

59. 1 Microscop im Mahagony-Kasten.

60. I Kasten mit einem vorzüglich grossen und 2 kleineren Blasen - und Bezoar - Steinen.

61. 1 grosser Blasen-Stein.

62. 2 Zinndosen, worin Ambra grisea und Balsam moschati.

65. Kleines Modell eines Stuhls zur Selbstfortschaffung an. Füssen Lăhmer.

64. 7 diverse kleine Sauge-Glocken von Glâs.

65. 1 kleine Krone voon Gewürznelken.

66. 1 kleine Koralle aui Fussgestell.

67. 2 Magenbälle von Hornvieh.

68. 1 seltene Fliege im Kasten.

69. 1 dergleichen. 
70. 1 künstlicine Spinne.

71. 7 Schwefelabgüsse von Basrelifs.

72. I Flascheir und zwey Bälle von Federharz, Gummy Ela. sticum.

73. 10 Früchte von Alabaster.

74. 2 Dosen aus Indien, wahrscheinlich mit Gift.

75. Ein Stücł Zeug zum Mantel, aus Bast gewirkt, aus Neu. seeland.

76. 1 desgleichen; aus sehr weissem Zeuge, aus PfanzeirRinde zusanmengesetzt.

77. 1 gleiches.

78. 1 ähnlichəs dünnes, florartig, aus Otaheite.

79. 1 Stück mit wasserdichtem Firniss überzogenen Zenges, von der Insel Amsterdam im Süd-Meer.

80. I St. etwas dünnerem bedruckten Zeuges, daher.

81. 1 St. Zeug, weiss, stark dito, aus Otaheite.

82. 2 hleine gefärbte Zeugstïcke, daher.

83. 1 kleine Matte und ein kleines Ruder aus einem Knochen, aus Neuseeland.

84. 1 Kamm, von der Insel Amsterdam im Süd-Meer.

85. 1 Halsgeschmeide, aus Otaheite.

86. Zu feinen Litzen geflochtenes Haar Otaheitischer Fráuen.

87. I Instrument zum Tatowiren, aus Otaheite.

88. 2 Fischhahmen, von den Bewohnern am Süd-Meer.

89. 1 aus Muscheln zusammengesetztes Instrument, dessen sich die Bewohner des Süd-Meeres beym Schwimmen bedienen.

90. Etwas Bast, aus Neuseeland.

91. 1 Flasche mit Quecksilber.

92. Einige Steine für Mosaik-Arbeit. 


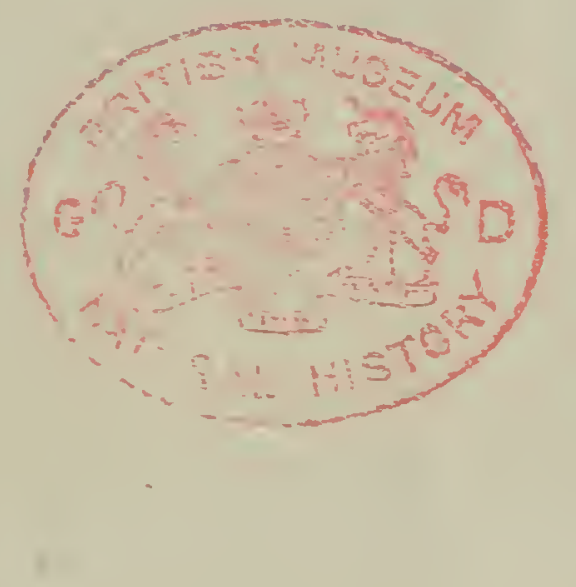




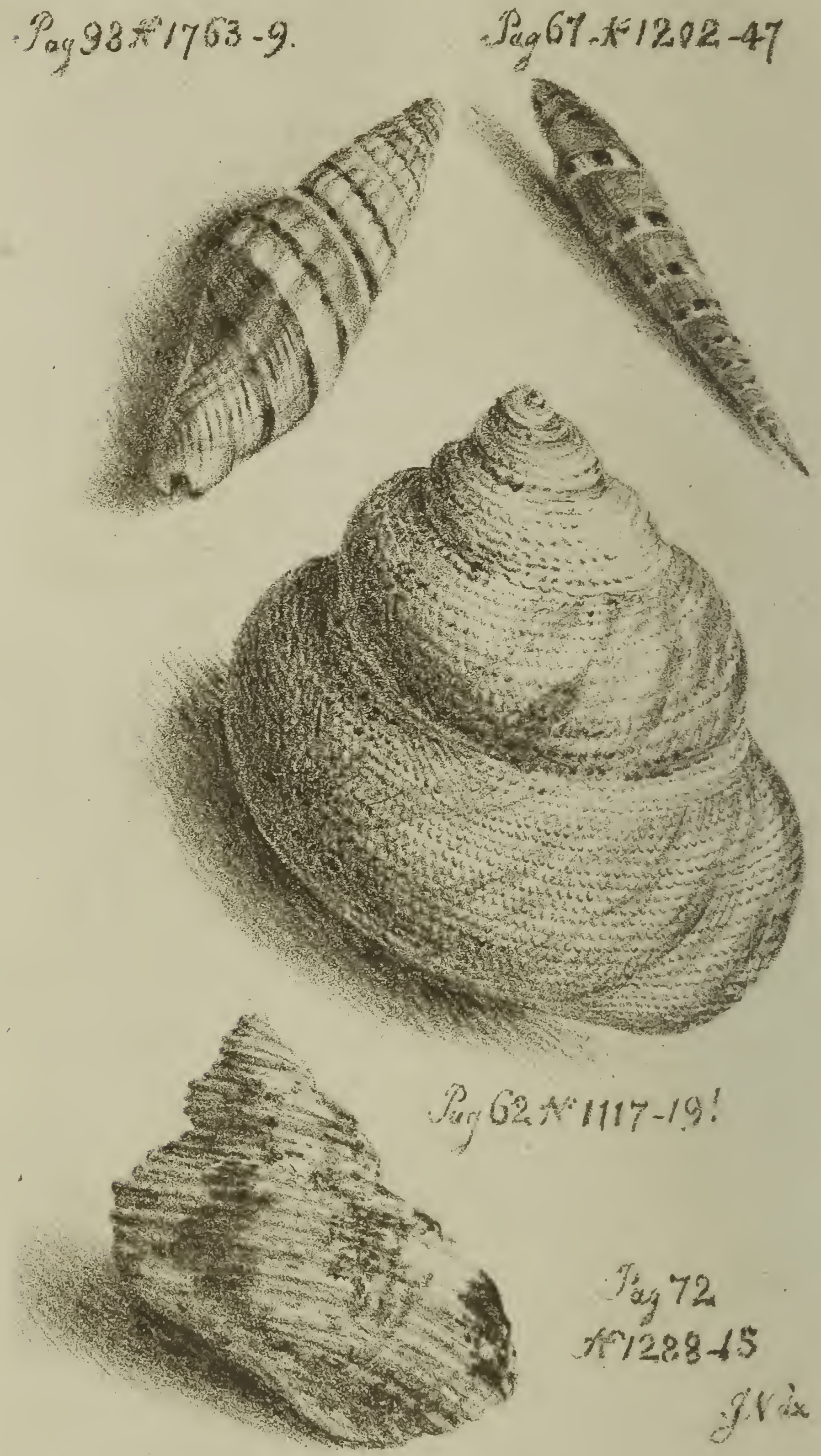




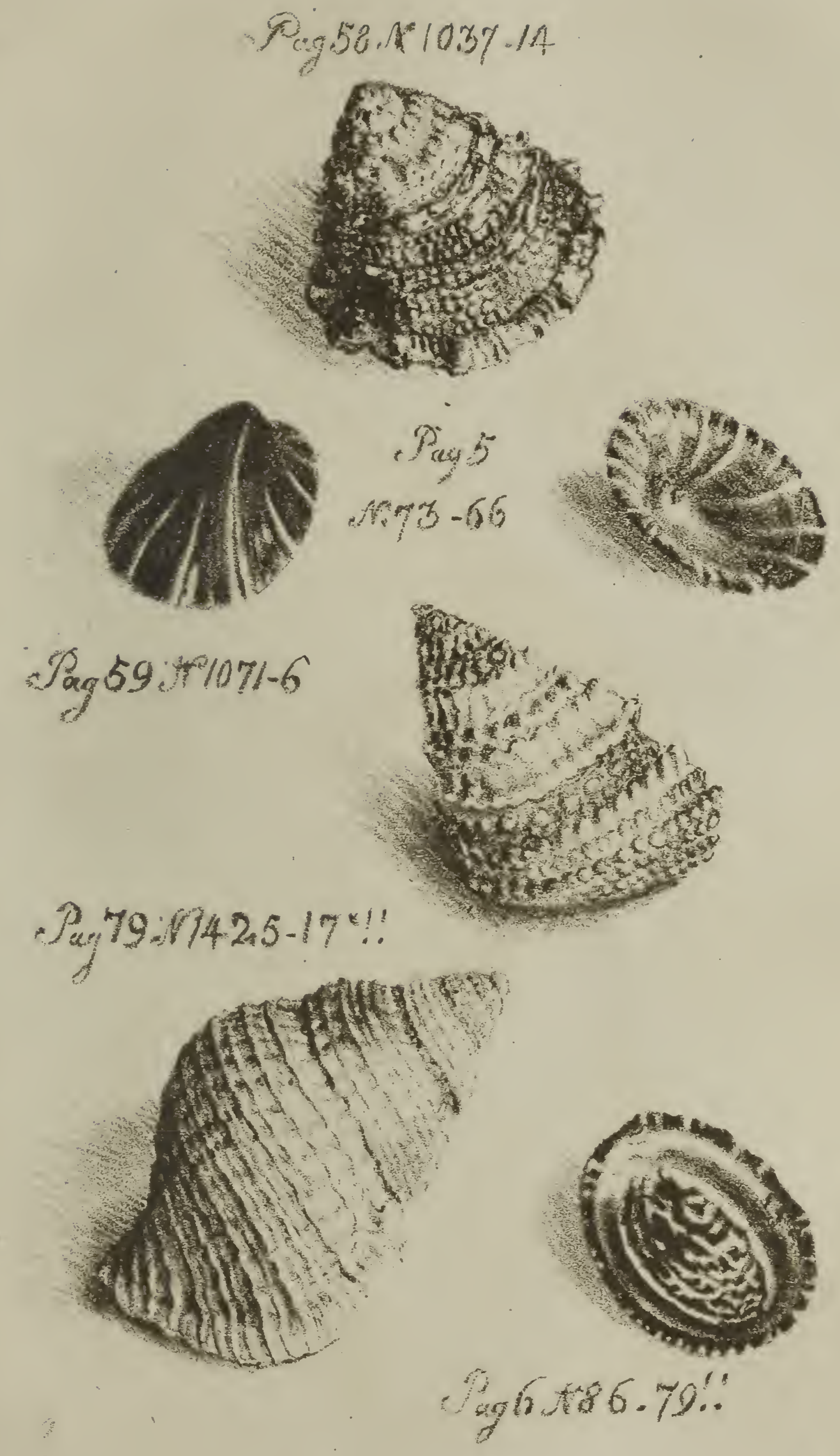




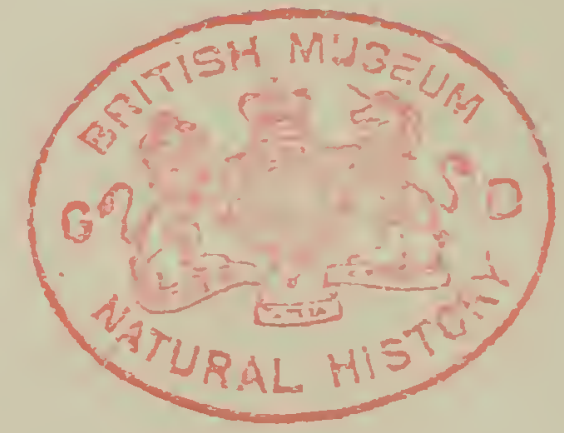




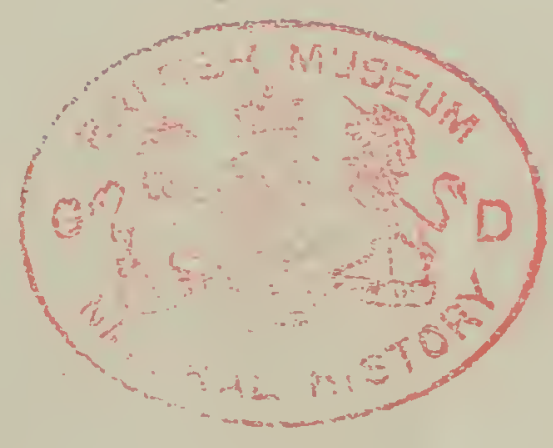




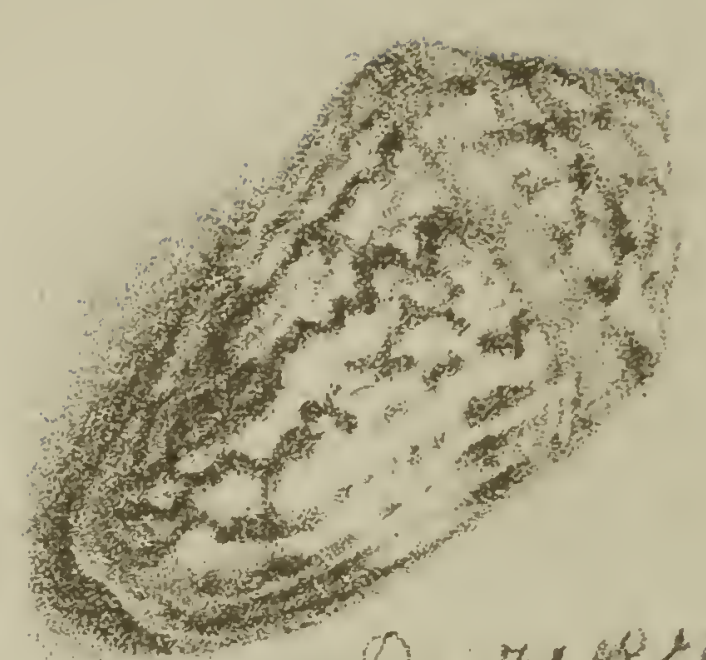

Do.31 1055465 if

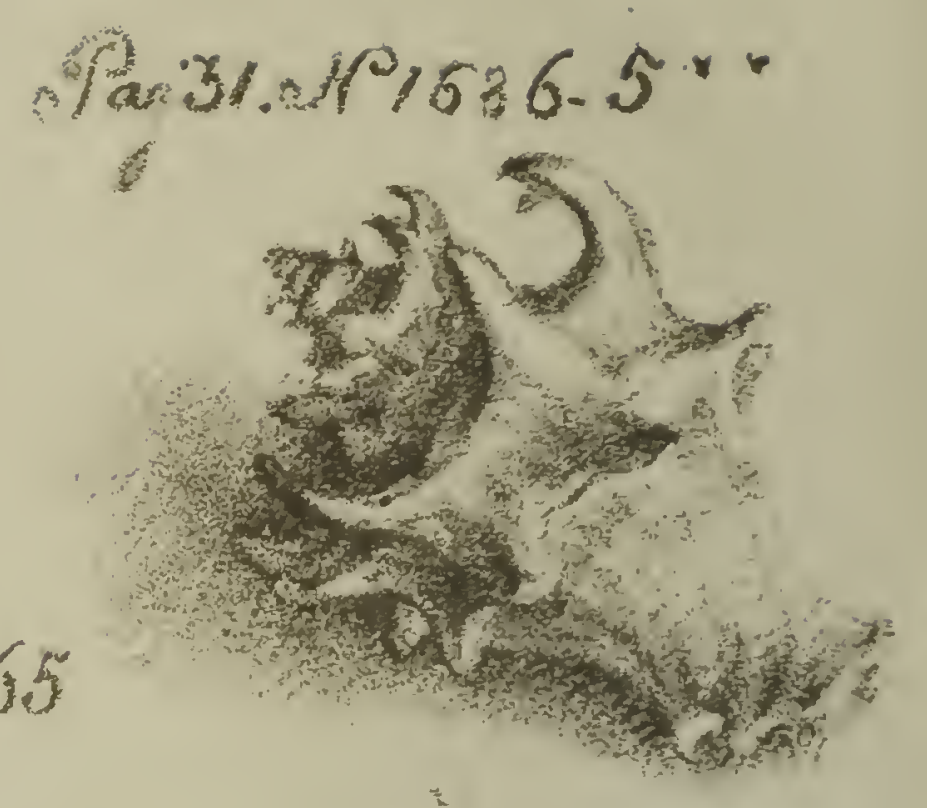

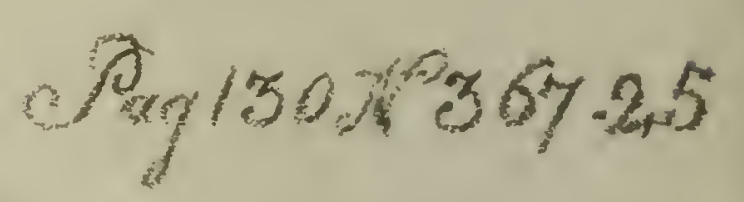

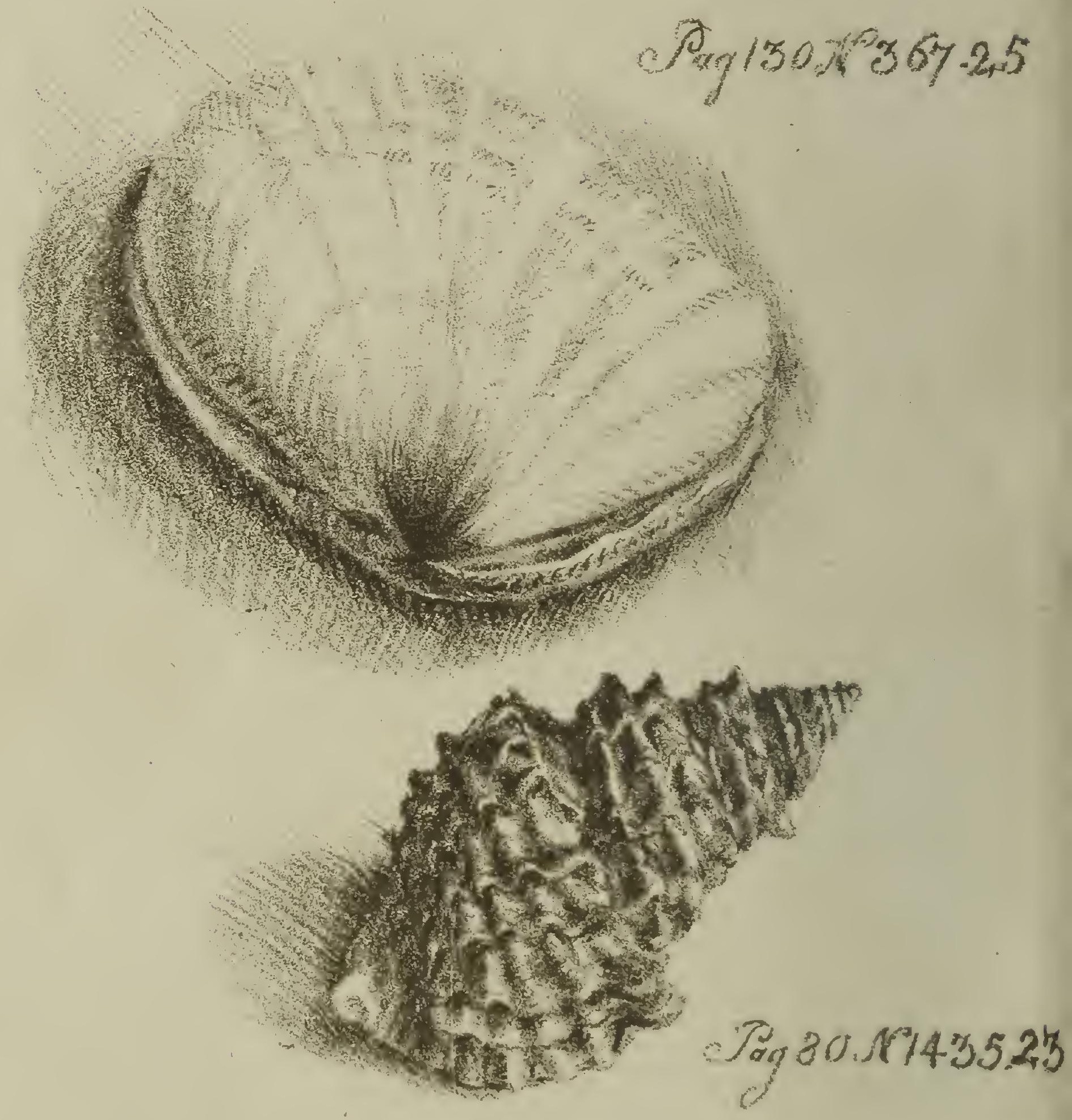

Por?tint 


